\title{
Ir-Catalyzed Regiocontrolled Allylic Amination of Di-/Trienyl Allylic Alcohols with Secondary Amines
}

Shengbiao Tang, † Ziyong Li, † Ying Shao ${ }^{\dagger}$ and Jiangtao Sun*,†

†Jiangsu Key Laboratory of Advanced Catalytic Materials \& Technology, School of Petrochemical Engineering, Changzhou University, 1 Gehu Road, 213164, Changzhou, China

E-mail: jtsun08@gmail.com or jtsun@cczu.edu.cn 


\section{Table of Contents}

1. General information

2. Preparation of substrates and characterization data for new substrates

3. Table S1. Optimization of 3ah and asymmetric synthesis of 4aa'

4. General procedure for sythesis of compounds 3-7

5. Control experiment synthesis of 5ak

6. Preparation for $\mathbf{8}$

7. Preparation for 9

8. References

9. NMR and Chiral HPLC Spectra of compounds 


\section{General information}

All of the reactions were carried out in flame-dried tubes under argon atmosphere. Solvents were dried prior to use. For column chromatography, 200-300 mesh silica gel was used. ${ }^{1} \mathrm{H}$ NMR were recorded on Bruker $300 \mathrm{MHz}, 400 \mathrm{MHz}$ and ${ }^{13} \mathrm{C}$ NMR were recorded on Bruker $75 \mathrm{MHz}, 100 \mathrm{MHz}$ spectrometer in $\mathrm{CDCl}_{3} .{ }^{19} \mathrm{~F}$ NMR were recorded on Bruker $282 \mathrm{MHz}$ spectrometer in $\mathrm{CDCl}_{3}$. Enantiomeric excesses values were determined by HPLC analysis on chiral Daicel Chiralpak OD-H column. Optical rotations were determined on a Rudolph Autopol IV polarimeter. HRMS were performed on Agilent 6540 Q-TOF mass spectrometer (ESI). Melting points were determined on a SGW X-4B melting point apparatus.

\section{Preparation of substrates and characterization data for new substrates}

The secondary amines were all known compounds and prepared according to the literature procedures. ${ }^{[1]}$ The dienyl allylic alcohols were prepared according to the literature procedures. ${ }^{[2]}$

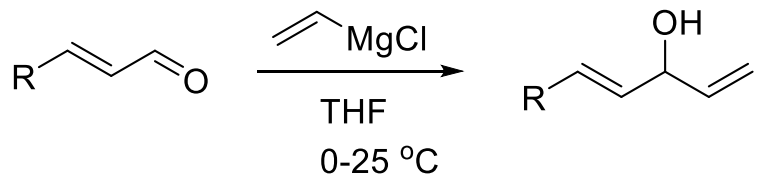

The corresponding aldehyde $(10 \mathrm{mmol}$, lequiv. $)$ was dissolved in THF $(0.5 \mathrm{M})$ and cooled to $0{ }^{\circ} \mathrm{C}$. Vinyl magnesium chlorine (120 mol\%, 1.0 M in THF) was added dropwise, and the reaction was warmed to 25 ${ }^{\circ} \mathrm{C}$ and stirred until the aldehyde was disappeared. The solution was quenched with sat. $\mathrm{NH}_{4} \mathrm{Cl}$ before extracted by ethyl acetate $(3 * 50 \mathrm{~mL})$, and the combined extracts were dried by $\mathrm{Na}_{2} \mathrm{SO}_{4}$. Concentrated in vacuo. The residue was subjected to column chromatography to afford the corresponding dienyl or trienyl allylic alcohols.

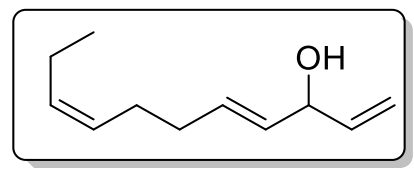

\section{(4E, 8Z)-Undeca-1,4,8-trien-3-ol (1f)}

The title compound was prepared via general procedure, purified by flash chromatography on silica gel eluting with in $\mathrm{EtOAc} / \mathrm{PE}=1 / 10(\mathrm{Rf}=0.35)$, colorless oil, $1.50 \mathrm{~g}, 90 \%$ yield, ${ }^{1} \mathrm{H} \mathrm{NMR}\left(400 \mathrm{MHz}, \mathrm{CDCl}_{3}\right)$ $\delta 5.99-5.82(\mathrm{~m}, 1 \mathrm{H}), 5.71(\mathrm{dt}, J=15.2,6.0 \mathrm{~Hz}, 1 \mathrm{H}), 5.53(\mathrm{dd}, J=15.4,6.8 \mathrm{~Hz}, 1 \mathrm{H}), 5.44-5.29$ (m, 2H), $5.26(\mathrm{~d}, J=17.2 \mathrm{~Hz}, 1 \mathrm{H}), 5.13(\mathrm{~d}, J=10.4 \mathrm{~Hz}, 1 \mathrm{H}), 4.58(\mathrm{t}, J=6.0 \mathrm{~Hz}, 1 \mathrm{H}), 2.18-1.91(\mathrm{~m}, 6 \mathrm{H}), 1.76(\mathrm{br}$, $1 \mathrm{H},-\mathrm{OH}), 0.95(\mathrm{t}, J=7.2 \mathrm{~Hz}, 3 \mathrm{H}) .{ }^{13} \mathrm{C} \mathrm{NMR}\left(100 \mathrm{MHz}, \mathrm{CDCl}_{3}\right) \delta 139.9,132.4(2 \mathrm{C}), 131.4,128.2,114.9$, 73.9, 32.4, 26.8, 20.7, 14.4. HRMS (ESI) calculated for $\mathrm{C}_{11} \mathrm{H}_{19} \mathrm{O}[\mathrm{M}+\mathrm{H}]^{+}$: 167.1436, found: 167.1431 .

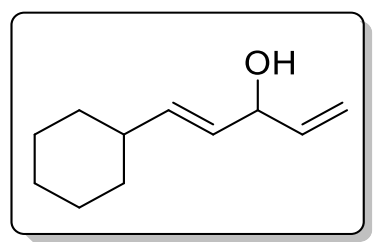


The title compound was prepared via general procedure, purified by flash chromatography on silica gel eluting with in EtOAc/PE $=1 / 10(\mathrm{Rf}=0.41)$, colorless oil, $1.59 \mathrm{~g}, 96 \%$ yield, ${ }^{1} \mathrm{H} \mathrm{NMR}\left(400 \mathrm{MHz}, \mathrm{CDCl}_{3}\right)$ $\delta 5.90(\mathrm{ddd}, J=16.4,10.4,5.6 \mathrm{~Hz}, 1 \mathrm{H}), 5.65$ (dd, $J=15.6,6.4 \mathrm{~Hz}, 1 \mathrm{H}), 5.45$ (dd, $J=15.6,6.8 \mathrm{~Hz}, 1 \mathrm{H})$, $5.24(\mathrm{~d}, J=17.2 \mathrm{~Hz}, 1 \mathrm{H}), 5.11$ (d, $J=10.4 \mathrm{~Hz}, 1 \mathrm{H}), 4.57$ (t, $J=5.7 \mathrm{~Hz}, 1 \mathrm{H}), 1.97-1.90$ (m, 2H), 1.74$1.64(\mathrm{~m}, 5 \mathrm{H}), 1.32-1.03(\mathrm{~m}, 5 \mathrm{H}) .{ }^{13} \mathrm{C} \mathrm{NMR}\left(100 \mathrm{MHz}, \mathrm{CDCl}_{3}\right) \delta 140.1,138.6,128.6,114.7,74.0,40.4$, 32.83, 32.79, 26.2, 26.1(2C). HRMS (ESI) calculated for $\mathrm{C}_{11} \mathrm{H}_{19} \mathrm{O}[\mathrm{M}+\mathrm{H}]^{+}$: 167.1436, found: 167.1434 .

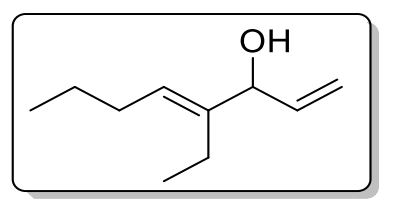

(4E)-4-Ethylocta-1,4-dien-3-ol (1i)

The title compound was prepared via general procedure, purified by flash chromatography on silica gel eluting with in EtOAc/PE $=1 / 10(\mathrm{Rf}=0.52)$, colorless oil, $1.39 \mathrm{~g}, 90 \%$ yield, ${ }^{1} \mathrm{H} \mathrm{NMR}\left(400 \mathrm{MHz}, \mathrm{CDCl}_{3}\right)$ $\delta$ 5.89-5.69 (m, 1H), $5.41(\mathrm{t}, J=7.2 \mathrm{~Hz}, 1 \mathrm{H}), 5.24(\mathrm{dd}, J=17.2,1.6 \mathrm{~Hz}, 1 \mathrm{H}), 5.09(\mathrm{dd}, J=10.4,1.6 \mathrm{~Hz}$, $1 \mathrm{H}), 4.49(\mathrm{~s}, 1 \mathrm{H}), 2.19-1.91(\mathrm{~m}, 5 \mathrm{H}), 1.55-1.20(\mathrm{~m}, 2 \mathrm{H}), 0.97(\mathrm{t}, J=7.6 \mathrm{~Hz}, 3 \mathrm{H}), 0.88(\mathrm{t}, J=7.6 \mathrm{~Hz}, 3 \mathrm{H})$. ${ }^{13} \mathrm{C} \mathrm{NMR}\left(100 \mathrm{MHz}, \mathrm{CDCl}_{3}\right) \delta 142.1,139.9,126.5,114.4,76.8,29.5,22.8,20.4,14.2,13.8$. HRMS (ESI) calculated for $\mathrm{C}_{10} \mathrm{H}_{19} \mathrm{O}[\mathrm{M}+\mathrm{H}]^{+}:$155.1436, found: 155.1433 .

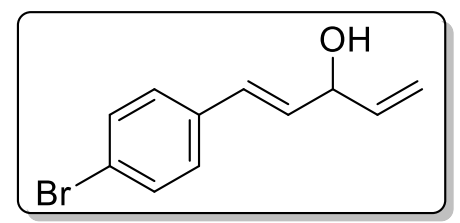

(4E)-1-p-Bromophenylpenta-1,4-dien-3-ol (1m)

The title compound was prepared via general procedure, purified by flash chromatography on silica gel eluting with in $\mathrm{EtOAc} / \mathrm{PE}=1 / 10(\mathrm{Rf}=0.33)$, colorless oil, $2.25 \mathrm{~g}, 95 \%$ yield, ${ }^{1} \mathrm{H} \mathrm{NMR}\left(400 \mathrm{MHz}, \mathrm{CDCl}_{3}\right)$ $\delta 7.44(\mathrm{~d}, J=8.4 \mathrm{~Hz}, 2 \mathrm{H}), 7.25(\mathrm{~d}, J=8.4 \mathrm{~Hz}, 2 \mathrm{H}), 6.56(\mathrm{~d}, J=16.0 \mathrm{~Hz}, 1 \mathrm{H}), 6.24(\mathrm{dd}, J=16.0,6.4 \mathrm{~Hz}$, $1 \mathrm{H}), 6.09-5.80(\mathrm{~m}, 1 \mathrm{H}), 5.35(\mathrm{~d}, J=17.2 \mathrm{~Hz}, 1 \mathrm{H}), 5.22(\mathrm{~d}, J=10.4 \mathrm{~Hz}, 1 \mathrm{H}), 4.81$ (t, $J=6.0 \mathrm{~Hz}, 1 \mathrm{H})$, 2.14 (br, 1H). ${ }^{13} \mathrm{C}$ NMR $\left(100 \mathrm{MHz}, \mathrm{CDCl}_{3}\right) \delta 139.1,135.6,131.8,131.2,129.6,128.2,121.6,115.8,73.8$. HRMS (ESI) calculated for $\mathrm{C}_{11} \mathrm{H}_{12} \mathrm{BrO}[\mathrm{M}+\mathrm{H}]^{+}:$239.0072, found: 239.0064.

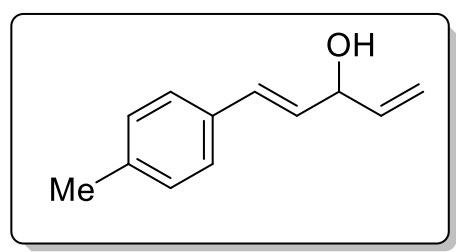

\section{(4E)-1-p-Methylphenylpenta-1,4-dien-3-ol (1n)}

The title compound was prepared via general procedure, purified by flash chromatography on silica gel eluting with in $\mathrm{EtOAc} / \mathrm{PE}=1 / 10(\mathrm{Rf}=0.30)$, colorless oil, $1.71 \mathrm{~g}, 98 \%$ yield, ${ }^{1} \mathrm{H} \mathrm{NMR}\left(400 \mathrm{MHz}, \mathrm{CDCl}_{3}\right)$ $\delta 7.32(\mathrm{~d}, J=8.0 \mathrm{~Hz}, 2 \mathrm{H}), 7.16(\mathrm{~d}, J=8.0 \mathrm{~Hz}, 2 \mathrm{H}), 6.61(\mathrm{~d}, J=16.0 \mathrm{~Hz}, 1 \mathrm{H}), 6.22(\mathrm{dd}, J=16.0,6.4 \mathrm{~Hz}$, $1 \mathrm{H}), 6.13-5.87(\mathrm{~m}, 1 \mathrm{H}), 5.37$ (d, $J=17.2 \mathrm{~Hz}, 1 \mathrm{H}), 5.22(\mathrm{~d}, J=10.4 \mathrm{~Hz}, 1 \mathrm{H}), 4.92-4.56(\mathrm{~m}, 1 \mathrm{H}), 2.38(\mathrm{~s}$, 
$\left.4 \mathrm{H},-\mathrm{CH}_{3},-\mathrm{OH}\right) .{ }^{13} \mathrm{C} \mathrm{NMR}\left(100 \mathrm{MHz}, \mathrm{CDCl}_{3}\right) \delta 139.5,137.7,133.9,130.8,129.4,129.3,126.5,115.3$, 73.9, 21.3. HRMS (ESI) calculated for $\mathrm{C}_{12} \mathrm{H}_{15} \mathrm{O}[\mathrm{M}+\mathrm{H}]^{+}: 175.1123$, found: 175.1118 .

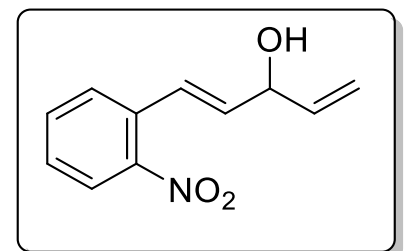

(4E)-1-o-nitrophenylpenta-1,4-dien-3-ol (1q)

The title compound was prepared via general procedure, purified by flash chromatography on silica gel eluting with in EtOAc/PE $=1 / 3(\mathrm{Rf}=0.40)$, brown oil, $1.27 \mathrm{~g}, 62 \%$ yield, ${ }^{1} \mathrm{H} \mathrm{NMR}\left(400 \mathrm{MHz}, \mathrm{CDCl}_{3}\right) \delta$ $7.94(\mathrm{~d}, J=8.0 \mathrm{~Hz}, 1 \mathrm{H}), 7.70-7.51(\mathrm{~m}, 2 \mathrm{H}), 7.45-7.31(\mathrm{~m}, 1 \mathrm{H}), 7.11(\mathrm{~d}, J=15.6 \mathrm{~Hz}, 1 \mathrm{H}), 6.22(\mathrm{dd}, J=$ 15.6, 6.0 Hz, 1H), 6.08-5.84 (m, 1H), $5.38(\mathrm{~d}, J=17.2 \mathrm{~Hz}, 1 \mathrm{H}), 5.24(\mathrm{~d}, J=10.4 \mathrm{~Hz}, 1 \mathrm{H}), 4.88(\mathrm{~m}, 1 \mathrm{H})$, $1.85(\mathrm{~d}, J=4.0 \mathrm{~Hz}, 1 \mathrm{H},-\mathrm{OH}) .{ }^{13} \mathrm{C} \mathrm{NMR}\left(100 \mathrm{MHz}, \mathrm{CDCl}_{3}\right) \delta 147.9,138.6,135.7,133.1,132.4,128.8$, 128.3, 126.0, 124.6, 116.1, 73.5. HRMS (ESI) calculated for $\mathrm{C}_{11} \mathrm{H}_{12} \mathrm{NO}_{3}[\mathrm{M}+\mathrm{H}]^{+}:$206.0817, found: 206.0822 .

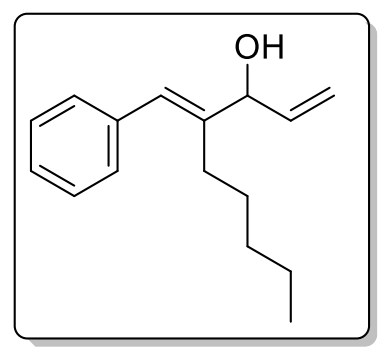

(4E)-1-phenyl-2-pentyl-penta-1,4-dien-3-ol (1r)

The title compound was prepared via general procedure, purified by flash chromatography on silica gel eluting with in EtOAc/PE $=1 / 10(\mathrm{Rf}=0.38)$, colorless oil, $2.01 \mathrm{~g}, 88 \%$ yield, ${ }^{1} \mathrm{H} \mathrm{NMR}\left(400 \mathrm{MHz}, \mathrm{CDCl}_{3}\right)$ $\delta$ 7.41-7.22 (m, 5H), 6.64 (s, 1H), 5.97 (ddd, $J=16.4,10.4,6.0 \mathrm{~Hz}, 1 \mathrm{H}), 5.41(\mathrm{~d}, J=17.2 \mathrm{~Hz}, 1 \mathrm{H}), 5.26$ $(\mathrm{d}, J=10.4 \mathrm{~Hz}, 1 \mathrm{H}), 4.75(\mathrm{~d}, J=6.0 \mathrm{~Hz}, 1 \mathrm{H}), 2.39$ (dt, $J=13.6,8.0 \mathrm{~Hz}, 1 \mathrm{H}), 2.21$ (dt, $J=13.6,8.0 \mathrm{~Hz}$, $1 \mathrm{H}), 2.00(\mathrm{~s}, 1 \mathrm{H}), 1.63-1.42(\mathrm{~m}, 2 \mathrm{H}), 1.38-1.24(\mathrm{~m}, 4 \mathrm{H}), 0.91(\mathrm{t}, J=6.8 \mathrm{~Hz}, 3 \mathrm{H}) .{ }^{13} \mathrm{C}$ NMR $(100 \mathrm{MHz}$, $\left.\mathrm{CDCl}_{3}\right) \delta 143.9,139.5,137.7,128.7,128.2,126.6,125.9,115.7,76.9,32.2,28.7,28.6,22.4,14.1$. HRMS (ESI) calculated for $\mathrm{C}_{16} \mathrm{H}_{23} \mathrm{O}[\mathrm{M}+\mathrm{H}]^{+}: 231.1749$, found: 231.1745 .

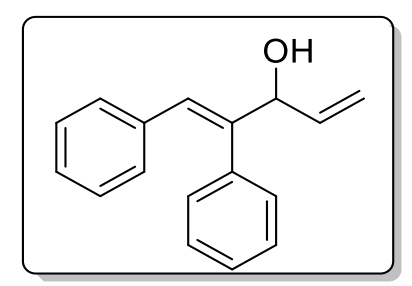

(4E)-1,2-Diphenylpenta-1,4-dien-3-ol (1t)

The title compound was prepared via general procedure, purified by flash chromatography on silica gel eluting with in EtOAc/PE = 1/5 $(\mathrm{Rf}=0.38)$, yellow oil, $1.94 \mathrm{~g}, 83 \%$ yield, ${ }^{1} \mathrm{H}$ NMR $\left(300 \mathrm{MHz}, \mathrm{CDCl}_{3}\right)$ $\delta$ 7.36-7.23 (m, 3H), 7.22-7.15 (m, 2H), 7.10-7.03 (m, 3H), 6.97-6.92 (m, 2H), 6.71 (s, 1H), 5.93 (ddd, $J$ 
$=17.1,10.2,6.0 \mathrm{~Hz}, 1 \mathrm{H}), 5.25(\mathrm{dt}, J=17.1,1.5 \mathrm{~Hz}, 1 \mathrm{H}), 5.15(\mathrm{dt}, J=10.5,1.5 \mathrm{~Hz}, 1 \mathrm{H}), 4.95(\mathrm{dd}, J=$ 6.0, $0.9 \mathrm{~Hz}, 1 \mathrm{H}), 2.06$ (br, 1H). ${ }^{13} \mathrm{C} \mathrm{NMR}\left(75 \mathrm{MHz}, \mathrm{CDCl}_{3}\right) \delta 143.2,138.7,138.2,136.4,129.4,129.3$, 128.7, 127.9, 127.5, 127.3, 126.9, 115.9, 78.0. HRMS (ESI) calculated for $\mathrm{C}_{17} \mathrm{H}_{17} \mathrm{O}[\mathrm{M}+\mathrm{H}]^{+}: 237.1279$, found: 237.1280 .

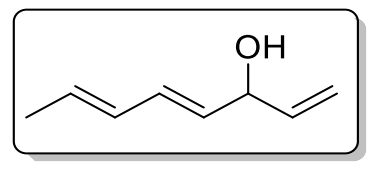

\section{(4E,6E)-Octa-1,4,6-trien-3-ol (1a')}

The title compound was prepared via general procedure, purified by flash chromatography on silica gel eluting with in EtOAc/PE $=1 / 10(\mathrm{Rf}=0.41)$, yellow oil, $1.13 \mathrm{~g}, 92 \%$ yield, ${ }^{1} \mathrm{H} \mathrm{NMR}\left(400 \mathrm{MHz}, \mathrm{CDCl}_{3}\right)$ $\delta 6.20(\mathrm{dd}, J=15.2,10.4 \mathrm{~Hz}, 1 \mathrm{H}), 6.05$ (ddd, $J=14.8,10.4,1.6 \mathrm{~Hz}, 1 \mathrm{H}), 5.89$ (ddd, $J=17.4,10.4,6.0$ $\mathrm{Hz}, 1 \mathrm{H}), 5.72(\mathrm{dq}, J=13.6,6.8 \mathrm{~Hz}, 1 \mathrm{H}), 5.57(\mathrm{dd}, J=15.2,6.8 \mathrm{~Hz}, 1 \mathrm{H}), 5.25(\mathrm{dt}, J=17.2,1.2 \mathrm{~Hz}, 1 \mathrm{H})$, $5.12(\mathrm{dt}, J=10.4,1.2 \mathrm{~Hz}, 1 \mathrm{H}), 4.63(\mathrm{t}, J=6.2 \mathrm{~Hz}, 1 \mathrm{H}), 2.09(\mathrm{br}, 1 \mathrm{H}), 1.76(\mathrm{dd}, J=6.8,1.2 \mathrm{~Hz}, 3 \mathrm{H}) .{ }^{13} \mathrm{C}$ NMR (100 MHz, $\left.\mathrm{CDCl}_{3}\right) \delta 139.5,131.4,131.2,130.8,130.5,115.1,73.6,18.2$. HRMS (ESI) calculated for $\mathrm{C}_{8} \mathrm{H}_{13} \mathrm{O}[\mathrm{M}+\mathrm{H}]^{+}:$125.0966, found: 125.0965 .

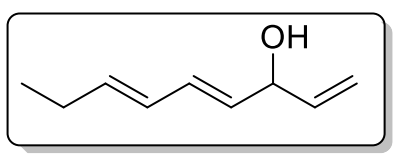

\section{(4E,6E)-Nona-1,4,6-trien-3-ol (1b')}

The title compound was prepared via general procedure, purified by flash chromatography on silica gel eluting with in EtOAc/PE $=1 / 10(\mathrm{Rf}=0.28)$, yellow oil, $1.30 \mathrm{~g}, 94 \%$ yield, ${ }^{1} \mathrm{H} \mathrm{NMR}\left(400 \mathrm{MHz}, \mathrm{CDCl}_{3}\right)$ $\delta 6.21(\mathrm{dd}, J=15.2,10.4 \mathrm{~Hz}, 1 \mathrm{H}), 6.03$ (dd, $J=15.2,10.4 \mathrm{~Hz}, 1 \mathrm{H}), 5.89$ (ddd, $J=17.2,10.4,5.6 \mathrm{~Hz}$, $1 \mathrm{H}), 5.81-5.68(\mathrm{~m}, 1 \mathrm{H}), 5.60(\mathrm{dd}, J=15.2,6.8 \mathrm{~Hz}, 1 \mathrm{H}), 5.26(\mathrm{dt}, J=17.2,1.2 \mathrm{~Hz}, 1 \mathrm{H}), 5.13(\mathrm{dt}, J=10.4$, $1.2 \mathrm{~Hz}, 1 \mathrm{H}), 4.64(\mathrm{t}, J=6.0 \mathrm{~Hz}, 1 \mathrm{H}), 2.11(\mathrm{p}, J=6.8 \mathrm{~Hz}, 2 \mathrm{H}), 1.97(\mathrm{br}, 1 \mathrm{H}), 1.01(\mathrm{t}, J=7.5 \mathrm{~Hz}, 3 \mathrm{H}) .{ }^{13} \mathrm{C}$ NMR $\left(100 \mathrm{MHz}, \mathrm{CDCl}_{3}\right) \delta 139.5,137.6,131.6,131.4,128.5,115.1,73.7,25.7,13.5$. HRMS (ESI) calculated for $\mathrm{C}_{9} \mathrm{H}_{15} \mathrm{O}[\mathrm{M}+\mathrm{H}]^{+}:$139.1123, found: 139.1125 .

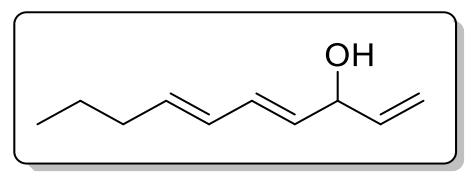

\section{(4E,6E)-Deca-1,4,6-trien-3-ol (1c')}

The title compound was prepared via general procedure, purified by flash chromatography on silica gel eluting with in EtOAc/PE = 1/10 ( Rf=0.36), Yellow oil, $1.40 \mathrm{~g}, 92 \%$ yield, ${ }^{1} \mathrm{H}$ NMR $\left(400 \mathrm{MHz}, \mathrm{CDCl}_{3}\right)$ $\delta 6.21(\mathrm{dd}, J=15.2,10.4 \mathrm{~Hz}, 1 \mathrm{H}), 6.03(\mathrm{dd}, J=15.2,10.4 \mathrm{~Hz}, 1 \mathrm{H}), 5.95-5.82(\mathrm{~m}, 1 \mathrm{H}), 5.78-5.65(\mathrm{~m}, 1 \mathrm{H})$, $5.59(\mathrm{dd}, J=15.2,6.8 \mathrm{~Hz}, 1 \mathrm{H}), 5.26(\mathrm{~d}, J=17.2 \mathrm{~Hz}, 1 \mathrm{H}), 5.13(\mathrm{~d}, J=10.4 \mathrm{~Hz}, 1 \mathrm{H}), 4.63$ (t, $J=6.0 \mathrm{~Hz}$, $1 \mathrm{H}), 2.12-2.03\left(\mathrm{~m}, 3 \mathrm{H},-\mathrm{CH}_{2}-,-\mathrm{OH}\right), 1.48-1.33(\mathrm{~m}, 2 \mathrm{H}), 0.90(\mathrm{t}, J=7.2 \mathrm{~Hz}, 3 \mathrm{H}) .{ }^{13} \mathrm{C}$ NMR $(100 \mathrm{MHz}$, $\left.\mathrm{CDCl}_{3}\right) \delta 139.5,135.9,131.6,131.4,129.6,115.1,73.7,34.8,22.4,13.7$. HRMS (ESI) calculated for 
$\mathrm{C}_{10} \mathrm{H}_{17} \mathrm{O}[\mathrm{M}+\mathrm{H}]^{+}:$153.1279, found: 153.1276 .

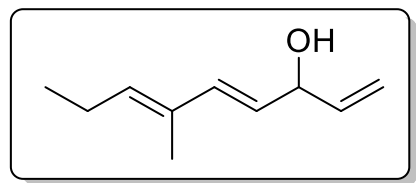

\section{(4E,6E)-Deca-1,4,6-trien-3-ol (1d')}

The title compound was prepared via general procedure, purified by flash chromatography on silica gel eluting with in EtOAc/PE $=1 / 10(\mathrm{Rf}=0.40)$, yellow oil, $1.38 \mathrm{~g}, 91 \%$ yield, ${ }^{1} \mathrm{H} \mathrm{NMR}\left(300 \mathrm{MHz}, \mathrm{CDCl}_{3}\right)$ $\delta 6.26(\mathrm{~d}, J=15.6 \mathrm{~Hz}, 1 \mathrm{H}), 5.93(\mathrm{ddd}, J=17.1,10.2,6.0 \mathrm{~Hz}, 1 \mathrm{H}), 5.71-5.45(\mathrm{~m}, 2 \mathrm{H}), 5.28(\mathrm{dt}, J=17.1$, $1.5 \mathrm{~Hz}, 1 \mathrm{H}), 5.14(\mathrm{dt}, J=10.2,1.5 \mathrm{~Hz}, 1 \mathrm{H}), 4.68(\mathrm{t}, J=6.0 \mathrm{~Hz}, 1 \mathrm{H}), 2.14(\mathrm{p}, J=7.5 \mathrm{~Hz}, 2 \mathrm{H}), 1.74(\mathrm{~s}$, 3H), 1.65 (br, 1H, -OH), 0.99 (t, $J=7.5 \mathrm{~Hz}, 3 \mathrm{H}) .{ }^{13} \mathrm{C} \mathrm{NMR}\left(75 \mathrm{MHz}, \mathrm{CDCl}_{3}\right) \delta 139.8,136.5,135.9,132.4$, 127.2, 115.0, 74.3, 21.7, 14.1, 12.4. HRMS (ESI) calculated for $\mathrm{C}_{10} \mathrm{H}_{17} \mathrm{O}[\mathrm{M}+\mathrm{H}]^{+}:$153.1279, found: 153.1276.

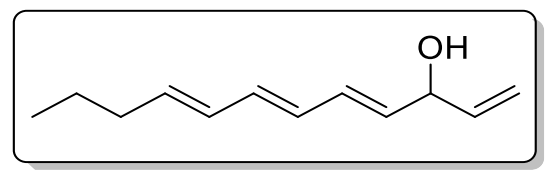

\section{$(4 E, 6 E, 8 E)$-dodeca-1,4,6,8-tetraen-3-ol (1e')}

The title compound was prepared via general procedure, purified by flash chromatography on silica gel eluting with in EtOAc/PE = 1/10 ( Rf = 0.25), yellow oil, 1.45g, 81\% yield, ${ }^{1} \mathrm{H}$ NMR $\left(400 \mathrm{MHz}, \mathrm{CDCl}_{3}\right)$ $\delta$ 6.31-6.18 (m, 2H), 6.16-6.02 (m, 2H), $5.90(\mathrm{ddd}, J=17.2,10.4,6.0 \mathrm{~Hz}, 1 \mathrm{H}), 5.77-5.65(\mathrm{~m}, 2 \mathrm{H}), 5.27$ $(\mathrm{dt}, J=17.2,1.2 \mathrm{~Hz}, 1 \mathrm{H}), 5.15(\mathrm{dt}, J=10.4,1.2 \mathrm{~Hz}, 1 \mathrm{H}), 4.67(\mathrm{t}, J=6.0 \mathrm{~Hz}, 1 \mathrm{H}), 2.13-2.04(\mathrm{~m}, 3 \mathrm{H}, \mathrm{OH}$, $\left.-\mathrm{CH}_{2}-\right), 1.49-1.34(\mathrm{~m}, 2 \mathrm{H}), 0.92(\mathrm{t}, J=7.2 \mathrm{~Hz}, 3 \mathrm{H}) .{ }^{13} \mathrm{C} \mathrm{NMR}\left(100 \mathrm{MHz}, \mathrm{CDCl}_{3}\right) \delta 139.4,136.1,134.1$, 133.0, 131.5, 130.4, 129.5, 115.2, 73.7, 35.0, 22.5, 13.8. HRMS (ESI) calculated for $\mathrm{C}_{12} \mathrm{H}_{19} \mathrm{O}[\mathrm{M}+\mathrm{H}]^{+}$: 179.1436, found: 179.1433 .

\section{Table S1 Optimization of 3aj and asymmetric synthesis of 4aj ${ }^{a}$}

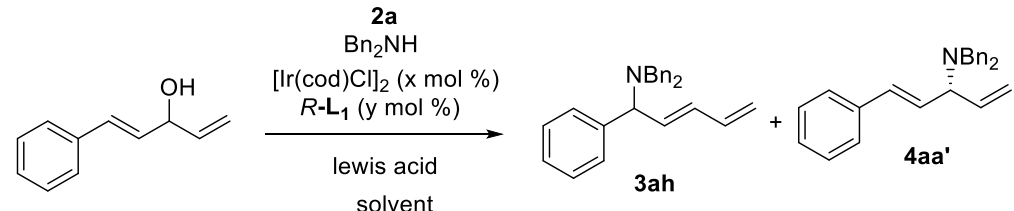

$1 \mathrm{j}$

solvent
rt. $24 \mathrm{~h}$

$0.1 \mathrm{mmol}$

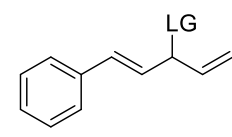

$1 ", R=O A C$

$1 " ', R=O B o c$

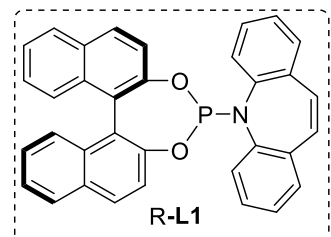

\begin{tabular}{llllllll}
\hline entry & solvent & $\mathrm{x} / \mathrm{y}$ & $\mathbf{1 / 2}$ & additive & ${\text { yield }(\%)^{b}}^{b}$ & ratio: $3 / 4^{c}$ & ee (4) $)^{d}$ \\
\hline 1 & DCM & $1 / 4$ & $1 / 2.5$ & $\mathrm{Fe}(\mathrm{OTf})_{2}$ & 63 & $2.5 / 1$ & $86 \%$ \\
2 & DCM & $1 / 4$ & $1 / 2.5$ & $\mathrm{Zn}(\mathrm{OTf})_{2}$ & 66 & $3.1 / 1$ & N.D. \\
3 & DCM & $1 / 4$ & $1 / 2.5$ & $\operatorname{In}(\mathrm{OTf})_{3}$ & 27 & N.D. & N.D
\end{tabular}




\begin{tabular}{|c|c|c|c|c|c|c|c|}
\hline 4 & DCM & $1 / 4$ & $1 / 2.5$ & $\mathrm{Sc}(\mathrm{OTf})_{3}$ & 72 & $4.9 / 1$ & N.D \\
\hline 5 & DCM & $1 / 4$ & $1 / 2.5$ & $\mathrm{Yb}(\mathrm{OTf})_{3}$ & 68 & $4.2 / 1$ & N.D \\
\hline 6 & DCM & $1 / 4$ & $1 / 2.5$ & $\mathrm{Yt}(\mathrm{OTf})_{3}$ & 56 & $3 / 1$ & N.D \\
\hline 7 & DCM & $1 / 4$ & $1 / 4$ & $\mathrm{Sc}(\mathrm{OTf})_{3}$ & 79 & $5.1 / 1$ & N.D. \\
\hline 8 & DCM & $1 / 4$ & $1 / 4$ & TFA & 26 & - & N.D. \\
\hline 9 & DCE & $1 / 4$ & $1 / 4$ & $\mathrm{Sc}(\mathrm{OTf})_{3}$ & 80 & $3.6 / 1$ & N.D. \\
\hline 10 & $\mathrm{CHCl}_{3}$ & $1 / 4$ & $1 / 4$ & $\mathrm{Sc}(\mathrm{OTf})_{3}$ & 64 & $5 / 1$ & N.D. \\
\hline 11 & $\mathrm{CCl}_{4}$ & $1 / 4$ & $1 / 4$ & $\mathrm{Sc}(\mathrm{OTf})_{3}$ & 42 & $1 / 3$ & N.D. \\
\hline 12 & THF & $1 / 4$ & $1 / 4$ & $\mathrm{Sc}(\mathrm{OTf})_{3}$ & trace & - & N.D. \\
\hline 13 & $\begin{array}{l}1,4- \\
\text { dioxane }\end{array}$ & $1 / 4$ & $1 / 4$ & $\mathrm{Sc}(\mathrm{OTf})_{3}$ & - & - & N.D. \\
\hline 14 & toluene & $1 / 4$ & $1 / 4$ & $\mathrm{Sc}(\mathrm{OTf})_{3}$ & 78 & $1 / 10$ & $99 \%$ \\
\hline 15 & hexane & $1 / 4$ & $1 / 4$ & $\mathrm{Sc}(\mathrm{OTf})_{3}$ & trace & - & N.D. \\
\hline 16 & $\mathrm{Et}_{2} \mathrm{O}$ & $1 / 4$ & $1 / 4$ & $\mathrm{Sc}(\mathrm{OTf})_{3}$ & 46 & $4.1 / 1$ & N.D. \\
\hline 17 & $\mathrm{CH}_{3} \mathrm{CN}$ & $1 / 4$ & $1 / 4$ & $\mathrm{Sc}(\mathrm{OTf})_{3}$ & 60 & $1 / 5$ & $78 \%$ \\
\hline 18 & EtOH & $1 / 4$ & $1 / 4$ & $\mathrm{Sc}(\mathrm{OTf})_{3}$ & 32 & N.D & N.D. \\
\hline 19 & DMF & $1 / 4$ & $1 / 4$ & $\mathrm{Sc}(\mathrm{OTf})_{3}$ & - & - & N.D. \\
\hline 20 & DCM & $1 / 3$ & $1 / 4$ & $\mathrm{Sc}(\mathrm{OTf})_{3}$ & 76 & $3.2 / 1$ & N.D. \\
\hline 21 & DCM & $1 / 2$ & $1 / 4$ & $\mathrm{Sc}(\mathrm{OTf})_{3}$ & 46 & $2.2 / 1$ & N.D. \\
\hline 22 & DCM & $1 / 4$ & $2 / 1$ & $\mathrm{Sc}(\mathrm{OTf})_{3}$ & trace & N.D. & N.D. \\
\hline 23 & DCM & $1 / 4$ & $1 / 1$ & $\mathrm{Sc}(\mathrm{OTf})_{3}$ & trace & N.D. & N.D. \\
\hline 24 & DCM & $1 / 4$ & $1 / 2$ & $\mathrm{Sc}(\mathrm{OTf})_{3}$ & 73 & $5 / 1$ & N.D. \\
\hline 25 & DCM & $1 / 4$ & $1 / 8$ & $\mathrm{Sc}(\mathrm{OTf})_{3}$ & 45 & N.D. & N.D. \\
\hline 26 & $\begin{array}{l}\text { 1" instead } \\
\text { entry } 4\end{array}$ & of 1 & in the & condition of & 91 & $2.5 / 1$ & $95 \%$ \\
\hline 27 & $\begin{array}{l}1 " \text { instead } \\
\text { entry } 4\end{array}$ & of 1 & in the & condition of & 76 & $3.5 / 1$ & $92 \%$ \\
\hline
\end{tabular}

${ }^{a}$ General conditions: $1(0.2 \mathrm{mmol}), \mathbf{2},[\operatorname{Ir}(\operatorname{cod}) \mathrm{Cl}]_{2}(\mathrm{x}$ mol \%), $(S)-\mathbf{L 1}(\mathrm{y} \%)$, and additive in solvent $(2 \mathrm{~mL})$ at room temperature for $48 \mathrm{~h}$. ${ }^{b}$ Yield of isolated product. ${ }^{c}$ Determined by ${ }^{1} \mathrm{H}$ NMR analysis. ${ }^{d}$ Determined by HPLC analysis. N.D.: not detected.

\section{General procedure for the synthesis and experiment data of 3-7.}

a)

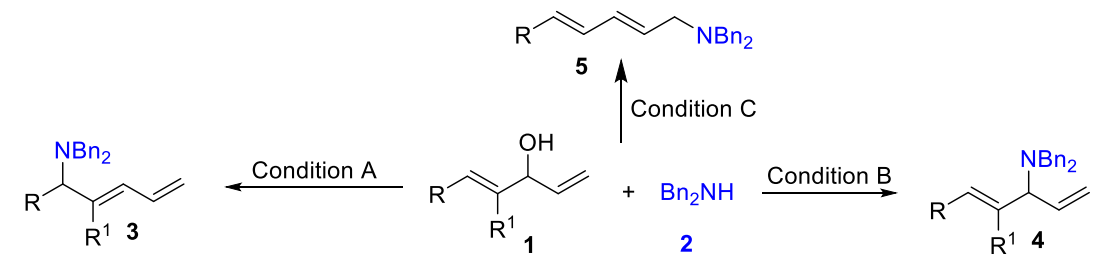

b)

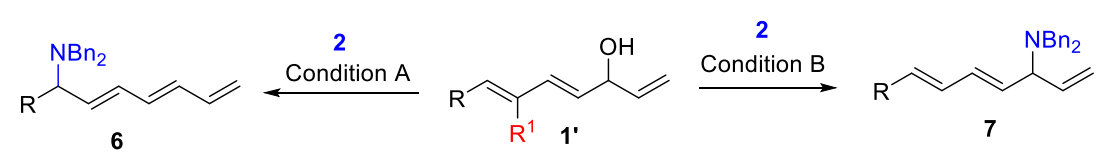

General procedure A: a flame dried tube was cooled to rt. and charged with $[\operatorname{Ir}(\operatorname{cod}) \mathrm{Cl}]_{2}(5.2 \mathrm{mg}, 8$ $\mu \mathrm{mol}, 4 \mathrm{~mol} \%$ ) and ligand $\mathbf{L 1}(16.0 \mathrm{mg}, 32 \mu \mathrm{mol}, 16 \mathrm{~mol} \%)$. The tube backfilled with $\mathrm{N}_{2}$. The flask was added freshly distilled DCM $(2.0 \mathrm{~mL})$, and stirred for $15 \mathrm{~min}$. Then the allyl alcohols $1(0.2 \mathrm{mmol}, 1.0$ equiv), secondary amines 2 (0.8 mmol, 4.0 equiv.), and $\mathrm{Sc}(\mathrm{OTf})_{3}(98 \mathrm{mg}, 0.2 \mathrm{mmol}, 100 \mathrm{~mol} \%$ ) were added. The tube was sealed and stirred at rt. After the reaction was complete (monitored by TLC), the solution was quenched with water $(10 \mathrm{~mL})$ and extracted with DCM $(3 \times 10 \mathrm{~mL})$. The organic layers dried 
over $\mathrm{Na}_{2} \mathrm{SO}_{4}$ and concentrated by rotary evaporation. The crude product was purified by silica gel column chromatography (DCM/PE $=1 / 5-1 / 10)$ to afford the desired products (3aa-3aq, 3ba-3ha, 6aa-6ah).

General procedure B: a flame dried tube was cooled to $\mathrm{rt}$. and charged with $[\operatorname{Ir}(\operatorname{cod}) \mathrm{Cl}]_{2}(5.2 \mathrm{mg}, 8$ $\mu \mathrm{mol}, 4 \mathrm{~mol} \%$ ) and ligand $\mathbf{L 1}\left(16.0 \mathrm{mg}, 32 \mu \mathrm{mol}, 16 \mathrm{~mol} \%\right.$ ). The tube backfilled with $\mathrm{N}_{2}$. The flask was added freshly distilled toluene $(4.0 \mathrm{~mL})$, and stirred for $15 \mathrm{~min}$. Then the allyl alcohols $1(0.2 \mathrm{mmol}, 1.0$ equiv), secondary amines 2 (0.8 mmol, 4.0 equiv.), and $\mathrm{Sc}(\mathrm{OTf})_{3}(98 \mathrm{mg}, 0.2 \mathrm{mmol}, 100 \mathrm{~mol} \%$ ) were added. The tube was sealed and stirred at rt. After the reaction was complete (monitored by TLC), the solution was quenched with water $(10 \mathrm{~mL})$ and extracted with EA $(3 \times 10 \mathrm{~mL})$. The organic layers dried over $\mathrm{Na}_{2} \mathrm{SO}_{4}$ and concentrated by rotary evaporation. The crude product was purified by silica gel column chromatography $(\mathrm{DCM} / \mathrm{PE}=1 / 5-1 / 10)$ to afford the desired product (4aa-4an, 7aa-7ae).

General procedure $\mathbf{C}$ : a flame dried tube was cooled to rt. and charged with $[\operatorname{Ir}(\operatorname{cod}) \mathrm{Cl}]_{2}(5.2 \mathrm{mg}, 8$ $\mu \mathrm{mol}, 4 \mathrm{~mol} \%$ ) and ligand $\mathbf{L 1}$ (16.0 mg, $32 \mu \mathrm{mol}, 16 \mathrm{~mol} \%$ ). The tube backfilled with $\mathrm{N}_{2}$. The flask was added freshly distilled toluene $(4.0 \mathrm{~mL})$, and stirred for $15 \mathrm{~min}$. Then the allyl alcohols $1(0.2 \mathrm{mmol}, 1.0$ equiv), secondary amines 2 (0.8 mmol, 4.0 equiv.), and $\mathrm{Sc}(\mathrm{OTf})_{3}(98 \mathrm{mg}, 0.2 \mathrm{mmol}, 100 \mathrm{~mol} \%$ ) were added. The tube was sealed and stirred at $80^{\circ} \mathrm{C}$. After the reaction was complete (monitored by TLC), the solution was quenched with water $(10 \mathrm{~mL})$ and extracted with EA $(3 \times 10 \mathrm{~mL})$. The organic layers dried over $\mathrm{Na}_{2} \mathrm{SO}_{4}$ and concentrated by rotary evaporation. The crude product was purified by silica gel column chromatography $(\mathrm{DCM} / \mathrm{PE}=1 / 2-1 / 6)$ to afford the desired product (5aa-5ai).

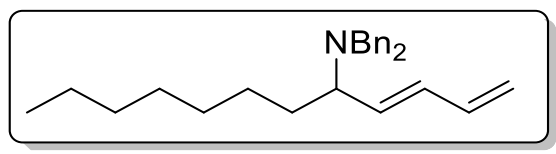

\section{(3E)- $N, N$-Dibenzyl-dodeca-1,3-dien-5-amine (3aa)}

The title compound was prepared via general procedure A, purified by flash chromatography on silica gel eluting with in $\mathrm{CH}_{2} \mathrm{Cl}_{2} / \mathrm{PE}=1 / 3(\mathrm{Rf}=0.32)$, colorless oil, $63.8 \mathrm{mg}, 88 \%$ yield, ${ }^{1} \mathrm{H} \mathrm{NMR}(400 \mathrm{MHz}$, $\left.\mathrm{CDCl}_{3}\right) \delta 7.38(\mathrm{~d}, J=7.6 \mathrm{~Hz}, 4 \mathrm{H}), 7.29(\mathrm{~d}, J=7.6 \mathrm{~Hz}, 4 \mathrm{H}), 7.21(\mathrm{t}, J=7.6 \mathrm{~Hz}, 2 \mathrm{H}), 6.39(\mathrm{dt}, J=17.2$, $10.4 \mathrm{~Hz}, 1 \mathrm{H}), 6.04(\mathrm{dd}, J=15.2,10.4 \mathrm{~Hz}, 1 \mathrm{H}), 5.70(\mathrm{dd}, J=15.2,8.8 \mathrm{~Hz}, 1 \mathrm{H}), 5.19$ (d, $J=17.2 \mathrm{~Hz}, 1 \mathrm{H})$, $5.07(\mathrm{~d}, J=10.4 \mathrm{~Hz}, 1 \mathrm{H}), 3.79(\mathrm{~d}, J=14.0 \mathrm{~Hz}, 2 \mathrm{H}), 3.35(\mathrm{~d}, J=14.0 \mathrm{~Hz}, 2 \mathrm{H}), 3.03(\mathrm{dd}, J=15.2,7.2 \mathrm{~Hz}$, $1 \mathrm{H}), 1.71-1.64(\mathrm{~m}, 1 \mathrm{H}), 1.43-1.14(\mathrm{~m}, 11 \mathrm{H}), 0.87(\mathrm{t}, J=7.2 \mathrm{~Hz}, 3 \mathrm{H}) .{ }^{13} \mathrm{C} \mathrm{NMR}\left(100 \mathrm{MHz}, \mathrm{CDCl}_{3}\right) \delta$ 140.7, 137.0, 133. 8, 133.3, 128.8, 128.3, 126.8, 116.4, 59.9, 53.8, 32.5, 32.0, 29.7, 29.4, 26.6, 22.8, 14.3. HRMS (ESI) calculated for $\mathrm{C}_{26} \mathrm{H}_{36} \mathrm{~N}[\mathrm{M}+\mathrm{H}]^{+}:$362.2848, found: 362.2838 .

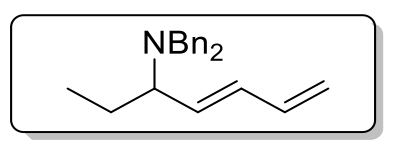

(3E)-N,N-Dibenzyl-hepta-1,3-dien-5-amine (3ab)

The title compound was prepared via general procedure A, purified by flash chromatography on silica gel 
eluting with in $\mathrm{CH}_{2} \mathrm{Cl}_{2} / \mathrm{PE}=1 / 3(\mathrm{Rf}=0.35)$, colorless oil, $39.0 \mathrm{mg}$, 68\% yield, ${ }^{1} \mathrm{H} \mathrm{NMR}(300 \mathrm{MHz}$, $\left.\mathrm{CDCl}_{3}\right) \delta 7.38(\mathrm{~d}, J=7.2 \mathrm{~Hz}, 4 \mathrm{H}), 7.33-7.17(\mathrm{~m}, 6 \mathrm{H}), 6.39(\mathrm{dt}, J=17.1,10.2 \mathrm{~Hz}, 1 \mathrm{H}), 6.04(\mathrm{dd}, J=15.0$, $10.5 \mathrm{~Hz}, 1 \mathrm{H}), 5.70$ (dd, $J=15.0,8.7 \mathrm{~Hz}, 1 \mathrm{H}), 5.19$ (dd, $J=17.1,1.6 \mathrm{~Hz}, 1 \mathrm{H}), 5.07$ (dd, $J=10.2,1.5 \mathrm{~Hz}$, $1 \mathrm{H}), 3.79(\mathrm{~d}, J=13.8 \mathrm{~Hz}, 2 \mathrm{H}), 3.37$ (d, $J=13.8 \mathrm{~Hz}, 2 \mathrm{H}), 2.95$ (dd, $J=15.9,7.5 \mathrm{~Hz}, 1 \mathrm{H}), 1.81-1.61$ (m, $1 \mathrm{H}), 1.59-1.37(\mathrm{~m}, 1 \mathrm{H}), 0.89(\mathrm{t}, J=7.2 \mathrm{~Hz}, 3 \mathrm{H}) .{ }^{13} \mathrm{C} \mathrm{NMR}\left(75 \mathrm{MHz}, \mathrm{CDCl}_{3}\right) \delta 140.7,137.0,133.9,133.0$, 128.8, 128.3, 126.8, 116.4, 61.8, 53.8, 25.4, 11.5. HRMS (ESI) calculated for $\mathrm{C}_{21} \mathrm{H}_{26} \mathrm{~N}[\mathrm{M}+\mathrm{H}]^{+}: 292.2065$, found: 292.2062 .

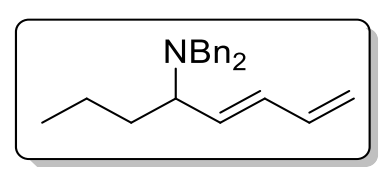

(3E)- $N, N$-Dibenzyl-octa-1,3-dien-5-amine (3ac)

The title compound was prepared via general procedure A, purified by flash chromatography on silica gel eluting with in $\mathrm{CH}_{2} \mathrm{Cl}_{2} / \mathrm{PE}=1 / 3(\mathrm{Rf}=0.30)$, colorless oil, $46.3 \mathrm{mg}, 76 \%$ yield, ${ }^{1} \mathrm{H} \mathrm{NMR}(400 \mathrm{MHz}$, $\left.\mathrm{CDCl}_{3}\right) \delta 7.38(\mathrm{~d}, J=7.2 \mathrm{~Hz}, 4 \mathrm{H}), 7.30(\mathrm{t}, J=7.2 \mathrm{~Hz}, 4 \mathrm{H}), 7.21(\mathrm{t}, J=7.2 \mathrm{~Hz}, 2 \mathrm{H}), 6.39(\mathrm{dt}, J=17.2$, $10.4 \mathrm{~Hz}, 1 \mathrm{H}), 6.04(\mathrm{dd}, J=15.2,10.4 \mathrm{~Hz}, 1 \mathrm{H}), 5.71(\mathrm{dd}, J=15.2,8.8 \mathrm{~Hz}, 1 \mathrm{H}), 5.19(\mathrm{~d}, J=17.2 \mathrm{~Hz}, 1 \mathrm{H})$, $5.07(\mathrm{~d}, J=10.2 \mathrm{~Hz}, 1 \mathrm{H}), 3.79(\mathrm{~d}, J=13.6 \mathrm{~Hz}, 2 \mathrm{H}), 3.36(\mathrm{~d}, J=13.6 \mathrm{~Hz}, 2 \mathrm{H}), 3.05(\mathrm{q}, 7.2 \mathrm{~Hz}, 1 \mathrm{H}), 1.78-$ $1.60(\mathrm{~m}, 1 \mathrm{H}), 1.46-1.34(\mathrm{~m}, 2 \mathrm{H}), 1.32-1.24(\mathrm{~m}, 1 \mathrm{H}), 0.80(\mathrm{t}, J=7.2 \mathrm{~Hz}, 3 \mathrm{H}) .{ }^{13} \mathrm{C} \mathrm{NMR}\left(100 \mathrm{MHz}, \mathrm{CDCl}_{3}\right)$ $\delta 140.7,137.0,133.8,133.2,128.8,128.3,126.8,116.4,59.7,53.8,34.8,19.9$, 14.2. HRMS (ESI) calculated for $\mathrm{C}_{22} \mathrm{H}_{28} \mathrm{~N}[\mathrm{M}+\mathrm{H}]^{+}: 306.2222$, found: 306.2218 .

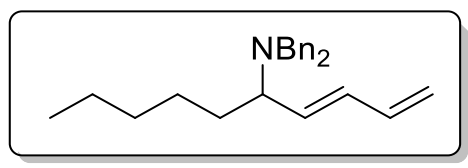

\section{(3E)- $N, N$-Dibenzyl-deca-1,3-dien-5-amine (3ad)}

The title compound was prepared via general procedure A, purified by flash chromatography on silica gel eluting with in $\mathrm{CH}_{2} \mathrm{Cl}_{2} / \mathrm{PE}=1 / 3(\mathrm{Rf}=0.40)$, colorless oil, $54.6 \mathrm{mg}, 82 \%$ yield, ${ }^{1} \mathrm{H} \mathrm{NMR}(400 \mathrm{MHz}$, $\left.\mathrm{CDCl}_{3}\right) \delta 7.38(\mathrm{~d}, J=7.2 \mathrm{~Hz}, 4 \mathrm{H}), 7.30(\mathrm{t}, J=7.2 \mathrm{~Hz}, 4 \mathrm{H}), 7.21(\mathrm{t}, J=7.2 \mathrm{~Hz}, 2 \mathrm{H}), 6.39$ (dt, $J=17.2$, $10.4 \mathrm{~Hz}, 1 \mathrm{H}), 6.04(\mathrm{dd}, J=15.2,10.4 \mathrm{~Hz}, 1 \mathrm{H}), 5.70(\mathrm{dd}, J=15.2,8.8 \mathrm{~Hz}, 1 \mathrm{H}), 5.19(\mathrm{~d}, J=17.2 \mathrm{~Hz}, 1 \mathrm{H})$, $5.08(\mathrm{~d}, J=10.4 \mathrm{~Hz}, 1 \mathrm{H}), 3.79$ (d, $J=14.0 \mathrm{~Hz}, 2 \mathrm{H}), 3.36$ (d, $J=14.0 \mathrm{~Hz}, 2 \mathrm{H}), 3.03$ (dd, $J=15.6,7.2 \mathrm{~Hz}$, $1 \mathrm{H}), 1.74-1.63(\mathrm{~m}, 1 \mathrm{H}), 1.46-1.10(\mathrm{~m}, 7 \mathrm{H}), 0.84(\mathrm{t}, J=7.2 \mathrm{~Hz}, 3 \mathrm{H}) .{ }^{13} \mathrm{C} \mathrm{NMR}\left(100 \mathrm{MHz}, \mathrm{CDCl}_{3}\right) \delta 140.7$, 137.0, 133.8, 133.3, 128.8, 128.3, 126.8, 116.4, 59.8, 53.8, 32.5, 31.9, 26.3, 22.8, 14.2. HRMS (ESI) calculated for $\mathrm{C}_{24} \mathrm{H}_{32} \mathrm{~N}[\mathrm{M}+\mathrm{H}]^{+}: 334.2535$, found: 334.2543 .

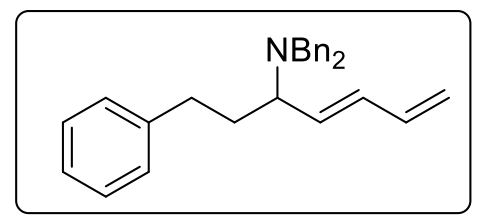

(3E)- $N, N$-Dibenzyl-7-phenyl-hepta-1,3-dien-5-amine (3ae) 
The title compound was prepared via general procedure A, purified by flash chromatography on silica gel eluting with in $\mathrm{CH}_{2} \mathrm{Cl}_{2} / \mathrm{PE}=1 / 3(\mathrm{Rf}=0.30)$, colorless oil, $58.5 \mathrm{mg}, 80 \%$ yield, ${ }^{1} \mathrm{H}$ NMR $(300 \mathrm{MHz}$, $\left.\mathrm{CDCl}_{3}\right) \delta$ 7.42-7.35 (m, 4H), 7.33-7.17 (m, 8H), 7.15-7.03 (m, 3H), 6.40 (dt, $\left.J=16.8,10.2 \mathrm{~Hz}, 1 \mathrm{H}\right), 6.07$ (dd, $J=15.6,10.5 \mathrm{~Hz}, 1 \mathrm{H}), 5.75$ (dd, $J=15.6,8.7 \mathrm{~Hz}, 1 \mathrm{H}), 5.21$ (dd, $J=17.1,1.5 \mathrm{~Hz}, 1 \mathrm{H}), 5.11-5.00$ (m, $1 \mathrm{H}), 3.82(\mathrm{~d}, J=13.8 \mathrm{~Hz}, 2 \mathrm{H}), 3.39(\mathrm{~d}, J=13.8 \mathrm{~Hz}, 2 \mathrm{H}), 3.14(\mathrm{dd}, J=15.6,7.2 \mathrm{~Hz}, 1 \mathrm{H}), 2.77(\mathrm{ddd}, J=$ $13.8,10.5,5.7 \mathrm{~Hz}, 1 \mathrm{H}), 2.51(\mathrm{ddd}, J=13.8,10.5,5.7 \mathrm{~Hz}, 1 \mathrm{H}), 2.08-1.96(\mathrm{~m}, 1 \mathrm{H}), 1.87-1.65(\mathrm{~m}, 1 \mathrm{H}) .{ }^{13} \mathrm{C}$ $\operatorname{NMR}\left(75 \mathrm{MHz}, \mathrm{CDCl}_{3}\right) \delta 142.7,140.5,136.9,134.2,132.6,128.9,128.5,128.4,128.3,126.9,125.7$, 116.8, 59.8, 53.9, 34.6, 33.1. HRMS (ESI) calculated for $\mathrm{C}_{27} \mathrm{H}_{30} \mathrm{~N}[\mathrm{M}+\mathrm{H}]^{+}$: 368.2378, found: 368.2377 .

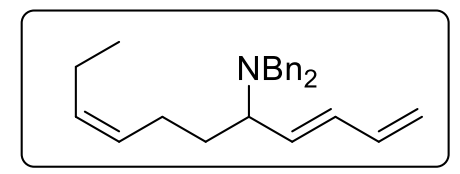

\section{(3E, $8 Z$ )- $N, N$-Dibenzyl-undeca-1,3,8-trien-5-amine (3af)}

The title compound was prepared via general procedure A, purified by flash chromatography on silica gel eluting with in $\mathrm{CH}_{2} \mathrm{Cl}_{2} / \mathrm{PE}=1 / 3(\mathrm{Rf}=0.26)$, colorless oil, $38.4 \mathrm{mg}, 56 \%$ yield, ${ }^{1} \mathrm{H} \mathrm{NMR}(400 \mathrm{MHz}$, $\left.\mathrm{CDCl}_{3}\right) \delta$ 7.43-7.07 (m, 10H), $6.39(\mathrm{dt}, J=17.2,10.4 \mathrm{~Hz}, 1 \mathrm{H}), 6.05(\mathrm{dd}, J=15.2,10.4 \mathrm{~Hz}, 1 \mathrm{H}), 5.71(\mathrm{dd}$, $J=15.2,8.8 \mathrm{~Hz}, 1 \mathrm{H}), 5.32-5.17(\mathrm{~m}, 3 \mathrm{H}), 5.19(\mathrm{~d}, J=17.2 \mathrm{~Hz}, 1 \mathrm{H}), 3.79(\mathrm{~d}, J=14.0 \mathrm{~Hz}, 2 \mathrm{H}), 3.37(\mathrm{~d}, J$ $=14.0 \mathrm{~Hz}, 2 \mathrm{H}), 3.07(\mathrm{q}, J=7.2 \mathrm{~Hz}, 1 \mathrm{H}), 2.22-2.11(\mathrm{~m}, 1 \mathrm{H}), 2.05-1.85(\mathrm{~m}, 3 \mathrm{H}), 1.79-1.71(\mathrm{~m}, 1 \mathrm{H}), 1.53-$ $1.45(\mathrm{~m}, 1 \mathrm{H}), 0.91(\mathrm{t}, J=7.6 \mathrm{~Hz}, 3 \mathrm{H}) .{ }^{13} \mathrm{C} \mathrm{NMR}\left(100 \mathrm{MHz}, \mathrm{CDCl}_{3}\right) \delta 140.5,136.9,134.0,132.8,131.9$, 128.9, 128.8, 128.3, 126.8, 116.6, 59.8, 53.9, 32.7, 24.4, 20.6, 14.5. HRMS (ESI) calculated for $\mathrm{C}_{25} \mathrm{H}_{32} \mathrm{~N}$ $[\mathrm{M}+\mathrm{H}]^{+}:$346.2535, found: 346.2531 .

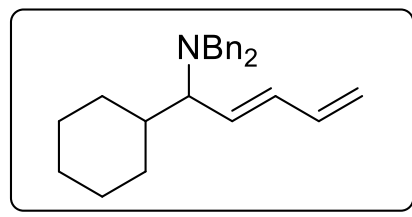

(3E)- $N, N$-Dibenzyl-5-cyclohexyl-penta-1,3-dien-5-amine (3ag)

The title compound was prepared via general procedure A, purified by flash chromatography on silica gel eluting with in $\mathrm{CH}_{2} \mathrm{Cl}_{2} / \mathrm{PE}=1 / 3(\mathrm{Rf}=0.26)$, colorless oil, $33.2 \mathrm{mg}, 48 \%$ yield, ${ }^{1} \mathrm{H} \mathrm{NMR}(400 \mathrm{MHz}$, $\left.\mathrm{CDCl}_{3}\right) \delta 7.39(\mathrm{~d}, J=7.2 \mathrm{~Hz}, 4 \mathrm{H}), 7.31(\mathrm{t}, J=7.6 \mathrm{~Hz}, 4 \mathrm{H}), 7.22(\mathrm{t}, J=7.2 \mathrm{~Hz}, 2 \mathrm{H}), 6.41(\mathrm{dt}, J=17.2$, $10.4 \mathrm{~Hz}, 1 \mathrm{H}), 5.95(\mathrm{dd}, J=15.2,10.4 \mathrm{~Hz}, 1 \mathrm{H}), 5.61(\mathrm{dd}, J=15.2,10.0 \mathrm{~Hz}, 1 \mathrm{H}), 5.20(\mathrm{~d}, J=17.2 \mathrm{~Hz}$, $1 \mathrm{H}), 5.08(\mathrm{~d}, J=10.0 \mathrm{~Hz}, 1 \mathrm{H}), 3.85(\mathrm{~d}, J=14.0 \mathrm{~Hz}, 2 \mathrm{H}), 3.28(\mathrm{~d}, J=14.0 \mathrm{~Hz}, 2 \mathrm{H}), 2.63(\mathrm{t}, J=10.0 \mathrm{~Hz}$, $1 \mathrm{H}), 2.4-2.39(\mathrm{~m}, 1 \mathrm{H}), 1.73-1.69(\mathrm{~m}, 1 \mathrm{H}), 1.63-1.49(\mathrm{~m}, 4 \mathrm{H}), 1.27-0.97(\mathrm{~m}, 3 \mathrm{H}), 0.87-0.71(\mathrm{~m}, 1 \mathrm{H})$, 0.69-0.55 (m, 1H). $\left.{ }^{13} \mathrm{C} \mathrm{NMR} \mathrm{(100} \mathrm{MHz,} \mathrm{CDCl}_{3}\right) \delta 140.6,136.8,135.3,131.8,128.8,128.3,126.7,116.3$, $65.9,53.8,38.9,31.5,30.6,26.8,26.4(2 \mathrm{C})$. HRMS (ESI) calculated for $\mathrm{C}_{25} \mathrm{H}_{32} \mathrm{~N}[\mathrm{M}+\mathrm{H}]^{+}: 346.2535$, found: 346.2531 . 


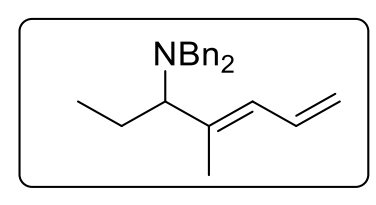

(3E)- $N, N$-Dibenzyl-4-methyl-hepta-1,3-dien-5-amine (3ah)

The title compound was prepared via general procedure A, purified by flash chromatography on silica gel eluting with in $\mathrm{CH}_{2} \mathrm{Cl}_{2} / \mathrm{PE}=1 / 3(\mathrm{Rf}=0.38)$, colorless oil, $39.8 \mathrm{mg}$, 65\% yield, ${ }^{1} \mathrm{H} \mathrm{NMR}(400 \mathrm{MHz}$, $\left.\mathrm{CDCl}_{3}\right) \delta 7.40-7.04(\mathrm{~m}, 10 \mathrm{H}), 6.64(\mathrm{dt}, J=16.8,10.0 \mathrm{~Hz}, 1 \mathrm{H}), 5.84(\mathrm{~d}, J=10.8 \mathrm{~Hz}, 1 \mathrm{H}), 5.14(\mathrm{~d}, J=16.8$ $\mathrm{Hz}, 1 \mathrm{H}), 5.06(\mathrm{~d}, J=10.0 \mathrm{~Hz}, 1 \mathrm{H}), 3.67(\mathrm{~d}, J=14.0 \mathrm{~Hz}, 2 \mathrm{H}), 3.52(\mathrm{~d}, J=14.0 \mathrm{~Hz}, 2 \mathrm{H}), 2.90(\mathrm{dd}, J=9.6$, $4.8 \mathrm{~Hz}, 1 \mathrm{H}), 1.86-1.71(\mathrm{~m}, 1 \mathrm{H}), 1.80(\mathrm{~s}, 3 \mathrm{H}), 1.56-1.45(\mathrm{~m}, 1 \mathrm{H}), 0.81(\mathrm{t}, J=7.3 \mathrm{~Hz}, 3 \mathrm{H}) .{ }^{13} \mathrm{C}$ NMR $(75$ $\left.\mathrm{MHz}_{\mathrm{CDCl}}\right) \delta 140.9,137.8,133.2,128.9,128.5,128.2,126.7,116.1,67.8,54.4,20.5,15.4,11.8$. HRMS (ESI) calculated for $\mathrm{C}_{22} \mathrm{H}_{28} \mathrm{~N}[\mathrm{M}+\mathrm{H}]^{+}: 306.2222$, found: 306.2220 .

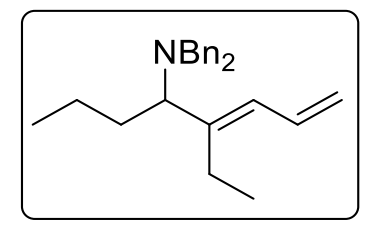

(3E)- $N, N$-Dibenzyl-4-ethyl-octa-1,3-dien-5-amine (3ai)

The title compound was prepared via general procedure A, purified by flash chromatography on silica gel eluting with in $\mathrm{CH}_{2} \mathrm{Cl}_{2} / \mathrm{PE}=1 / 3(\mathrm{Rf}=0.32)$, colorless oil, $54.8 \mathrm{mg}, 82 \%$ yield, ${ }^{1} \mathrm{H} \mathrm{NMR}(400 \mathrm{MHz}$, $\left.\mathrm{CDCl}_{3}\right) \delta 7.39-7.10(\mathrm{~m}, 10 \mathrm{H}), 6.64(\mathrm{dt}, J=16.8,10.4 \mathrm{~Hz}, 1 \mathrm{H}), 5.83(\mathrm{~d}, J=10.8 \mathrm{~Hz}, 1 \mathrm{H}), 5.14(\mathrm{dd}, J=$ $16.8,1.6 \mathrm{~Hz}, 1 \mathrm{H}), 5.04(\mathrm{dd}, J=10.4,1.6 \mathrm{~Hz}, 1 \mathrm{H}), 3.67$ (d, $J=13.6 \mathrm{~Hz}, 2 \mathrm{H}), 3.45$ (d, $J=13.6 \mathrm{~Hz}, 2 \mathrm{H})$, $3.05(\mathrm{dd}, J=10.4,3.6 \mathrm{~Hz}, 1 \mathrm{H}), 2.39-2.12(\mathrm{~m}, 2 \mathrm{H}), 1.82-1.65(\mathrm{~m}, 1 \mathrm{H}), 1.61-1.48(\mathrm{~m}, 1 \mathrm{H}), 1.35-1.20(\mathrm{~m}$, $1 \mathrm{H}), 1.17-1.08(\mathrm{~m}, 1 \mathrm{H}), 0.88(\mathrm{t}, J=7.4 \mathrm{~Hz}, 3 \mathrm{H}), 0.73(\mathrm{t}, J=7.6 \mathrm{~Hz}, 3 \mathrm{H}) .{ }^{13} \mathrm{C} \mathrm{NMR}\left(100 \mathrm{MHz}, \mathrm{CDCl}_{3}\right) \delta$ $144.4,140.8,133.3,129.1,128.2,126.8,126.3,116.1,60.9,53.8,26.5,22.9,20.3,14.4$, 13.8. HRMS (ESI) calculated for $\mathrm{C}_{24} \mathrm{H}_{32} \mathrm{~N}[\mathrm{M}+\mathrm{H}]^{+}: 334.2535$, found: 334.2543 .

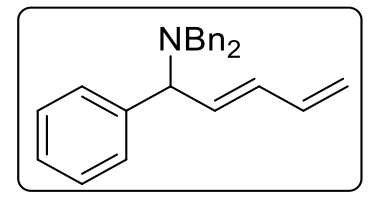

(2E)-N,N-dibenzyl-5-phenyl-penta-1,3-dien-5-amine (3aj)

The title compound was prepared via general procedure A, purified by flash chromatography on silica gel eluting with in $\mathrm{CH}_{2} \mathrm{Cl}_{2} / \mathrm{PE}=1 / 3(\mathrm{Rf}=0.30)$, yellow oil, $54.2 \mathrm{mg}, 79 \%$ yield, ${ }^{1} \mathrm{H} \mathrm{NMR}\left(300 \mathrm{MHz}, \mathrm{CDCl}_{3}\right)$ $\delta 7.48(\mathrm{~d}, J=7.5 \mathrm{~Hz}, 2 \mathrm{H}), 7.41(\mathrm{~d}, J=7.5 \mathrm{~Hz}, 4 \mathrm{H}), 7.36-7.16(\mathrm{~m}, 9 \mathrm{H}), 6.46(\mathrm{dt}, J=16.8,10.2 \mathrm{~Hz}, 1 \mathrm{H})$, $6.18(\mathrm{dd}, J=15.3,10.2 \mathrm{~Hz}, 1 \mathrm{H}), 5.97(\mathrm{dd}, J=15.3,8.7 \mathrm{~Hz}, 1 \mathrm{H}), 5.22(\mathrm{~d}, J=16.8 \mathrm{~Hz}, 1 \mathrm{H}), 5.12(\mathrm{dd}, J=$ 10.2, $1.2 \mathrm{~Hz}, 1 \mathrm{H}), 4.31(\mathrm{~d}, J=8.7 \mathrm{~Hz}, 1 \mathrm{H}), 3.66(\mathrm{~d}, J=13.8 \mathrm{~Hz}, 2 \mathrm{H}), 3.53(\mathrm{~d}, J=13.8 \mathrm{~Hz}, 2 \mathrm{H}) .{ }^{13} \mathrm{C} \mathrm{NMR}$ $\left(75 \mathrm{MHz}, \mathrm{CDCl}_{3}\right) \delta 141.6,140.1,136.7,135.3,131.7,128.7,128.4,128.3,127.1,126.9(2 \mathrm{C}), 117.3,64.4$, 53.9. HRMS (ESI) calculated for $\mathrm{C}_{25} \mathrm{H}_{26} \mathrm{~N}[\mathrm{M}+\mathrm{H}]^{+}: 340.2065$, found: 340.2053 . 


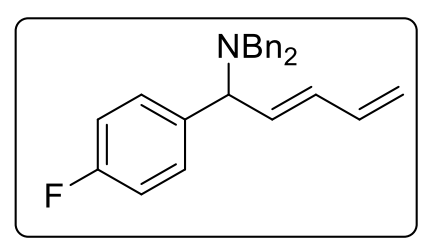

\section{(2E)- $N, N$-dibenzyl-5-p-flurophenyl-penta-1,3-dien-5-amine (3ak)}

The title compound was prepared via general procedure A, purified by flash chromatography on silica gel eluting with in $\mathrm{CH}_{2} \mathrm{Cl}_{2} / \mathrm{PE}=1 / 3(\mathrm{Rf}=0.32)$, yellow oil, $54.7 \mathrm{mg}, 76 \%$ yield, ${ }^{1} \mathrm{H} \mathrm{NMR}\left(400 \mathrm{MHz}, \mathrm{CDCl}_{3}\right)$ $\delta$ 7.46-7.37 (m, 6H), 7.33-7.29 (m, 4H), $7.22(\mathrm{t}, J=7.2 \mathrm{~Hz}, 2 \mathrm{H}), 7.09-6.85(\mathrm{~m}, 2 \mathrm{H}), 6.46(\mathrm{dt}, J=17.2$, $10.4 \mathrm{~Hz}, 1 \mathrm{H}), 6.17$ (dd, $J=15.2,10.4 \mathrm{~Hz}, 1 \mathrm{H}), 5.94(\mathrm{dd}, J=15.2,8.8 \mathrm{~Hz}, 1 \mathrm{H}), 5.24(\mathrm{~d}, J=17.2 \mathrm{~Hz}, 1 \mathrm{H})$, $5.14(\mathrm{~d}, J=10.4 \mathrm{~Hz}, 1 \mathrm{H}), 4.27(\mathrm{~d}, J=8.8 \mathrm{~Hz}, 1 \mathrm{H}), 3.64(\mathrm{~d}, J=14.0 \mathrm{~Hz}, 2 \mathrm{H}), 3.51(\mathrm{~d}, J=14.0 \mathrm{~Hz}, 2 \mathrm{H})$. ${ }^{19} \mathrm{~F}$ NMR $\left(282 \mathrm{MHz}, \mathrm{CDCl}_{3}\right) \delta-115.95 .{ }^{13} \mathrm{C} \mathrm{NMR}\left(100 \mathrm{MHz}, \mathrm{CDCl}_{3}\right) \delta 162.04(\mathrm{~d}, J=245.2 \mathrm{~Hz}), 139.94$, $137.41(\mathrm{~d}, J=3.2 \mathrm{~Hz}), 136.5,135.5,131.2,129.7$ (d, $J=8.0 \mathrm{~Hz}), 128.7,128.4,127.0,117.6,115.1(\mathrm{~d}, J$ $=21.2 \mathrm{~Hz}), 63.8,53.9$. HRMS (ESI) calculated for $\mathrm{C}_{25} \mathrm{H}_{25} \mathrm{FN}[\mathrm{M}+\mathrm{H}]^{+}:$358.1971, found: 358.1975.

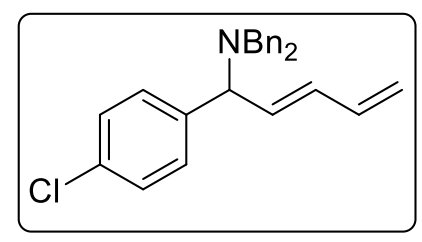

\section{(2E)- $N, N$-dibenzyl-5-p-chlorophenyl-penta-1,3-dien-5-amine (3al)}

The title compound was prepared via general procedure A, purified by flash chromatography on silica gel eluting with in $\mathrm{CH}_{2} \mathrm{Cl}_{2} / \mathrm{PE}=1 / 3(\mathrm{Rf}=0.35)$, colorless oil, $56.0 \mathrm{mg}, 75 \%$ yield, ${ }^{1} \mathrm{H}$ NMR (400 MHz, $\left.\mathrm{CDCl}_{3}\right) \delta$ 7.44-7.21 (m, 14H), $6.46(\mathrm{dt}, J=17.2,10.4 \mathrm{~Hz}, 1 \mathrm{H}), 6.17(\mathrm{dd}, J=15.2,10.4 \mathrm{~Hz}, 1 \mathrm{H}), 5.93(\mathrm{dd}$, $J=15.2,8.8 \mathrm{~Hz}, 1 \mathrm{H}), 5.25(\mathrm{~d}, J=16.8 \mathrm{~Hz}, 1 \mathrm{H}), 5.15(\mathrm{~d}, J=10.4 \mathrm{~Hz}, 1 \mathrm{H}), 4.26(\mathrm{~d}, J=8.8 \mathrm{~Hz}, 1 \mathrm{H}), 3.64$ $(\mathrm{d}, J=14.0 \mathrm{~Hz}, 2 \mathrm{H}), 3.51(\mathrm{~d}, J=14.0 \mathrm{~Hz}, 2 \mathrm{H}) .{ }^{13} \mathrm{C} \mathrm{NMR}\left(100 \mathrm{MHz}, \mathrm{CDCl}_{3}\right) \delta 140.3,139.8,136.5,135.7$, 132.8, 130.9, 129.6, 128.7, 128.4(2C), 127.1, 117.7, 63.9, 53.9. HRMS (ESI) calculated for $\mathrm{C}_{25} \mathrm{H}_{25} \mathrm{ClN}$ $[\mathrm{M}+\mathrm{H}]^{+}:$374.1676, found: 374.1674 .

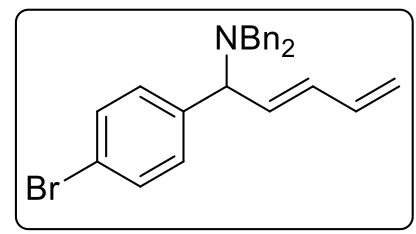

(2E)- $N, N$-dibenzyl-5-p-bromophenyl-penta-1,3-dien-5-amine (3am)

The title compound was prepared via general procedure A, purified by flash chromatography on silica gel eluting with in $\mathrm{CH}_{2} \mathrm{Cl}_{2} / \mathrm{PE}=1 / 3(\mathrm{Rf}=0.35)$, pale yellow oil, $56.0 \mathrm{mg}, 67 \%$ yield, ${ }^{1} \mathrm{H} \mathrm{NMR}(300 \mathrm{MHz}$, $\left.\mathrm{CDCl}_{3}\right) \delta$ 7.48-7.42 (m, 2H), 7.40-7.28 (m, 10H), 7.26-7.18 (m, 2H), $6.46(\mathrm{dt}, J=16.8,10.2 \mathrm{~Hz}, 1 \mathrm{H})$, $6.17(\mathrm{dd}, J=15.3,10.2 \mathrm{~Hz}, 1 \mathrm{H}), 5.92(\mathrm{dd}, J=15.3,8.7 \mathrm{~Hz}, 1 \mathrm{H}), 5.24(\mathrm{dd}, J=16.8,1.5 \mathrm{~Hz}, 1 \mathrm{H}), 5.15$ $(\mathrm{dd}, J=10.2,1.5 \mathrm{~Hz}, 1 \mathrm{H}), 4.24(\mathrm{~d}, J=8.7 \mathrm{~Hz}, 1 \mathrm{H}), 3.64(\mathrm{~d}, J=13.8 \mathrm{~Hz}, 2 \mathrm{H}), 3.50(\mathrm{~d}, J=13.8 \mathrm{~Hz}, 2 \mathrm{H})$. 
${ }^{13} \mathrm{C} \mathrm{NMR}\left(75 \mathrm{MHz}, \mathrm{CDCl}_{3}\right) \delta 140.9,139.8,136.4,135.8,131.4,130.8,130.0,128.7,128.5,127.1,121.0$, 117.7, 64.0, 53.9. HRMS (ESI) calculated for $\mathrm{C}_{25} \mathrm{H}_{25} \mathrm{BrN}[\mathrm{M}+\mathrm{H}]^{+}$: 418.1170, found: 418.1163.

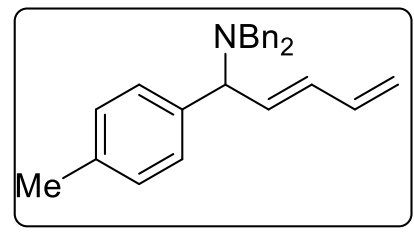

\section{(2E)- $N, N$-dibenzyl-5-p-methylphenyl-penta-1,3-dien-5-amine (3an)}

The title compound was prepared via general procedure A, purified by flash chromatography on silica gel eluting with in $\mathrm{CH}_{2} \mathrm{Cl}_{2} / \mathrm{PE}=1 / 3(\mathrm{Rf}=0.31)$, colorless oil, $54.1 \mathrm{mg}, 76 \%$ yield, ${ }^{1} \mathrm{H}$ NMR $(300 \mathrm{MHz}$, $\left.\mathrm{CDCl}_{3}\right) \delta$ 7.43-7.26 (m, 10H), 7.24-7.12 (m, 4H), $6.45(\mathrm{dt}, J=17.1,10.2 \mathrm{~Hz}, 1 \mathrm{H}), 6.17(\mathrm{dd}, J=15.3,10.2$ $\mathrm{Hz}, 1 \mathrm{H}), 5.96(\mathrm{dd}, J=15.3,8.4 \mathrm{~Hz}, 1 \mathrm{H}), 5.21(\mathrm{dd}, J=17.1,1.5 \mathrm{~Hz}, 1 \mathrm{H}), 5.11(\mathrm{dd}, J=10.2,1.5 \mathrm{~Hz}, 1 \mathrm{H})$, $4.28(\mathrm{~d}, J=8.4 \mathrm{~Hz}, 1 \mathrm{H}), 3.64(\mathrm{~d}, J=13.8 \mathrm{~Hz}, 2 \mathrm{H}), 3.54(\mathrm{~d}, J=13.8 \mathrm{~Hz}, 2 \mathrm{H}), 2.33(\mathrm{~s}, 3 \mathrm{H}) .{ }^{13} \mathrm{C} \mathrm{NMR}(75$ $\left.\mathrm{MHz}, \mathrm{CDCl}_{3}\right) \delta 140.2,138.4,136.8,136.7,134.9,132.3,129.0,128.8,128.4,128.3,126.9,117.1,64.2$, 53.9, 21.2. HRMS (ESI) calculated for $\mathrm{C}_{26} \mathrm{H}_{28} \mathrm{~N}[\mathrm{M}+\mathrm{H}]^{+}: 354.2222$, found: 354.2224.

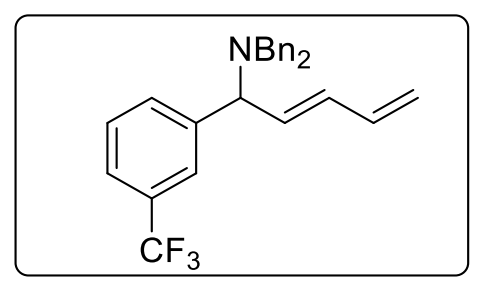

(2E)- $N, N$-Dibenzyl-5- $m$-trifluromethylphenyl-penta-1,3-dien-5-amine (3ao)

The title compound was prepared via general procedure A, purified by flash chromatography on silica gel eluting with in $\mathrm{CH}_{2} \mathrm{Cl}_{2} / \mathrm{PE}=1 / 3(\mathrm{Rf}=0.28)$, colorless oil, $55.8 \mathrm{mg}, 68 \%$ yield, ${ }^{1} \mathrm{H} \mathrm{NMR}(300 \mathrm{MHz}$, $\left.\mathrm{CDCl}_{3}\right) \delta 7.79(\mathrm{~s}, 1 \mathrm{H}), 7.74(\mathrm{~d}, J=7.6 \mathrm{~Hz}, 1 \mathrm{H}), 7.55(\mathrm{~d}, J=7.6 \mathrm{~Hz}, 1 \mathrm{H}), 7.49(\mathrm{t}, J=7.6 \mathrm{~Hz}, 1 \mathrm{H}), 7.43(\mathrm{~d}$, $J=7.2 \mathrm{~Hz}, 4 \mathrm{H}), 7.37(\mathrm{t}, J=7.6 \mathrm{~Hz}, 4 \mathrm{H}), 7.29-7.26(\mathrm{~m}, 2 \mathrm{H}), 6.54(\mathrm{dt}, J=16.8,10.4 \mathrm{~Hz}, 1 \mathrm{H}), 6.25(\mathrm{dd}, J$ $=15.2,10.4 \mathrm{~Hz}, 1 \mathrm{H}), 6.00(\mathrm{dd}, J=15.2,9.0 \mathrm{~Hz}, 1 \mathrm{H}), 5.33(\mathrm{~d}, J=16.8 \mathrm{~Hz}, 1 \mathrm{H}), 5.23(\mathrm{~d}, J=10.4 \mathrm{~Hz}, 1 \mathrm{H})$, $4.37(\mathrm{~d}, J=8.8 \mathrm{~Hz}, 1 \mathrm{H}), 3.70(\mathrm{~d}, J=13.6 \mathrm{~Hz}, 2 \mathrm{H}), 3.55(\mathrm{~d}, J=13.6 \mathrm{~Hz}, 2 \mathrm{H}) .{ }^{19} \mathrm{~F} \mathrm{NMR}\left(282 \mathrm{MHz}, \mathrm{CDCl}_{3}\right)$ $\delta$-62.44. ${ }^{13} \mathrm{C}$ NMR $\left(75 \mathrm{MHz}, \mathrm{CDCl}_{3}\right) \delta 143.1,139.7,136.4,136.3,131.6(\mathrm{q}, J=1.2 \mathrm{~Hz}), 130.6(\mathrm{q}, J=$ $32.0 \mathrm{~Hz}), 130.1,128.8,128.8,128.5,127.1,124.9(\mathrm{q}, J=3.8 \mathrm{~Hz}), 124.4(\mathrm{q}, J=272.1 \mathrm{~Hz}), 124.0(\mathrm{q}, J=$ $3.8 \mathrm{~Hz}), 118.0,64.2$, 54.0. HRMS (ESI) calculated for $\mathrm{C}_{26} \mathrm{H}_{25} \mathrm{~F}_{3} \mathrm{~N}[\mathrm{M}+\mathrm{H}]^{+}$: 408.1939, found: 408.1930.

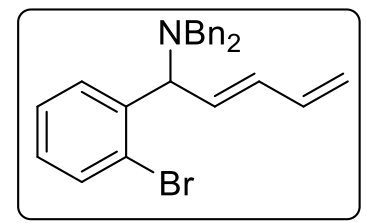

(2E)- $N, N$-Dibenzyl-5-o-bromophenyl-penta-1,3-dien-5-amine (3ap)

The title compound was prepared via general procedure A, purified by flash chromatography on silica gel eluting with in $\mathrm{CH}_{2} \mathrm{Cl}_{2} / \mathrm{PE}=1 / 3(\mathrm{Rf}=0.24)$, colorless oil, $36.5 \mathrm{mg}, 44 \%$ yield, ${ }^{1} \mathrm{H} \mathrm{NMR}(300 \mathrm{MHz}$, $\left.\mathrm{CDCl}_{3}\right) \delta 7.76(\mathrm{dd}, J=7.8,1.8 \mathrm{~Hz}, 1 \mathrm{H}), 7.53(\mathrm{dd}, J=8.1,1.2 \mathrm{~Hz}, 1 \mathrm{H}), 7.38-7.16(\mathrm{~m}, 11 \mathrm{H}), 7.08(\mathrm{td}, J=$ 
7.8, $1.8 \mathrm{~Hz}, 1 \mathrm{H}), 6.29(\mathrm{dd}, J=15.0,10.5 \mathrm{~Hz}, 1 \mathrm{H}), 6.14(\mathrm{dt}, J=16.8,10.2 \mathrm{~Hz}, 1 \mathrm{H}), 5.59(\mathrm{dd}, J=15.0,8.7$ $\mathrm{Hz}, 1 \mathrm{H}), 5.16(\mathrm{dd}, J=16.5,1.2 \mathrm{~Hz}, 1 \mathrm{H}), 5.01(\mathrm{dd}, J=9.9,1.5 \mathrm{~Hz}, 1 \mathrm{H}), 4.72$ (d, $J=8.7 \mathrm{~Hz}, 1 \mathrm{H}), 3.69$ (d, $J=14.1 \mathrm{~Hz}, 2 \mathrm{H}), 3.62(\mathrm{~d}, J=14.1 \mathrm{~Hz}, 2 \mathrm{H}) .{ }^{13} \mathrm{C} \mathrm{NMR}\left(75 \mathrm{MHz}, \mathrm{CDCl}_{3}\right) \delta 141.4,139.5,136.7,134.8$, 133.3, 132.6, 129.9, 129.3, 128.5, 128.2, 127.7, 126.8, 124.7, 117.3, 67.9, 54.8. HRMS (ESI) calculated for $\mathrm{C}_{25} \mathrm{H}_{25} \mathrm{BrN}[\mathrm{M}+\mathrm{H}]^{+}:$418.1170, found: 418.1163 .

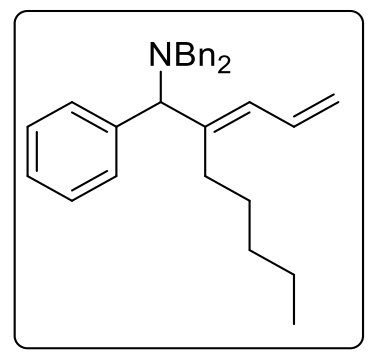

(2E)-N,N-Dibenzyl-4-pentyl-5-phenyl-penta-1,3-dien-5-amine (3aq)

The title compound was prepared via general procedure A, purified by flash chromatography on silica gel eluting with in $\mathrm{CH}_{2} \mathrm{Cl}_{2} / \mathrm{PE}=1 / 3(\mathrm{Rf}=0.34)$, colorless oil, $59.0 \mathrm{mg}, 72 \%$ yield, ${ }^{1} \mathrm{H} \mathrm{NMR}(400 \mathrm{MHz}$, $\left.\mathrm{CDCl}_{3}\right) \delta 7.38-7.29(\mathrm{~m}, 13 \mathrm{H}), 7.22(\mathrm{t}, J=7.2 \mathrm{~Hz}, 2 \mathrm{H}), 6.58(\mathrm{dt}, J=16.8,10.4 \mathrm{~Hz}, 1 \mathrm{H}), 6.26(\mathrm{~d}, J=11.2$ $\mathrm{Hz}, 1 \mathrm{H}), 5.14(\mathrm{dd}, J=16.8,1.6 \mathrm{~Hz}, 1 \mathrm{H}), 5.05(\mathrm{dd}, J=10.4,1.6 \mathrm{~Hz}, 1 \mathrm{H}), 4.30(\mathrm{~s}, 1 \mathrm{H}), 3.81(\mathrm{~d}, J=14.0$ $\mathrm{Hz}, 2 \mathrm{H}), 3.42$ (d, $J=14.0 \mathrm{~Hz}, 2 \mathrm{H}), 2.32-2.19$ (m, 1H), 2.11-1.95 (m, 1H), 1.18-1.09 (m, 5H), 0.95-0.88 $(\mathrm{m}, 1 \mathrm{H}), 0.79(\mathrm{t}, J=6.8 \mathrm{~Hz}, 3 \mathrm{H}) .{ }^{13} \mathrm{C} \mathrm{NMR}\left(100 \mathrm{MHz}, \mathrm{CDCl}_{3}\right) \delta 144.3,139.7,139.0,133.4,129.8,129.1$, 128.9, 128.3, 128.2, 127.2, 126.8, 116.6, 69.5, 54.3, 32.2, 29.3, 29.2, 22.5, 14.1. HRMS (ESI) calculated for $\mathrm{C}_{30} \mathrm{H}_{36} \mathrm{~N}[\mathrm{M}+\mathrm{H}]^{+}$: 410.2848, found: 410.2864 .

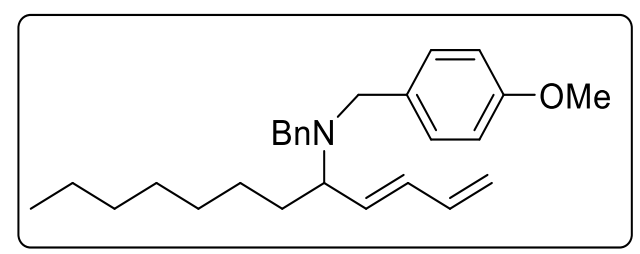

(3E)-N-benzyl- $N$-p-methoxylbenzyl-dodeca-1,3-dien-5-amine (3ba)

The title compound was prepared via general procedure A, purified by flash chromatography on silica gel eluting with in $\mathrm{CH}_{2} \mathrm{Cl}_{2} / \mathrm{PE}=1 / 3(\mathrm{Rf}=0.21)$, colorless oil, $65.3 \mathrm{mg}, 83 \%$ yield, ${ }^{1} \mathrm{H} \mathrm{NMR}(400 \mathrm{MHz}$, $\left.\mathrm{CDCl}_{3}\right) \delta 7.36(\mathrm{~d}, J=7.2 \mathrm{~Hz}, 2 \mathrm{H}), 7.32-7.24(\mathrm{~m}, 4 \mathrm{H}), 7.21(\mathrm{t}, J=7.2 \mathrm{~Hz}, 1 \mathrm{H}), 6.84(\mathrm{~d}, J=8.4 \mathrm{~Hz}, 2 \mathrm{H})$, 6.46-6.29 (m, 1H), 6.03 (dd, $J=15.2,10.4 \mathrm{~Hz}, 1 \mathrm{H}), 5.69$ (dd, $J=15.2,8.8 \mathrm{~Hz}, 1 \mathrm{H}), 5.19$ (d, $J=17.2 \mathrm{~Hz}$, 1H), $5.07(\mathrm{~d}, J=10.0 \mathrm{~Hz}, 1 \mathrm{H}), 3.79(\mathrm{~s}, 3 \mathrm{H}), 3.71(\mathrm{dd}, J=17.2,13.6 \mathrm{~Hz}, 2 \mathrm{H}), 3.31(\mathrm{dd}, J=17.2,13.6 \mathrm{~Hz}$, $2 \mathrm{H}), 3.02(\mathrm{q}, J=7.2 \mathrm{~Hz}, 1 \mathrm{H}), 1.73-1.66(\mathrm{~m}, 1 \mathrm{H}), 1.43-1.12(\mathrm{~m}, 11 \mathrm{H}), 0.87(\mathrm{t}, J=7.2 \mathrm{~Hz}, 3 \mathrm{H}) .{ }^{13} \mathrm{C} \mathrm{NMR}$ $\left(100 \mathrm{MHz}, \mathrm{CDCl}_{3}\right) \delta 158.5,140.8,137.0,133.7,133.4,132.6,129.9,128.8,128.3,126.7,116.3,113.6$, 59.7, 55.4, 53.6, 53.1, 32.5, 32.1, 29.7, 29.4, 26.6, 22.8, 14.3. HRMS (ESI) calculated for $\mathrm{C}_{27} \mathrm{H}_{38} \mathrm{NO}$ $[\mathrm{M}+\mathrm{H}]^{+}:$392.2953, found: 392.2950 . 


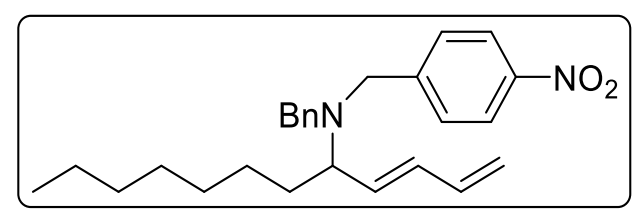

(3E)- $N$-Benzyl- $N$-p-nitrylbenzyl-dodeca-1,3-dien-5-amine (3ca)

The title compound was prepared via general procedure A, purified by flash chromatography on silica gel eluting with in EtOAc/PE $=1 / 10(\mathrm{Rf}=0.30)$, yellow oil, $73.1 \mathrm{mg}, 90 \%$ yield. ${ }^{1} \mathrm{H} \mathrm{NMR}\left(400 \mathrm{MHz}, \mathrm{CDCl}_{3}\right)$ $\delta 8.15(\mathrm{~d}, J=8.8 \mathrm{~Hz}, 2 \mathrm{H}), 7.52(\mathrm{~d}, J=8.8 \mathrm{~Hz}, 2 \mathrm{H}), 7.38-7.20(\mathrm{~m}, 5 \mathrm{H}), 6.39(\mathrm{dt}, J=17.2,10.4 \mathrm{~Hz}, 1 \mathrm{H})$, $6.03(\mathrm{dd}, J=15.2,10.4 \mathrm{~Hz}, 1 \mathrm{H}), 5.68(\mathrm{dd}, J=15.2,8.8 \mathrm{~Hz}, 1 \mathrm{H}), 5.21(\mathrm{~d}, J=17.2 \mathrm{~Hz}, 1 \mathrm{H}), 5.11(\mathrm{~d}, J=$ $10.4 \mathrm{~Hz}, 1 \mathrm{H}), 3.81(\mathrm{~d}, J=14.0 \mathrm{~Hz}, 1 \mathrm{H}), 3.79(\mathrm{dd}, J=14.0 \mathrm{~Hz}, 1 \mathrm{H}), 3.50$ (d, $J=14.8 \mathrm{~Hz}, 1 \mathrm{H}), 3.40$ (d, $J$ $=14.8 \mathrm{~Hz}, 1 \mathrm{H}), 2.98(\mathrm{q}, J=7.2 \mathrm{~Hz}, 1 \mathrm{H}), 1.76-1.61(\mathrm{~m}, 1 \mathrm{H}), 1.52-1.41(\mathrm{~m}, 1 \mathrm{H}), 1.38-1.11(\mathrm{~m}, 10 \mathrm{H}), 0.87$ $(\mathrm{t}, J=7.2 \mathrm{~Hz}, 3 \mathrm{H}) .{ }^{13} \mathrm{C} \mathrm{NMR}\left(100 \mathrm{MHz}, \mathrm{CDCl}_{3}\right) \delta 148.9,147.0,139.9,136.7,134.2,132.4,129.2,128.8$, $128.4,127.1,123.6,117.0,60.7,54.0,53.6,32.6,32.0,29.6,29.4,26.6,22.8,14.2$. HRMS (ESI) calculated for $\mathrm{C}_{26} \mathrm{H}_{35} \mathrm{~N}_{2} \mathrm{O}_{2}[\mathrm{M}+\mathrm{H}]^{+}:$407.2699, found: 407.2704.

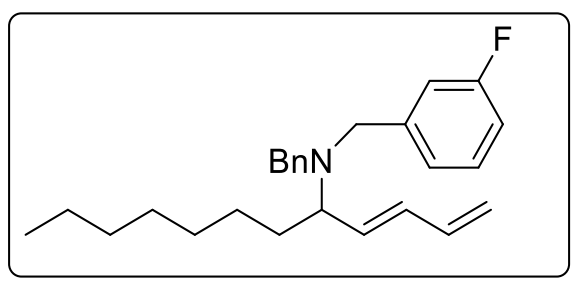

\section{(3E)- $N$-Benzyl- $N$-m-fluorobenzyldodeca-1,3-dien-5-amine (3da)}

The title compound was prepared via general procedure A, purified by flash chromatography on silica gel eluting with in $\mathrm{CH}_{2} \mathrm{Cl}_{2} / \mathrm{PE}=1 / 3(\mathrm{Rf}=0.30)$, yellow oil, $56.7 \mathrm{mg}, 70 \%$ yield. ${ }^{1} \mathrm{H} \mathrm{NMR}\left(300 \mathrm{MHz}, \mathrm{CDCl}_{3}\right)$ $\delta$ 7.42-7.19 (m, 6H), $7.12(\mathrm{~d}, J=7.6 \mathrm{~Hz}, 2 \mathrm{H}), 6.90(\mathrm{dd}, J=9.6,7.6 \mathrm{~Hz}, 1 \mathrm{H}), 6.38(\mathrm{dt}, J=17.2,10.4 \mathrm{~Hz}$, $1 \mathrm{H}), 6.03(\mathrm{dd}, J=15.2,10.4 \mathrm{~Hz}, 1 \mathrm{H}), 5.68$ (dd, $J=15.2,8.8 \mathrm{~Hz}, 1 \mathrm{H}), 5.20$ (d, $J=17.2 \mathrm{~Hz}, 1 \mathrm{H}), 5.08$ (d, $J=10.4 \mathrm{~Hz}, 1 \mathrm{H}), 3.79$ (d, $J=7.2 \mathrm{~Hz}, 1 \mathrm{H}), 3.75$ (d, $J=7.2 \mathrm{~Hz}, 1 \mathrm{H}), 3.37$ (d, $J=14.0 \mathrm{~Hz}, 1 \mathrm{H}), 3.36(\mathrm{~d}, J$ $=14.0 \mathrm{~Hz}, 1 \mathrm{H}), 3.02(\mathrm{q}, J=7.2 \mathrm{~Hz}, 1 \mathrm{H}), 1.74-1.61(\mathrm{~m}, 1 \mathrm{H}), 1.49-1.15(\mathrm{~m}, 11 \mathrm{H}), 0.87(\mathrm{t}, J=7.2 \mathrm{~Hz}, 3 \mathrm{H})$.

${ }^{19} \mathrm{~F} \mathrm{NMR}\left(282 \mathrm{MHz}, \mathrm{CDCl}_{3}\right) \delta-113.95 .{ }^{13} \mathrm{C} \mathrm{NMR}\left(75 \mathrm{MHz}, \mathrm{CDCl}_{3}\right) \delta 163.2(\mathrm{~d}, J=244.9 \mathrm{~Hz}), 143.6(\mathrm{~d}$, $J=7.0 \mathrm{~Hz}), 140.4,136.9,133.9,132.9,129.6(\mathrm{~d}, J=8.2 \mathrm{~Hz}), 128.8,128.4,126.9,124.2(\mathrm{~d}, J=2.6 \mathrm{~Hz})$, 116.6, 115.4 (d, $J=21.3 \mathrm{~Hz}), 113.7$ (d, $J=21.3 \mathrm{~Hz}), 60.1,53.9,53.5,32.5,32.0,29.6,29.4,26.6,22.8$, 14.3. HRMS (ESI) calculated for $\mathrm{C}_{26} \mathrm{H}_{35} \mathrm{FN}[\mathrm{M}+\mathrm{H}]^{+}: 380.2754$, found: 380.2755 .

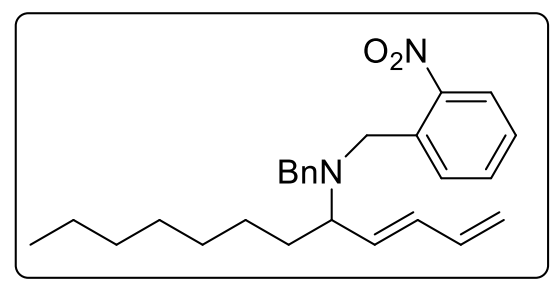

(3E)- $N$-benzyl- $N$-o-nitrylbenzyldodeca-1,3-dien-5-amine (3ea)

The title compound was prepared via general procedure A, purified by flash chromatography on silica gel 
eluting with in $\mathrm{CH}_{2} \mathrm{Cl}_{2} / \mathrm{PE}=1 / 3(\mathrm{Rf}=0.35)$, yellow oil, $73.9 \mathrm{mg}, 91 \%$ yield, ${ }^{1} \mathrm{H} \mathrm{NMR}\left(400 \mathrm{MHz}, \mathrm{CDCl}_{3}\right)$ $\delta 7.91(\mathrm{~d}, J=8.0 \mathrm{~Hz}, 1 \mathrm{H}), 7.82(\mathrm{dd}, J=8.0,0.8 \mathrm{~Hz}, 1 \mathrm{H}), 7.61-7.49(\mathrm{~m}, 1 \mathrm{H}), 7.39-7.20(\mathrm{~m}, 6 \mathrm{H}), 6.41(\mathrm{dt}$, $J=17.2,10.4 \mathrm{~Hz}, 1 \mathrm{H}), 6.04(\mathrm{dd}, J=15.2,10.4 \mathrm{~Hz}, 1 \mathrm{H}), 5.72(\mathrm{dd}, J=15.2,8.8 \mathrm{~Hz}, 1 \mathrm{H}), 5.23(\mathrm{~d}, J=16.8$ $\mathrm{Hz}, 1 \mathrm{H}), 5.12(\mathrm{~d}, J=10.4 \mathrm{~Hz}, 1 \mathrm{H}), 4.10(\mathrm{~d}, J=16.0 \mathrm{~Hz}, 1 \mathrm{H}), 3.80(\mathrm{~d}, J=16.0 \mathrm{~Hz}, 1 \mathrm{H}), 3.74(\mathrm{~d}, J=13.6$ $\mathrm{Hz}, 1 \mathrm{H}), 3.46(\mathrm{~d}, J=13.6 \mathrm{~Hz}, 1 \mathrm{H}), 3.03$ (q, $J=8.0 \mathrm{~Hz}, 1 \mathrm{H}), 1.77-1.67$ (m, 1H), 1.57-1.42 (m, 1H), 1.34$1.11(\mathrm{~m}, 10 \mathrm{H}), 0.91(\mathrm{t}, J=6.8 \mathrm{~Hz}, 3 \mathrm{H}) .{ }^{13} \mathrm{C} \mathrm{NMR}\left(100 \mathrm{MHz}, \mathrm{CDCl}_{3}\right) \delta 149.9,139.7,136.7,135.9,134.3$, 132.6, 132.4, 130.9, 128.8, 128.3, 127.5, 127.0, 124.2, 116.9, 61.4, 54.8, 50.7, 32.4, 31.9, 29.6, 29.3, 26.8, 22.8, 14.2. HRMS (ESI) calculated for $\mathrm{C}_{26} \mathrm{H}_{35} \mathrm{~N}_{2} \mathrm{O}_{2}[\mathrm{M}+\mathrm{H}]^{+}:$407.2699, found: 407.2701.

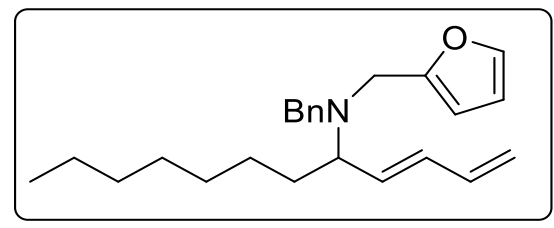

(3E) - N-Benzyl- $N$-2-furylmethyl-dodeca-1,3-dien-5-amine (3fa)

The title compound was prepared via general procedure A, purified by flash chromatography on silica gel eluting with in $\mathrm{CH}_{2} \mathrm{Cl}_{2} / \mathrm{PE}=1 / 3(\mathrm{Rf}=0.44)$, yellow oil, $73.9 \mathrm{mg}, 88 \%$ yield, ${ }^{1} \mathrm{H} \mathrm{NMR}\left(400 \mathrm{MHz}, \mathrm{CDCl}_{3}\right)$ $\delta$ 7.38-7.19 (m, 6H), 6.33-6.29 (m, 1H), $6.29(\mathrm{t}, J=3.2 \mathrm{~Hz}, 1 \mathrm{H}$, furyl-H), 6.17 (d, $J=3.2 \mathrm{~Hz}, 1 \mathrm{H}$, furylH), $6.08(\mathrm{dd}, J=15.2,10.4 \mathrm{~Hz}, 1 \mathrm{H}), 5.61(\mathrm{dd}, J=15.2,8.8 \mathrm{~Hz}, 1 \mathrm{H}), 5.18(\mathrm{~d}, J=16.8 \mathrm{~Hz}, 1 \mathrm{H}), 5.06(\mathrm{~d}, J$ $=10.4 \mathrm{~Hz}, 1 \mathrm{H}), 3.83(\mathrm{~d}, J=14.0 \mathrm{~Hz}, 1 \mathrm{H}), 3.70(\mathrm{~d}, J=14.8 \mathrm{~Hz}, 1 \mathrm{H}), 3.50(\mathrm{~d}, J=14.8 \mathrm{~Hz}, 1 \mathrm{H}), 3.44(\mathrm{~d}, J$ $=14.0 \mathrm{~Hz}, 1 \mathrm{H}), 3.08(\mathrm{q}, J=7.2 \mathrm{~Hz}, 1 \mathrm{H}), 1.76-1.55(\mathrm{~m}, 1 \mathrm{H}), 1.54-1.12(\mathrm{~m}, 11 \mathrm{H}), 0.87(\mathrm{t}, J=6.8 \mathrm{~Hz}, 3 \mathrm{H})$. ${ }^{13} \mathrm{C}$ NMR $\left(100 \mathrm{MHz}, \mathrm{CDCl}_{3}\right) \delta 154.1,141.7,140.5,137.0,133.7,133.5,128.7,128.3,126.8,116.4,110.2$, 107.8, 61.0, 54.0, 46.7, 32.5, 32.0, 29.6, 29.4, 26.5, 22.8, 14.3. HRMS (ESI) calculated for $\mathrm{C}_{24} \mathrm{H}_{34} \mathrm{NO}$ $[\mathrm{M}+\mathrm{H}]^{+}:$352.2640, found: 352.2630 .

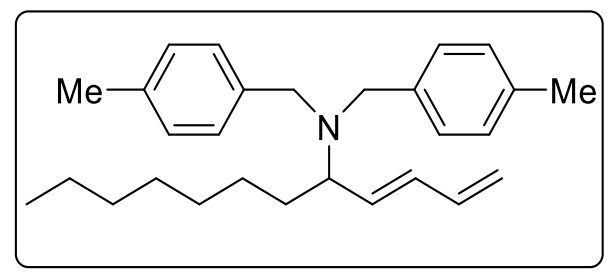

(3E)-N,N-Di-(p-methylbenzyl)-dodeca-1,3-dien-5-amine (3ga)

The title compound was prepared via general procedure A, purified by flash chromatography on silica gel eluting with in $\mathrm{CH}_{2} \mathrm{Cl}_{2} / \mathrm{PE}=1 / 3(\mathrm{Rf}=0.22)$, yellow oil, $59.3 \mathrm{mg}, 76 \%$ yield. ${ }^{1} \mathrm{H} \mathrm{NMR}\left(400 \mathrm{MHz}, \mathrm{CDCl}_{3}\right)$ $\delta 7.25(\mathrm{~d}, J=8.0 \mathrm{~Hz}, 4 \mathrm{H}), 7.10(\mathrm{~d}, J=8.0 \mathrm{~Hz}, 4 \mathrm{H}), 6.38(\mathrm{dt}, J=17.2,10.4 \mathrm{~Hz}, 1 \mathrm{H}), 6.03(\mathrm{dd}, J=15.2$, $10.4 \mathrm{~Hz}, 1 \mathrm{H}), 5.69$ (dd, $J=15.2,8.8 \mathrm{~Hz}, 1 \mathrm{H}), 5.18$ (d, $J=16.8 \mathrm{~Hz}, 1 \mathrm{H}), 5.06$ (d, $J=10.4 \mathrm{~Hz}, 1 \mathrm{H}), 3.73$ $(\mathrm{d}, J=13.6 \mathrm{~Hz}, 2 \mathrm{H}), 3.30(\mathrm{~d}, J=13.6 \mathrm{~Hz}, 2 \mathrm{H}), 3.03$ (q, $J=7.2 \mathrm{~Hz}, 1 \mathrm{H}), 2.32(\mathrm{~s}, 6 \mathrm{H}), 1.74-1.61(\mathrm{~m}, 1 \mathrm{H})$, 1.44-1.33 (m, 2H), 1.30-1.11 (m, 9H), $0.87(\mathrm{t}, J=7.2 \mathrm{~Hz}, 3 \mathrm{H}) .{ }^{13} \mathrm{C}$ NMR (100 MHz, $\left.\mathrm{CDCl}_{3}\right) \delta 137.7$, 137.1, 136.2, 133.6, 133.5, 128.9, 128.7, 116.2, 59.6, 53.4, 32.4, 32.1, 29.7, 29.4, 26.6, 22.8, 21.2, 14.3. HRMS (ESI) calculated for $\mathrm{C}_{28} \mathrm{H}_{40} \mathrm{~N}[\mathrm{M}+\mathrm{H}]^{+}:$390.3161, found: 390.3166 . 


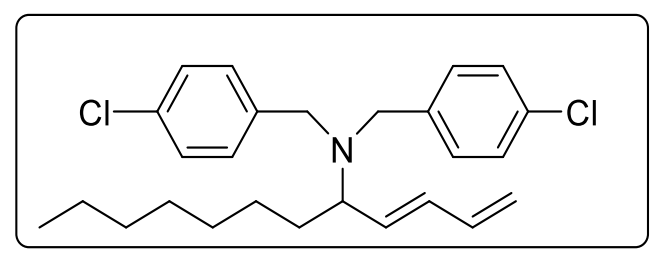

(3E)-N,N-di-p-chlorobenzyldodeca-1,3-dien-5-amine (3ha)

The title compound was prepared via general procedure A, purified by flash chromatography on silica gel eluting with in $\mathrm{CH}_{2} \mathrm{Cl}_{2} / \mathrm{PE}=1 / 3(\mathrm{Rf}=0.25)$, yellow oil, $72.1 \mathrm{mg}, 84 \%$ yield, ${ }^{1} \mathrm{H} \mathrm{NMR}\left(300 \mathrm{MHz}, \mathrm{CDCl}_{3}\right)$ $\delta 7.27$ (br, 8H, Ar-H), 6.37 (dt, $J=17.1,10.2 \mathrm{~Hz}, 1 \mathrm{H}), 6.01$ (dd, $J=15.3,10.5 \mathrm{~Hz}, 1 \mathrm{H}), 5.65$ (dd, $J=$ 15.3, 9.0 Hz, 1H), $5.20(\mathrm{dd}, J=17.1,1.5 \mathrm{~Hz}, 1 \mathrm{H}), 5.09$ (dd, $J=10.2,1.5 \mathrm{~Hz}, 1 \mathrm{H}), 3.70(\mathrm{~d}, J=13.8 \mathrm{~Hz}$, 2H), $3.31(\mathrm{~d}, J=13.8 \mathrm{~Hz}, 2 \mathrm{H}), 2.96(\mathrm{q}, J=7.2 \mathrm{~Hz}, 1 \mathrm{H}), 1.69-1.60(\mathrm{~m}, 1 \mathrm{H}), 1.46-1.08(\mathrm{~m}, 11 \mathrm{H}), 0.87$ (t, $J=6.9 \mathrm{~Hz}, 3 \mathrm{H}) .{ }^{13} \mathrm{C} \mathrm{NMR}\left(75 \mathrm{MHz}, \mathrm{CDCl}_{3}\right) \delta 138.9,136.8,134.1,132.6,132.5,130.1,128.5,116.8$, 60.1, 53.2, 32.5, 32.0, 29.6, 29.4, 26.6, 22.8, 14.3. HRMS (ESI) calculated for $\mathrm{C}_{26} \mathrm{H}_{34} \mathrm{Cl}_{2} \mathrm{~N}[\mathrm{M}+\mathrm{H}]^{+}$: 430.2068, found: 430.2065 .

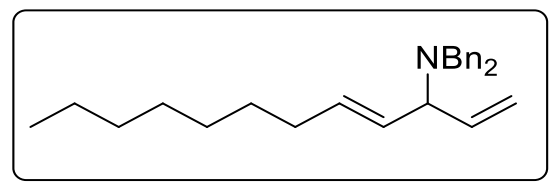

(4E)- $N, N$-Dibenzyl-dodeca-1,4-dien-3-amine (4aa)

The title compound was prepared via general procedure B, purified by flash chromatography on silica gel eluting with in $\mathrm{CH}_{2} \mathrm{Cl}_{2} / \mathrm{PE}=1 / 3(\mathrm{Rf}=0.30)$, yellow oil, $52.1 \mathrm{mg}, 72 \%$ yield, ${ }^{1} \mathrm{H} \mathrm{NMR}\left(400 \mathrm{MHz}, \mathrm{CDCl}_{3}\right)$ $\delta 7.44(\mathrm{~d}, J=7.2 \mathrm{~Hz}, 4 \mathrm{H}), 7.34(\mathrm{t}, J=7.2 \mathrm{~Hz}, 4 \mathrm{H}), 7.25$ (t, $J=7.2 \mathrm{~Hz}, 2 \mathrm{H}), 6.14-5.88(\mathrm{~m}, 1 \mathrm{H}), 5.62-5.44$ $(\mathrm{m}, 2 \mathrm{H}), 5.27(\mathrm{~d}, J=10.4 \mathrm{~Hz}, 1 \mathrm{H}), 5.16(\mathrm{~d}, J=17.2 \mathrm{~Hz}, 1 \mathrm{H}), 3.72-3.64(\mathrm{~m}, 3 \mathrm{H}), 3.60(\mathrm{~d}, J=14.0 \mathrm{~Hz}$, $2 \mathrm{H}), 2.14(\mathrm{q}, J=6.8 \mathrm{~Hz}, 2 \mathrm{H}), 1.48-1.26(\mathrm{~m}, 10 \mathrm{H}), 0.93(\mathrm{t}, J=6.8 \mathrm{~Hz}, 3 \mathrm{H}) .{ }^{13} \mathrm{C} \mathrm{NMR}\left(100 \mathrm{MHz}, \mathrm{CDCl}_{3}\right)$ $\delta 140.7,138.8,135.1,128.7,128.3,128.0,126.8,117.2,62.8,53.8,32.8,32.0,29.6,29.4,29.3,22.8,14.3$. HRMS (ESI) calculated for $\mathrm{C}_{26} \mathrm{H}_{36} \mathrm{~N}[\mathrm{M}+\mathrm{H}]^{+}: 362.2848$, found: 362.2838 .

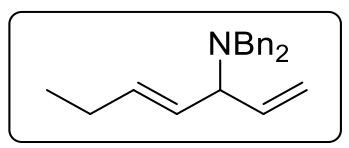

(4E)-N,N-Dibenzyl-hepta-1,4-dien-3-amine (4ab)

The title compound was prepared via general procedure B, purified by flash chromatography on silica gel eluting with in $\mathrm{CH}_{2} \mathrm{Cl}_{2} / \mathrm{PE}=1 / 3(\mathrm{Rf}=0.22)$, colorless oil, $47.1 \mathrm{mg}, 81 \%$ yield, ${ }^{1} \mathrm{H} \mathrm{NMR}(400 \mathrm{MHz}$, $\left.\mathrm{CDCl}_{3}\right) \delta 7.38(\mathrm{~d}, J=7.2 \mathrm{~Hz}, 4 \mathrm{H}), 7.29$ (t, $\left.J=7.2 \mathrm{~Hz}, 4 \mathrm{H}\right), 7.20$ (t, $\left.J=7.2 \mathrm{~Hz}, 2 \mathrm{H}\right), 6.06-5.83(\mathrm{~m}, 1 \mathrm{H})$, 5.62-5.43 (m, 2H), $5.23(\mathrm{dt}, J=10.4,1.6 \mathrm{~Hz}, 1 \mathrm{H}), 5.10(\mathrm{dt}, J=17.4,1.6 \mathrm{~Hz}, 1 \mathrm{H}), 3.67-3.59(\mathrm{~m}, 3 \mathrm{H}), 3.56$ $(\mathrm{d}, J=13.6 \mathrm{~Hz}, 2 \mathrm{H}), 2.19-2.00(\mathrm{~m}, 2 \mathrm{H}), 1.02(\mathrm{t}, J=7.5 \mathrm{~Hz}, 3 \mathrm{H}) .{ }^{13} \mathrm{C} \mathrm{NMR}\left(100 \mathrm{MHz}, \mathrm{CDCl}_{3}\right) \delta 140.7$, 138.6, 136.4, 128.7, 128.3, 127.2, 126.8, 117.3, 62.8, 53.8, 25.9, 14.1. HRMS (ESI) calculated for $\mathrm{C}_{21} \mathrm{H}_{26} \mathrm{~N}[\mathrm{M}+\mathrm{H}]^{+}:$292.2065, found: 292.2062. 


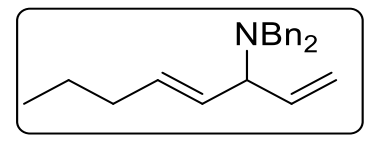

(4E)- $N, N$-Dibenzyl-octa-1,4-dien-3-amine (4ac)

The title compound was prepared via general procedure B, purified by flash chromatography on silica gel eluting with in $\mathrm{CH}_{2} \mathrm{Cl}_{2} / \mathrm{PE}=1 / 3(\mathrm{Rf}=0.24)$, colorless oil, $49.1 \mathrm{mg}, 80 \%$ yield, ${ }^{1} \mathrm{H} \mathrm{NMR}(400 \mathrm{MHz}$, $\left.\mathrm{CDCl}_{3}\right) \delta 7.42(\mathrm{~d}, J=7.2 \mathrm{~Hz}, 4 \mathrm{H}), 7.33(\mathrm{t}, J=7.2 \mathrm{~Hz}, 4 \mathrm{H}), 7.24(\mathrm{t}, J=7.2 \mathrm{~Hz}, 2 \mathrm{H}), 5.99$ (ddd, $J=17.2$, $10.4,6.0 \mathrm{~Hz}, 1 \mathrm{H}), 5.62-5.42(\mathrm{~m}, 2 \mathrm{H}), 5.26(\mathrm{dt}, J=10.4,1.6 \mathrm{~Hz}, 1 \mathrm{H}), 5.15(\mathrm{dt}, J=17.4,1.7 \mathrm{~Hz}, 1 \mathrm{H}), 3.73-$ $3.63(\mathrm{~m}, 3 \mathrm{H}), 3.60(\mathrm{~d}, J=14.0 \mathrm{~Hz}, 2 \mathrm{H}), 2.14-2.09(\mathrm{~m}, 2 \mathrm{H}), 1.46(\mathrm{dt}, J=14.8,7.2 \mathrm{~Hz}, 2 \mathrm{H}), 0.96(\mathrm{t}, J=$ $7.2 \mathrm{~Hz}, 3 \mathrm{H}) .{ }^{13} \mathrm{C} \mathrm{NMR}\left(100 \mathrm{MHz}, \mathrm{CDCl}_{3}\right) \delta 140.7,138.8,134.8,128.7,128.3,128.2,126.8,117.2,62.8$, 53.8, 34.9, 22.8, 13.9. HRMS (ESI) calculated for $\mathrm{C}_{22} \mathrm{H}_{28} \mathrm{~N}[\mathrm{M}+\mathrm{H}]^{+}: 306.2222$, found: 306.2220.

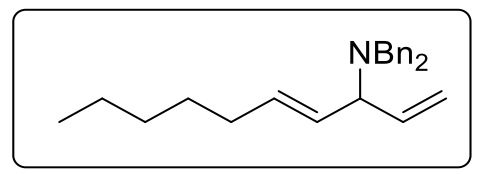

\section{(4E)- $N, N$-Dibenzyl-dodeca-1,4-dien-3-amine (4ad)}

The title compound was prepared via general procedure B, purified by flash chromatography on silica gel eluting with in $\mathrm{CH}_{2} \mathrm{Cl}_{2} / \mathrm{PE}=1 / 3(\mathrm{Rf}=0.28)$, colorless oil, $48.2 \mathrm{mg}, 72 \%$ yield, ${ }^{1} \mathrm{H} \mathrm{NMR}(400 \mathrm{MHz}$, $\left.\mathrm{CDCl}_{3}\right) \delta 7.41(\mathrm{~d}, J=7.2 \mathrm{~Hz}, 4 \mathrm{H}), 7.32$ (t, $\left.J=7.2 \mathrm{~Hz}, 4 \mathrm{H}\right), 7.23$ (t, $\left.J=7.2 \mathrm{~Hz}, 2 \mathrm{H}\right), 6.08-5.89$ (m, 1H), 5.59-5.44 (m, 2H), 5.30-5.22 (m, 1H), 5.17-5.07 (m, 1H), 3.71-3.62 (m, 3H), $3.58(\mathrm{~d}, J=14.0 \mathrm{~Hz}, 2 \mathrm{H})$, 2.15-2.03 (m, 2H), 1.48-1.24 (m, 6H), $0.92(\mathrm{t}, J=6.9 \mathrm{~Hz}, 3 \mathrm{H}) .{ }^{13} \mathrm{C}$ NMR $\left(100 \mathrm{MHz}, \mathrm{CDCl}_{3}\right) \delta 140.7$, $138.8,135.1$, 128.7, 128.3, 128.0, 126.8, 117.2, 62.8, 53.8, 32.8, 31.6, 29.3, 22.7, 14.2. HRMS (ESI) calculated for $\mathrm{C}_{24} \mathrm{H}_{32} \mathrm{~N}[\mathrm{M}+\mathrm{H}]^{+}: 334.2535$, found: 334.2543 .

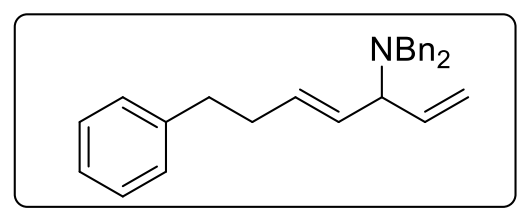

\section{(4E)- $N, N$-Dibenzyl-7-phenyl-hepta-1,4-dien-3-amine (4ae)}

The title compound was prepared via general procedure B, purified by flash chromatography on silica gel eluting with in $\mathrm{CH}_{2} \mathrm{Cl}_{2} / \mathrm{PE}=1 / 3(\mathrm{Rf}=0.22)$, colorless oil, $45.5 \mathrm{mg}, 62 \%$ yield, ${ }^{1} \mathrm{H} \mathrm{NMR}(300 \mathrm{MHz}$, $\left.\mathrm{CDCl}_{3}\right) \delta 7.41-6.99(\mathrm{~m}, 15 \mathrm{H}), 5.91(\mathrm{ddd}, J=17.4,10.5,6.0 \mathrm{~Hz}, 1 \mathrm{H}), 5.52-5.49(\mathrm{~m}, 2 \mathrm{H}), 5.20(\mathrm{dt}, J=10.5$, $1.8 \mathrm{~Hz}, 1 \mathrm{H}), 5.04$ (dt, $J=17.4,1.8 \mathrm{~Hz}, 1 \mathrm{H}), 3.61-3.54$ (m, 3H, -CH-, Ph-CH2-), 3.45 (d, J=13.8 Hz, 2H), $2.73(\mathrm{t}, J=7.5 \mathrm{~Hz}, 2 \mathrm{H}), 2.54-2.27(\mathrm{~m}, 2 \mathrm{H}) .{ }^{13} \mathrm{C} \mathrm{NMR}\left(75 \mathrm{MHz}, \mathrm{CDCl}_{3}\right) \delta 141.8,140.6,138.6,133.7$, 129.0, 128.7, 128.7, 128.4, 128.3, 126.8, 125.9, 117.3, 62.7, 53.7, 36.0, 34.5. HRMS (ESI) calculated for $\mathrm{C}_{27} \mathrm{H}_{30} \mathrm{~N}[\mathrm{M}+\mathrm{H}]^{+}: 368.2378$, found: 368.2374 .

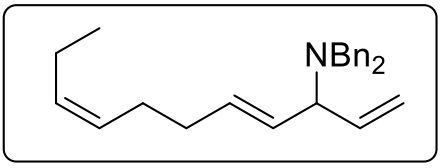


The title compound was prepared via general procedure B, purified by flash chromatography on silica gel eluting with in $\mathrm{CH}_{2} \mathrm{Cl}_{2} / \mathrm{PE}=1 / 3(\mathrm{Rf}=0.24)$, colorless oil, $48.8 \mathrm{mg}, 71 \%$ yield, ${ }^{1} \mathrm{H} \mathrm{NMR}(300 \mathrm{MHz}$, $\left.\mathrm{CDCl}_{3}\right) \delta$ 7.41-7.36 (m, 4H), 7.32-7.26 (m, 4H), 7.24-7.17 (m, 2H), 5.94 (ddd, J=17.1, 10.2, 6.0 Hz, 1H), 5.56-5.41 (m, 2H), 5.42-5.31 (m, 2H), $5.23(\mathrm{dt}, J=10.2,1.8 \mathrm{~Hz}, 1 \mathrm{H}), 5.11(\mathrm{dt}, J=17.1,1.8 \mathrm{~Hz}, 1 \mathrm{H}), 3.64$ $(\mathrm{t}, J=5.4 \mathrm{~Hz}, 1 \mathrm{H}), 3.59-3.54(\mathrm{~m}, 4 \mathrm{H}), 2.15-2.13(\mathrm{~m}, 4 \mathrm{H}), 2.09-1.98(\mathrm{~m}, 2 \mathrm{H}), 0.95(\mathrm{t}, J=7.5 \mathrm{~Hz}, 3 \mathrm{H}) .{ }^{13} \mathrm{C}$ NMR $\left(75 \mathrm{MHz}, \mathrm{CDCl}_{3}\right) \delta 140.6,138.5,134.2,132.2,128.72,128.67,128.5,128.3,126.8,117.4,62.8$, 53.8, 32.9, 27.2, 20.8, 14.5. HRMS (ESI) calculated for $\mathrm{C}_{25} \mathrm{H}_{32} \mathrm{~N}[\mathrm{M}+\mathrm{H}]^{+}:$346.2535, found: 346.2531 .

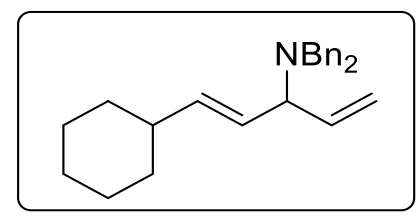

(4E)- $N, N$-Dibenzyl-1-cyclohexyl-1,4-dien-3-amine (4ag)

The title compound was prepared via general procedure B, purified by flash chromatography on silica gel eluting with in $\mathrm{CH}_{2} \mathrm{Cl}_{2} / \mathrm{PE}=1 / 3(\mathrm{Rf}=0.25)$, colorless oil, $46.7 \mathrm{mg}, 68 \%$ yield, ${ }^{1} \mathrm{H} \mathrm{NMR}(400 \mathrm{MHz}$, $\left.\mathrm{CDCl}_{3}\right) \delta 7.38(\mathrm{~d}, J=7.2 \mathrm{~Hz}, 4 \mathrm{H}), 7.29(\mathrm{t}, J=7.2 \mathrm{~Hz}, 4 \mathrm{H}), 7.20(\mathrm{t}, J=7.2 \mathrm{~Hz}, 2 \mathrm{H}), 5.94(\mathrm{ddd}, J=17.2$, $10.4,6.0 \mathrm{~Hz}, 1 \mathrm{H}), 5.55-5.35(\mathrm{~m}, 2 \mathrm{H}), 5.22$ (dt, $J=10.4,1.6 \mathrm{~Hz}, 1 \mathrm{H}), 5.10$ (dt, $J=17.2,1.6 \mathrm{~Hz}, 1 \mathrm{H}), 3.68$ $3.58(\mathrm{~m}, 1 \mathrm{H}), 3.63(\mathrm{~d}, J=14.0 \mathrm{~Hz}, 2 \mathrm{H}), 3.54$ (d, $J=14.0 \mathrm{~Hz}, 2 \mathrm{H}), 2.07-1.94(\mathrm{~m}, 1 \mathrm{H}), 1.82-1.59$ (m, 5H), 1.35-1.01 (m, 5H). ${ }^{13} \mathrm{C} \mathrm{NMR}\left(100 \mathrm{MHz}, \mathrm{CDCl}_{3}\right) \delta 141.0,140.7,138.9,128.7,128.3,126.8,125.2,117.2$, 62.7, 53.8, 41.0, 33.39, 33.37, 26.33, 26.2(2C). HRMS (ESI) calculated for $\mathrm{C}_{25} \mathrm{H}_{32} \mathrm{~N}[\mathrm{M}+\mathrm{H}]^{+}: 346.2535$, found: 346.2532 .

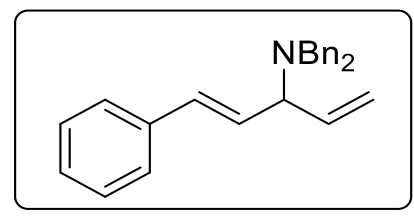

(4E)- $N, N$-Dibenzyl-1-phenyl-penta-1,4-dien-3-amine (4ah)

The title compound was prepared via general procedure $\mathrm{B}$, purified by flash chromatography on silica gel eluting with in $\mathrm{CH}_{2} \mathrm{Cl}_{2} / \mathrm{PE}=1 / 3(\mathrm{Rf}=0.25)$, colorless oil, $52.9 \mathrm{mg}, 78 \%$ yield, ${ }^{1} \mathrm{H} \mathrm{NMR}(400 \mathrm{MHz}$, $\left.\mathrm{CDCl}_{3}\right) \delta 7.40(\mathrm{t}, J=7.2 \mathrm{~Hz}, 6 \mathrm{H}), 7.34-7.29(\mathrm{~m}, 6 \mathrm{H}), 7.26-7.17(\mathrm{~m}, 3 \mathrm{H}), 6.45(\mathrm{~d}, J=16.0 \mathrm{~Hz}, 1 \mathrm{H}), 6.33$ $(\mathrm{dd}, J=16.0,6.4 \mathrm{~Hz}, 1 \mathrm{H}), 6.13-5.92(\mathrm{~m}, 1 \mathrm{H}), 5.38-5.27(\mathrm{~m}, 1 \mathrm{H}), 5.18(\mathrm{dt}, J=17.2,1.6 \mathrm{~Hz}, 1 \mathrm{H}), 3.87(\mathrm{t}$, $J=6.4 \mathrm{~Hz}, 1 \mathrm{H}), 3.72-3.53(\mathrm{~m}, 4 \mathrm{H}) .{ }^{13} \mathrm{C} \mathrm{NMR}\left(100 \mathrm{MHz}, \mathrm{CDCl}_{3}\right) \delta 140.4,137.8,137.2,133.2,129.0$, 128.7, 128.7, 128.4, 127.6, 126.9, 126.5, 118.2, 63.2, 54.0. HRMS (ESI) calculated for $\mathrm{C}_{25} \mathrm{H}_{26} \mathrm{~N}[\mathrm{M}+\mathrm{H}]^{+}$: 340.2065, found: 340.2053 .

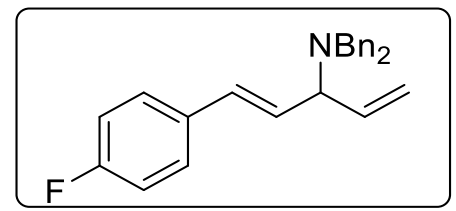




\section{(4E)- $N, N$-Dibenzyl-1-p-fluorophenyl-penta-1,4-dien-3-amine (4ai)}

The title compound was prepared via general procedure B, purified by flash chromatography on silica gel eluting with in $\mathrm{CH}_{2} \mathrm{Cl}_{2} / \mathrm{PE}=1 / 3(\mathrm{Rf}=0.25)$, yellow oil, $52.8 \mathrm{mg}, 74 \%$ yield, ${ }^{1} \mathrm{H} \mathrm{NMR}\left(300 \mathrm{MHz}, \mathrm{CDCl}_{3}\right)$ $\delta$ 7.48-7.17 (m, 12H), 7.03-6.88 (m, 2H), $6.40(\mathrm{~d}, J=16.0 \mathrm{~Hz}, 1 \mathrm{H}), 6.23(\mathrm{dd}, J=16.0,6.4 \mathrm{~Hz}, 1 \mathrm{H}), 6.02$ (ddd, $J=17.2,10.4,6.4 \mathrm{~Hz}, 1 \mathrm{H}), 5.32(\mathrm{~d}, J=10.4 \mathrm{~Hz}, 1 \mathrm{H}), 5.17$ (d, $J=17.2 \mathrm{~Hz}, 1 \mathrm{H}), 3.85$ (t, $J=6.4 \mathrm{~Hz}$, $1 \mathrm{H}), 3.66(\mathrm{~s}, 4 \mathrm{H}, \mathrm{Bn}) .{ }^{19} \mathrm{~F} \mathrm{NMR}\left(282 \mathrm{MHz}, \mathrm{CDCl}_{3}\right) \delta-114.68 .{ }^{13} \mathrm{C} \mathrm{NMR}\left(75 \mathrm{MHz}, \mathrm{CDCl}_{3}\right) \delta 162.4(\mathrm{~d}, J$ $=246.5 \mathrm{~Hz}), 140.3,137.5,133.4(\mathrm{~d}, J=3.3 \mathrm{~Hz}), 131.8,129.1(\mathrm{~d}, J=2.1 \mathrm{~Hz}), 128.7,128.4,128.9(\mathrm{~d}, J=$ $7.9 \mathrm{~Hz}), 126.9,118.3,115.6(\mathrm{~d}, J=21.5 \mathrm{~Hz}), 63.3,54.1$. HRMS (ESI) calculated for $\mathrm{C}_{25} \mathrm{H}_{25} \mathrm{FN}[\mathrm{M}+\mathrm{H}]^{+}$: 358.1971, found: 358.1975 .

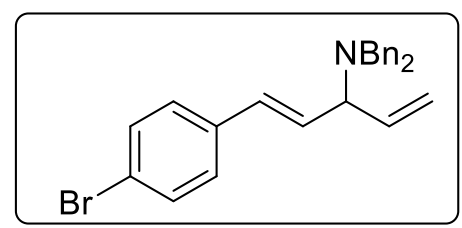

\section{(4E)- $N, N$-Dibenzyl-1-p-bromophenyl-penta-1,4-dien-3-amine (4aj)}

The title compound was prepared via general procedure B, purified by flash chromatography on silica gel eluting with in $\mathrm{CH}_{2} \mathrm{Cl}_{2} / \mathrm{PE}=1 / 3(\mathrm{Rf}=0.25)$, yellow oil, $63.1 \mathrm{mg}, 75 \%$ yield, ${ }^{1} \mathrm{H} \mathrm{NMR}\left(400 \mathrm{MHz}, \mathrm{CDCl}_{3}\right)$ $\delta$ 7.48-7.43 (m, 6H), $7.35(\mathrm{t}, J=7.5 \mathrm{~Hz}, 4 \mathrm{H}), 7.28-7.24(\mathrm{~m}, 4 \mathrm{H}), 6.49-6.27(\mathrm{~m}, 2 \mathrm{H}), 6.05$ (ddd, $J=17.1$, 10.4, $6.5 \mathrm{~Hz}, 1 \mathrm{H}), 5.37$ (d, $J=10.4 \mathrm{~Hz}, 1 \mathrm{H}), 5.20$ (d, $J=17.4 \mathrm{~Hz}, 1 \mathrm{H}), 3.88$ (t, $J=6.4 \mathrm{~Hz}, 1 \mathrm{H}), 3.70$ (s, $4 \mathrm{H}) .{ }^{13} \mathrm{C}$ NMR $\left(75 \mathrm{MHz}, \mathrm{CDCl}_{3}\right) \delta 140.3,137.3,136.2,131.8(2 \mathrm{C}), 130.4,128.7,128.4,128.0,126.9$, 121.3, 118.4, 63.3, 54.1. HRMS (ESI) calculated for $\mathrm{C}_{25} \mathrm{H}_{25} \mathrm{BrN}[\mathrm{M}+\mathrm{H}]^{+}$: 418.1170, found: 418.1163 .

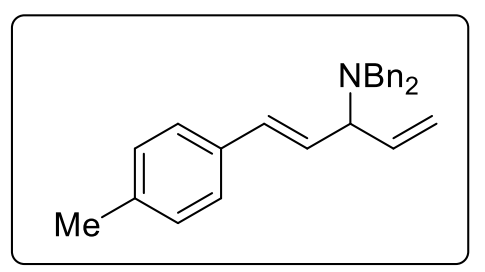

(4E)- $N, N$-Dibenzyl-1-p-methylphenyl-penta-1,4-dien-3-amine (4ak)

The title compound was prepared via general procedure B, purified by flash chromatography on silica gel eluting with in $\mathrm{CH}_{2} \mathrm{Cl}_{2} / \mathrm{PE}=1 / 3(\mathrm{Rf}=0.25)$, yellow oil, $51.0 \mathrm{mg}, 72 \%$ yield, ${ }^{1} \mathrm{H} \mathrm{NMR}\left(400 \mathrm{MHz}, \mathrm{CDCl}_{3}\right)$ $\delta 7.41(\mathrm{~d}, J=7.2 \mathrm{~Hz}, 4 \mathrm{H}), 7.32-7.28(\mathrm{~m}, 6 \mathrm{H}), 7.23-7.19(\mathrm{~m}, 2 \mathrm{H}), 7.12(\mathrm{~d}, J=8.0 \mathrm{~Hz}, 2 \mathrm{H}), 6.41(\mathrm{~d}, J=$ $16.0 \mathrm{~Hz}, 1 \mathrm{H}), 6.27$ (dd, $J=16.0,6.8 \mathrm{~Hz}, 1 \mathrm{H}), 6.03(\mathrm{ddd}, J=16.8,10.4,6.4 \mathrm{~Hz}, 1 \mathrm{H}), 5.30$ (dt, $J=17.2$, $1.6 \mathrm{~Hz}, 1 \mathrm{H}), 5.17(\mathrm{dt}, J=17.2,1.6 \mathrm{~Hz}, 1 \mathrm{H}), 3.85$ (t, $J=6.4 \mathrm{~Hz}, 1 \mathrm{H}), 3.68$ (d, $J=14.0 \mathrm{~Hz}, 2 \mathrm{H}), 3.64(\mathrm{~d}, J$ $=14.0 \mathrm{~Hz}, 2 \mathrm{H}), 2.33(\mathrm{~s}, 3 \mathrm{H}) \cdot{ }^{13} \mathrm{C} \mathrm{NMR}\left(100 \mathrm{MHz}, \mathrm{CDCl}_{3}\right) \delta 140.4,138.0,137.4,134.5,133.1,129.4$, 128.7, 128.3, 127.9, 126.9, 126.4, 118.0, 63.2, 54.0, 21.3. HRMS (ESI) calculated for $\mathrm{C}_{26} \mathrm{H}_{28} \mathrm{~N}[\mathrm{M}+\mathrm{H}]^{+}$: 354.2222, found: 354.2224 . 


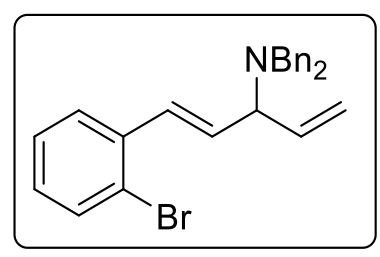

(4E)- $N, N$-Dibenzyl-1-o-bromophenyl-penta-2,4-dien-3-amine (4al)

The title compound was prepared via general procedure B, purified by flash chromatography on silica gel eluting with in $\mathrm{CH}_{2} \mathrm{Cl}_{2} / \mathrm{PE}=1 / 3(\mathrm{Rf}=0.20)$, colorless oil, $68.4 \mathrm{mg}, 82 \%$ yield, ${ }^{1} \mathrm{H} \mathrm{NMR}(400 \mathrm{MHz}$, $\left.\mathrm{CDCl}_{3}\right) \delta 7.53(\mathrm{ddd}, J=13.6,8.0,1.2 \mathrm{~Hz}, 2 \mathrm{H}), 7.44(\mathrm{~d}, J=7.2 \mathrm{~Hz}, 4 \mathrm{H}), 7.35-7.22(\mathrm{~m}, 7 \mathrm{H}), 7.10(\mathrm{td}, J=$ 8.0, 1.6 Hz, 1H), $6.81(\mathrm{~d}, J=16.0 \mathrm{~Hz}, 1 \mathrm{H}), 6.24(\mathrm{dd}, J=16.0,6.8 \mathrm{~Hz}, 1 \mathrm{H}), 6.11-5.96(\mathrm{~m}, 1 \mathrm{H}), 5.34(\mathrm{dt}$, $J=10.4,1.6 \mathrm{~Hz}, 1 \mathrm{H}), 5.21(\mathrm{dt}, J=17.2,1.6 \mathrm{~Hz}, 1 \mathrm{H}), 3.99-3.81(\mathrm{~m}, 1 \mathrm{H}), 3.70(\mathrm{~d}, J=14.0 \mathrm{~Hz}, 2 \mathrm{H}), 3.65$ $(\mathrm{d}, J=14.0 \mathrm{~Hz}, 2 \mathrm{H}) .{ }^{13} \mathrm{C} \mathrm{NMR}\left(100 \mathrm{MHz}, \mathrm{CDCl}_{3}\right) \delta 140.2,137.6,137.2,132.9,132.14,132.01,128.76$, $128.69,128.28,127.5,127.2,126.8,123.6,118.2,62.9,53.9$. HRMS (ESI) calculated for $\mathrm{C}_{25} \mathrm{H}_{25} \mathrm{BrN}$ $[\mathrm{M}+\mathrm{H}]^{+}:$418.1170, found: 418.1163 .

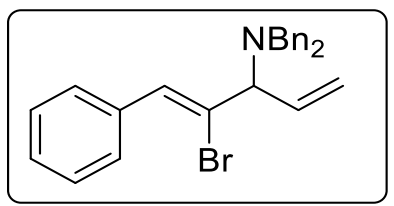

\section{(4E)- $N, N$-Dibenzyl-1-phenyl-2-bromo-penta-1,4-dien-3-amine (4am)}

The title compound was prepared via general procedure B, purified by flash chromatography on silica gel eluting with in $\mathrm{CH}_{2} \mathrm{Cl}_{2} / \mathrm{PE}=1 / 3(\mathrm{Rf}=0.22)$, yellow oil, $66.9 \mathrm{mg}, 80 \%$ yield, ${ }^{1} \mathrm{H} \mathrm{NMR}\left(300 \mathrm{MHz}, \mathrm{CDCl}_{3}\right)$ $\delta$ 7.61-7.54 (m, 2H), $7.47(\mathrm{~d}, J=7.1 \mathrm{~Hz}, 4 \mathrm{H}), 7.37-7.20(\mathrm{~m}, 9 \mathrm{H}), 7.00(\mathrm{~s}, 1 \mathrm{H}), 6.08(\mathrm{ddd}, J=16.8,10.4$, $8.0 \mathrm{~Hz}, 1 \mathrm{H}), 5.45$ (dd, $J=10.4,1.2 \mathrm{~Hz}, 1 \mathrm{H}), 5.24$ (dd, $J=16.8,0.8 \mathrm{~Hz}, 1 \mathrm{H}), 4.00(\mathrm{~d}, J=8.0 \mathrm{~Hz}, 1 \mathrm{H})$, $3.89(\mathrm{~d}, J=14.0 \mathrm{~Hz}, 2 \mathrm{H}), 3.59$ (d, $J=14.0 \mathrm{~Hz}, 2 \mathrm{H}) .{ }^{13} \mathrm{C} \mathrm{NMR}\left(75 \mathrm{MHz}, \mathrm{CDCl}_{3}\right) \delta 139.6,136.0,134.0$, $130.5,129.3,129.0,128.8,128.4,128.2,128.0,127.1,120.2,69.9,53.8$. HRMS (ESI) calculated for $\mathrm{C}_{25} \mathrm{H}_{25} \mathrm{BrN}[\mathrm{M}+\mathrm{H}]^{+}: 418.1170$, found: 418.1163 .

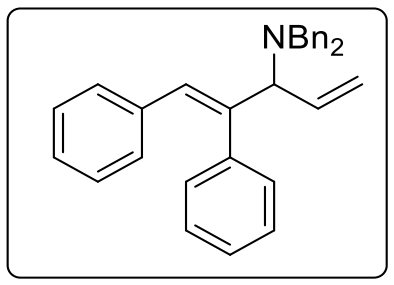

(4E)-N,N-Dibenzyl-1,2-diphenyl-penta-1,4-dien-3-amine (4an)

The title compound was prepared via general procedure B, purified by flash chromatography on silica gel eluting with in $\mathrm{CH}_{2} \mathrm{Cl}_{2} / \mathrm{PE}=1 / 3(\mathrm{Rf}=0.18)$, yellow oil, $48.2 \mathrm{mg}, 58 \%$ yield, ${ }^{1} \mathrm{H} \mathrm{NMR}\left(300 \mathrm{MHz}, \mathrm{CDCl}_{3}\right)$ $\delta$ 7.32-7.15 (m, 9H), 7.07-6.96 (m, 9H), 6.93-6.85 (m, 2H), $6.62(\mathrm{~d}, J=1.8 \mathrm{~Hz}, 1 \mathrm{H}), 6.24-5.95(\mathrm{~m}, 1 \mathrm{H})$, $5.50(\mathrm{dd}, J=10.2,2.1 \mathrm{~Hz}, 1 \mathrm{H}), 5.18(\mathrm{dd}, J=17.1,1.8 \mathrm{~Hz}, 1 \mathrm{H}), 4.11(\mathrm{dd}, J=9.3,1.8 \mathrm{~Hz}, 1 \mathrm{H}), 3.83(\mathrm{~d}, J$ $=13.8 \mathrm{~Hz}, 2 \mathrm{H}), 3.40(\mathrm{~d}, J=13.8 \mathrm{~Hz}, 2 \mathrm{H}) .{ }^{13} \mathrm{C} \mathrm{NMR}\left(75 \mathrm{MHz}, \mathrm{CDCl}_{3}\right) \delta 144.1,140.1,139.9,137.3,133.5$, $129.7,129.5,129.0,128.8,128.2,128.1,127.9,127.0,126.8,126.5,120.1,67.5,53.7$. HRMS (ESI) 
calculated for $\mathrm{C}_{31} \mathrm{H}_{30} \mathrm{~N}[\mathrm{M}+\mathrm{H}]^{+}:$416.2378, found: 416.2376 .

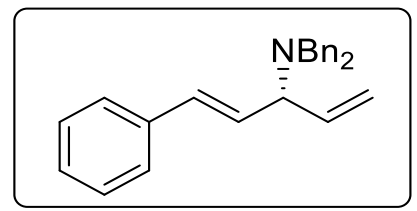

(3R,4E)- $N, N$-dibenzyl-1-phenylpenta-1,4-dien-3-amine (4aa')

4aa'was prepared via general procedure B with $(R)-\mathbf{L} 1$ was instead of ( $\mathrm{rac})-\mathbf{L} 1$, purified by flash chromatography on silica gel eluting with in $\mathrm{CH}_{2} \mathrm{Cl}_{2} / \mathrm{PE}=1 / 3(\mathrm{Rf}=0.22)$, colorless oil, $52.4 \mathrm{mg}, 78 \%$ yield, 99\% ee, ee value was determined by HPLC: Daicel Chiralpak OD-H column, n-hexane/i-PrOH = $99 / 1$, flow rate $1.0 \mathrm{~mL} / \mathrm{min}, \lambda=254 \mathrm{~nm}, \mathrm{t}_{\mathrm{R}}=4.22 \mathrm{~min}$ (major) and $3.80 \mathrm{~min}$ (minor). $[\alpha]_{\mathrm{D}}^{25}=+8.2^{\circ}(\mathrm{c}=$ $\left.0.1, \mathrm{CHCl}_{3}\right)$. The NMR analysis was same with $\mathbf{4 a h}$.

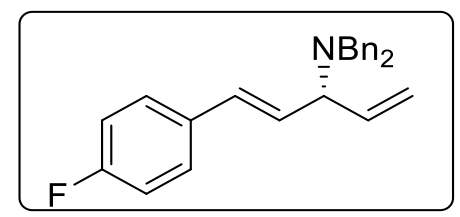

(4E)- $N, N$-dibenzyl-1-p-fluorophenyl penta-1,4-dien-3-amine (4ba')

4ba'was prepared via general procedure B with $(R)$-L1 was instead of ( $\mathrm{rac})$-L1, purified by flash chromatography on silica gel eluting with in $\mathrm{CH}_{2} \mathrm{Cl}_{2} / \mathrm{PE}=1 / 3(\mathrm{Rf}=0.25)$, colorless oil, $53.1 \mathrm{mg}, 74 \%$ yield, 95\% ee, ee value was determined by HPLC: Daicel Chiralpak OD-H column, n-hexane/i-PrOH = $99 / 1$, flow rate $1.0 \mathrm{~mL} / \mathrm{min}, \lambda=254 \mathrm{~nm}, \mathrm{t}_{\mathrm{R}}=4.42 \mathrm{~min}$ (major) and $3.88 \mathrm{~min}$ (minor). $[\alpha]_{\mathrm{D}}{ }^{25}=+97.5^{\circ}$ (c $\left.=0.1, \mathrm{CHCl}_{3}\right)$. The NMR analysis was same with 4ai.

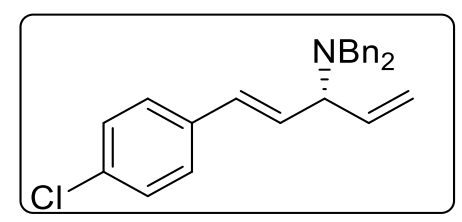

\section{(3R, 4E)- $N, N$-dibenzyl-1-p-chlorophenylpenta-1,4-dien-3-amine (4ca')}

4ca'was prepared via general procedure B with $(R)-\mathbf{L} 1$ was instead of ( $\mathrm{rac}$ )-L1, purified by flash chromatography on silica gel eluting with in $\mathrm{CH}_{2} \mathrm{Cl}_{2} / \mathrm{PE}=1 / 3(\mathrm{Rf}=0.25)$, colorless oil, $60.3 \mathrm{mg}, 80 \%$ yield, 96\% ee, ee value was determined by HPLC: Daicel Chiralpak OD-H column, $\mathrm{n}$-hexane/i-PrOH = $99 / 1$, flow rate $1.0 \mathrm{~mL} / \mathrm{min}, \lambda=254 \mathrm{~nm}, \mathrm{t}_{\mathrm{R}}=4.97 \mathrm{~min}$ (major) and $4.13 \mathrm{~min}$ (minor). $[\alpha]_{\mathrm{D}}{ }^{25}=+154.1^{\circ}(\mathrm{c}$ $\left.=0.2, \mathrm{CHCl}_{3}\right) .{ }^{1} \mathrm{H} \mathrm{NMR}\left(400 \mathrm{MHz}, \mathrm{CDCl}_{3}\right) \delta 7.41(\mathrm{~d}, J=7.2 \mathrm{~Hz}, 4 \mathrm{H}), 7.35-7.20(\mathrm{~m}, 10 \mathrm{H}), 6.40(\mathrm{~d}, J=$ $16.4 \mathrm{~Hz}, 1 \mathrm{H}), 6.30(\mathrm{dd}, J=16.4,6.2 \mathrm{~Hz}, 1 \mathrm{H}), 6.02(\mathrm{ddd}, J=17.2,10.4,6.4 \mathrm{~Hz}, 1 \mathrm{H}), 5.33$ (dt, $J=10.4$, $1.2 \mathrm{~Hz}, 1 \mathrm{H}), 5.17(\mathrm{dt}, J=17.2,1.2 \mathrm{~Hz}, 1 \mathrm{H}), 3.85(\mathrm{t}, J=6.0 \mathrm{~Hz}, 1 \mathrm{H}), 3.72-3.61(\mathrm{~m}, 4 \mathrm{H}) .{ }^{13} \mathrm{C} \mathrm{NMR}(75$ $\left.\mathrm{MHz}, \mathrm{CDCl}_{3}\right) \delta 140.3,137.3,135.7,133.2,131.7,130.2,128.8,128.7,128.4,127.7,126.9,118.4,63.3$, 54.1. HRMS (ESI) calculated for $\mathrm{C}_{25} \mathrm{H}_{25} \mathrm{ClN}[\mathrm{M}+\mathrm{H}]^{+}: 374.1676$, found: 374.1674 . 


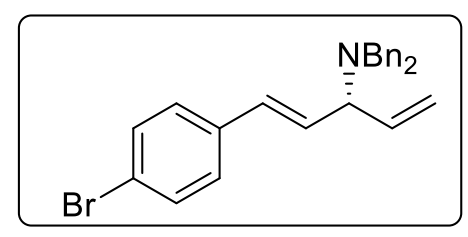

(4E)- $N, N$-dibenzyl-1-p-bromophenylpenta-1,4-dien-3-amine (4da')

4da' was prepared via general procedure B with $(R)-\mathbf{L} 1$ was instead of ( $\mathrm{rac})$-L1, purified by flash chromatography on silica gel eluting with in $\mathrm{CH}_{2} \mathrm{Cl}_{2} / \mathrm{PE}=1 / 3(\mathrm{Rf}=0.24)$, colorless oil, $65.8 \mathrm{mg}, 78 \%$ yield, 97\% ee, ee value was determined by HPLC: Daicel Chiralpak OD-H column, n-hexane/i-PrOH = $99 / 1$, flow rate $1.0 \mathrm{~mL} / \mathrm{min}, \lambda=254 \mathrm{~nm}, \mathrm{t}_{\mathrm{R}}=4.85 \mathrm{~min}$ (major) and $3.98 \mathrm{~min}$ (minor). $[\alpha]_{\mathrm{D}}{ }^{25}=+97.5^{\circ}$ (c $\left.=0.1, \mathrm{CHCl}_{3}\right)$. The NMR analysis was same with $\mathbf{4 a j}$.

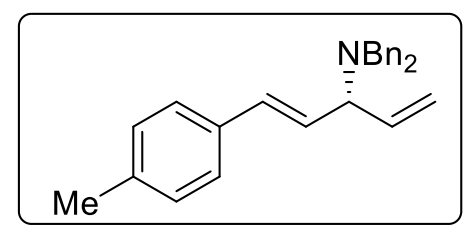

(4E)- $N, N$-dibenzyl-1-p-bromophenylpenta-1,4-dien-3-amine (4ea')

4ea'was prepared via general procedure B with $(R)-\mathbf{L 1}$ was instead of ( $\mathrm{rac})$-L1, purified by flash chromatography on silica gel eluting with in $\mathrm{CH}_{2} \mathrm{Cl}_{2} / \mathrm{PE}=1 / 3(\mathrm{Rf}=0.25)$, colorless oil, $49.8 \mathrm{mg}, 70 \%$ yield, $87 \%$ ee, ee value was determined by HPLC: Daicel Chiralpak OD-H column, n-hexane/i-PrOH = $99 / 1$, flow rate $1.0 \mathrm{~mL} / \mathrm{min}, \lambda=254 \mathrm{~nm}, \mathrm{t}_{\mathrm{R}}=4.01 \mathrm{~min}$ (major) and $3.64 \mathrm{~min}$ (minor). $[\alpha]_{\mathrm{D}}{ }^{25}=+97.5^{\circ}$ (c $\left.=0.1, \mathrm{CHCl}_{3}\right)$. The NMR analysis was same with $\mathbf{4 a k}$

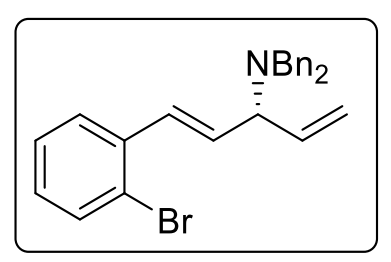

(3R,4E)- $N, N$-dibenzyl-1-o-bromophenyl-penta-2,4-dien-3-amine (4fa')

4fa'was prepared via general procedure B with $(R)$-L1 was instead of ( $\mathrm{rac}$ )-L1, purified by flash chromatography on silica gel eluting with in $\mathrm{CH}_{2} \mathrm{Cl}_{2} / \mathrm{PE}=1 / 3(\mathrm{Rf}=0.20)$, colorless oil, $68.6 \mathrm{mg}, 82 \%$ yield, 92\% ee, ee value was determined by HPLC: Daicel Chiralpak OD-H column, n-hexane/i-PrOH = $99 / 1$, flow rate $1.0 \mathrm{~mL} / \mathrm{min}, \lambda=254 \mathrm{~nm}, \mathrm{t}_{\mathrm{R}}=4.96 \mathrm{~min}$ (major) and $4.13 \mathrm{~min}$ (minor). $[\alpha]_{\mathrm{D}}^{25}=+7.5^{\circ}(\mathrm{c}=$ $0.1, \mathrm{CHCl}_{3}$ ). The NMR analysis was same with $\mathbf{4 a l}$.

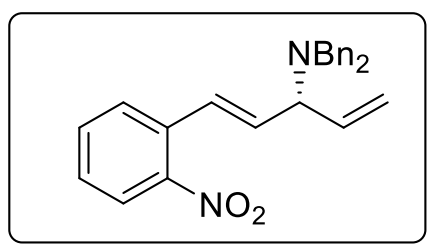

(4E)-N, $N$-dibenzyl-1-o-nitrylphenyl-penta-2,4-dien-3-amine (4ga')

4ga' was prepared via general procedure B with $(R)-\mathbf{L} 1$ was instead of ( $r a c)-\mathbf{L 1}$, purified by flash 
chromatography on silica gel eluting with in EtOAc/PE $=1 / 10(\mathrm{Rf}=0.32)$, colorless oil, $44.8 \mathrm{mg}, 58 \%$ yield, 96\% ee, ee value was determined by HPLC: Daicel Chiralpak OD-H column, n-hexane/i-PrOH = $99 / 1$, flow rate $1.0 \mathrm{~mL} / \mathrm{min}, \lambda=254 \mathrm{~nm}, \mathrm{t}_{\mathrm{R}}=8.96 \mathrm{~min}$ (major) and $5.87 \mathrm{~min}$ (minor). $[\alpha]_{\mathrm{D}}{ }^{25}=+18.2^{\circ}$ (c $\left.=0.1, \mathrm{CHCl}_{3}\right) .{ }^{1} \mathrm{H} \mathrm{NMR}\left(400 \mathrm{MHz}, \mathrm{CDCl}_{3}\right) \delta 7.93(\mathrm{~d}, J=8.0 \mathrm{~Hz}, 1 \mathrm{H}), 7.55(\mathrm{~d}, J=4.0 \mathrm{~Hz}, 2 \mathrm{H}), 7.43(\mathrm{~d}$, $J=7.2 \mathrm{~Hz}, 4 \mathrm{H}), 7.40-7.35(\mathrm{~m}, 1 \mathrm{H}), 7.32$ (t, $J=7.6 \mathrm{~Hz}, 4 \mathrm{H}), 7.26-7.20$ (m, 2H), 6.94 (dd, $J=16.0,1.2$ $\mathrm{Hz}, 1 \mathrm{H}), 6.29$ (dd, $J=16.0,6.4 \mathrm{~Hz}, 1 \mathrm{H}), 6.04(\mathrm{ddd}, J=17.2,10.4,6.4 \mathrm{~Hz}, 1 \mathrm{H}), 5.36(\mathrm{~d}, J=10.4 \mathrm{~Hz}, 1 \mathrm{H})$, $5.21(\mathrm{dt}, J=17.2,1.2 \mathrm{~Hz}, 1 \mathrm{H}), 3.91(\mathrm{t}, J=6.4 \mathrm{~Hz}, 1 \mathrm{H}), 3.71(\mathrm{~d}, J=14.0 \mathrm{~Hz}, 2 \mathrm{H}), 3.67$ (d, $J=14.0 \mathrm{~Hz}$, 2H). ${ }^{13} \mathrm{C}$ NMR $\left(100 \mathrm{MHz}, \mathrm{CDCl}_{3}\right) \delta 147.81,140.14,136.91,135.14,133.18,129.04,128.76,128.56$, $128.42,128.12,127.00,124.65,118.81,63.21,54.17$. HRMS (ESI) calculated for $\mathrm{C}_{25} \mathrm{H}_{25} \mathrm{~N}_{2} \mathrm{O}_{2}[\mathrm{M}+\mathrm{H}]^{+}$: 385.1916, found: 385.1911 .

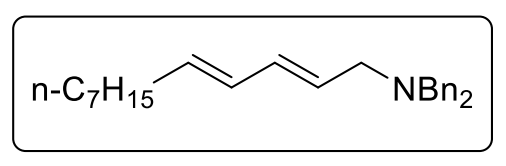

\section{(2E,4E)- $N, N$-Dibenzyl-dodeca-2,4-dien-1-amine (5aa)}

The title compound was prepared via general procedure $\mathrm{C}$, purified by flash chromatography on silica gel eluting with in $\mathrm{EtOAc} / \mathrm{PE}=1 / 10(\mathrm{Rf}=0.32)$, yellow oil, $36.5 \mathrm{mg}, 51 \%$ yield, E,E/E,Z = 16.7/1, E,Eisomer: ${ }^{1} \mathrm{H} \mathrm{NMR}\left(400 \mathrm{MHz}, \mathrm{CDCl}_{3}\right) \delta 7.36(\mathrm{~d}, J=7.2 \mathrm{~Hz}, 4 \mathrm{H}), 7.30(\mathrm{t}, J=7.2 \mathrm{~Hz}, 4 \mathrm{H}), 7.22(\mathrm{t}, J=7.2$ $\mathrm{Hz}, 2 \mathrm{H}), 6.14(\mathrm{dd}, J=15.2,10.4 \mathrm{~Hz}, 1 \mathrm{H}), 6.03$ (dd, $J=15.2,10.4 \mathrm{~Hz}, 1 \mathrm{H}), 5.73-5.48(\mathrm{~m}, 2 \mathrm{H}), 3.60-3.48$ $(\mathrm{m}, 4 \mathrm{H}), 3.06(\mathrm{~d}, J=6.6 \mathrm{~Hz}, 2 \mathrm{H}), 2.06(\mathrm{q}, J=7.0 \mathrm{~Hz}, 2 \mathrm{H}), 1.41-1.18(\mathrm{~m}, 10 \mathrm{H}), 0.87$ (t, $J=6.8 \mathrm{~Hz}, 3 \mathrm{H})$. ${ }^{13} \mathrm{C} \mathrm{NMR}\left(100 \mathrm{MHz}, \mathrm{CDCl}_{3}\right) \delta 139.9,134.3,133.4,129.9,128.9,128.6,128.3,126.9,57.9,55.5,32.8$, 31.9, 29.4, 29.34, 29.32, 22.8, 14.2. HRMS (ESI) calculated for $\mathrm{C}_{26} \mathrm{H}_{36} \mathrm{~N}[\mathrm{M}+\mathrm{H}]^{+}: 362.2848$, found: 362.2835 .

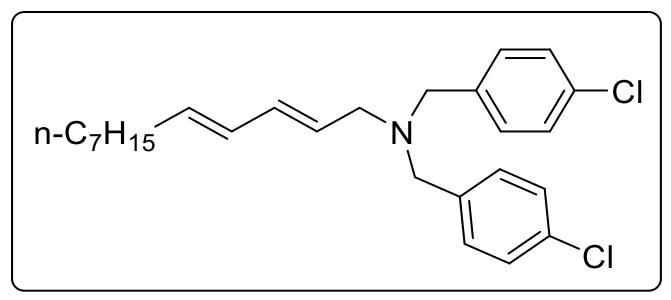

\section{$(2 E, 4 E)-N, N$-Di-(p-chlorobenzyl)-dodeca-2,4-dien-1-amine (5ab)}

The title compound was prepared via general procedure $\mathrm{C}$, purified by flash chromatography on silica gel eluting with in EtOAc/PE = 1/10 (Rf=0.32), yellow oil, $38.6 \mathrm{mg}, 45 \%$ yield, E,E/E,Z > 20/1, ${ }^{1} \mathrm{H}$ NMR $\left(400 \mathrm{MHz}, \mathrm{CDCl}_{3}\right) \delta 7.29(\mathrm{br}, 8 \mathrm{H}), 6.24-5.86(\mathrm{~m}, 2 \mathrm{H}), 5.64(\mathrm{ddd}, J=25.8,14.0,6.8 \mathrm{~Hz}, 2 \mathrm{H}), 3.51$ (s, $4 \mathrm{H}), 3.04(\mathrm{~d}, J=6.6 \mathrm{~Hz}, 2 \mathrm{H}), 2.08(\mathrm{q}, J=7.2 \mathrm{~Hz}, 2 \mathrm{H}), 1.31-1.28(\mathrm{~m}, 10 \mathrm{H}), 0.89(\mathrm{t}, J=6.5 \mathrm{~Hz}, 3 \mathrm{H}) .{ }^{13} \mathrm{C}$ NMR $\left(100 \mathrm{MHz}, \mathrm{CDCl}_{3}\right) \delta 138.1,134.7,133.7,132.5,130.0,129.6,128.4,127.8,57.0,55.3,32.6,31.8$, 29.29, 29.21, 29.19, 22.7, 14.1. HRMS (ESI) calculated for $\mathrm{C}_{26} \mathrm{H}_{34} \mathrm{Cl}_{2} \mathrm{~N}[\mathrm{M}+\mathrm{H}]^{+}:$430.2068, found: 430.2065 . 


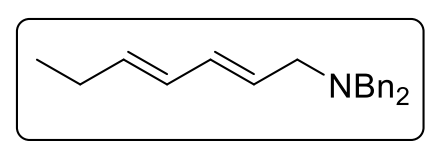

$(2 E, 4 E)-N, N$-Dibenzyl-hepta-2,4-dien-1-amine (5ac)

The title compound was prepared via general procedure $\mathrm{C}$, purified by flash chromatography on silica gel eluting with in EtOAc/PE = 1/10 ( $\mathrm{Rf}=0.38)$, colorless oil, $35.9 \mathrm{mg}, 61 \%$ yield, E,E/E,Z = 6/1, E,Eisomer: ${ }^{1} \mathrm{H}$ NMR (400 MHz, $\left.\mathrm{CDCl}_{3}\right) \delta$ 7.40-7.13 (m, 10H), 6.17-6.00 (m, 2H), 5.70-5.61 (m, 2H), 3.56 (s, 4H), 3.07 (d, $J=6.6 \mathrm{~Hz}, 2 \mathrm{H}), 2.09$ (p, $J=7.2 \mathrm{~Hz}, 1 \mathrm{H}), 1.00$ (t, $J=7.5 \mathrm{~Hz}, 3 \mathrm{H}) .{ }^{13} \mathrm{C} \mathrm{NMR}(100 \mathrm{MHz}$, $\left.\mathrm{CDCl}_{3}\right) \delta 139.9,135.7,133.4,129.0,128.9,128.7,128.3,126.9,57.9,55.4,25.7,13.6$. HRMS (ESI) calculated for $\mathrm{C}_{21} \mathrm{H}_{26} \mathrm{~N}[\mathrm{M}+\mathrm{H}]^{+}:$292.2065, found: 292.2062.

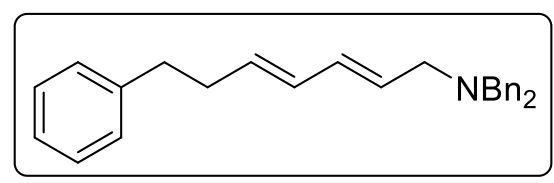

\section{$(2 E, 4 E)-N, N$-Dibenzyl-7-phenyl-hepta-2,4-dien-1-amine (5ad)}

The title compound was prepared via general procedure $\mathrm{C}$, purified by flash chromatography on silica gel eluting with in EtOAc/PE = 1/10 ( Rf = 0.36), colorless oil, $41.0 \mathrm{mg}, 55 \%$ yield, E,E/E,Z = 5/1, E,Eisomer: ${ }^{1} \mathrm{H}$ NMR (300 MHz, $\left.\mathrm{CDCl}_{3}\right) \delta$ 7.37-7.17 (m, 15H), 6.25-6.00 (m, 2H), $5.77-5.56(\mathrm{~m}, 2 \mathrm{H}), 3.56$ $(\mathrm{s}, 4 \mathrm{H}), 3.06(\mathrm{~d}, J=6.6 \mathrm{~Hz}, 2 \mathrm{H}), 2.70(\mathrm{dd}, J=9.0,6.6 \mathrm{~Hz}, 2 \mathrm{H}), 2.39(\mathrm{dd}, J=15.0,7.5 \mathrm{~Hz}, 2 \mathrm{H}) .{ }^{13} \mathrm{C} \mathrm{NMR}$ $\left(75 \mathrm{MHz}, \mathrm{CDCl}_{3}\right) \delta 141.8,139.7,133.1,132.8,130.4,129.2,128.8,128.4,128.3,128.2,126.8,125.9$, 57.8, 55.3, 35.8, 34.5. HRMS (ESI) calculated for $\mathrm{C}_{27} \mathrm{H}_{30} \mathrm{~N}[\mathrm{M}+\mathrm{H}]^{+}: 368.2378$, found: 368.2374.

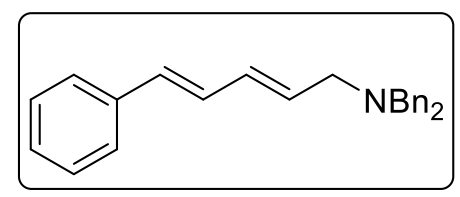

(4E)- $N, N$-dibenzyl-5-phenyl-penta-2,4-dien-1-amine (5ae) ${ }^{[3]}$

The title compound was prepared via general procedure $\mathrm{C}$, purified by flash chromatography on silica gel eluting with in EtOAc/PE = 1/10 ( Rf = 0.32), yellow oil, $40.8 \mathrm{mg}, 60 \%$ yield, E,E/E,Z = 11.8/1, E,Eisomer: ${ }^{1} \mathrm{H}$ NMR $\left(400 \mathrm{MHz}, \mathrm{CDCl}_{3}\right) \delta$ 7.44-7.42 (m, 6H), $7.35(\mathrm{~d}, J=7.5 \mathrm{~Hz}, 6 \mathrm{H}), 7.31-7.22(\mathrm{~m}, 3 \mathrm{H})$, $6.83(\mathrm{dd}, J=15.6,10.4 \mathrm{~Hz}, 1 \mathrm{H}), 6.54(\mathrm{~d}, J=15.6 \mathrm{~Hz}, 1 \mathrm{H}), 6.40(\mathrm{dd}, J=15.2,10.4 \mathrm{~Hz}, 1 \mathrm{H}), 6.06-5.88$ $(\mathrm{m}, 1 \mathrm{H}), 3.65(\mathrm{~s}, 4 \mathrm{H}), 3.20(\mathrm{~d}, J=6.4 \mathrm{~Hz}, 2 \mathrm{H})$. The ${ }^{1} \mathrm{H}$ NMR was same with the reported literature.

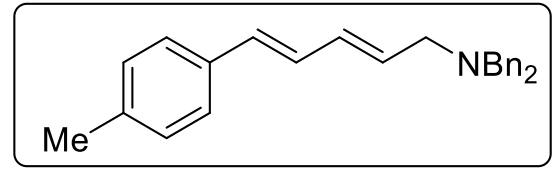

\section{(2E,4E)- $N, N$-Dibenzyl-5-p-methylphenyl-penta-2,4-dien-1-amine (5af)}

The title compound was prepared via general procedure $\mathrm{C}$, purified by flash chromatography on silica gel eluting with in EtOAc/PE = 1/10 ( $\mathrm{Rf}=0.33)$, colorless oil, $43.8 \mathrm{mg}, 62 \%$ yield, E,E/E,Z = 10/1, E,Eisomer: ${ }^{1} \mathrm{H} \mathrm{NMR}\left(300 \mathrm{MHz}, \mathrm{CDCl}_{3}\right) \delta 7.46-7.17(\mathrm{~m}, 12 \mathrm{H}), 7.10(\mathrm{~d}, J=8.1 \mathrm{~Hz}, 2 \mathrm{H}), 6.74(\mathrm{dd}, J=15.5$, 
$10.2 \mathrm{~Hz}, 1 \mathrm{H}), 6.46(\mathrm{~d}, J=15.5 \mathrm{~Hz}, 1 \mathrm{H}), 6.33(\mathrm{dd}, J=15.5,10.2 \mathrm{~Hz}, 1 \mathrm{H}), 5.99-5.77(\mathrm{~m}, 1 \mathrm{H}), 3.59(\mathrm{~s}, 4 \mathrm{H})$, $3.14(\mathrm{~d}, J=6.6 \mathrm{~Hz}, 2 \mathrm{H}), 2.32(\mathrm{~s}, 3 \mathrm{H}) .{ }^{13} \mathrm{C} \mathrm{NMR}\left(75 \mathrm{MHz}, \mathrm{CDCl}_{3}\right) \delta$ 139.7, 137.3, 134.6, 133.3, 131.5, 131.4, 129.3, 128.8, 128.3, 127.8, 126.9, 126.2, 57.9, 55.4, 21.3. HRMS (ESI) calculated for $\mathrm{C}_{26} \mathrm{H}_{28} \mathrm{~N}$ $[\mathrm{M}+\mathrm{H}]^{+}:$354.2222, found: 354.2224 .

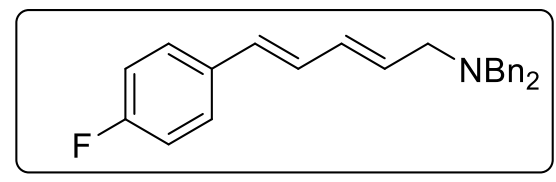

\section{(2E,4E)- $N, N$-Dibenzyl-5-p-fluorophenyl-penta-2,4-dien-1-amine (5ag)}

The title compound was prepared via general procedure $\mathrm{C}$, purified by flash chromatography on silica gel eluting with in EtOAc/PE = 1/10 (Rf=0.32), colorless oil, $48.1 \mathrm{mg}, 68 \%$ yield, E,E/E,Z = 13.3/1, E,Eisomer: ${ }^{1} \mathrm{H}$ NMR $\left(300 \mathrm{MHz}, \mathrm{CDCl}_{3}\right) \delta$ 7.44-7.32 (m, 10H), 7.25-7.14 (m, 2H), 7.06-6.92 (m, 2H), 6.69 $(\mathrm{dd}, J=15.6,10.4 \mathrm{~Hz}, 1 \mathrm{H}), 6.44(\mathrm{~d}, J=15.6 \mathrm{~Hz}, 1 \mathrm{H}), 6.38-6.27(\mathrm{~m}, 1 \mathrm{H}), 5.90$ (d, $J=15.3 \mathrm{~Hz}, 1 \mathrm{H}), 3.66-$ $3.55(\mathrm{~m}, 4 \mathrm{H}), 3.15(\mathrm{~d}, J=6.6 \mathrm{~Hz}, 2 \mathrm{H}) .{ }^{13} \mathrm{C} \mathrm{NMR}\left(75 \mathrm{MHz}, \mathrm{CDCl}_{3}\right) \delta 139.7,133.7,132.9,132.3,130.3$, 128.9, $128.6(\mathrm{~d}, J=2.4 \mathrm{~Hz}), 128.4,127.9,127.8,127.0,115.7$ (d, $J=21.7 \mathrm{~Hz}), 58.0,55.5$. HRMS (ESI) calculated for $\mathrm{C}_{25} \mathrm{H}_{25} \mathrm{FN}[\mathrm{M}+\mathrm{H}]^{+}: 358.1971$, found: 358.1975.

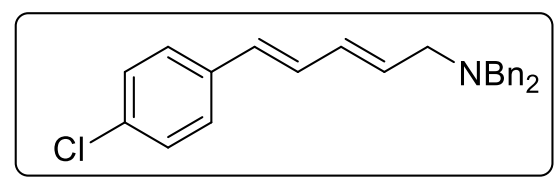

\section{(2E,4E)- $N, N$-Dibenzyl-5-p-chlorophenyl-penta-2,4-dien-1-amine (5ah)}

The title compound was prepared via general procedure $\mathrm{C}$, purified by flash chromatography on silica gel eluting with in EtOAc/PE = 1/10 (Rf=0.33), colorless oil, $51.8 \mathrm{mg}, 70 \%$ yield, E,E/E,Z = 11.8/1, E,Eisomer: ${ }^{1} \mathrm{H}$ NMR $\left(300 \mathrm{MHz}, \mathrm{CDCl}_{3}\right) \delta$ 7.59-7.06 (m, 14H), $6.74(\mathrm{dd}, J=15.6,10.5 \mathrm{~Hz}, 1 \mathrm{H}), 6.42(\mathrm{~d}, J=$ $15.6 \mathrm{~Hz}, 1 \mathrm{H}), 6.33(\mathrm{dd}, J=15.3,10.5 \mathrm{~Hz}, 1 \mathrm{H}), 5.92(\mathrm{dt}, J=15.3,6.6 \mathrm{~Hz}, 1 \mathrm{H}), 3.59$ (s, 4H), 3.15 (d, $J=$ $6.5 \mathrm{~Hz}, 2 \mathrm{H}) .{ }^{13} \mathrm{C} \mathrm{NMR}\left(75 \mathrm{MHz}, \mathrm{CDCl}_{3}\right) \delta 139.7,136.0,133.0,132.8,130.2,129.4,129.0,128.9(2 \mathrm{C})$, 128.4, 127.5, 127.0, 58.0, 55.5. HRMS (ESI) calculated for $\mathrm{C}_{25} \mathrm{H}_{25} \mathrm{CIN}[\mathrm{M}+\mathrm{H}]^{+}:$374.1676, found: 374.1674 .

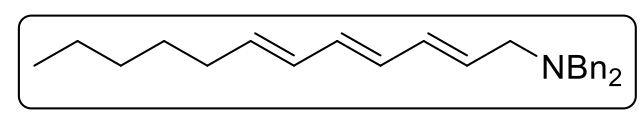

\section{$(2 E, 4 E, 6 E)-N, N$-Dibenzyl-dodeca-2,4,6-trien-1-amine (5ai)}

The title compound was prepared via general procedure $\mathrm{C}$, purified by flash chromatography on silica gel eluting with in EtOAc/PE = 1/10 (Rf=0.32), yellow oil , $19.8 \mathrm{mg}, 38 \%$ yield, $(\mathrm{E}, \mathrm{E}, \mathrm{E}) /(\mathrm{E}, \mathrm{E}, \mathrm{Z})=6.1 / 1$, E,E,E-isomer: ${ }^{1} \mathrm{H}$ NMR (400 MHz, $\left.\mathrm{CDCl}_{3}\right) \delta 7.36(\mathrm{~d}, J=7.2 \mathrm{~Hz}, 4 \mathrm{H}), 7.30(\mathrm{t}, J=7.2 \mathrm{~Hz}, 4 \mathrm{H}), 7.22(\mathrm{t}, J$ $=7.2 \mathrm{~Hz}, 2 \mathrm{H}), 6.23-6.01(\mathrm{~m}, 4 \mathrm{H}), 5.7845 .64(\mathrm{~m}, 2 \mathrm{H}), 3.81-3.43(\mathrm{~m}, 4 \mathrm{H}), 3.09(\mathrm{~d}, J=6.8 \mathrm{~Hz}, 1 \mathrm{H}), 2.08$ $(\mathrm{dd}, J=14.0,7.2 \mathrm{~Hz}, 2 \mathrm{H}), 1.49-1.14(\mathrm{~m}, 6 \mathrm{H}), 0.88(\mathrm{t}, J=6.7 \mathrm{~Hz}, 3 \mathrm{H}) .{ }^{13} \mathrm{C} \mathrm{NMR}\left(100 \mathrm{MHz}, \mathrm{CDCl}_{3}\right) \delta$ $139.8,135.7,133.3,132.5,130.6$, 130.4, 130.2, 128.9, 128.3, 126.9, 57.9, 55.5, 32.9, 31.5, 29.1, 22.7, 
14.2. HRMS (ESI) calculated for $\mathrm{C}_{26} \mathrm{H}_{34} \mathrm{~N}[\mathrm{M}+\mathrm{H}]^{+}: 260.2691$, found: 260.2695 .

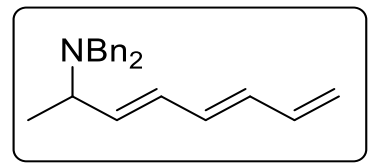

$(3 E, 5 E)-N, N$-Dibenzyl-octa-1,3,5-trien-7-amine (6aa)

The title compound was prepared via general procedure A, purified by flash chromatography on silica gel eluting with in DCM/PE $=1 / 2(\mathrm{Rf}=0.30)$, yellow oil, $51.5 \mathrm{mg}, 84 \%$ yield, ${ }^{1} \mathrm{H}$ NMR $\left(300 \mathrm{MHz}, \mathrm{CDCl}_{3}\right)$ $\delta 7.38(\mathrm{~d}, J=7.1 \mathrm{~Hz}, 4 \mathrm{H}), 7.33-7.12(\mathrm{~m}, 6 \mathrm{H}), 6.49-6.30(\mathrm{~m}, 1 \mathrm{H}), 6.29-6.02(\mathrm{~m}, 3 \mathrm{H}), 5.83(\mathrm{dd}, J=15.0$, $6.6 \mathrm{~Hz}, 1 \mathrm{H}), 5.21(\mathrm{dd}, J=16.8,1.5 \mathrm{~Hz}, 1 \mathrm{H}), 5.08(\mathrm{dd}, J=9.9,1.5 \mathrm{~Hz}, 1 \mathrm{H}), 3.64(\mathrm{~d}, J=14.1 \mathrm{~Hz}, 2 \mathrm{H})$, $3.52(\mathrm{~d}, J=14.1 \mathrm{~Hz}, 2 \mathrm{H}), 3.41-3.32(\mathrm{~m}, 1 \mathrm{H}), 1.20(\mathrm{~d}, J=6.9 \mathrm{~Hz}, 3 \mathrm{H}) .{ }^{13} \mathrm{C} \mathrm{NMR}\left(75 \mathrm{MHz}, \mathrm{CDCl}_{3}\right) \delta$ $140.7,137.1,136.6,133.2,132.5,131.2,128.6$, 128.3, 126.8, 117.1, 54.6, 53.7, 15.6. HRMS (ESI) calculated for $\mathrm{C}_{22} \mathrm{H}_{26} \mathrm{~N}[\mathrm{M}+\mathrm{H}]^{+}:$304.2065, found: 304.2066 .

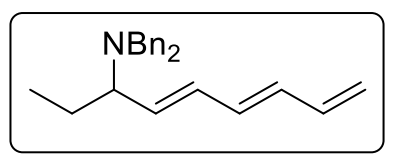

$(3 E, 5 E)-N, N$-Dibenzyl-nuna-1,3,5-trien-7-amine (6ab)

The title compound was prepared via general procedure A, purified by flash chromatography on silica gel eluting with in $\mathrm{CH}_{2} \mathrm{Cl}_{2} / \mathrm{PE}=1 / 2(\mathrm{Rf}=0.30)$, yellow oil, $56.5 \mathrm{mg}, 89 \%$ yield. ${ }^{1} \mathrm{H} \mathrm{NMR}\left(400 \mathrm{MHz}, \mathrm{CDCl}_{3}\right)$ $\delta 7.38(\mathrm{~d}, J=7.2 \mathrm{~Hz}, 4 \mathrm{H}), 7.30(\mathrm{t}, J=7.2 \mathrm{~Hz}, 4 \mathrm{H}), 7.21(\mathrm{t}, J=7.2 \mathrm{~Hz}, 2 \mathrm{H}), 6.40(\mathrm{dt}, J=16.8,10.0 \mathrm{~Hz}$, $1 \mathrm{H}), 6.29(\mathrm{dd}, J=14.8,9.6 \mathrm{~Hz}, 1 \mathrm{H}), 6.21(\mathrm{dd}, J=14.8,9.6 \mathrm{~Hz}, 1 \mathrm{H}), 6.06(\mathrm{dd}, J=15.2,9.6 \mathrm{~Hz}, 1 \mathrm{H}), 5.72$ $(\mathrm{dd}, J=15.2,8.8 \mathrm{~Hz}, 1 \mathrm{H}), 5.23(\mathrm{~d}, J=16.8 \mathrm{~Hz}, 1 \mathrm{H}), 5.11(\mathrm{~d}, J=10.8 \mathrm{~Hz}, 1 \mathrm{H}), 3.80(\mathrm{~d}, J=14.0 \mathrm{~Hz}, 2 \mathrm{H})$, $3.36(\mathrm{~d}, J=14.0 \mathrm{~Hz}, 2 \mathrm{H}), 2.96(\mathrm{q}, J=7.6 \mathrm{~Hz}, 1 \mathrm{H}), 1.83-1.64(\mathrm{~m}, 1 \mathrm{H}), 1.52-1.40(\mathrm{~m}, 1 \mathrm{H}), 0.89(\mathrm{t}, J=7.6$ $\mathrm{Hz}, 3 \mathrm{H}) .{ }^{13} \mathrm{C} \mathrm{NMR}\left(100 \mathrm{MHz}, \mathrm{CDCl}_{3}\right) \delta 140.7,137.1,133.5,133.2,133.1,132.6,128.7,128.3,126.8$, 117.3, 61.9, 53.8, 25.5, 11.5. HRMS (ESI) calculated for $\mathrm{C}_{23} \mathrm{H}_{28} \mathrm{~N}[\mathrm{M}+\mathrm{H}]^{+}:$318.2222, found: 318.2220.

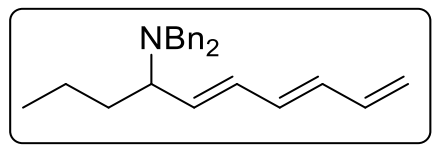

$(3 E, 5 E)-N, N$-dibenzyl-deca-1,3,5-trien-7-amine (6ac)

The title compound was prepared via general procedure A, purified by flash chromatography on silica gel eluting with in $\mathrm{CH}_{2} \mathrm{Cl}_{2} / \mathrm{PE}=1 / 3(\mathrm{Rf}=0.20)$, yellow oil, $62.1 \mathrm{mg}, 94 \%$ yield, ${ }^{1} \mathrm{H} \mathrm{NMR}\left(300 \mathrm{MHz}, \mathrm{CDCl}_{3}\right)$ $\delta$ 7.39-7.18 (m, 10H), $6.40(\mathrm{dt}, J=16.8,9.9 \mathrm{~Hz}, 1 \mathrm{H}), 6.33-6.16(\mathrm{~m}, 2 \mathrm{H}), 6.05(\mathrm{dd}, J=15.3,9.3 \mathrm{~Hz}, 1 \mathrm{H})$, $5.73(\mathrm{dd}, J=15.3,9.0 \mathrm{~Hz}, 1 \mathrm{H}), 5.23(\mathrm{dd}, J=16.8,1.5 \mathrm{~Hz}, 1 \mathrm{H}), 5.10(\mathrm{dd}, J=9.9,1.5 \mathrm{~Hz}, 1 \mathrm{H}), 3.79(\mathrm{~d}, J$ $=13.8 \mathrm{~Hz}, 2 \mathrm{H}), 3.35(\mathrm{~d}, J=13.8 \mathrm{~Hz}, 2 \mathrm{H}), 3.07$ (q, $7.2 \mathrm{~Hz}, 1 \mathrm{H}), 1.77-1.59(\mathrm{~m}, 1 \mathrm{H}), 1.52-1.17(\mathrm{~m}, 3 \mathrm{H})$, $0.80(\mathrm{t}, J=7.2 \mathrm{~Hz}, 3 \mathrm{H}) .{ }^{13} \mathrm{C} \mathrm{NMR}\left(75 \mathrm{MHz}, \mathrm{CDCl}_{3}\right) \delta 140.7,137.1,133.7,133.1,133.0,132.6,128.8$, $128.3,126.8,117.3,59.9,53.8,34.9,19.9,14.2$. HRMS (ESI) calculated for $\mathrm{C}_{24} \mathrm{H}_{30} \mathrm{~N}[\mathrm{M}+\mathrm{H}]^{+}: 332.2378$, 
found: 332.2382 .

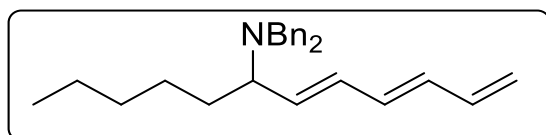

\section{$(3 E, 5 E)-N, N$-Dibenzyl-dodeca-1,3,5-trien-7-amine (6ad)}

The title compound was prepared via general procedure A, purified by flash chromatography on silica gel eluting with in $\mathrm{CH}_{2} \mathrm{Cl}_{2} / \mathrm{PE}=1 / 2(\mathrm{Rf}=0.32)$, yellow oil, $57.5 \mathrm{mg}, 89 \%$ yield, ${ }^{1} \mathrm{H} \mathrm{NMR}\left(400 \mathrm{MHz}, \mathrm{CDCl}_{3}\right)$ $\delta 7.37(\mathrm{~d}, J=7.2 \mathrm{~Hz}, 4 \mathrm{H}), 7.30(\mathrm{t}, J=7.2 \mathrm{~Hz}, 4 \mathrm{H}), 7.21(\mathrm{t}, J=7.2 \mathrm{~Hz}, 2 \mathrm{H}), 6.40(\mathrm{dt}, J=16.8,10.0 \mathrm{~Hz}$, $1 \mathrm{H}), 6.29(\mathrm{dd}, J=14.8,10.0 \mathrm{~Hz}, 1 \mathrm{H}), 6.21(\mathrm{dd}, J=14.8,10.0 \mathrm{~Hz}, 1 \mathrm{H}), 6.06(\mathrm{dd}, J=15.2,9.6 \mathrm{~Hz}, 1 \mathrm{H})$, $5.73(\mathrm{dd}, J=15.2,8.8 \mathrm{~Hz}, 1 \mathrm{H}), 5.23(\mathrm{~d}, J=16.8 \mathrm{~Hz}, 1 \mathrm{H}), 5.10(\mathrm{~d}, J=10.0 \mathrm{~Hz}, 1 \mathrm{H}), 3.79$ (d, $J=14.0 \mathrm{~Hz}$, 2H), $3.35(\mathrm{~d}, J=14.0 \mathrm{~Hz}, 2 \mathrm{H}), 3.05$ (q, $J=8.0 \mathrm{~Hz}, 1 \mathrm{H}), 1.74-1.65(\mathrm{~m}, 1 \mathrm{H}), 1.46-1.31(\mathrm{~m}, 2 \mathrm{H}), 1.30-1.21$ $(\mathrm{m}, 3 \mathrm{H}), 1.20-1.04(\mathrm{~m}, 2 \mathrm{H}), 0.84(\mathrm{t}, J=7.2 \mathrm{~Hz}, 3 \mathrm{H}) .{ }^{13} \mathrm{C} \mathrm{NMR}\left(75 \mathrm{MHz}, \mathrm{CDCl}_{3}\right) \delta 140.7,137.1,133.8$, 133.1, 133.0, 132.6, 128.8, 128.3, 126.8, 117.2, 60.0, 53.8, 32.5, 31.9, 26.3, 22.8, 14.2. HRMS (ESI) calculated for $\mathrm{C}_{26} \mathrm{H}_{34} \mathrm{~N}[\mathrm{M}+\mathrm{H}]^{+}: 360.2691$, found: 360.2690 .

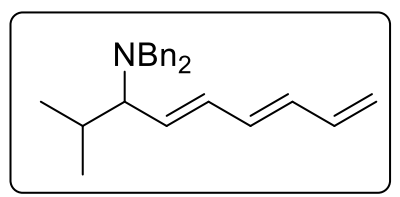

\section{$(3 E, 5 E)-N, N$-dibenzyl-8-methyl-nona-1,3,5-trien-7-amine (6ae)}

The title compound was prepared via general procedure A, purified by flash chromatography on silica gel eluting with in $\mathrm{CH}_{2} \mathrm{Cl}_{2} / \mathrm{PE}=1 / 3(\mathrm{Rf}=0.36)$, yellow oil, $62.4 \mathrm{mg}, 92 \%$ yield, ${ }^{1} \mathrm{H} \mathrm{NMR}\left(300 \mathrm{MHz}, \mathrm{CDCl}_{3}\right)$ $\delta 7.39(\mathrm{~d}, J=7.2 \mathrm{~Hz}, 4 \mathrm{H}), 7.34-7.26(\mathrm{~m}, 4 \mathrm{H}), 7.25-7.18(\mathrm{~m}, 2 \mathrm{H}), 6.41(\mathrm{dt}, J=16.8,10.2 \mathrm{~Hz}, 1 \mathrm{H}), 6.33-$ $6.18(\mathrm{~m}, 2 \mathrm{H}), 5.99(\mathrm{dd}, J=15.3,9.9 \mathrm{~Hz}, 1 \mathrm{H}), 5.64(\mathrm{dd}, J=15.3,10.2 \mathrm{~Hz}, 1 \mathrm{H}), 5.25(\mathrm{dd}, J=16.8,1.2 \mathrm{~Hz}$, $1 \mathrm{H}), 5.11(\mathrm{dd}, J=9.9,1.2 \mathrm{~Hz}, 1 \mathrm{H}), 3.85(\mathrm{~d}, J=13.8 \mathrm{~Hz}, 2 \mathrm{H}), 3.26(\mathrm{~d}, J=13.8 \mathrm{~Hz}, 2 \mathrm{H}), 2.52(\mathrm{t}, J=10.2$ $\mathrm{Hz}, 1 \mathrm{H}), 1.90-1.77(\mathrm{~m}, 1 \mathrm{H}), 1.08(\mathrm{~d}, J=6.6 \mathrm{~Hz}, 3 \mathrm{H}), 0.72(\mathrm{~d}, J=6.6 \mathrm{~Hz}, 3 \mathrm{H}) .{ }^{13} \mathrm{C} \mathrm{NMR}\left(75 \mathrm{MHz}, \mathrm{CDCl}_{3}\right)$ $\delta 140.5,137.1,134.5,132.9,132.6,132.5,128.9,128.3,126.8,117.3,67.5,53.9,29.6,21.0,20.5$. HRMS (ESI) calculated for $\mathrm{C}_{24} \mathrm{H}_{30} \mathrm{~N}[\mathrm{M}+\mathrm{H}]^{+}: 332.2378$, found: 332.2382 .

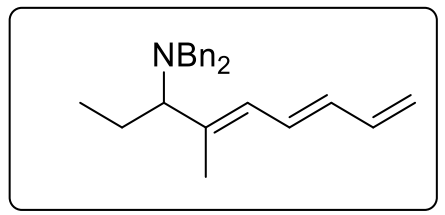

\section{$(3 E, 5 E)-N, N$-Dibenzyl-6-methyl-nona-1,3,5-trien-1-amine (6af)}

The title compound was prepared via general procedure A, purified by flash chromatography on silica gel eluting with in $\mathrm{CH}_{2} \mathrm{Cl}_{2} / \mathrm{PE}=1 / 3(\mathrm{Rf}=0.40)$, yellow oil, $58.1 \mathrm{mg}, 88 \%$ yield, ${ }^{1} \mathrm{H} \mathrm{NMR}\left(300 \mathrm{MHz}, \mathrm{CDCl}_{3}\right)$ $\delta$ 7.35-7.17 (m, 10H), 6.61-6.32 (m, 2H), $6.18(\mathrm{dd}, J=14.7,10.8 \mathrm{~Hz}, 1 \mathrm{H}), 5.86(\mathrm{~d}, J=10.8 \mathrm{~Hz}, 1 \mathrm{H}), 5.19$ $(\mathrm{d}, J=16.5 \mathrm{~Hz}, 1 \mathrm{H}), 5.06(\mathrm{~d}, J=10.2 \mathrm{~Hz}, 1 \mathrm{H}), 3.68(\mathrm{~d}, J=13.8 \mathrm{~Hz}, 2 \mathrm{H}), 3.51(\mathrm{~d}, J=13.8 \mathrm{~Hz}, 2 \mathrm{H}), 2.92$ 
$(\mathrm{dd}, J=9.6,4.8 \mathrm{~Hz}, 1 \mathrm{H}), 1.81\left(\mathrm{~d}, J=0.9 \mathrm{~Hz}, 3 \mathrm{H},-\mathrm{CH}_{3}\right) 1.84-1.71(\mathrm{~m}, 1 \mathrm{H}), 1.58-1.43(\mathrm{~m}, 1 \mathrm{H}), 0.80(\mathrm{t}, J$ $\left.=7.4 \mathrm{~Hz}, 3 \mathrm{H},-\mathrm{CH}_{3}\right) .{ }^{13} \mathrm{C} \mathrm{NMR}\left(75 \mathrm{MHz}, \mathrm{CDCl}_{3}\right) \delta 140.8,138.6,137.5,132.4,129.6,128.8,128.2,127.8$, 126.7, 116.6, 67.9, 54.4, 20.6, 15.5, 11.8. HRMS (ESI) calculated for $\mathrm{C}_{24} \mathrm{H}_{30} \mathrm{~N}[\mathrm{M}+\mathrm{H}]^{+}: 332.2378$, found: 332.2382 .

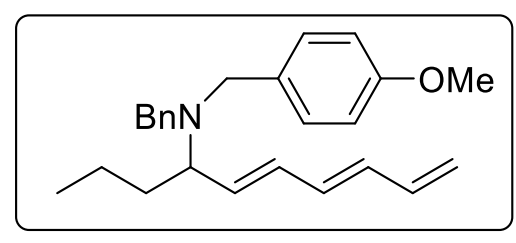

(3E,5E)-N-Benzyl- $N$-p-methoxylbenzyl-deca-1,3,5-trien-7-amine (6ag)

The title compound was prepared via general procedure A, purified by flash chromatography on silica gel eluting with in $\mathrm{CH}_{2} \mathrm{Cl}_{2} / \mathrm{PE}=1 / 1(\mathrm{Rf}=0.20)$, yellow oil, $54.1 \mathrm{mg}, 83 \%$ yield. ${ }^{1} \mathrm{H} \mathrm{NMR}\left(300 \mathrm{MHz}, \mathrm{CDCl}_{3}\right)$ $\delta$ 7.41-7.14 (m, 7H), $6.84(\mathrm{~d}, J=8.7 \mathrm{~Hz}, 2 \mathrm{H}), 6.45-6.32(\mathrm{~m}, 1 \mathrm{H}), 6.29-6.14(\mathrm{~m}, 2 \mathrm{H}), 6.05(\mathrm{dd}, J=15.3$, $9.3 \mathrm{~Hz}, 1 \mathrm{H}), 5.72(\mathrm{dd}, J=15.3,9.0 \mathrm{~Hz}, 1 \mathrm{H}), 5.23(\mathrm{dd}, J=16.8,1.5 \mathrm{~Hz}, 1 \mathrm{H}), 5.10$ (dd, $J=9.9,1.5 \mathrm{~Hz}$, 1H), $3.78(\mathrm{~s}, 3 \mathrm{H}), 3.77$ (d, $J=13.6 \mathrm{~Hz}, 1 \mathrm{H}), 3.72(\mathrm{~d}, J=13.6 \mathrm{~Hz}, 1 \mathrm{H}), 3.33$ (d, $J=13.2 \mathrm{~Hz}, 1 \mathrm{H}), 3.28$ (d, $J=13.2 \mathrm{~Hz}, 1 \mathrm{H}), 3.06(\mathrm{q}, J=7.2 \mathrm{~Hz}, 1 \mathrm{H}), 1.73-1.63(\mathrm{~m}, 1 \mathrm{H}), 1.45-1.20(\mathrm{~m}, 3 \mathrm{H}), 0.80(\mathrm{t}, J=7.2 \mathrm{~Hz}, 3 \mathrm{H})$. ${ }^{13} \mathrm{C} \mathrm{NMR}\left(75 \mathrm{MHz}, \mathrm{CDCl}_{3}\right) \delta 158.5,140.8,137.1,133.9,133.1,132.9,132.6,132.5,129.8,128.7,128.2$, $126.7,117.2,113.7,59.7,55.3,53.7,53.1,34.8,19.9$, 14.2. HRMS (ESI) calculated for $\mathrm{C}_{25} \mathrm{H}_{32} \mathrm{NO}$ $[\mathrm{M}+\mathrm{H}]^{+}:$326.2484, found: 326.2484 .

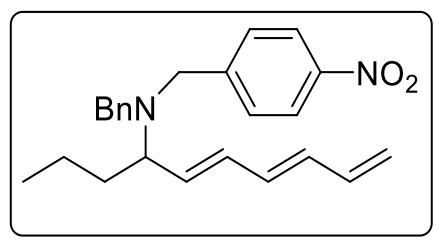

\section{(3E,5E)-N-Benzyl- $N$-p-nitrylbenzyl-deca-1,3,5-trien-7-amine (6ah)}

The title compound was prepared via general procedure A, purified by flash chromatography on silica gel eluting with in EtOAc/PE $=1 / 10(\mathrm{Rf}=0.40)$, yellow oil, $71.1 \mathrm{mg}, 94 \%$ yield, ${ }^{1} \mathrm{H} \mathrm{NMR}\left(400 \mathrm{MHz}, \mathrm{CDCl}_{3}\right)$ $\delta 8.15(\mathrm{~d}, J=8.8 \mathrm{~Hz}, 2 \mathrm{H}), 7.52(\mathrm{~d}, J=8.8 \mathrm{~Hz}, 2 \mathrm{H}), 7.36-7.29(\mathrm{~m}, 4 \mathrm{H}), 7.26-7.19(\mathrm{~m}, 1 \mathrm{H}), 6.40(\mathrm{dt}, J=$ $16.8,10.0 \mathrm{~Hz}, 1 \mathrm{H}), 6.32-6.16(\mathrm{~m}, 2 \mathrm{H}), 6.05(\mathrm{dd}, J=15.2,9.6 \mathrm{~Hz}, 1 \mathrm{H}), 5.70(\mathrm{dd}, J=15.2,9.2 \mathrm{~Hz}, 1 \mathrm{H})$, $5.25(\mathrm{dd}, J=16.6,1.2 \mathrm{~Hz}, 1 \mathrm{H}), 5.12(\mathrm{dd}, J=10.0,1.2 \mathrm{~Hz}, 1 \mathrm{H}), 3.81$ (d, $J=14.8 \mathrm{~Hz}, 1 \mathrm{H}), 3.79$ (d, $J=$ $13.6 \mathrm{~Hz}, 1 \mathrm{H}), 3.49(\mathrm{~d}, J=14.8 \mathrm{~Hz}, 1 \mathrm{H}), 3.40$ (d, $J=13.6 \mathrm{~Hz}, 1 \mathrm{H}), 3.02(\mathrm{q}, J=7.2 \mathrm{~Hz}, 1 \mathrm{H}), 1.80-1.61(\mathrm{~m}$, $1 \mathrm{H}), 1.52-1.22(\mathrm{~m}, 3 \mathrm{H}), 0.83(\mathrm{t}, J=7.2 \mathrm{~Hz}, 3 \mathrm{H}) .{ }^{13} \mathrm{C} \mathrm{NMR}\left(100 \mathrm{MHz}, \mathrm{CDCl}_{3}\right) \delta 148.8,147.1,139.8$, 136.9, 133.5, 133.1, 132.72, 132.67, 129.2, 128.7, 128.4, 127.1, 123.6, 117.7, 60.7, 54.1, 53.6, 34.9, 19.9, 14.2. HRMS (ESI) calculated for $\mathrm{C}_{24} \mathrm{H}_{29} \mathrm{~N}_{2} \mathrm{O}_{2}[\mathrm{M}+\mathrm{H}]^{+}: 337.2229$, found: 337.2226 .

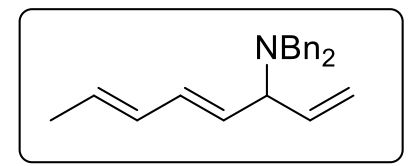

(4E,6E)- $N, N$-Dibenzylocta-1,4,6-trien-3-amine (7aa) 
The title compound was prepared via general procedure $\mathrm{B}$, purified by flash chromatography on silica gel eluting with in $\mathrm{CH}_{2} \mathrm{Cl}_{2} / \mathrm{PE}=1 / 2(\mathrm{Rf}=0.31)$, yellow oil, $46.1 \mathrm{mg}, 76 \%$ yield, ${ }^{1} \mathrm{H} \mathrm{NMR}\left(300 \mathrm{MHz}, \mathrm{CDCl}_{3}\right)$ $\delta$ 7.43-7.35 (m, 4H), 7.33-7.25 (m, 4H), 7.25-7.16 (m, 2H), 6.20-6.03 (m, 2H), 6.01-5.85 (m, 1H), 5.74$5.57(\mathrm{~m}, 2 \mathrm{H}), 5.25(\mathrm{dt}, J=10.5,1.8 \mathrm{~Hz}, 1 \mathrm{H}), 5.11(\mathrm{dt}, J=17.4,1.8 \mathrm{~Hz}, 1 \mathrm{H}), 3.71(\mathrm{t}, J=6.6 \mathrm{~Hz}, 1 \mathrm{H})$, 3.65-3.53 (m, 4H), $1.76(\mathrm{~d}, J=6.6 \mathrm{~Hz}, 3 \mathrm{H}) .{ }^{13} \mathrm{C} \mathrm{NMR}\left(75 \mathrm{MHz}, \mathrm{CDCl}_{3}\right) \delta 140.5,137.9,133.7,131.4$, 129.6, 129.1, 128.7, 128.3, 126.8, 117.7, 62.7, 53.9, 18.2. HRMS (ESI) calculated for $\mathrm{C}_{22} \mathrm{H}_{26} \mathrm{~N}[\mathrm{M}+\mathrm{H}]^{+}$: 304.2065, found: 304.2066 .

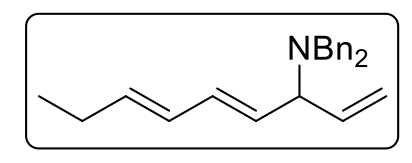

(4E,6E)-N,N-dibenzylnona-1,4,6-trien-3-amine (7ab)

The title compound was prepared via general procedure B, purified by flash chromatography on silica gel eluting with in $\mathrm{CH}_{2} \mathrm{Cl}_{2} / \mathrm{PE}=1 / 3(\mathrm{Rf}=0.27)$, yellow oil, $50.1 \mathrm{mg}, 79 \%$ yield, ${ }^{1} \mathrm{H} \mathrm{NMR}\left(300 \mathrm{MHz}, \mathrm{CDCl}_{3}\right)$ $\delta 7.39(\mathrm{~d}, J=7.2 \mathrm{~Hz}, 4 \mathrm{H}), 7.33-7.27(\mathrm{~m}, 4 \mathrm{H}), 7.24-7.18(\mathrm{~m}, 2 \mathrm{H}), 6.14-6.03(\mathrm{~m}, 2 \mathrm{H}), 5.95-5.86(\mathrm{~m}, 1 \mathrm{H})$, 5.77-5.61 (m, 2H), $5.26(\mathrm{dt}, J=10.5,1.5 \mathrm{~Hz}, 1 \mathrm{H}), 5.11(\mathrm{dt}, J=17.4,1.5 \mathrm{~Hz}, 1 \mathrm{H}), 3.71(\mathrm{t}, J=6.3 \mathrm{~Hz}, 1 \mathrm{H})$, $3.60(\mathrm{~s}, 4 \mathrm{H}), 2.21-1.99(\mathrm{~m}, 2 \mathrm{H}), 1.02(\mathrm{t}, J=7.2 \mathrm{~Hz}, 3 \mathrm{H}) .{ }^{13} \mathrm{C} \mathrm{NMR}\left(75 \mathrm{MHz}, \mathrm{CDCl}_{3}\right) \delta 140.5,137.9$, 136.2, 133.8, 129.9, 129.0, 128.7, 128.3, 126.8, 117.8, 62.8, 53.9, 25.8, 13.6. HRMS (ESI) calculated for $\mathrm{C}_{23} \mathrm{H}_{28} \mathrm{~N}[\mathrm{M}+\mathrm{H}]^{+}:$318.2222, found: 318.2220 .

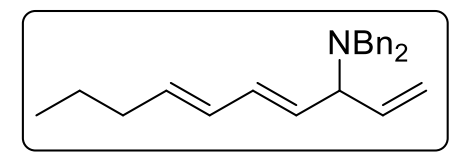

(4E,6E)- $N, N$-Dibenzyldeca-1,4,6-trien-3-amine (7ac)

The title compound was prepared via general procedure B, purified by flash chromatography on silica gel eluting with in $\mathrm{CH}_{2} \mathrm{Cl}_{2} / \mathrm{PE}=1 / 3(\mathrm{Rf}=0.22)$, yellow oil, $57.1 \mathrm{mg}, 86 \%$ yield, ${ }^{1} \mathrm{H} \mathrm{NMR}\left(300 \mathrm{MHz}, \mathrm{CDCl}_{3}\right)$ $\delta 7.38(\mathrm{~d}, J=7.2 \mathrm{~Hz}, 4 \mathrm{H}), 7.29(\mathrm{t}, J=7.2 \mathrm{~Hz}, 4 \mathrm{H}), 7.22-7.16(\mathrm{~m}, 2 \mathrm{H}), 6.11-6.02(\mathrm{~m}, 2 \mathrm{H}), 6.01-5.88(\mathrm{~m}$, $1 \mathrm{H}), 5.71-5.63(\mathrm{~m}, 2 \mathrm{H}), 5.25(\mathrm{dt}, J=10.5,1.5 \mathrm{~Hz}, 1 \mathrm{H}), 5.11(\mathrm{dt}, J=17.1,1.5 \mathrm{~Hz}, 1 \mathrm{H}), 3.71(\mathrm{t}, J=6.3$ $\mathrm{Hz}, 1 \mathrm{H}), 3.60(\mathrm{~s}, 4 \mathrm{H}), 2.06(\mathrm{q}, J=7.2 \mathrm{~Hz}, 2 \mathrm{H}), 1.45-1.38(\mathrm{~m}, 2 \mathrm{H}), 0.91(\mathrm{t}, J=7.5 \mathrm{~Hz}, 3 \mathrm{H}) .{ }^{13} \mathrm{C} \mathrm{NMR}(75$ $\left.\mathrm{MHz}, \mathrm{CDCl}_{3}\right) \delta 140.5,137.8,134.5,133.8,130.1,130.0,128.7,128.3,126.8,117.8,62.8,53.9,34.9$, 22.6, 13.9. HRMS (ESI) calculated for $\mathrm{C}_{24} \mathrm{H}_{30} \mathrm{~N}[\mathrm{M}+\mathrm{H}]^{+}: 332.2378$, found: 332.2382 .

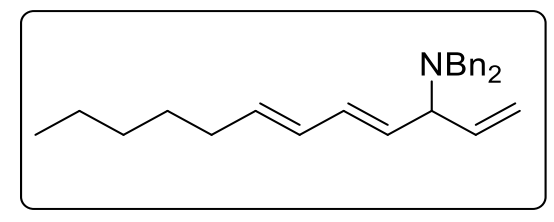

\section{(4E,6E)- $N, N$-Dibenzyl-dodeca-1,4,6-trien-3-amine (7ad)}

The title compound was prepared via general procedure $\mathrm{B}$, purified by flash chromatography on silica gel eluting with in $\mathrm{CH}_{2} \mathrm{Cl}_{2} / \mathrm{PE}=1 / 3(\mathrm{Rf}=0.26)$, yellow oil, $51.4 \mathrm{mg}, 72 \%$ yield, ${ }^{1} \mathrm{H} \mathrm{NMR}\left(400 \mathrm{MHz}, \mathrm{CDCl}_{3}\right)$ $\delta 7.38(\mathrm{~d}, J=7.2 \mathrm{~Hz}, 4 \mathrm{H}), 7.29$ (t, $J=7.2 \mathrm{~Hz}, 4 \mathrm{H}), 7.20$ (t, $J=7.2 \mathrm{~Hz}, 2 \mathrm{H}), 6.15-6.02(\mathrm{~m}, 2 \mathrm{H}), 5.94$ (ddd, 
$J=17.1,10.4,6.4 \mathrm{~Hz}, 1 \mathrm{H}), 5.66(\mathrm{dt}, J=13.6,6.7 \mathrm{~Hz}, 2 \mathrm{H}), 5.25(\mathrm{~d}, J=10.4 \mathrm{~Hz}, 1 \mathrm{H}), 5.11(\mathrm{~d}, J=17.4$ $\mathrm{Hz}, 1 \mathrm{H}), 3.71(\mathrm{t}, J=6.2 \mathrm{~Hz}, 1 \mathrm{H}), 3.63-3.52$ (m, 4H), 2.08 (q, $J=7.1 \mathrm{~Hz}, 2 \mathrm{H}), 1.44-1.36(\mathrm{~m}, 2 \mathrm{H}), 1.33-$ $1.23(\mathrm{~m}, 4 \mathrm{H}), 0.89$ (t, $J=6.7 \mathrm{~Hz}, 3 \mathrm{H}) .{ }^{13} \mathrm{C} \mathrm{NMR}\left(100 \mathrm{MHz}, \mathrm{CDCl}_{3}\right) \delta 140.5,137.8,134.8,133.8,130.0$, $129.9,128.7,128.3,126.8,117.8,62.8,53.9,32.8,31.6,29.1,22.7,14.2$. HRMS (ESI) calculated for $\mathrm{C}_{26} \mathrm{H}_{34} \mathrm{~N}[\mathrm{M}+\mathrm{H}]^{+}:$360.2691, found: 360.2697 .

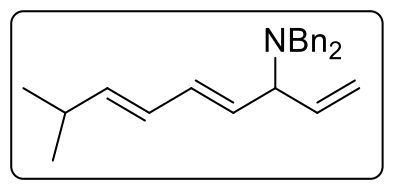

(4E,6E)- $N, N$-dibenzyl-8-methyl-nona-1,4,6-trien-3-amine (7ae)

The title compound was prepared via general procedure B, purified by flash chromatography on silica gel eluting with in $\mathrm{CH}_{2} \mathrm{Cl}_{2} / \mathrm{PE}=1 / 3(\mathrm{Rf}=0.25)$, yellow oil, $55.8 \mathrm{mg}, 84 \%$ yield, ${ }^{1} \mathrm{H} \mathrm{NMR}\left(300 \mathrm{MHz}, \mathrm{CDCl}_{3}\right)$ $\delta 7.39(\mathrm{~d}, J=7.0 \mathrm{~Hz}, 4 \mathrm{H}), 7.33-7.27$ (m, 4H), $7.24-7.16$ (m, 2H), 6.13-6.00 (m, 2H), 5.94 (ddd, $J=17.2$, $10.4,6.4 \mathrm{~Hz}, 1 \mathrm{H}), 5.74-5.60(\mathrm{~m}, 2 \mathrm{H}), 5.26(\mathrm{dt}, J=10.4,1.6 \mathrm{~Hz}, 1 \mathrm{H}), 5.11(\mathrm{dt}, J=17.4,1.6 \mathrm{~Hz}, 1 \mathrm{H}), 3.71$ (t, $J=6.3 \mathrm{~Hz}, 1 \mathrm{H}), 3.59$ (d, $J=9.4 \mathrm{~Hz}, 4 \mathrm{H}), 2.34(\mathrm{dq}, J=13.3,6.7 \mathrm{~Hz}, 1 \mathrm{H}), 1.02(\mathrm{~d}, J=6.7 \mathrm{~Hz}, 3 \mathrm{H})$, $1.01(\mathrm{~d}, J=6.7 \mathrm{~Hz}, 3 \mathrm{H}) .{ }^{13} \mathrm{C} \mathrm{NMR}\left(75 \mathrm{MHz}, \mathrm{CDCl}_{3}\right) \delta 141.6,140.5,137.8,133.9,130.2,128.7,128.3$, 127.0, 126.8, 117.8, 62.8, 53.9, 31.2, 22.5, 22.4. HRMS (ESI) calculated for $\mathrm{C}_{24} \mathrm{H}_{30} \mathrm{~N}[\mathrm{M}+\mathrm{H}]^{+}: 332.2378$, found: 332.2382 .

\section{Control experiment synthesis of 5ak.}

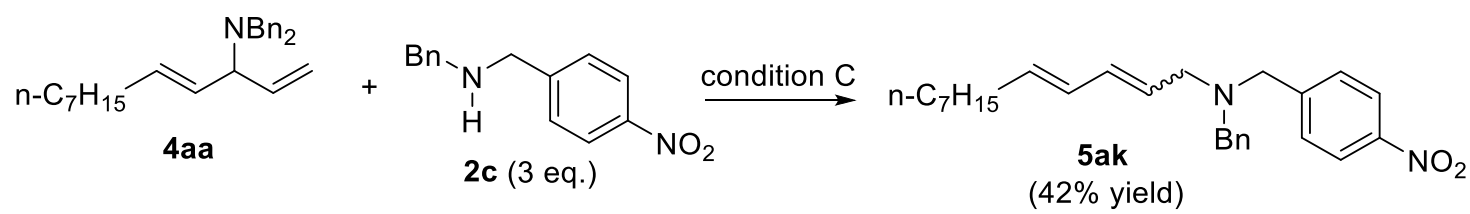

A flame dried tube was cooled to $\mathrm{rt}$. and charged with $\left[\operatorname{Ir}(\operatorname{cod}) \mathrm{Cl}_{2}(2.6 \mathrm{mg}, 4 \mu \mathrm{mol}, 4 \mathrm{~mol} \%)\right.$ and ligand $\mathbf{L 1}(8.0 \mathrm{mg}, 16 \mu \mathrm{mol}, 16 \mathrm{~mol} \%)$. The tube backfilled with $\mathrm{N}_{2}$. The flask was added freshly distilled toluene $(2.0 \mathrm{~mL})$, and stirred for $15 \mathrm{~min}$. Then the $4 \mathbf{a a}(0.1 \mathrm{mmol}, 1.0$ equiv), secondary amines $\mathbf{2 c}(0.4$ mmol, 4.0 equiv.), and $\mathrm{Sc}(\mathrm{OTf})_{3}(50 \mathrm{mg}, 0.1 \mathrm{mmol}, 100 \mathrm{~mol} \%)$ were added. The tube was sealed and stirred at $80^{\circ} \mathrm{C}$ for $24 \mathrm{~h}$. The solution was quenched with water $(10 \mathrm{~mL})$ and extracted with EA $(3 \times 10$ $\mathrm{mL}$ ). The organic layers dried over $\mathrm{Na}_{2} \mathrm{SO}_{4}$ and concentrated by rotary evaporation. The crude product was purified by silica gel column chromatography $(\mathrm{EtOAc} / \mathrm{PE}=1 / 20, \mathrm{Rf}=0.16)$ to afford the 5ak, yellow oil, E,E/E,Z = 5/1, $48.2 \mathrm{mg}, 42 \%$ yield, E,E-isomer: ${ }^{1} \mathrm{H}$ NMR (400 MHz, $\left.\mathrm{CDCl}_{3}\right) \delta 8.16(\mathrm{~d}, J=8.8 \mathrm{~Hz}$, 2H), 7.53 (d, $J=8.8 \mathrm{~Hz}, 2 \mathrm{H}), 7.39-7.29(\mathrm{~m}, 4 \mathrm{H}), 7.25-7.20(\mathrm{~m}, 1 \mathrm{H}), 6.17-6.00(\mathrm{~m}, 2 \mathrm{H})$, 5.72-5.60 (m, 2H), 3.69-3.46 (m, 4H), $3.08(\mathrm{~d}, J=6.8 \mathrm{~Hz}, 2 \mathrm{H}), 2.06$ (q, $J=7.2 \mathrm{~Hz}, 2 \mathrm{H}), 1.41-1.21(\mathrm{~m}, 10 \mathrm{H}), 0.87(\mathrm{t}, J$ $=6.8 \mathrm{~Hz}, 3 \mathrm{H}) .{ }^{13} \mathrm{C} \mathrm{NMR}\left(100 \mathrm{MHz}, \mathrm{CDCl}_{3}\right) \delta 148.2,147.1,139.1,135.0,133.9,129.6,129.3,128.8$, $128.5,127.7,127.2$, 123.6, 58.3, 57.2, 55.9, 32.8, 31.9, 29.4, 29.33, 29.31, 22.8, 14.2. HRMS (ESI) calculated for $\mathrm{C}_{26} \mathrm{H}_{35} \mathrm{~N}_{2} \mathrm{O}_{2}[\mathrm{M}+\mathrm{H}]^{+}: 407.2699$, found: 407.2700. 


\section{Preparation for 8.}

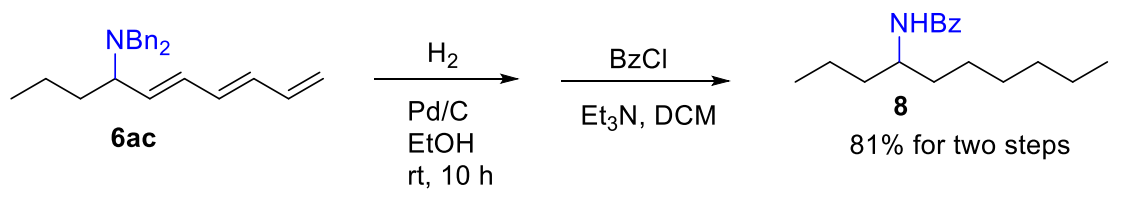

To a solution of $6 \mathbf{a c}(66 \mathrm{mg}, 0.2 \mathrm{mmol})$ in $\mathrm{EtOH}(5 \mathrm{~mL})$ was added $\mathrm{Pd} / \mathrm{C}(6.6 \mathrm{mg})$, then reaction were exchanged fullfill hydrogen with a balloon, and stirred at room temperature for $10 \mathrm{~h}$. After the reaction completed, the $\mathrm{Pd} / \mathrm{C}$ complex were filtered, and the reaction solution was then removed in vacuo give the crude amine.

The crude amine and $\mathrm{BzCl}(0.25 \mathrm{mmol})$ was dissolved in $\mathrm{CH}_{2} \mathrm{Cl}_{2}(5 \mathrm{~mL})$ and stirred for $10 \mathrm{~min}$, and $\mathrm{Et}_{3} \mathrm{~N}$ was added dropwise. This mixture was stirred at $\mathrm{rt}$ for $2 \mathrm{~h}$ and and sat $\mathrm{NH}_{4} \mathrm{Cl}(5 \mathrm{~mL})$ was added. The aqueous layer was extracted with EtOAc $(3 * 10 \mathrm{~mL})$ and the combined organic extracts were dried $\mathrm{Na}_{2} \mathrm{SO}_{4}$ and concentrated in vacuo. The residue was purified by column chromatography using hexanes/ethyl acetate $(10 / 1, \mathrm{Rf}=0.35)$ as the eluent to yield $\mathbf{8}$ in $81 \%$ (two steps).

8. ${ }^{1} \mathrm{H}$ NMR $\left(300 \mathrm{MHz}, \mathrm{CDCl}_{3}\right) \delta$ 7.81-7.68 (m, 2H), 7.52-7.35 (m, 3H), 5.87 (d, J=8.6 Hz, 1H), 4.19$4.08(\mathrm{~m}, 1 \mathrm{H}), 1.65-1.23(\mathrm{~m}, 14 \mathrm{H}), 0.93(\mathrm{t}, J=7.2 \mathrm{~Hz}, 3 \mathrm{H}), 0.87(\mathrm{t}, J=6.9 \mathrm{~Hz}, 3 \mathrm{H}) .{ }^{13} \mathrm{C} \mathrm{NMR}(75 \mathrm{MHz}$, $\left.\mathrm{CDCl}_{3}\right) \delta 167.2,135.3,131.3,128.6,126.9,49.7,37.7,35.5,31.9,29.4,26.0,22.7,19.3,14.21,14.19$. HRMS (ESI) calculated for $\mathrm{C}_{17} \mathrm{H}_{28} \mathrm{NO}[\mathrm{M}+\mathrm{H}]^{+}:$262.2171, found: 262.2170.

\section{Preparation for 9.}

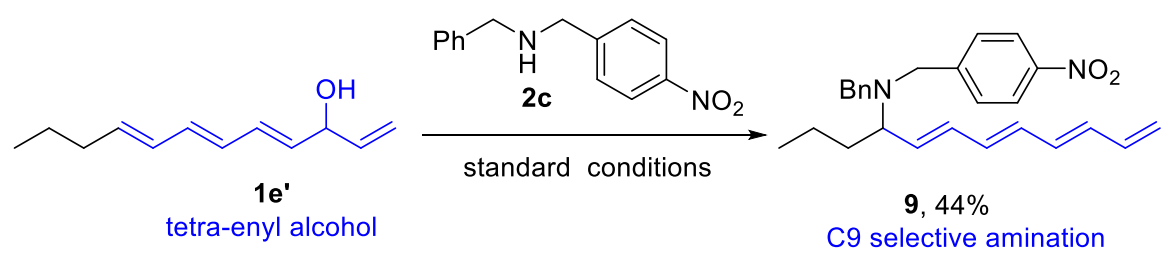

A flame dried tube was cooled to rt. and charged with $[\operatorname{Ir}(\operatorname{cod}) \mathrm{Cl}]_{2}(5.2 \mathrm{mg}, 8 \mu \mathrm{mol}, 4 \mathrm{~mol} \%)$ and ligand $\mathbf{L 1}(16.0 \mathrm{mg}, 32 \mu \mathrm{mol}, 16 \mathrm{~mol} \%)$. The tube backfilled with $\mathrm{N}_{2}$. The flask was added freshly distilled DCM (2.0 mL), and stirred for $15 \mathrm{~min}$. Then 1e' ( $0.2 \mathrm{mmol}, 1.0$ equiv), $2 \mathbf{c}(0.8 \mathrm{mmol}, 4.0$ equiv), and $\mathrm{Sc}(\mathrm{OTf})_{3}(98 \mathrm{mg}, 0.2 \mathrm{mmol}, 100 \mathrm{~mol} \%$ ) were added. The tube was sealed and stirred at rt. After the reaction was complete (monitored by TLC), the solution was quenched with water $(10 \mathrm{~mL})$ and extracted with DCM $(3 * 10 \mathrm{~mL})$. The organic layers dried over $\mathrm{Na}_{2} \mathrm{SO}_{4}$ and concentrated by rotary evaporation. The crude product was purified by silica gel column chromatography $(\mathrm{DCM} / \mathrm{PE}=1 / 3, \mathrm{Rf}=0.15)$ to afford the desired product 9. yellow oil, $35.5 \mathrm{mg}, 44 \%$ yield, ${ }^{1} \mathrm{H}$ NMR (400 MHz, CDCl3) $\delta 8.18(\mathrm{~d}, J=8.4 \mathrm{~Hz}$, 2H), $7.54(\mathrm{~d}, J=8.4 \mathrm{~Hz}, 2 \mathrm{H}), 7.44-7.28(\mathrm{~m}, 4 \mathrm{H}), 7.27-7.24(\mathrm{~m}, 1 \mathrm{H}), 6.51-6.37(\mathrm{~m}, 1 \mathrm{H}), 6.36-6.20$ (m, 4H), 6.09 (dd, $J=15.2,9.2 \mathrm{~Hz}, 1 \mathrm{H}), 5.72(\mathrm{dd}, J=15.2,9.2 \mathrm{~Hz}, 1 \mathrm{H}), 5.27$ (d, $J=16.4 \mathrm{~Hz}, 1 \mathrm{H}), 5.14(\mathrm{~d}, J$ $=10.8 \mathrm{~Hz}, 1 \mathrm{H}), 3.83(\mathrm{~d}, J=14.4 \mathrm{~Hz}, 1 \mathrm{H}), 3.81(\mathrm{~d}, J=13.2 \mathrm{~Hz}, 1 \mathrm{H}), 3.52(\mathrm{~d}, \mathrm{~J}=14.4 \mathrm{~Hz}, 1 \mathrm{H}), 3.42(\mathrm{~d}, J$ 
$=13.2 \mathrm{~Hz}, 1 \mathrm{H}), 3.04(\mathrm{q}, J=7.2 \mathrm{~Hz}, 1 \mathrm{H}), 1.77-1.64(\mathrm{~m}, 1 \mathrm{H}), 1.54-1.26(\mathrm{~m}, 3 \mathrm{H}), 0.85(\mathrm{t}, J=7.2 \mathrm{~Hz}, 3 \mathrm{H})$. ${ }^{13} \mathrm{C}$ NMR $(75 \mathrm{MHz}, \mathrm{CDCl} 3) \delta 148.9,147.1,139.9,137.1,133.8,133.8,133.2,132.9,132.6,132.5,129.3$, $128.8,128.5,127.2,123.6,117.6,60.8,54.1,53.7,34.9,19.9,14.2$. HRMS (ESI) calculated for $\mathrm{C}_{26} \mathrm{H}_{31} \mathrm{~N}_{2} \mathrm{O}_{2}[\mathrm{M}+\mathrm{H}]^{+}:$403.2386, found: 403.2388 .

\section{References}

(1) (a) Veits, G. K.; Carter, K. K.; Cox, S. J.; McNeil, A. J. J. Am. Chem. Soc. 2016, 138,12228. (b) Gaeta, C.; Talottaa, C.; Neri, P. Chem. Commun. 2014, 50, 9917.

[2] (a) Lipowsky, G.; Helmchen, G. Chem. Commun. 2004, 116. (b) Zheng, W.-H.; Sun, N.; Hou, X.-L. Org. Lett. 2005, 7, 5151. (c) Li, P.-F.; Wang, H.-L; Qu, J. J. Org. Chem. 2014, 79, 3955.

[3] Qiao, C.; Chen, A.; Gao, B.; Liu, Y.; Huang, H. Chin. J. Chem. 2018, 36, 929. 


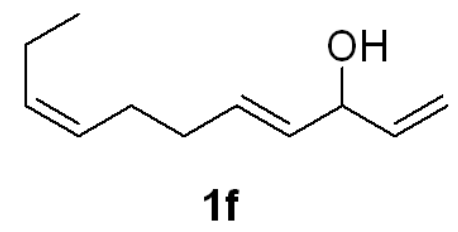

${ }^{1} \mathrm{H} \mathrm{NMR}, 400 \mathrm{MHz}, \mathrm{CDCl}_{3}$

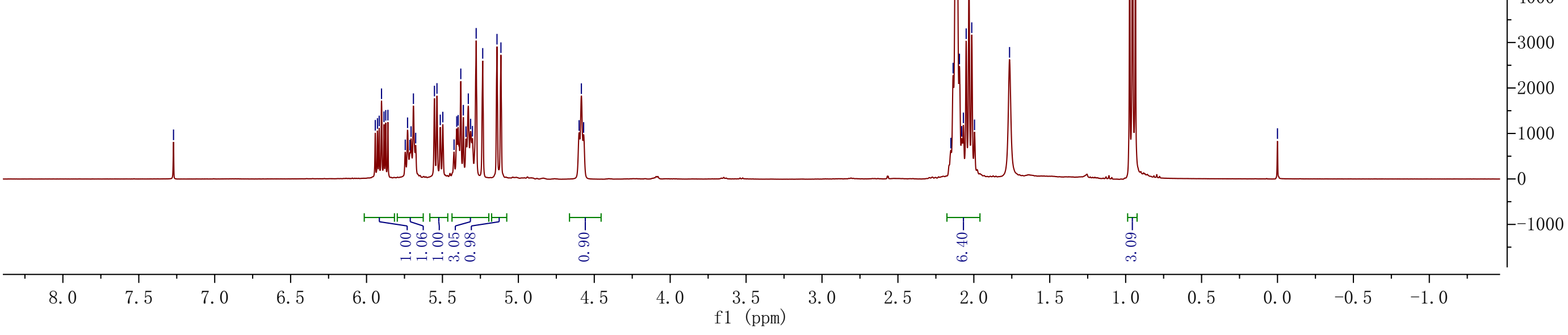




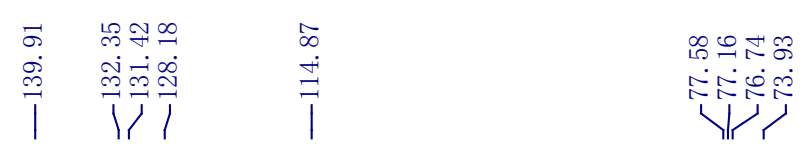

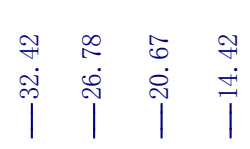

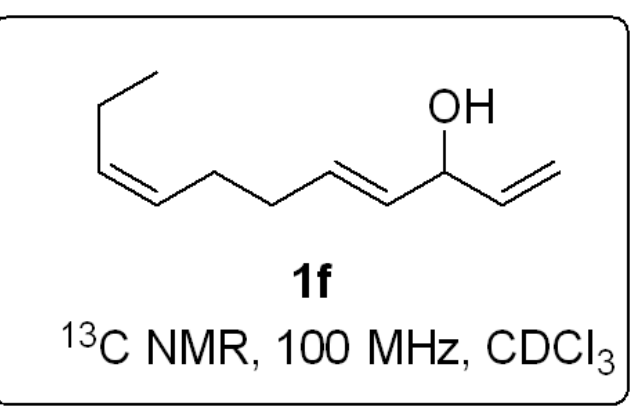




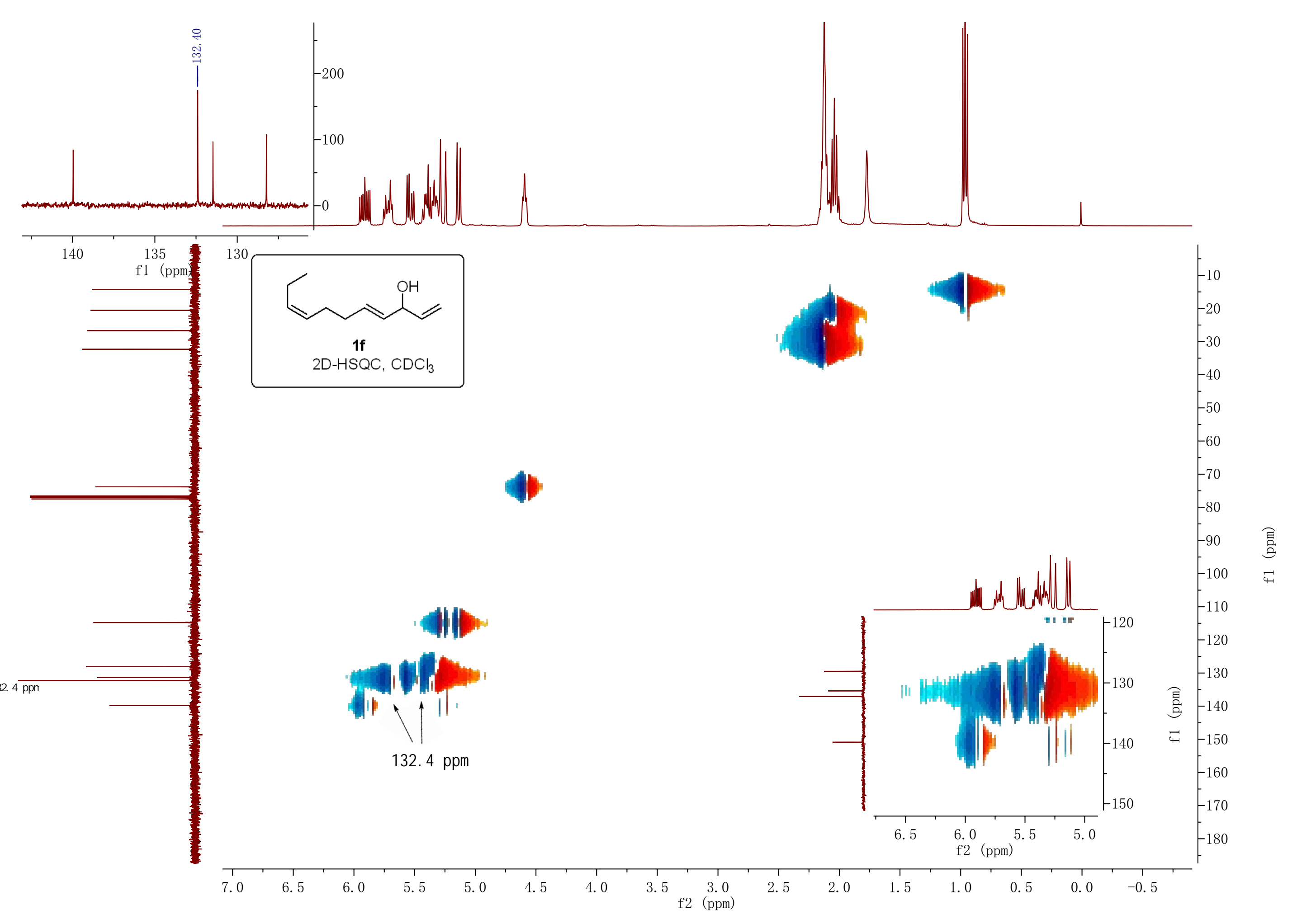



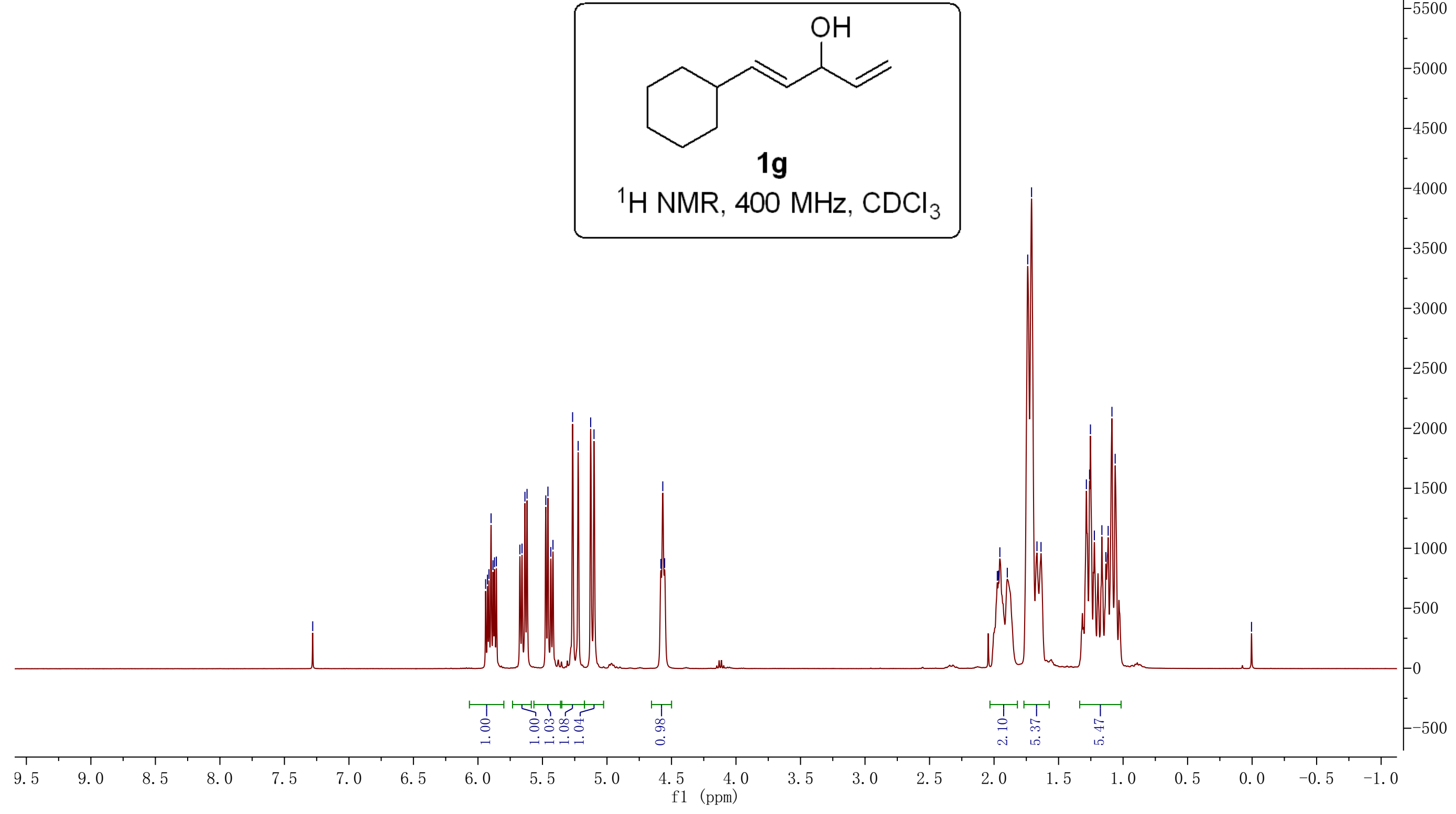

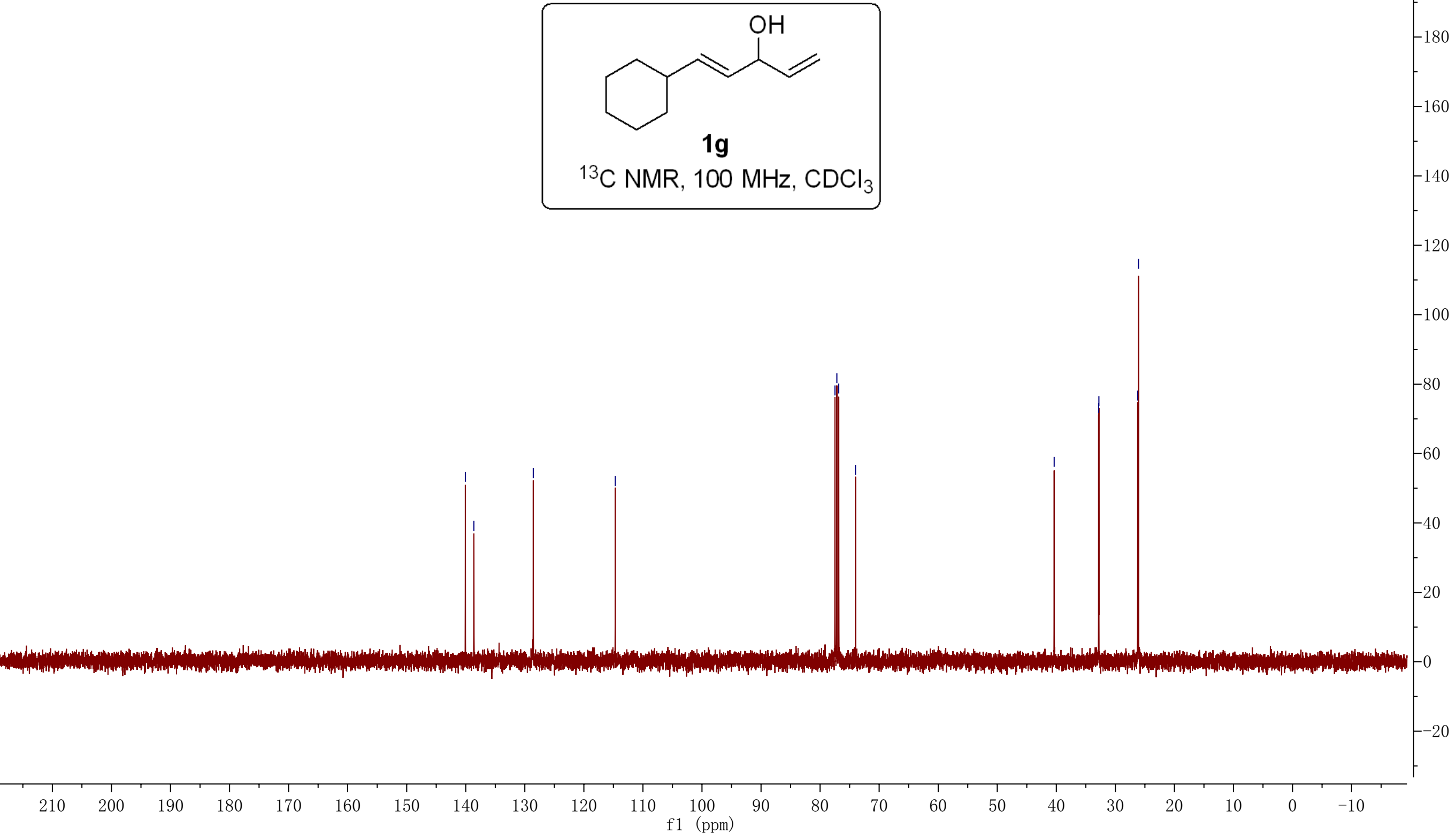


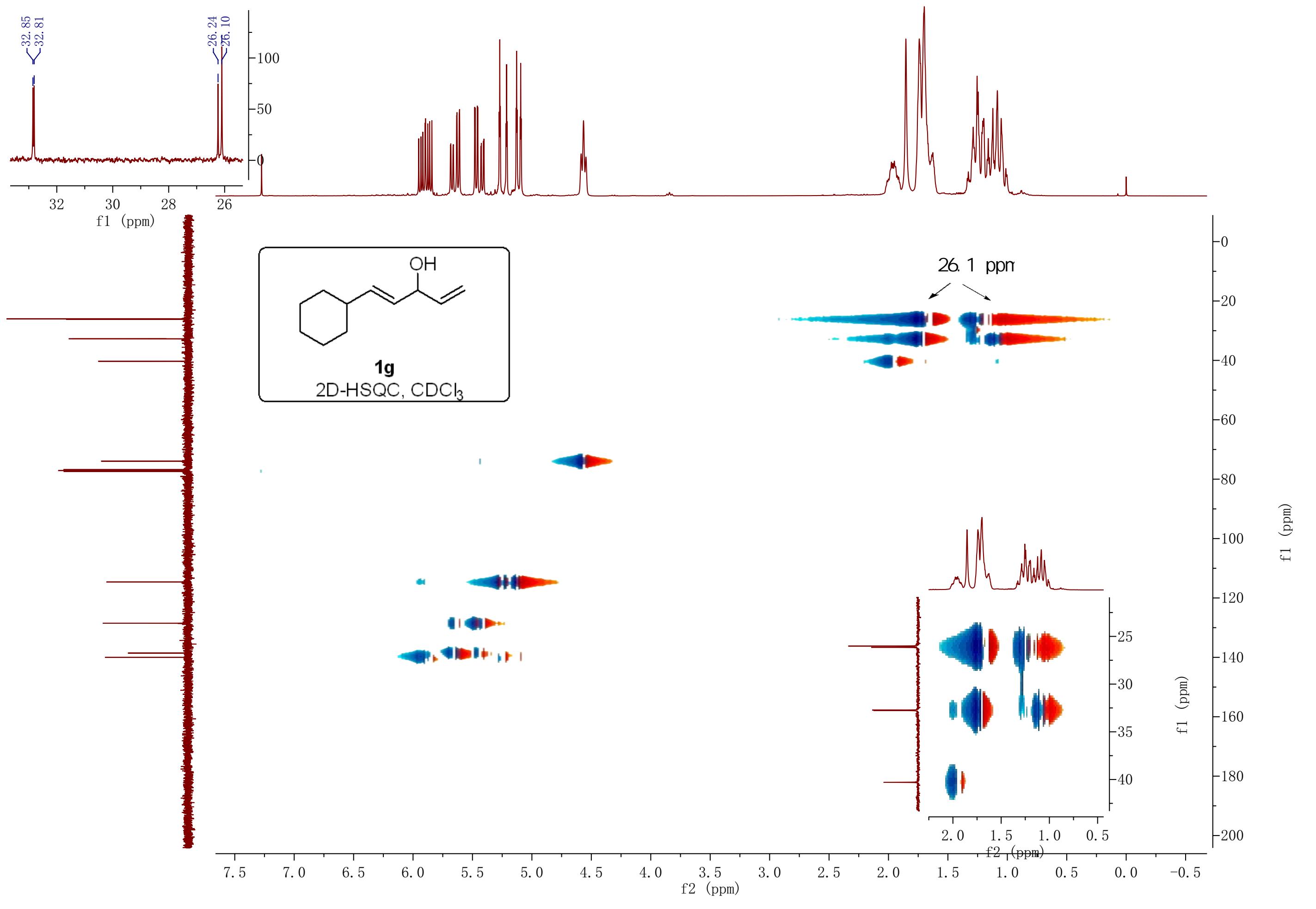



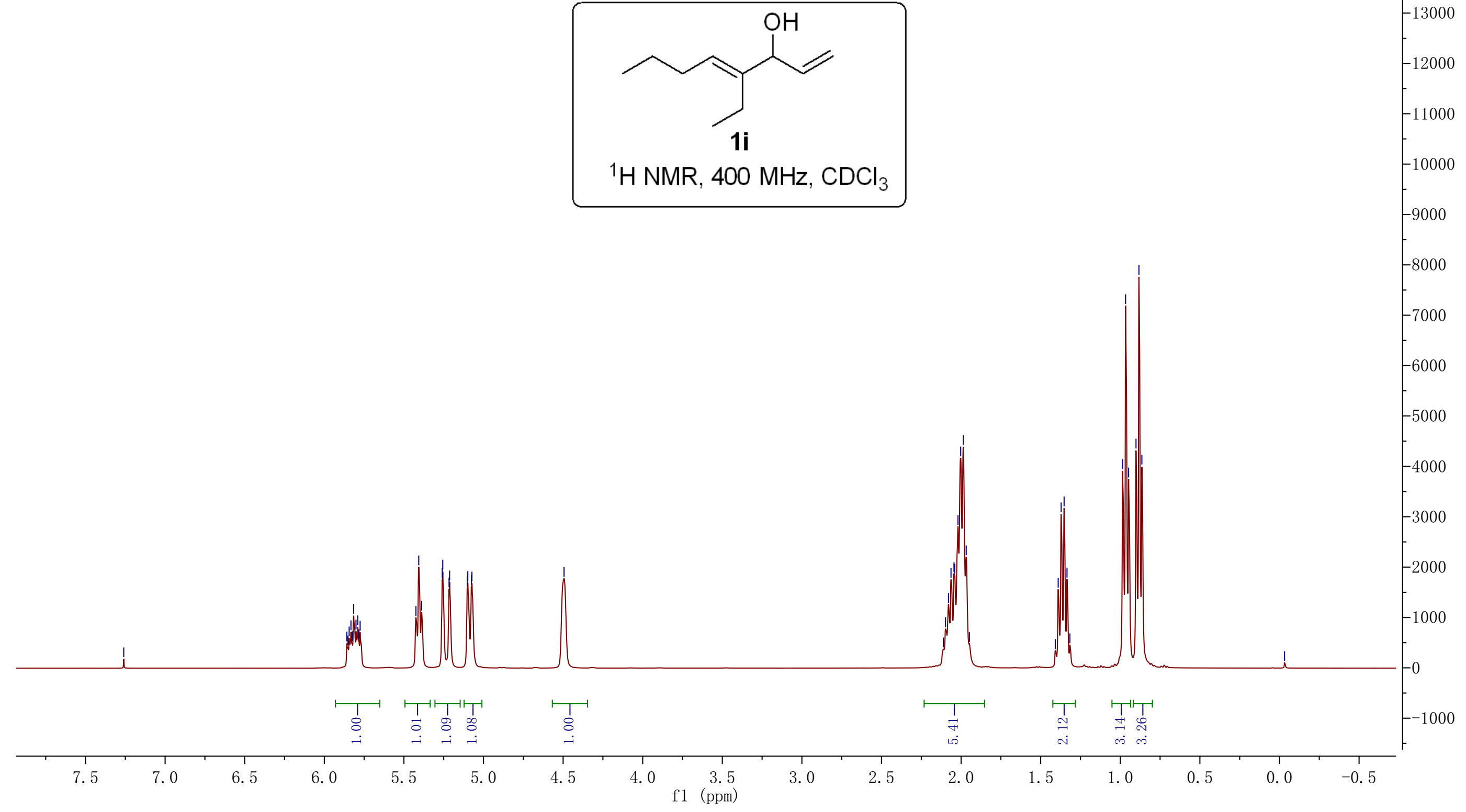

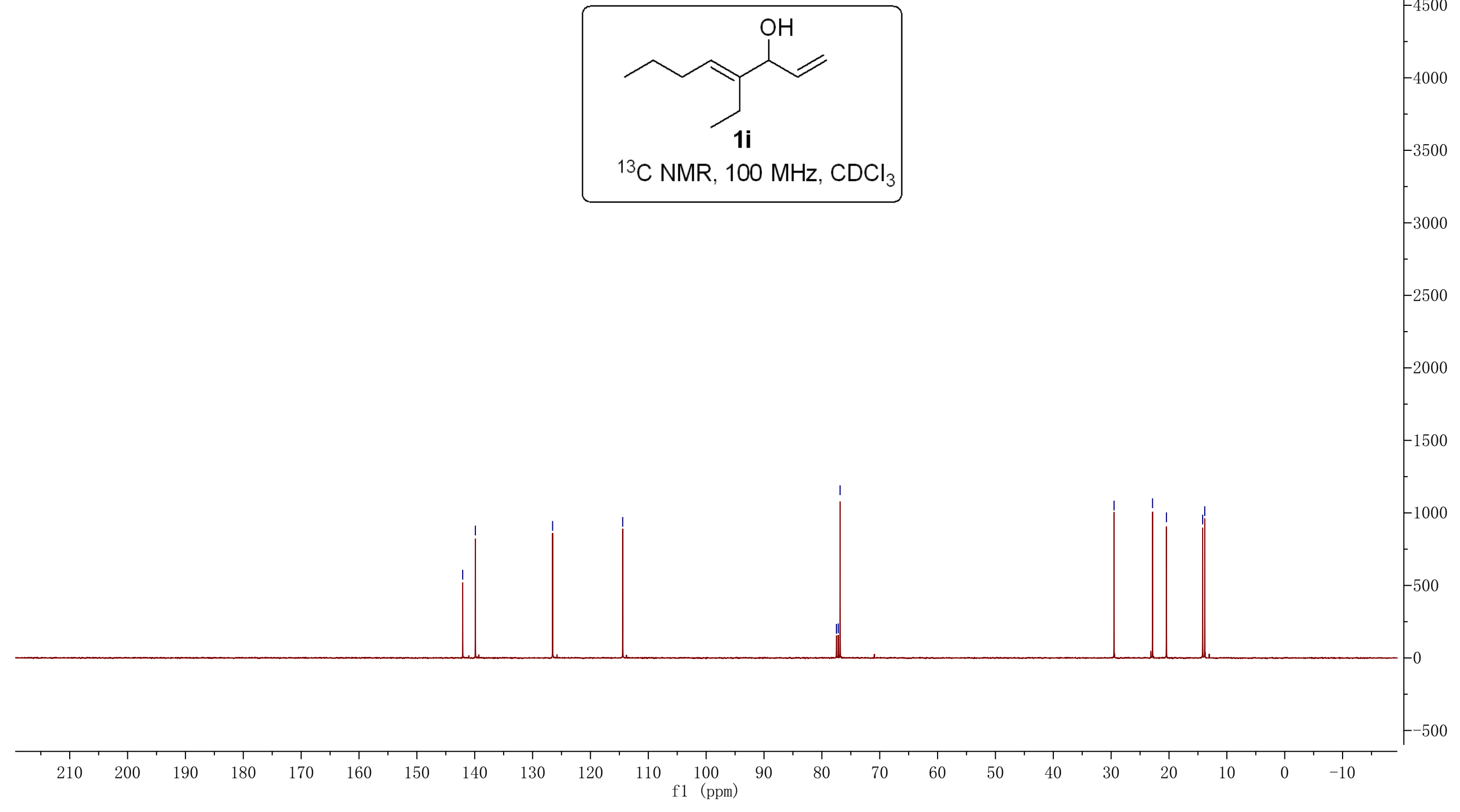

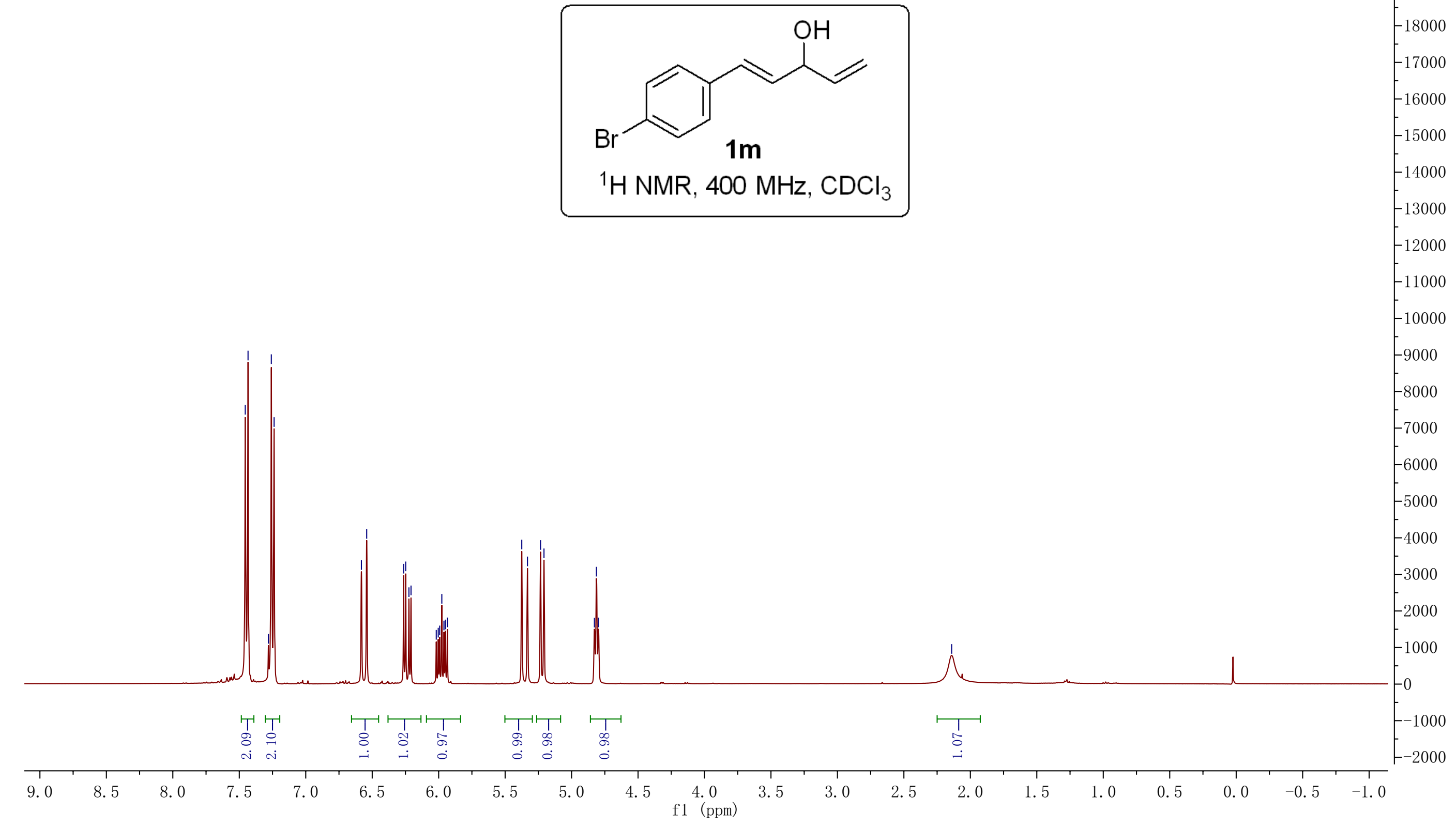


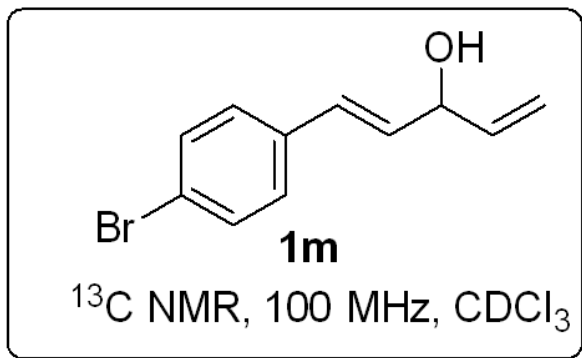

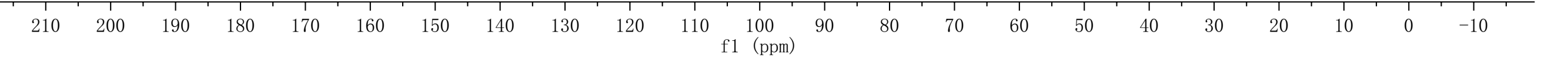



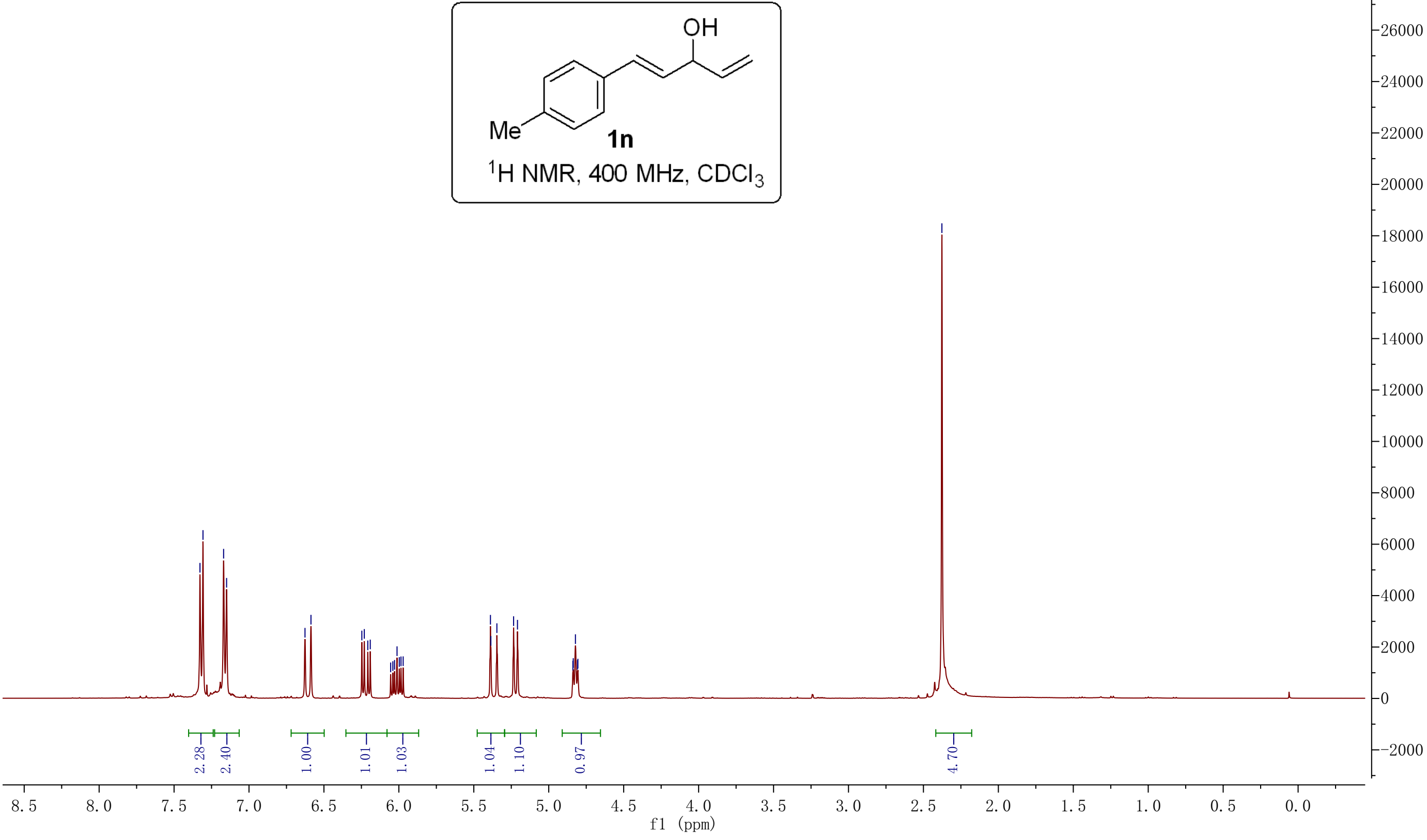


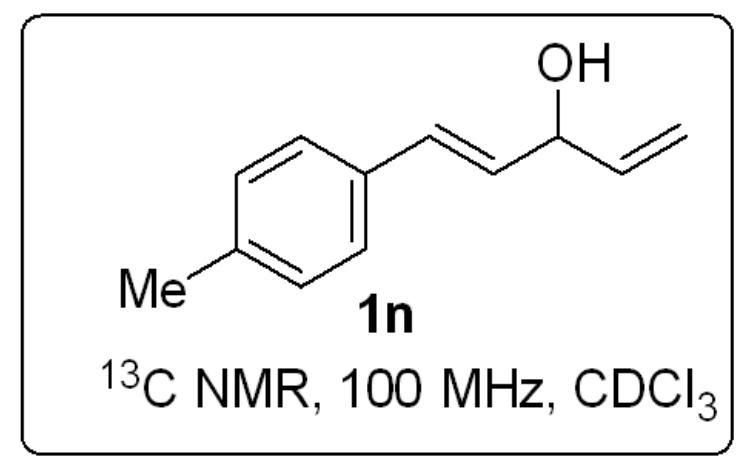




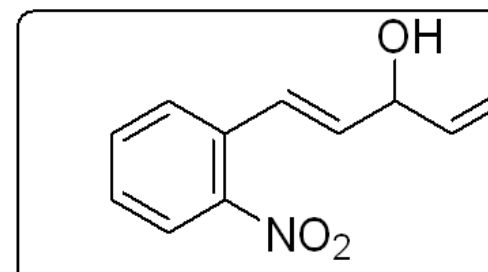

$1 q$

${ }^{13} \mathrm{C}$ NMR, $100 \mathrm{MHz}, \mathrm{CDCl}_{3}$

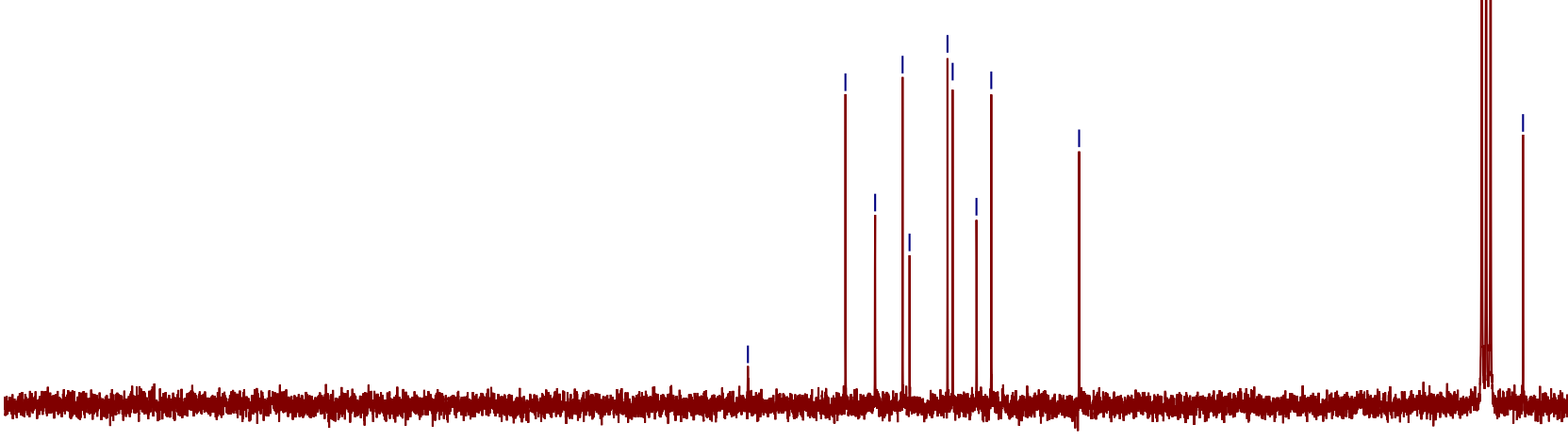




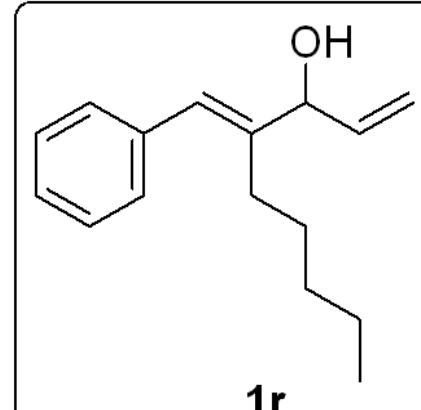

${ }^{1} \mathrm{H} \mathrm{NMR}, 400 \mathrm{MHz}, \mathrm{CDCl}_{3}$

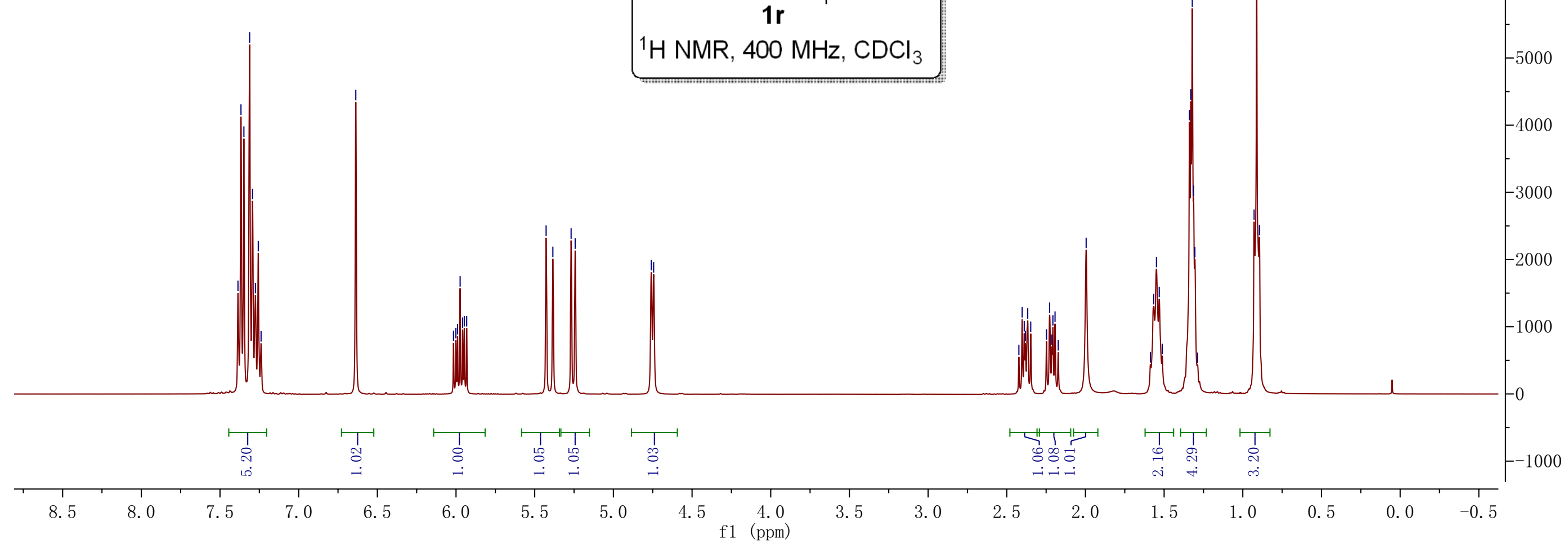




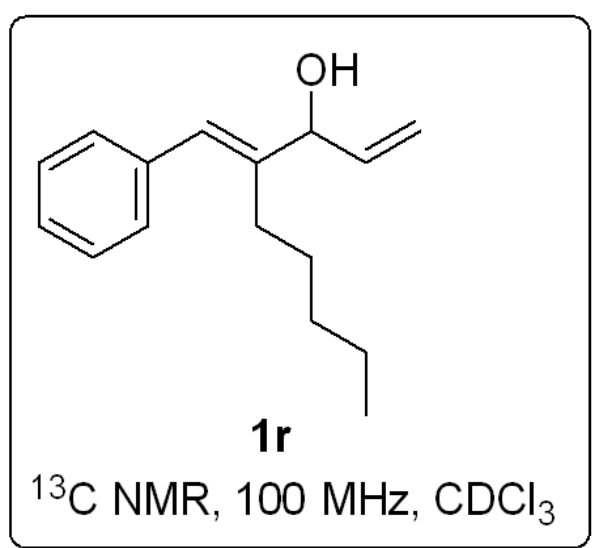



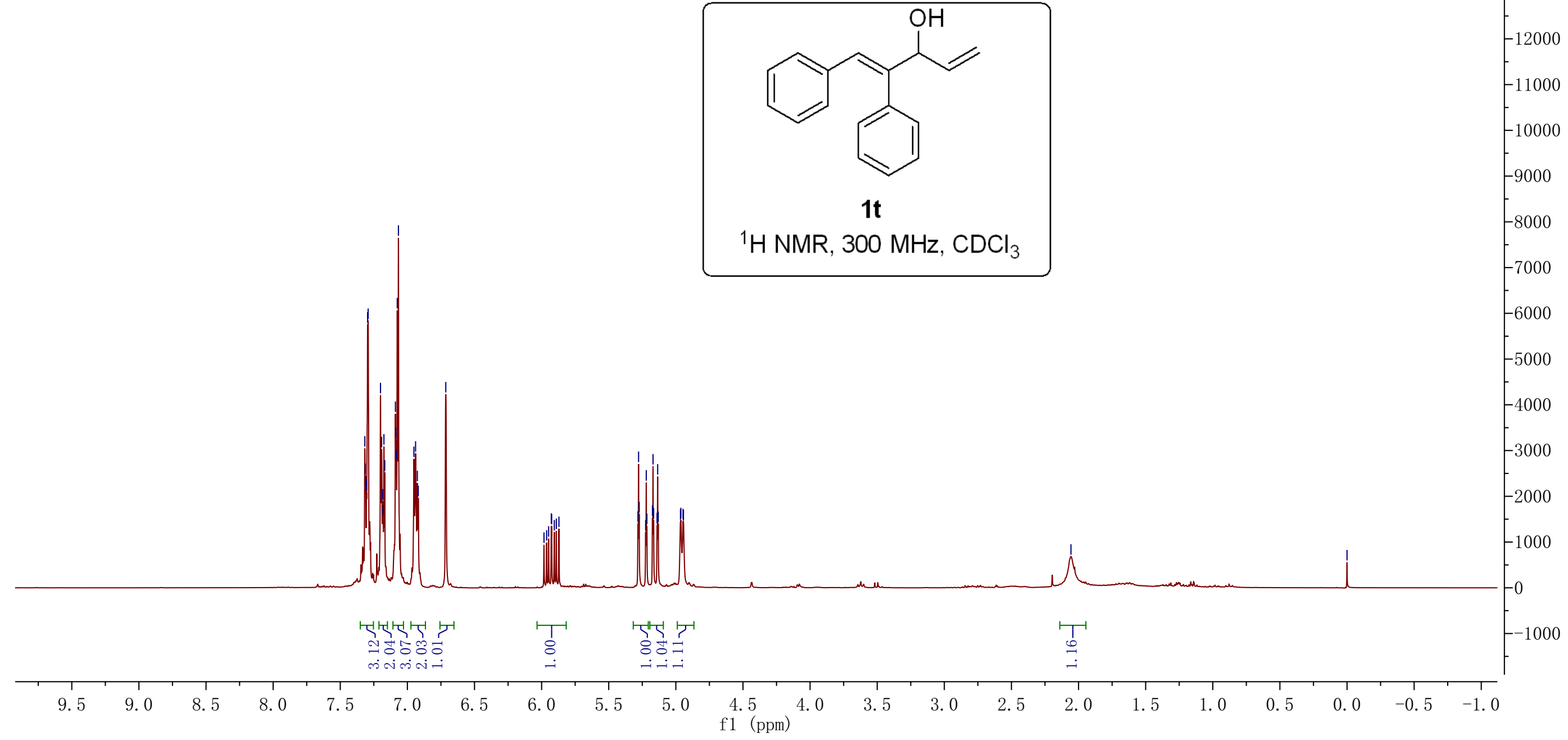

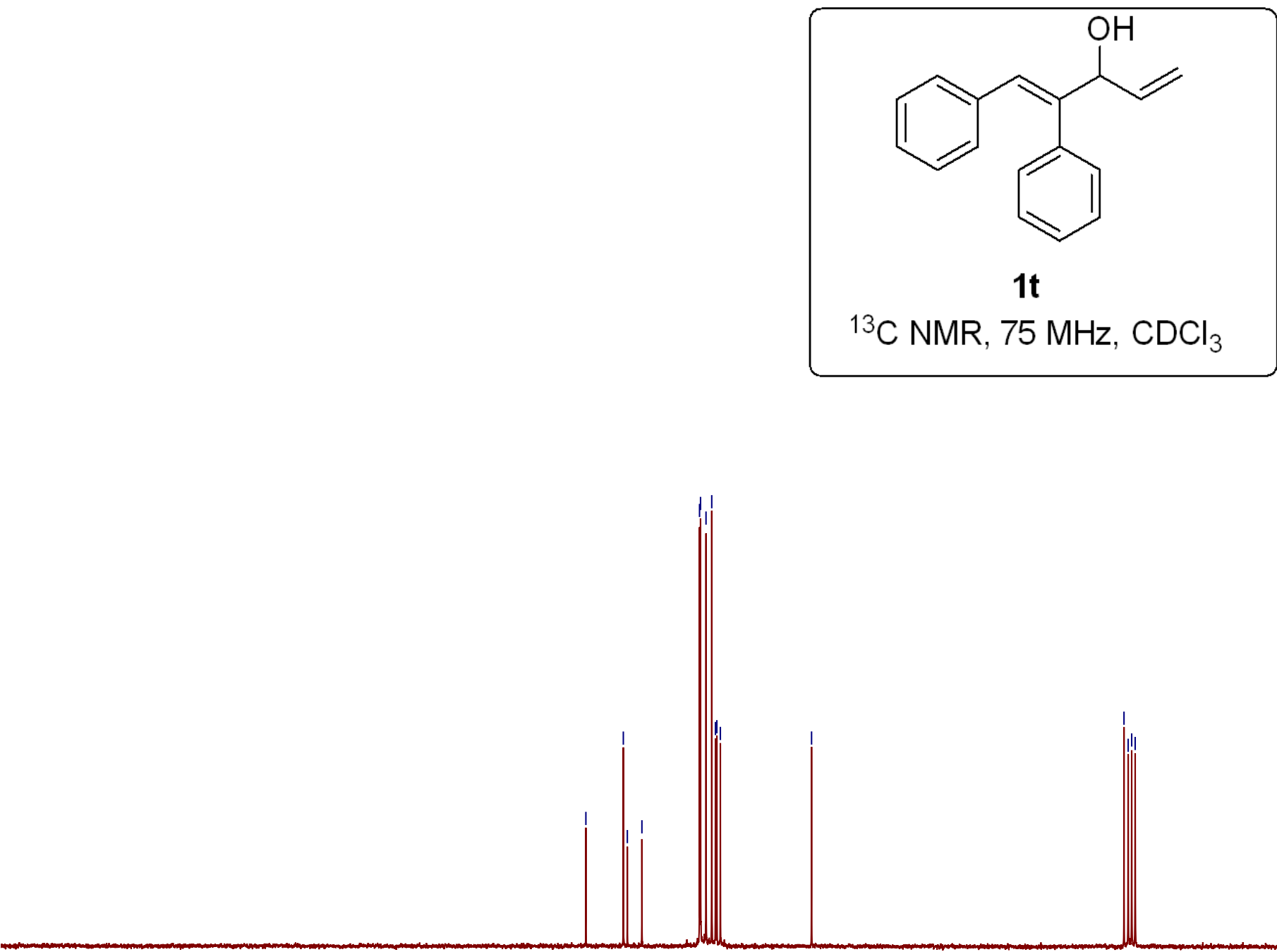

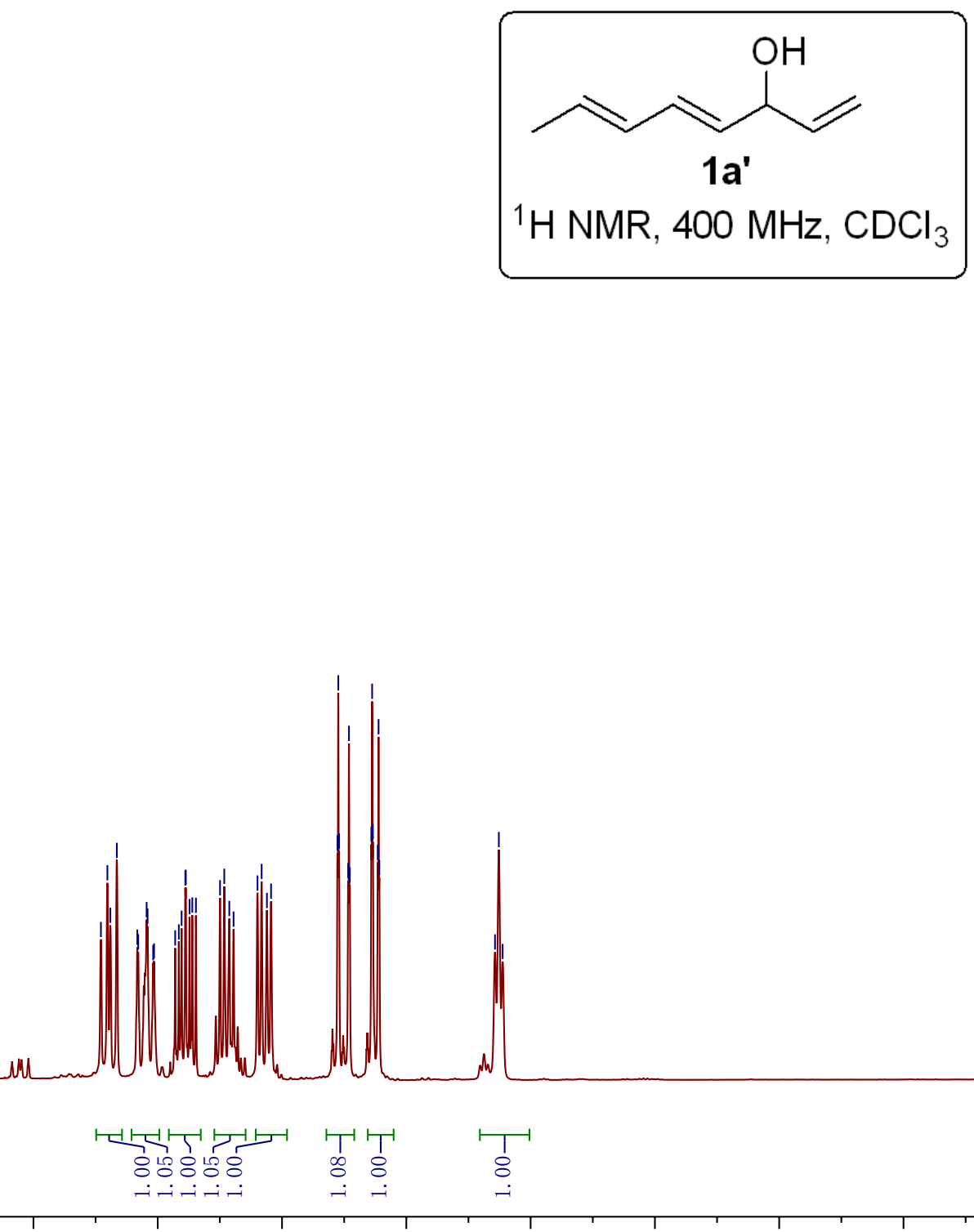

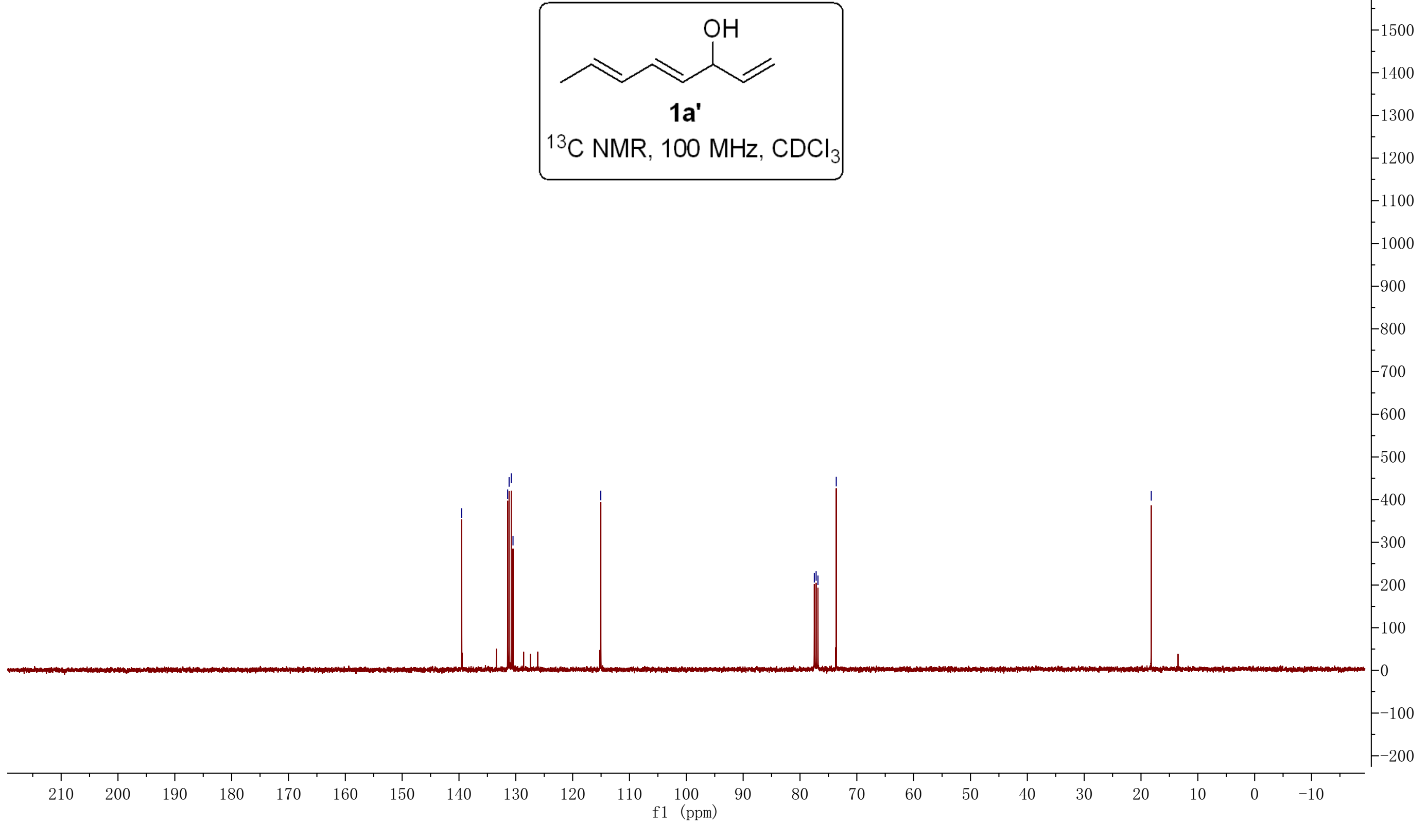

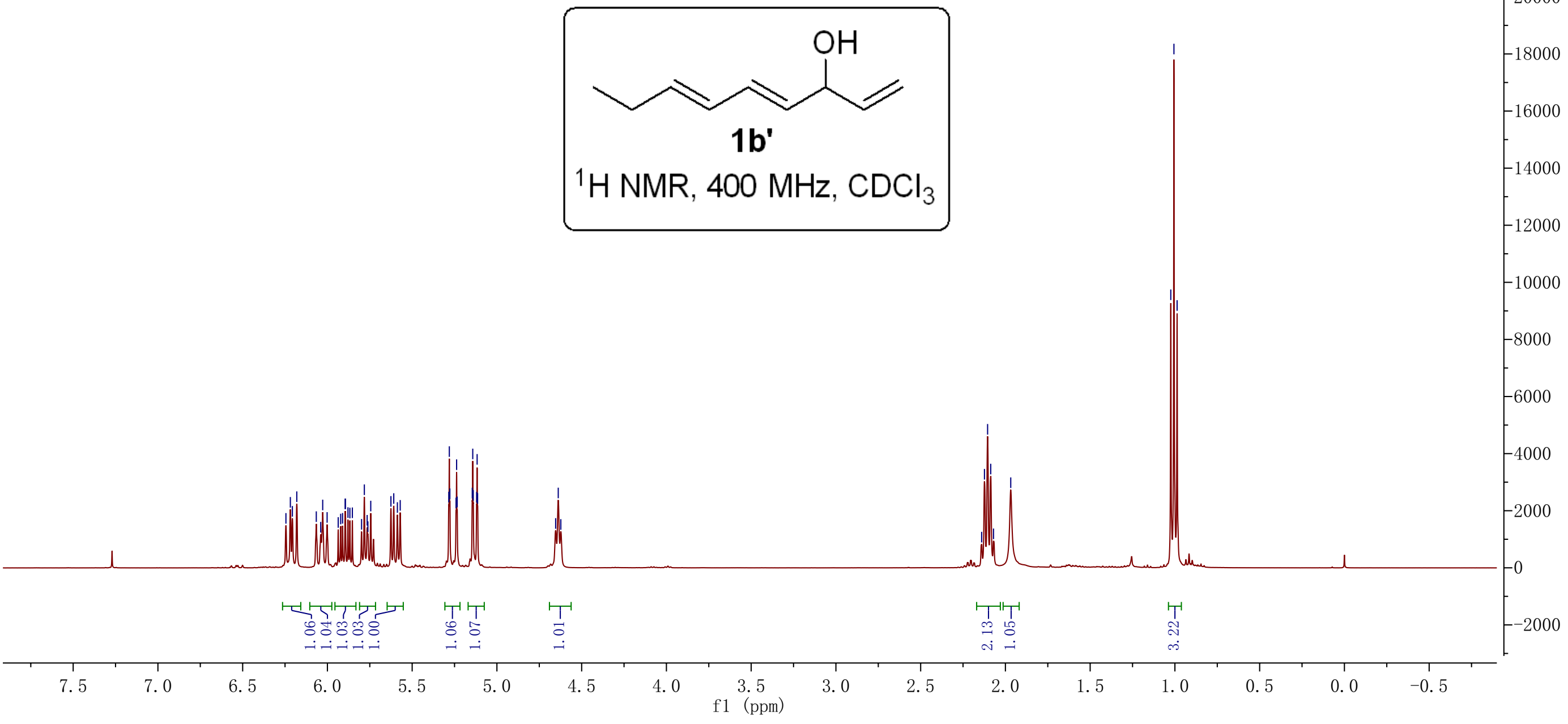


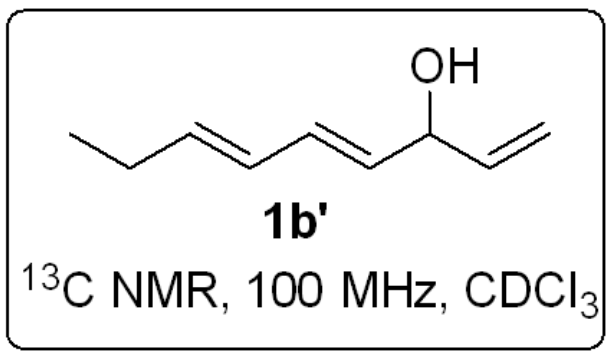




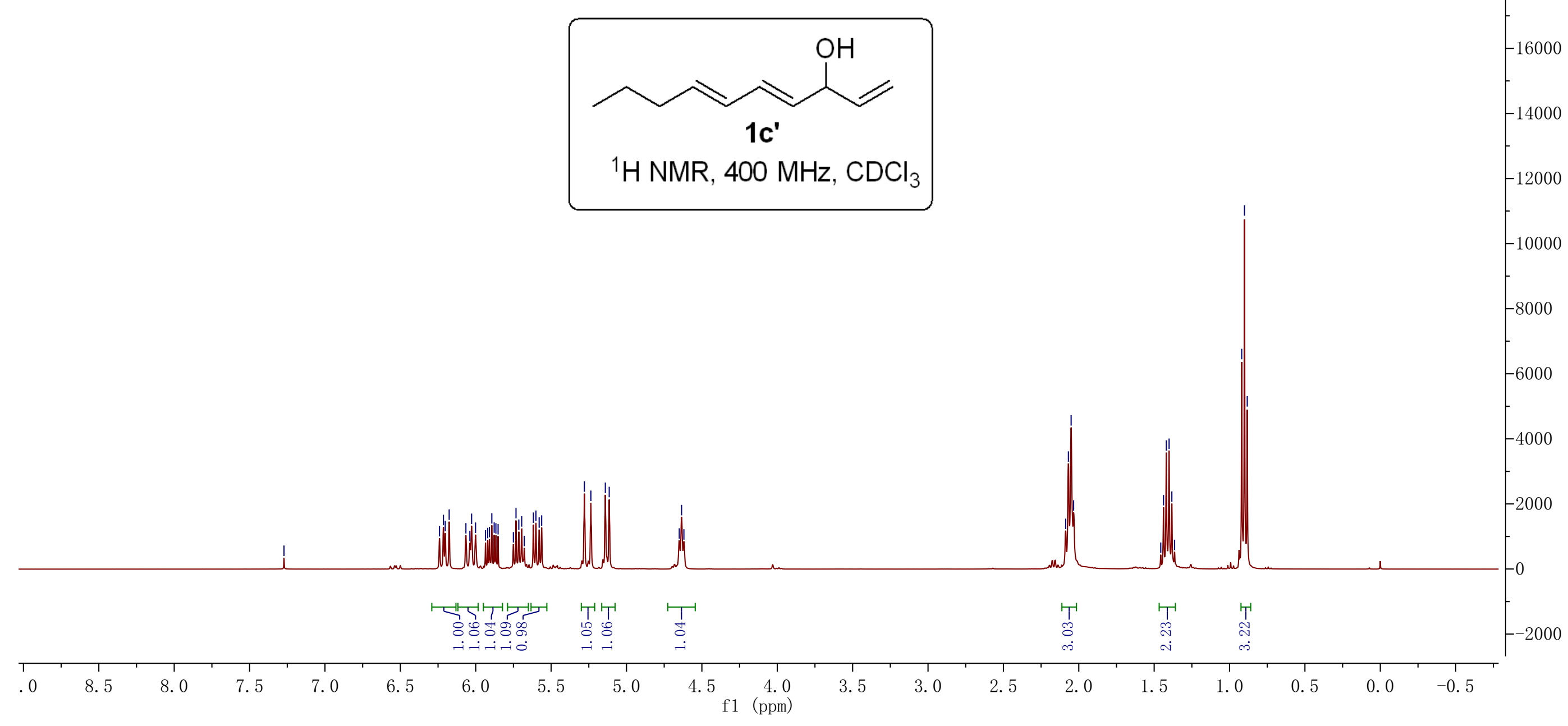



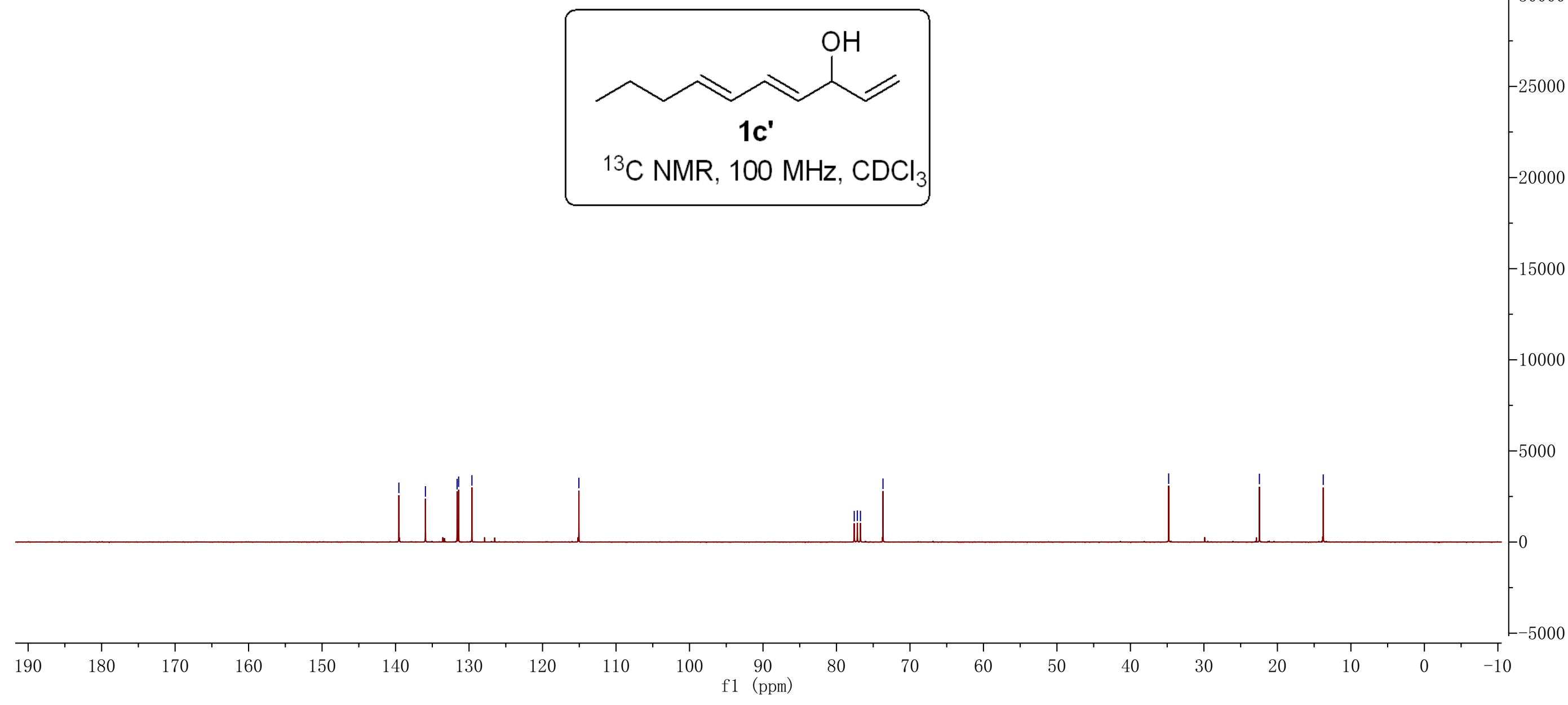


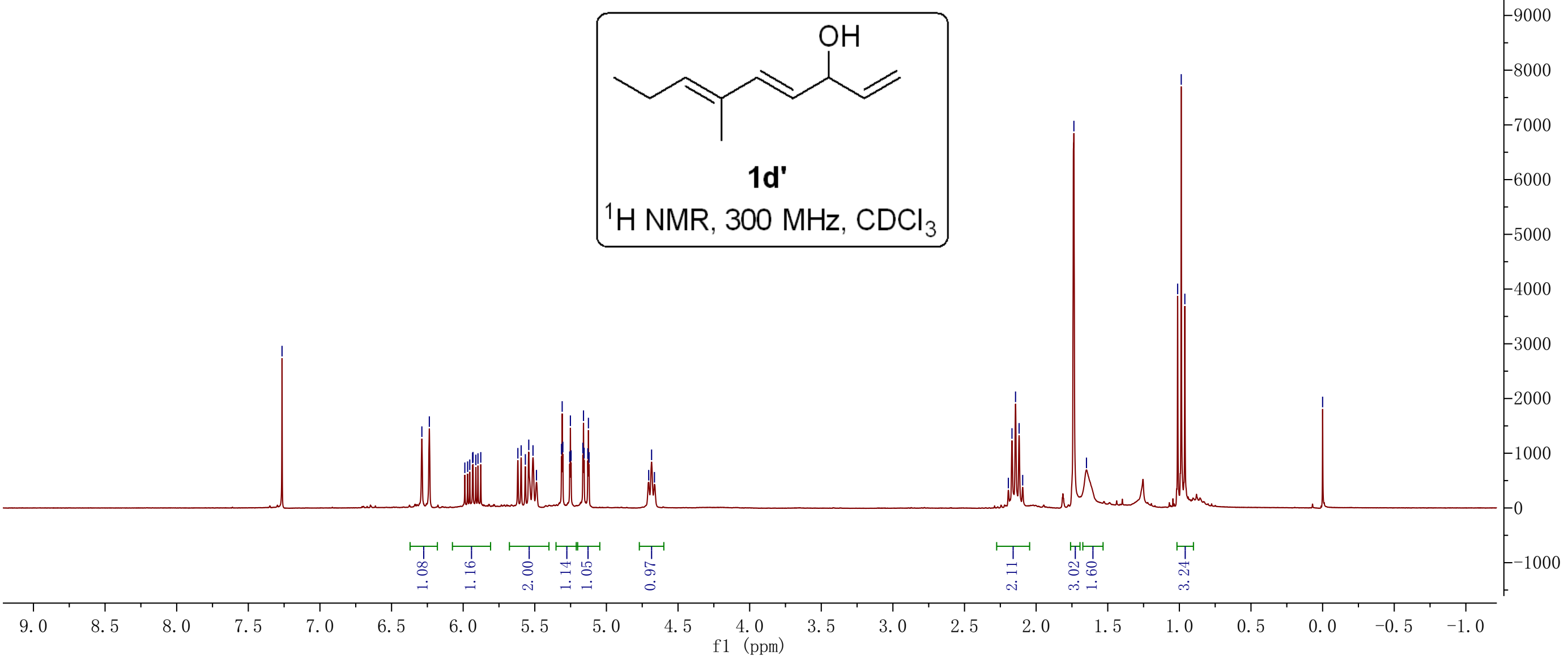




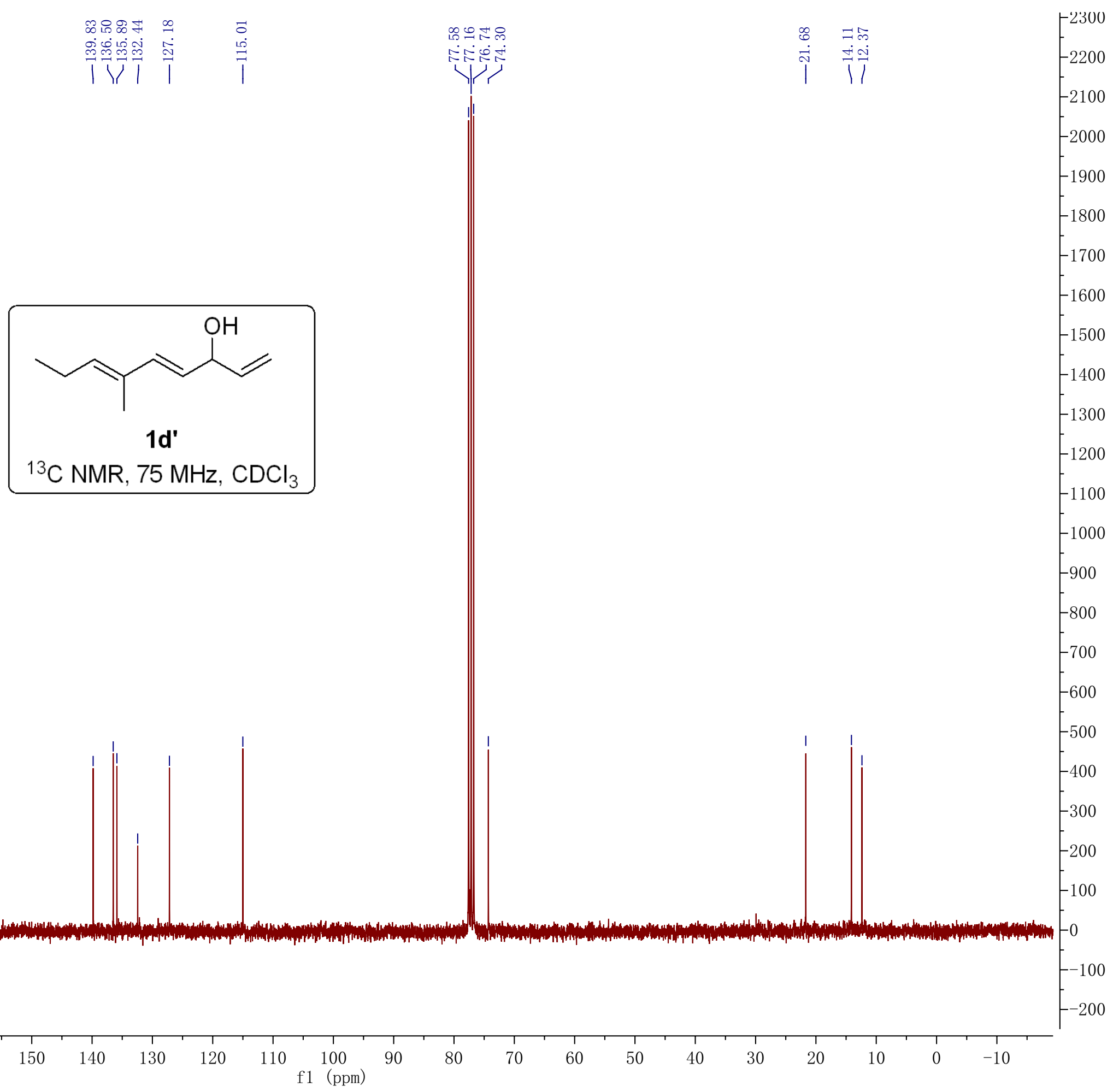



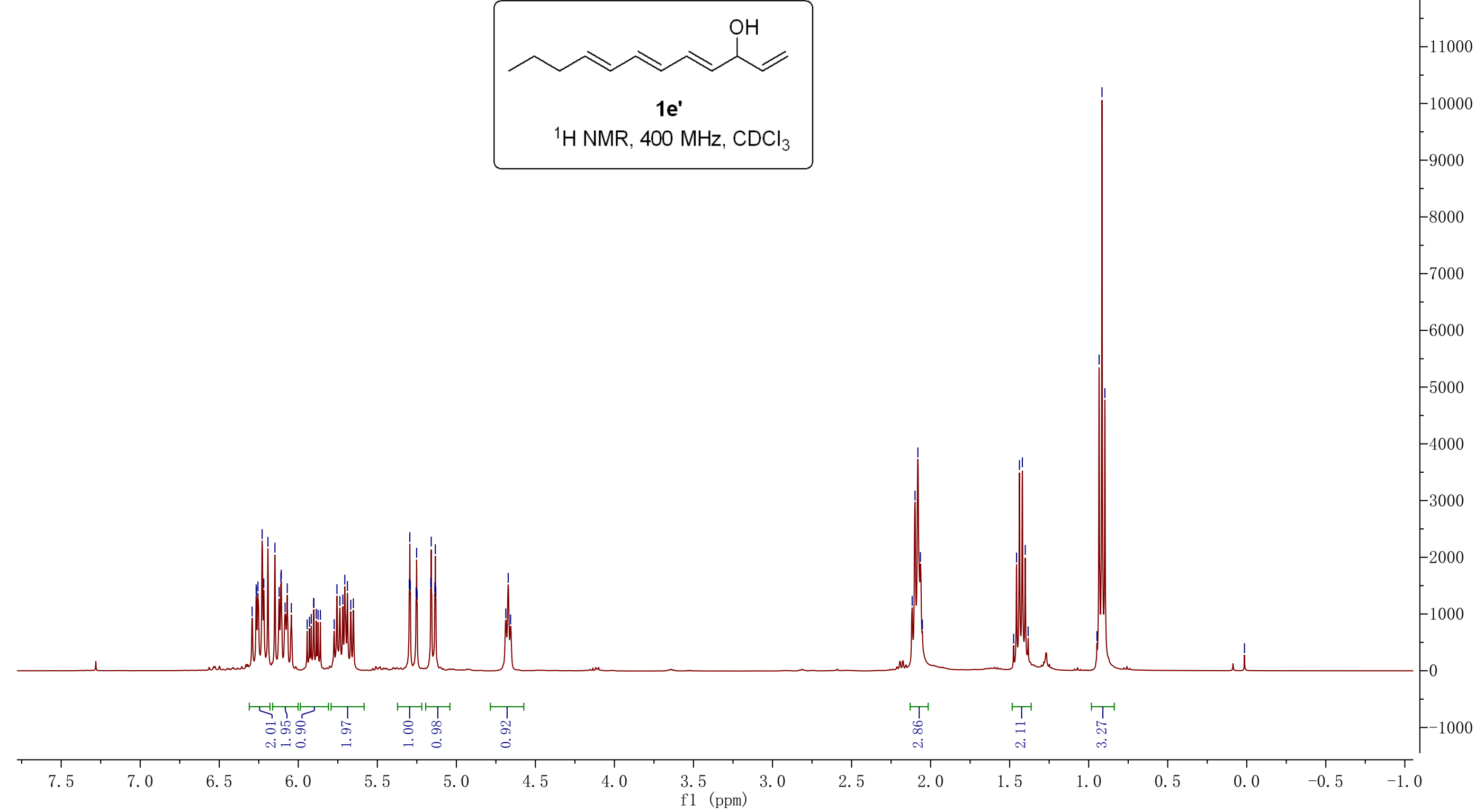


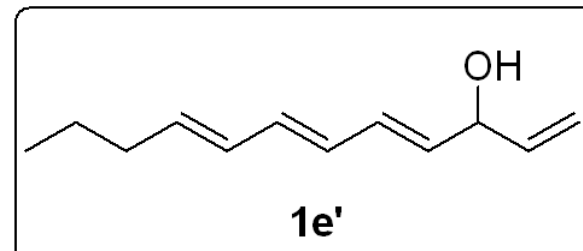

${ }^{13} \mathrm{C}$ NMR, $75 \mathrm{MHz}, \mathrm{CDCl}_{3}$

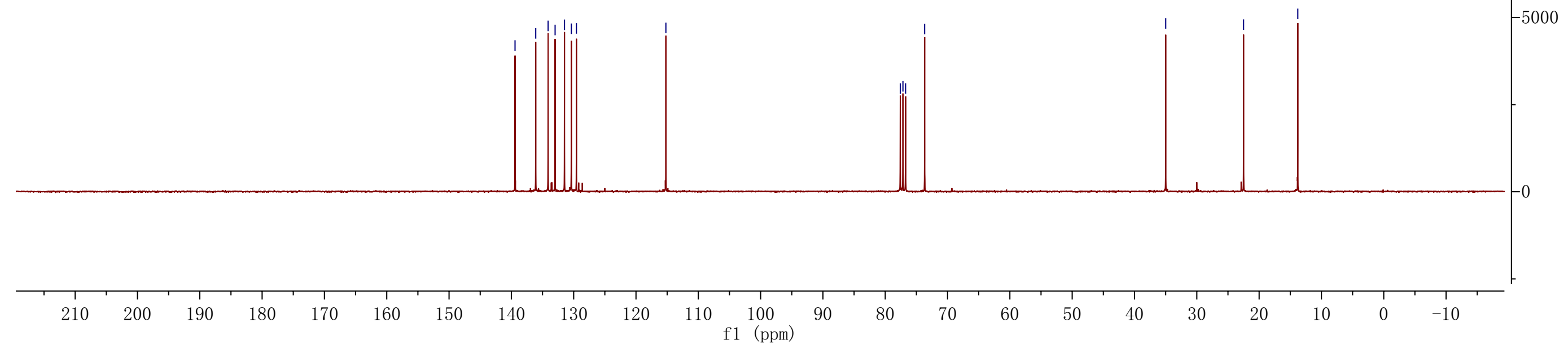




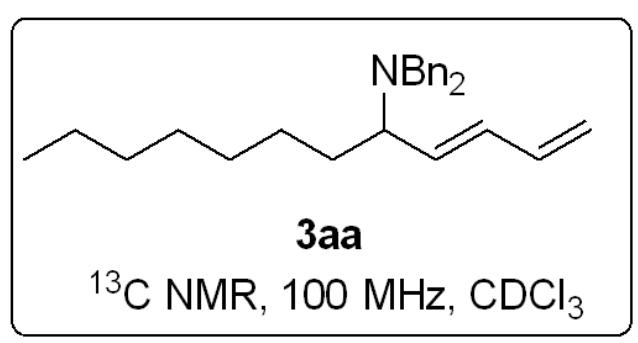



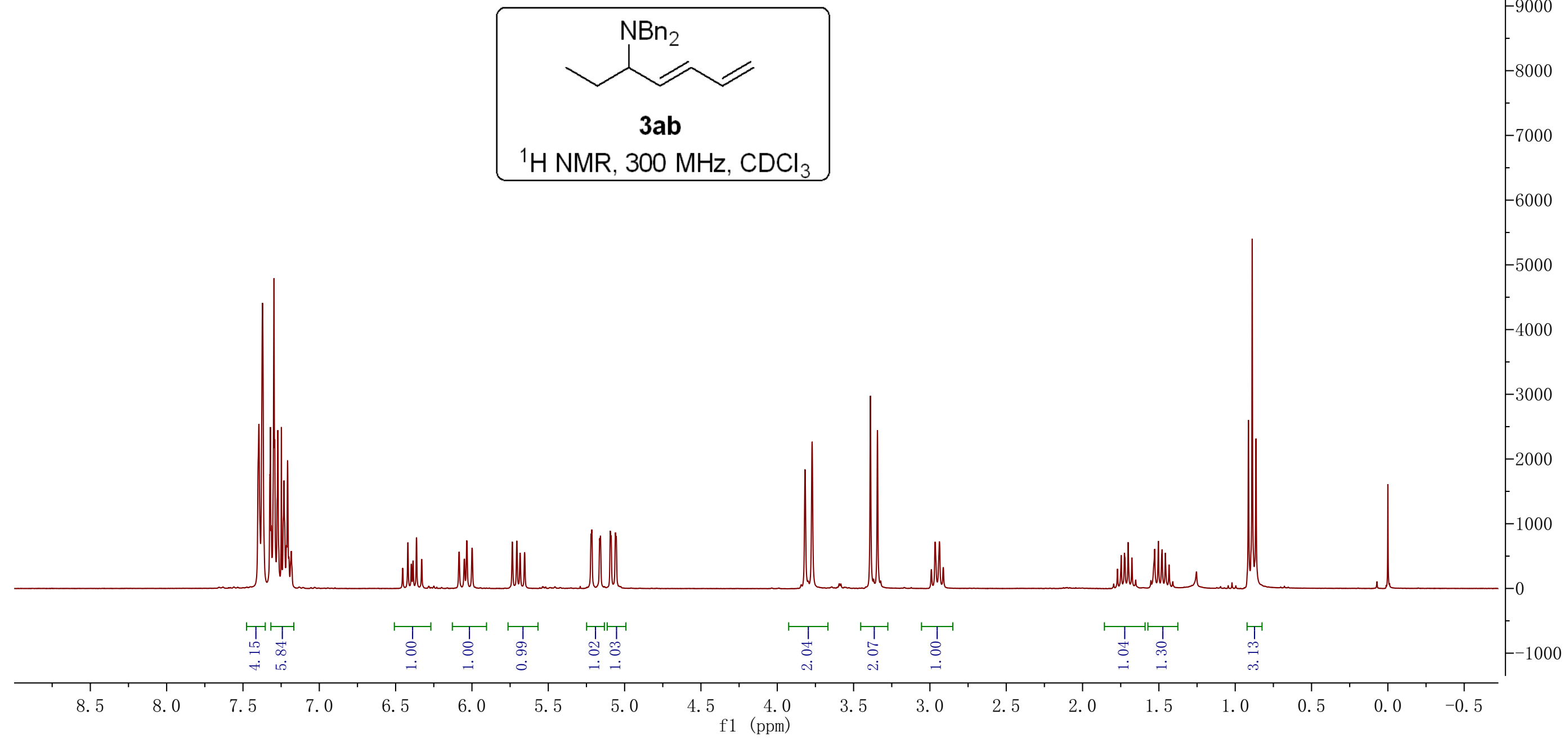


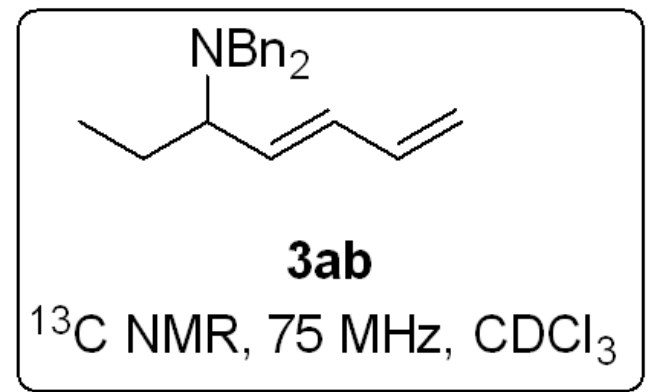



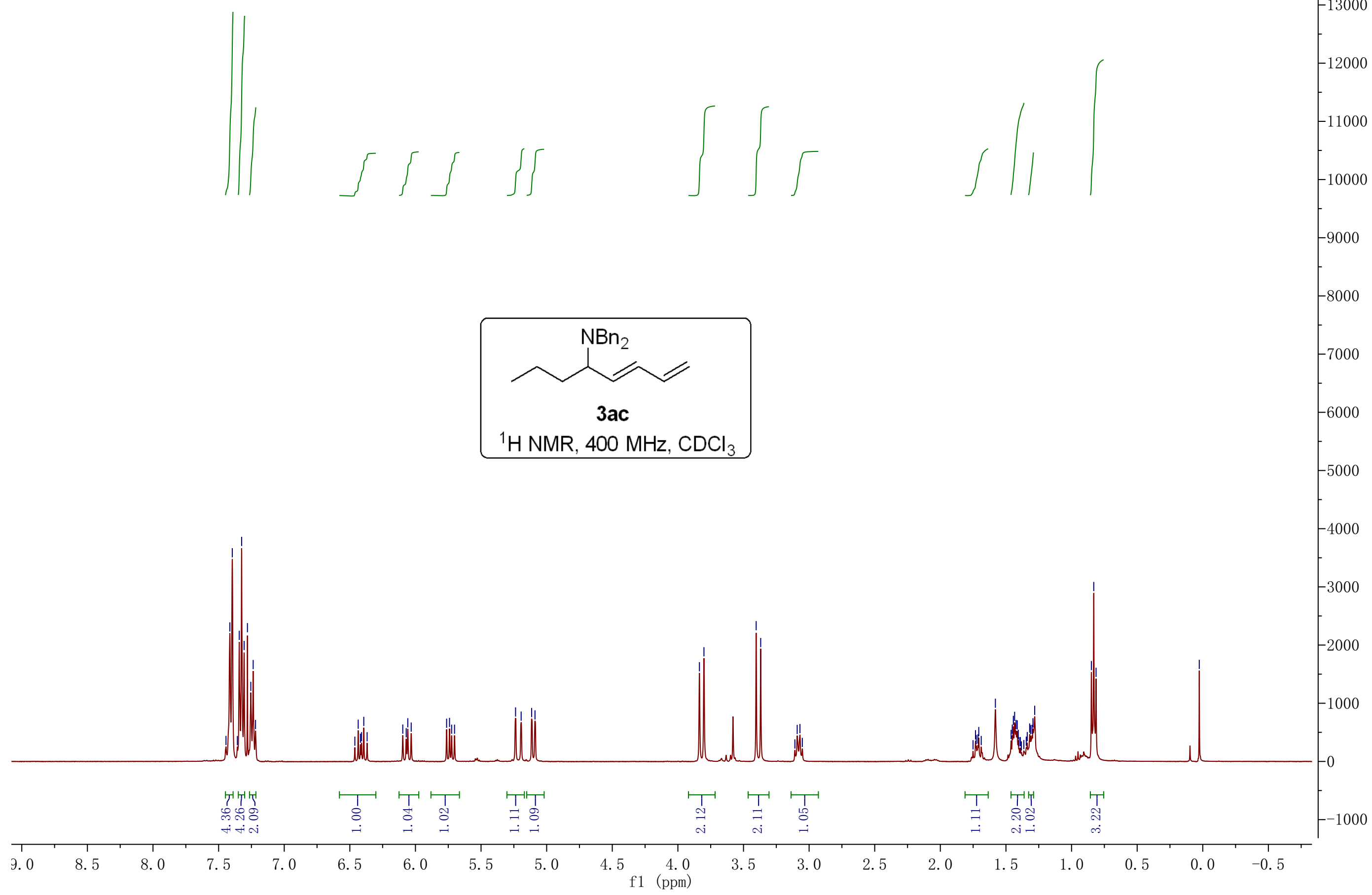

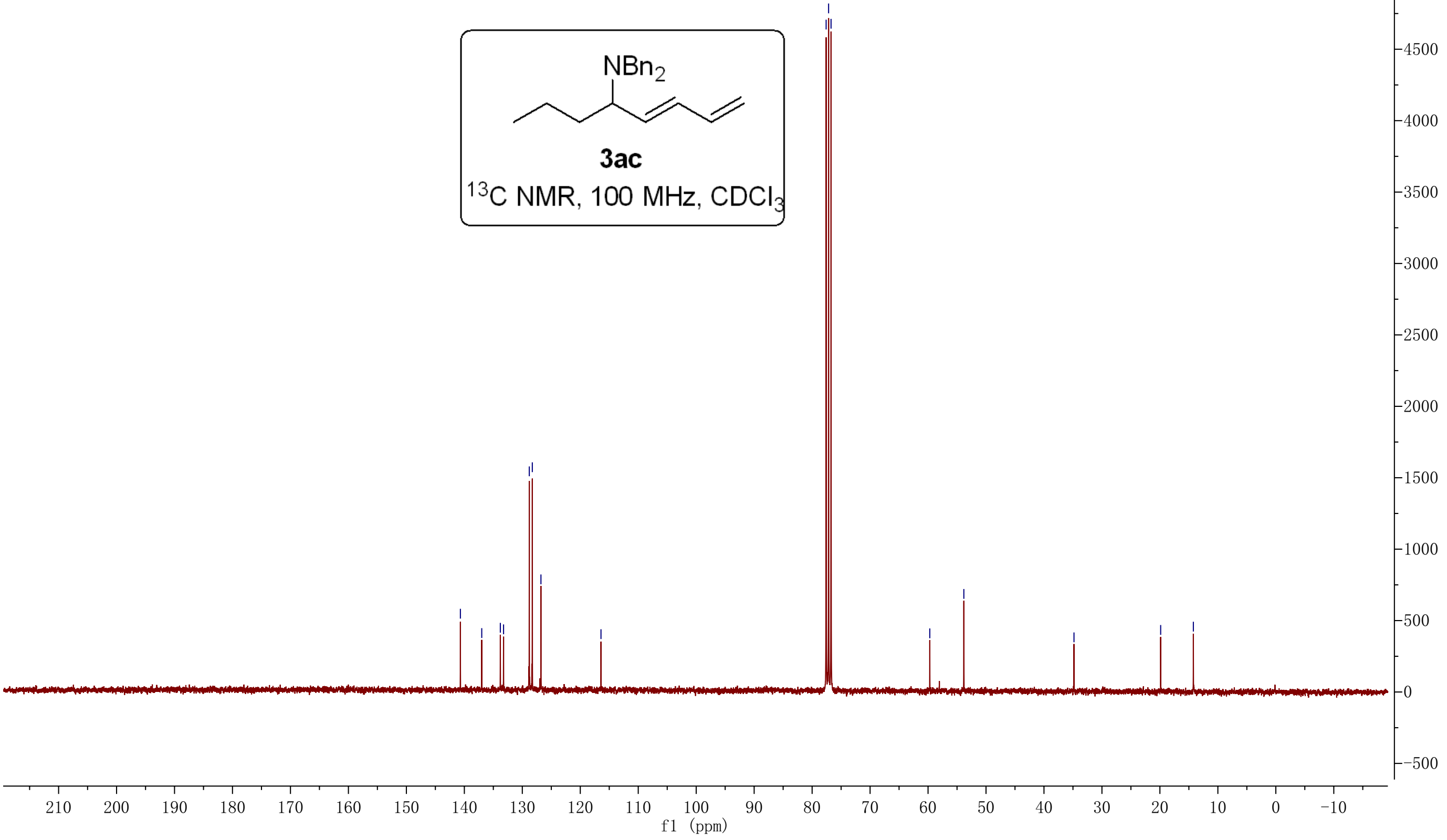

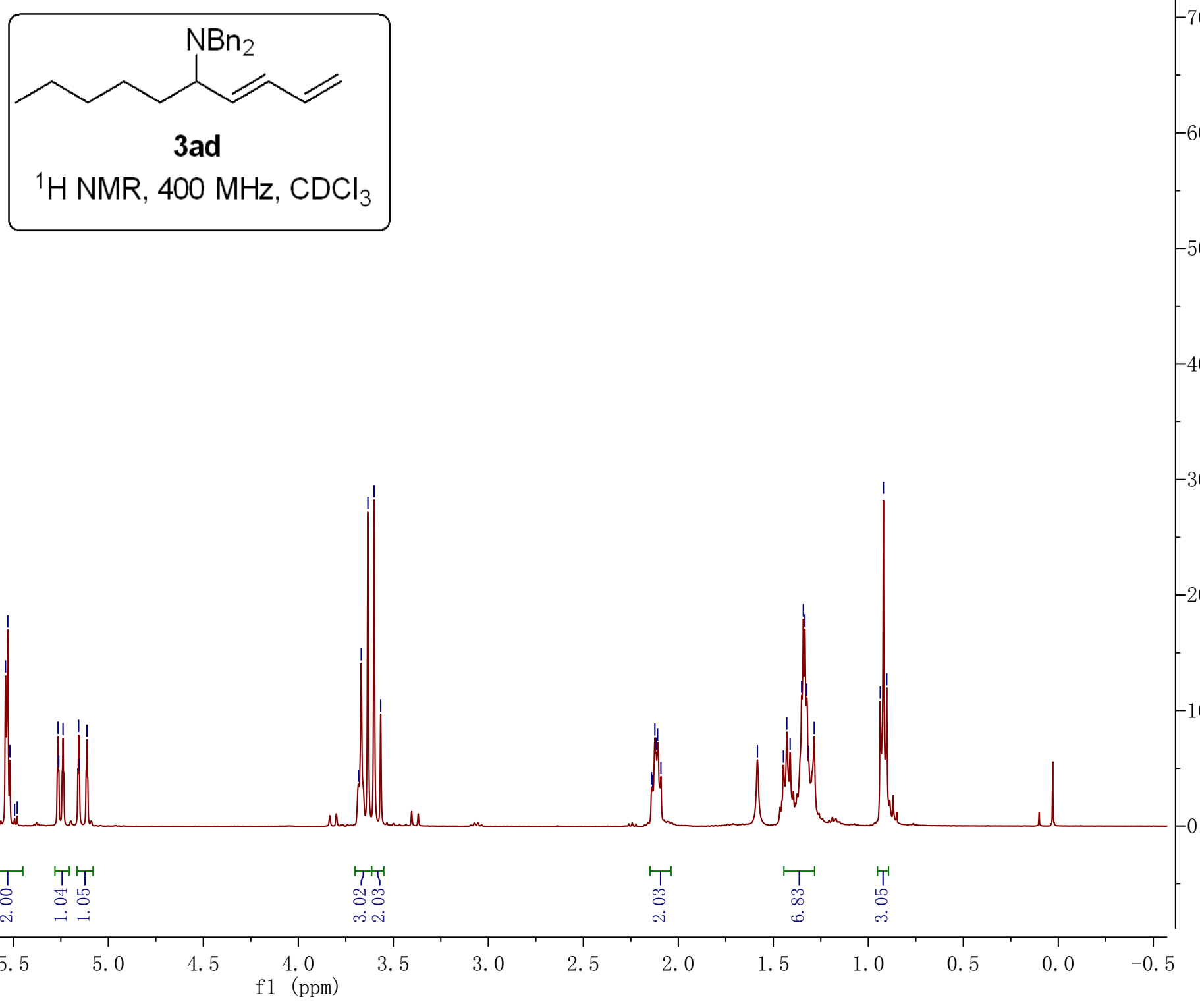

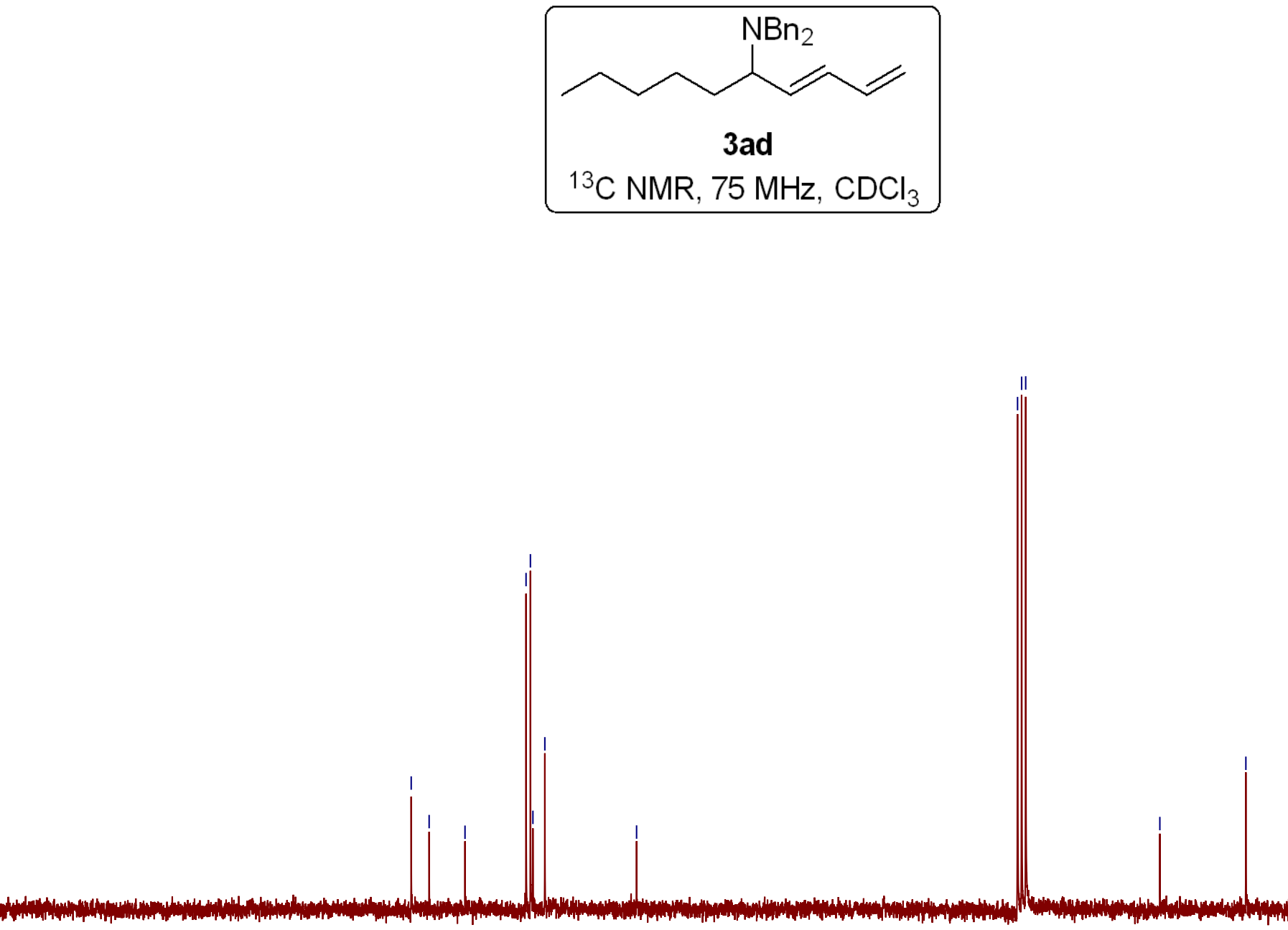

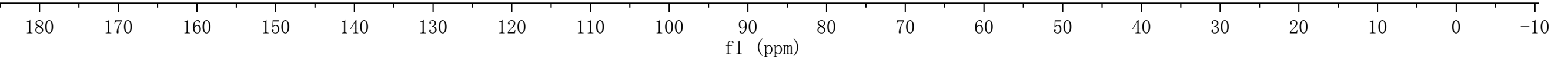



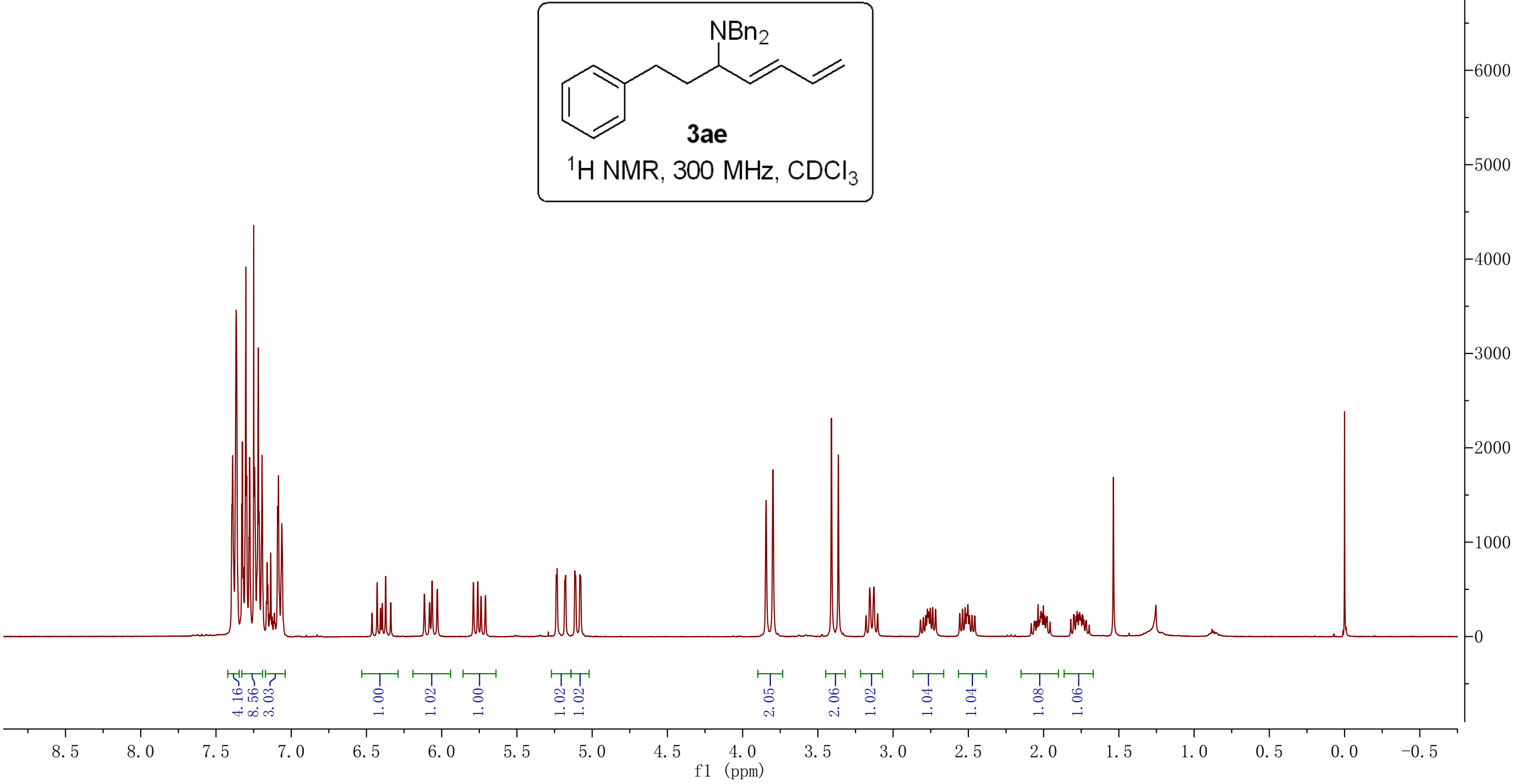

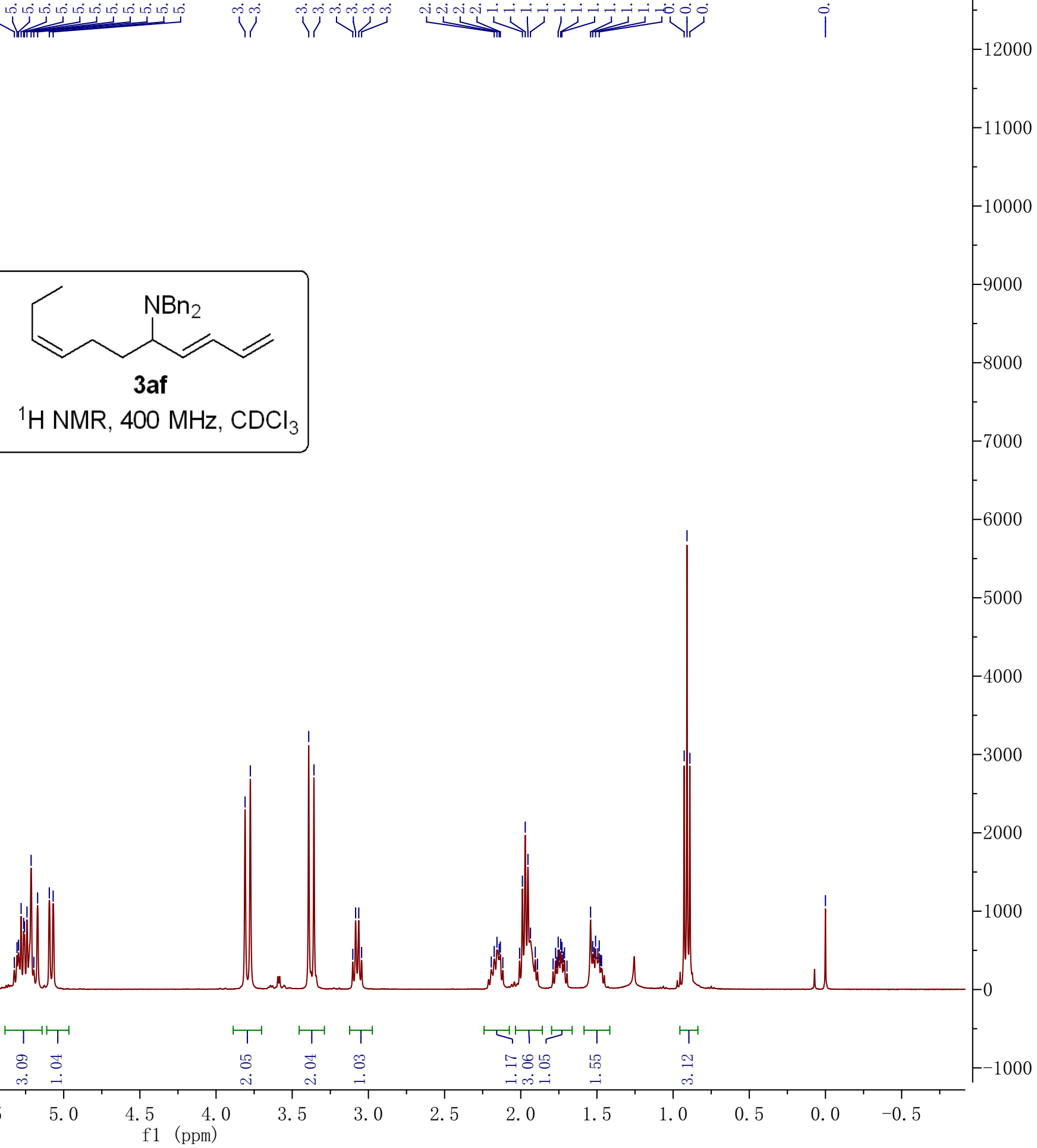


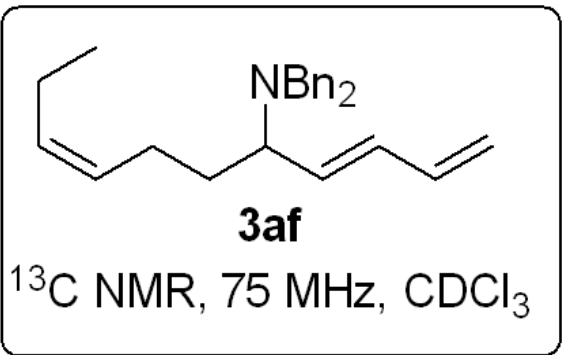




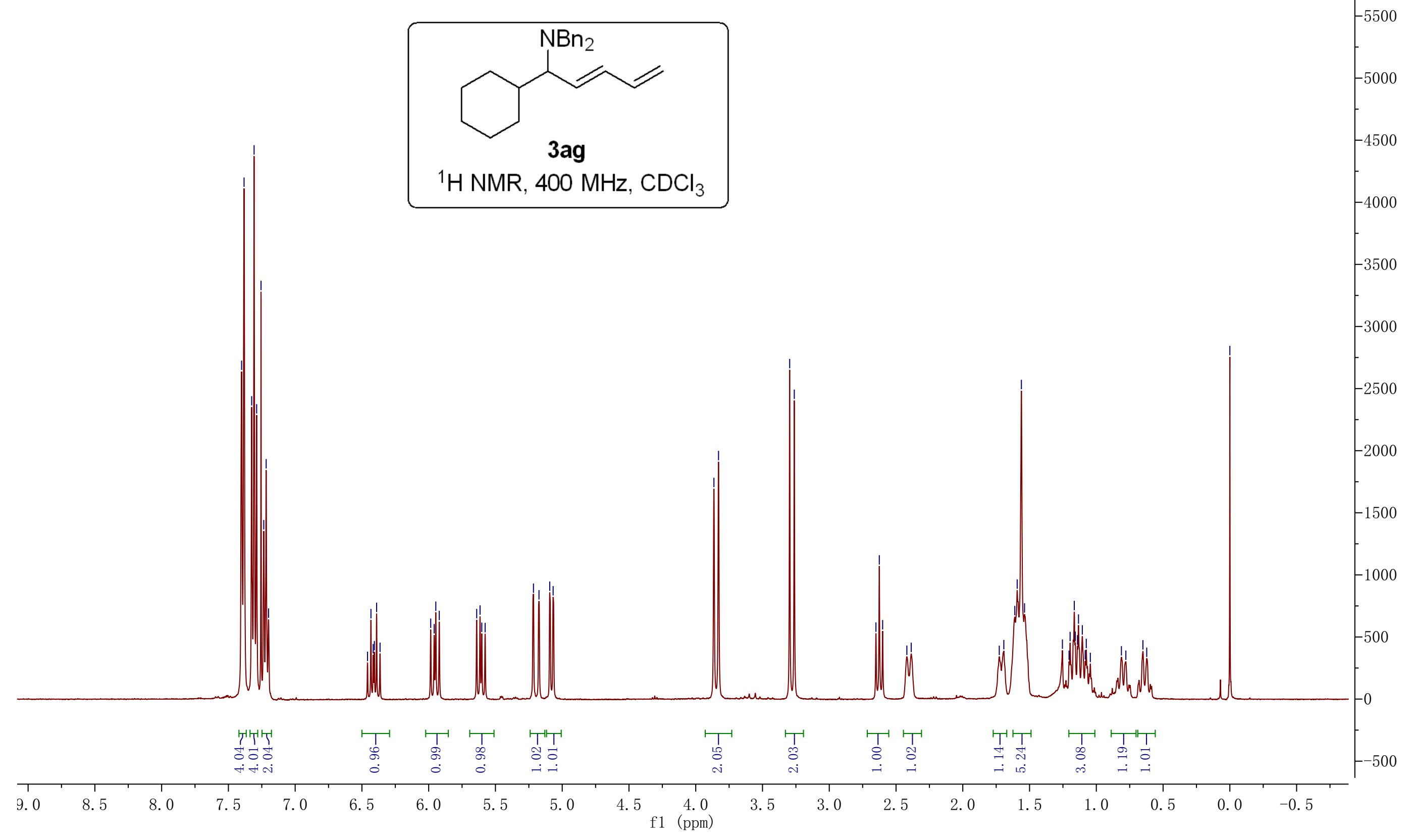



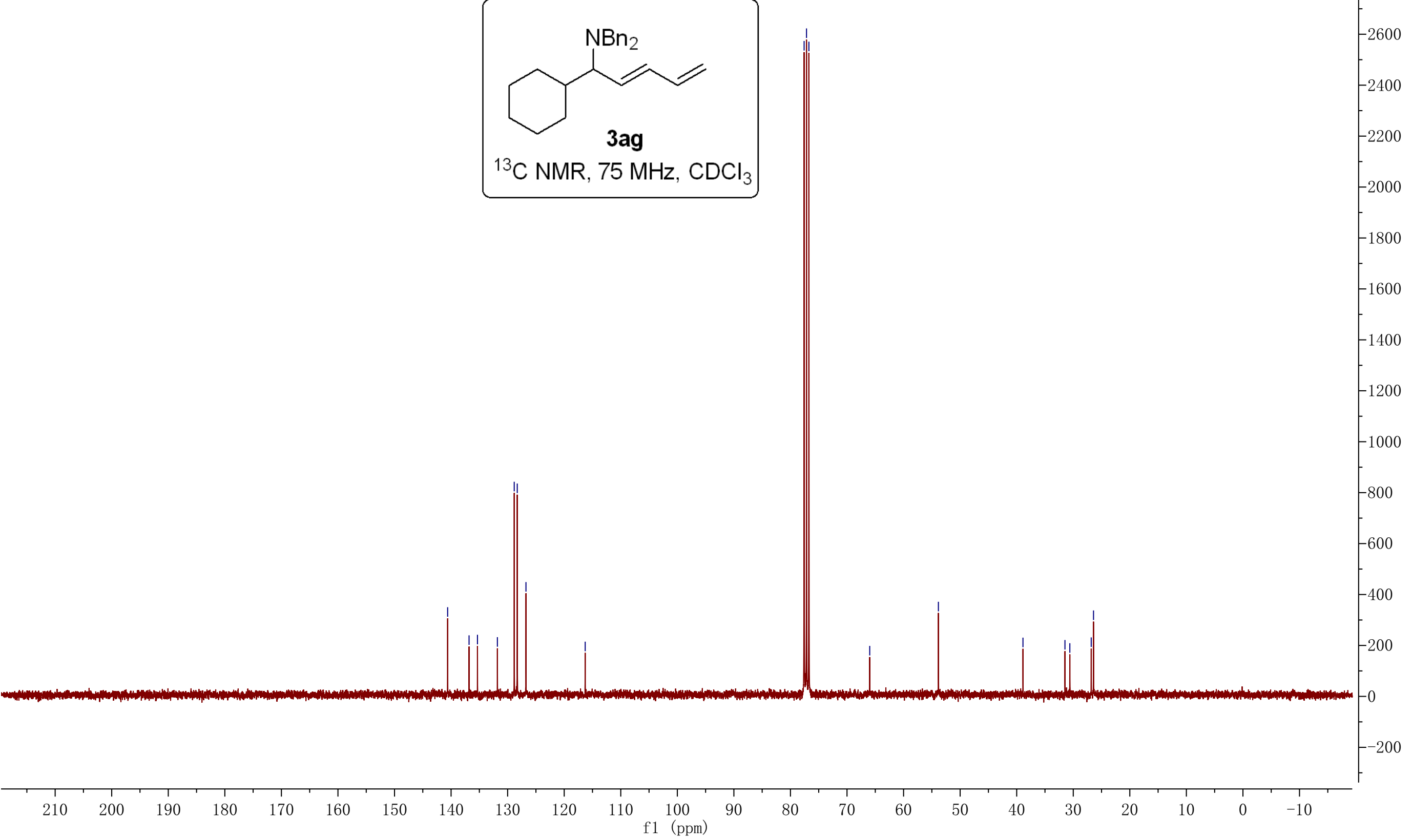


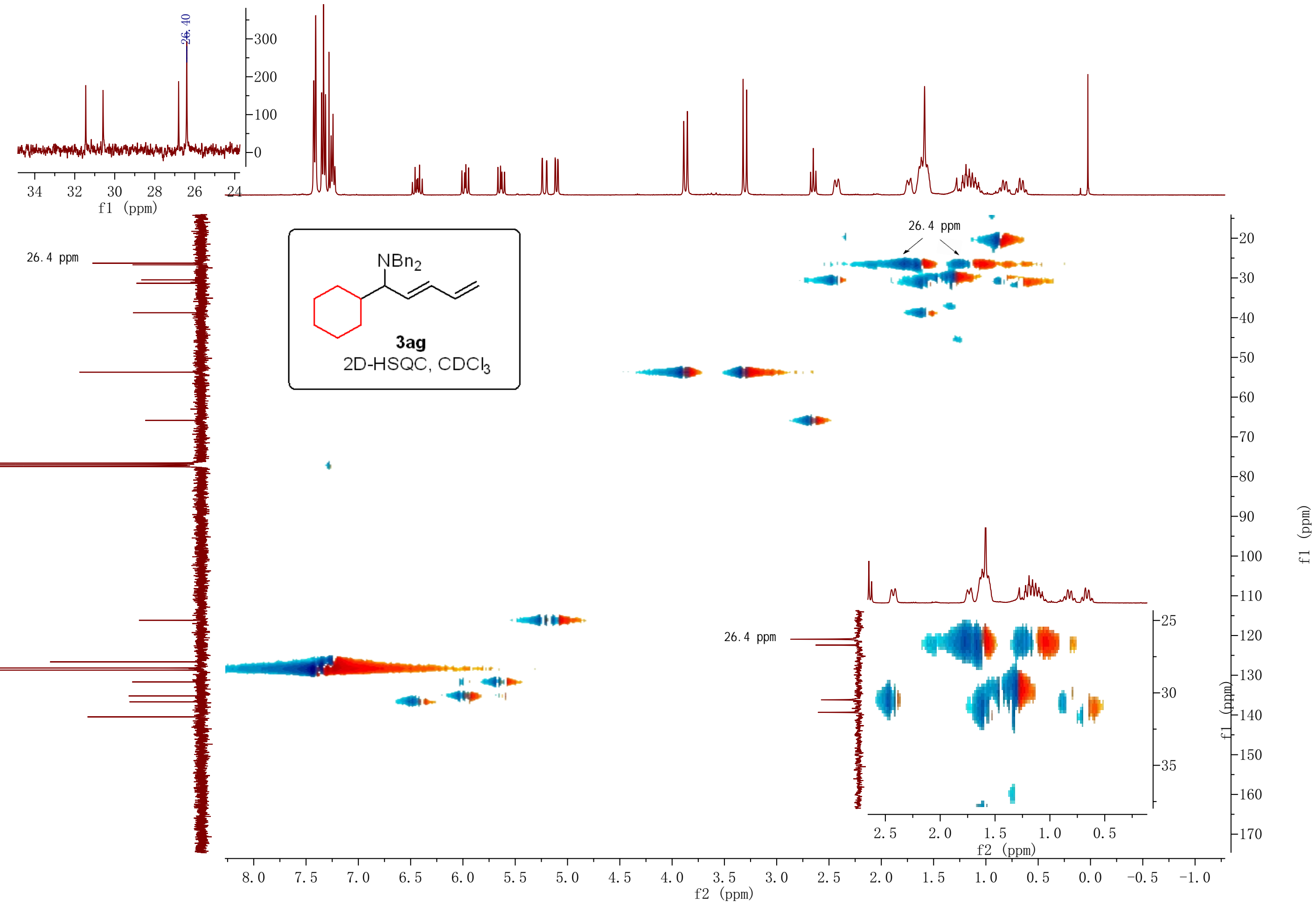




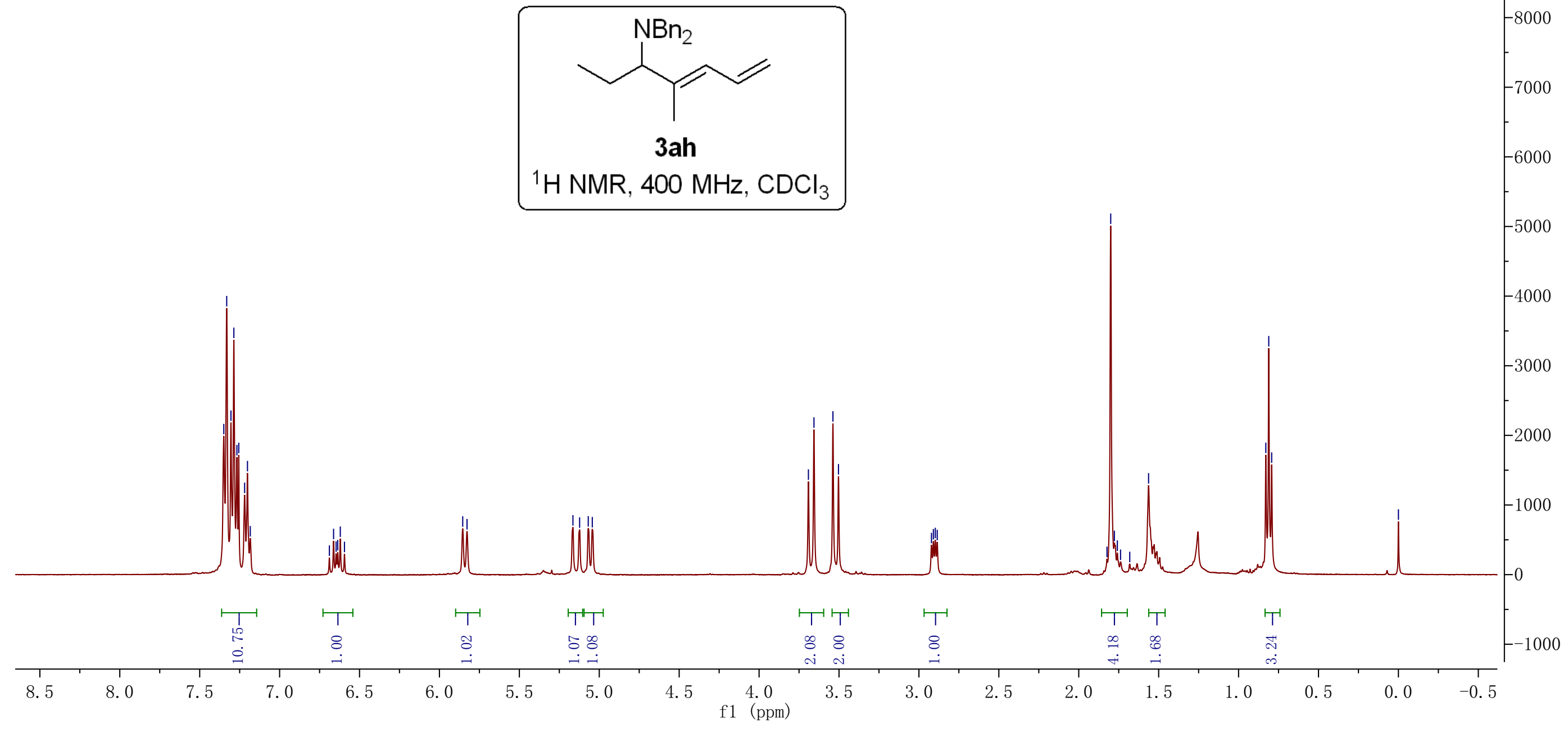



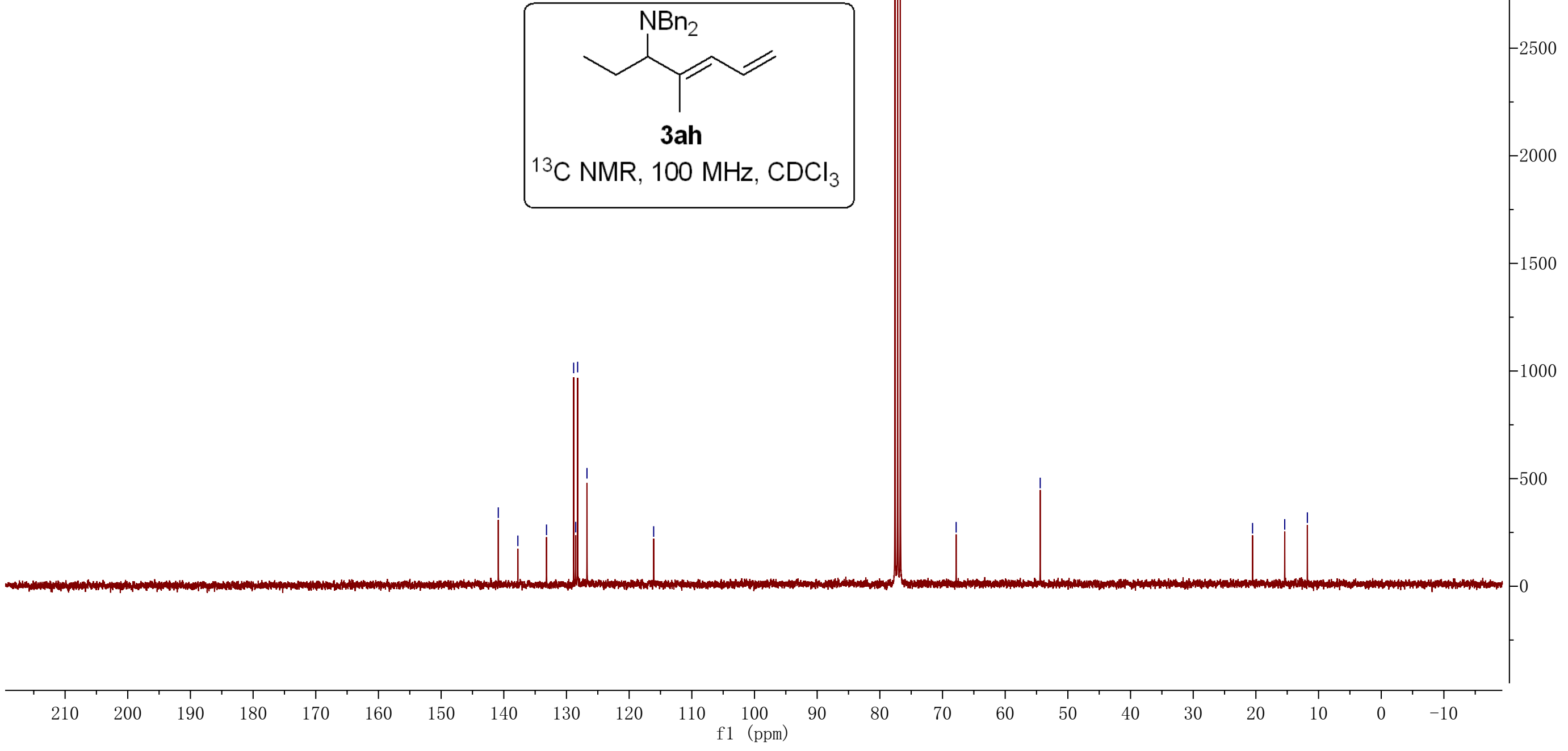

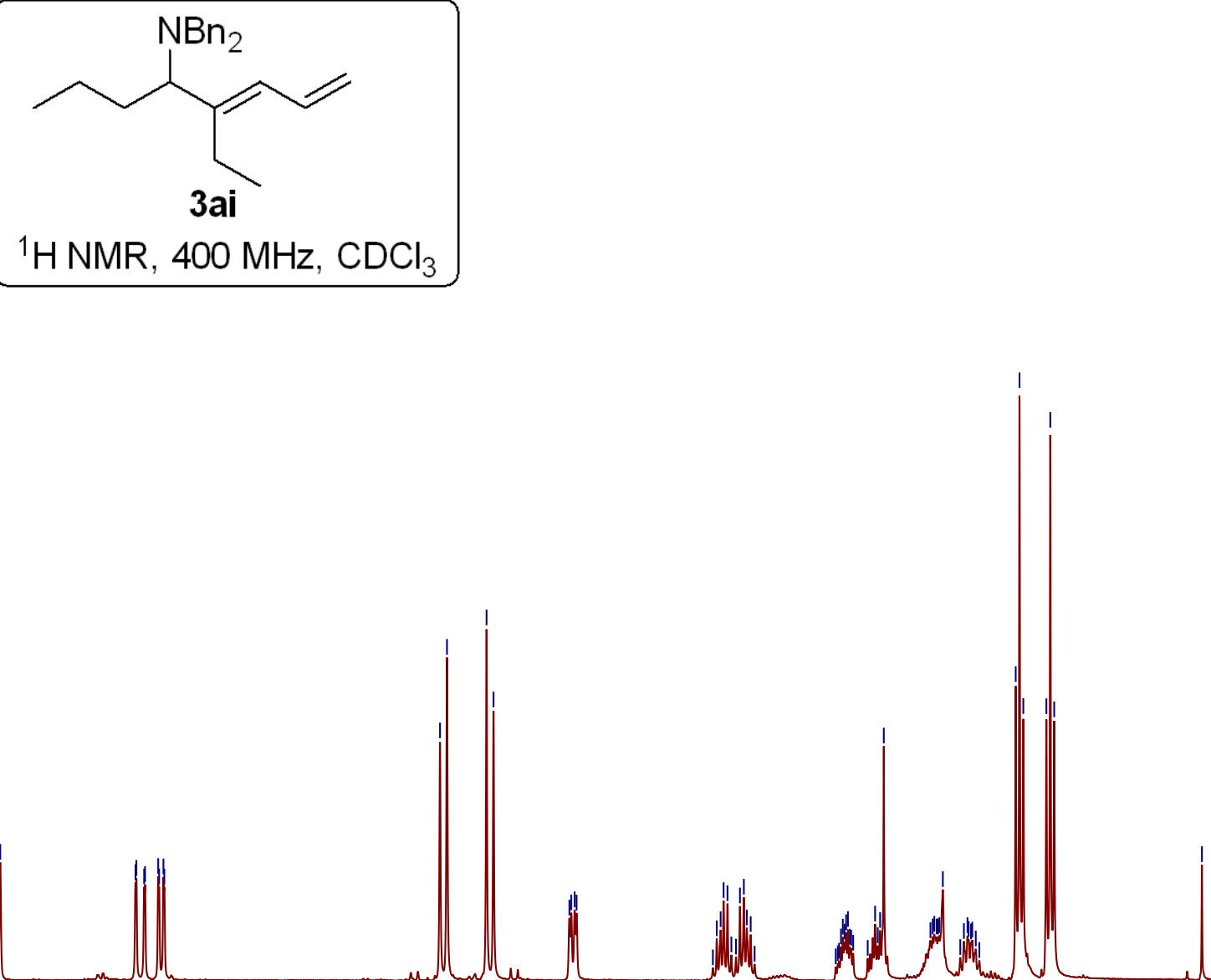

$1.5 \quad 1.0 \quad 0.5$




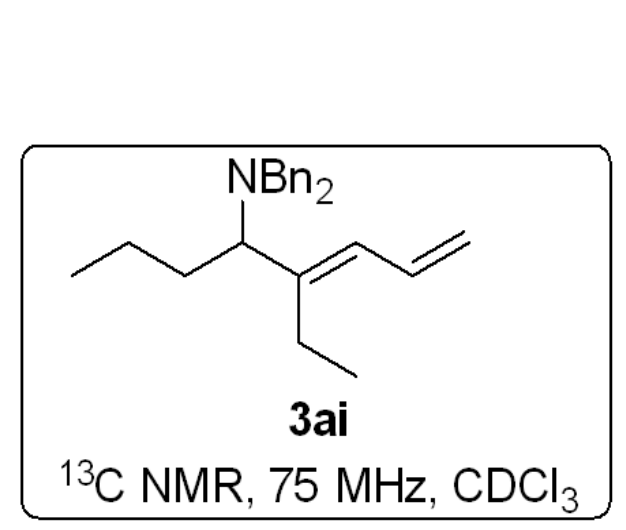




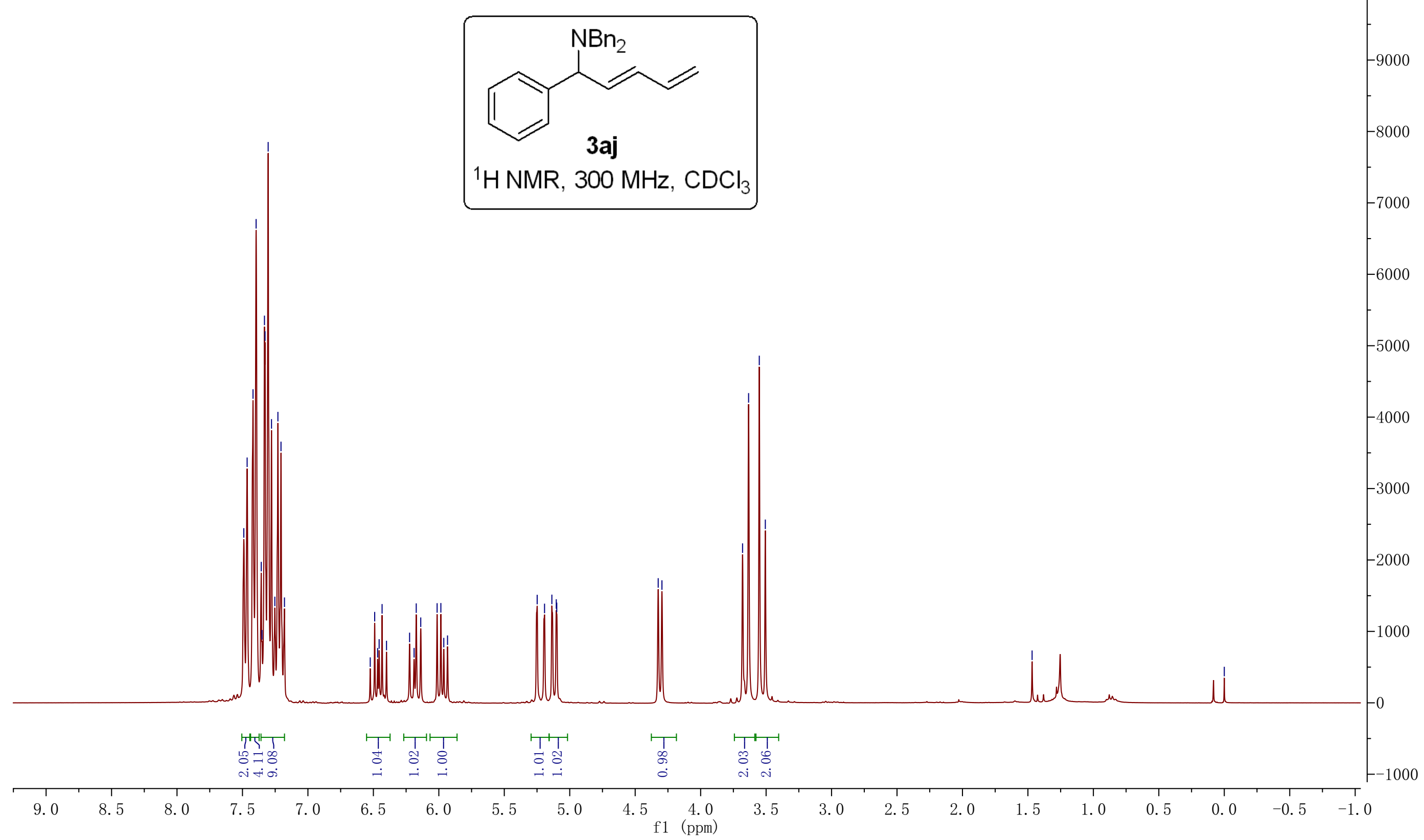




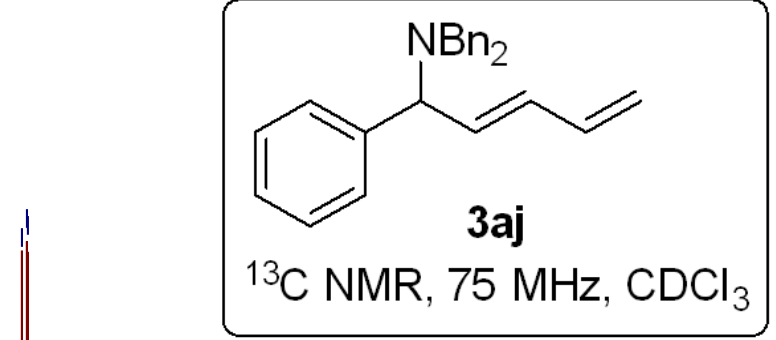



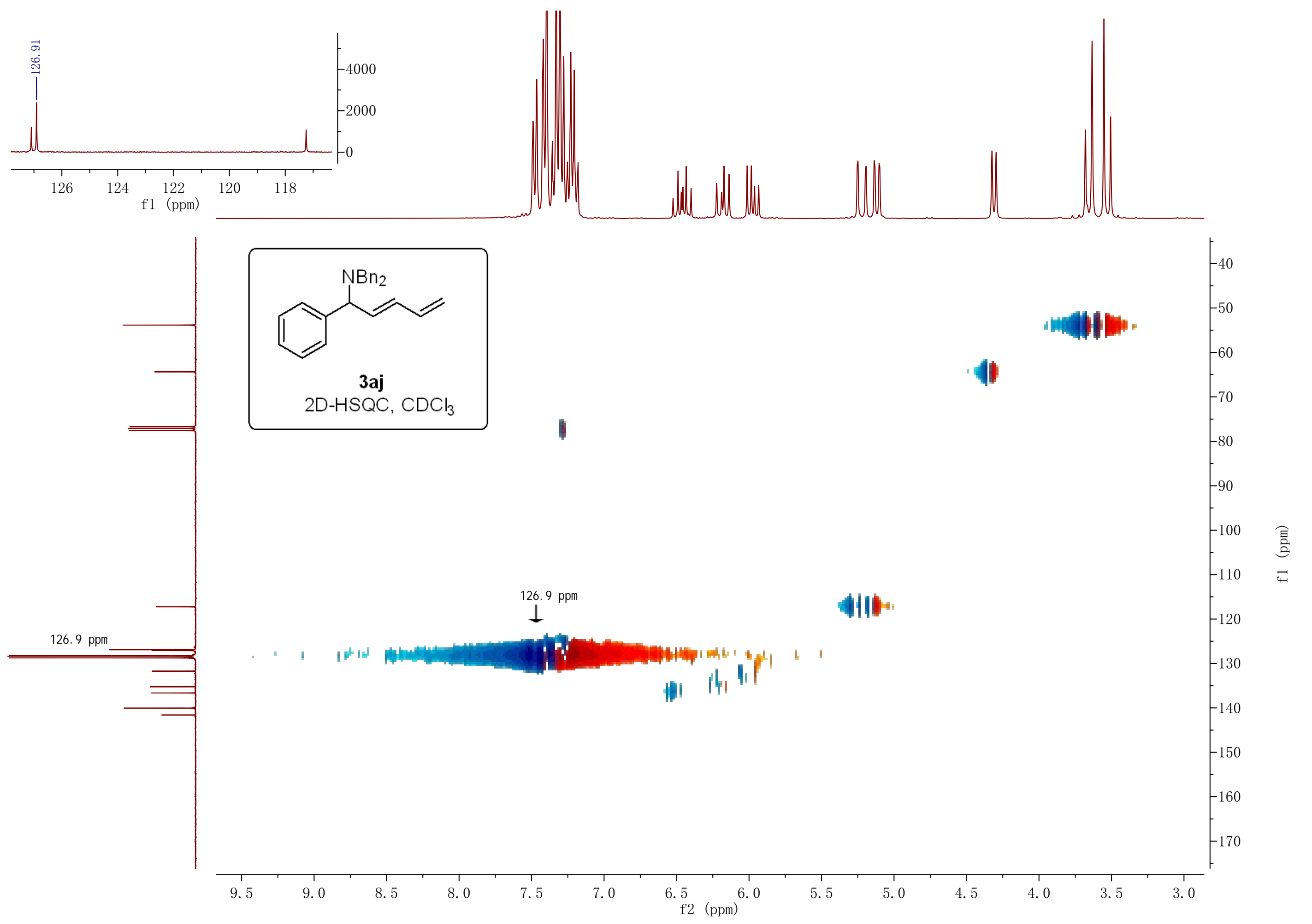


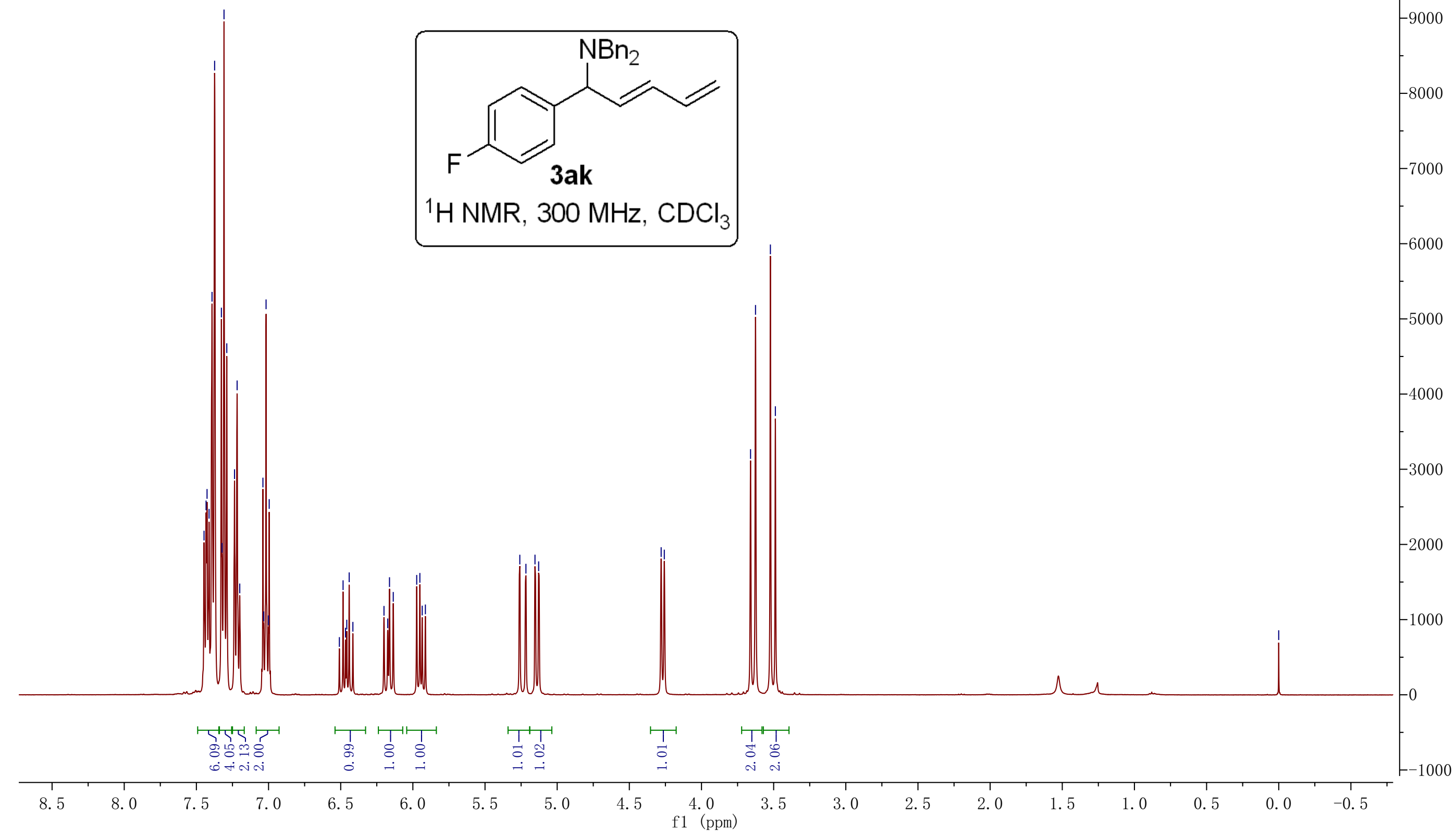




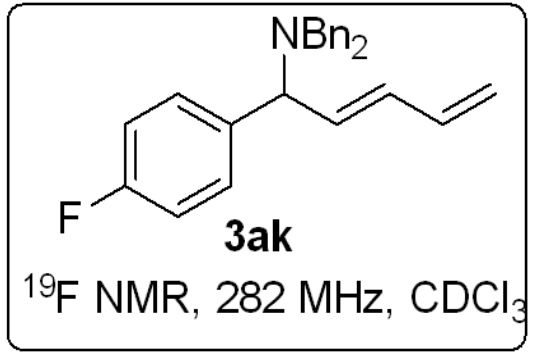




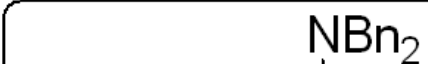

${ }^{1} \mathrm{HNMR}, 400 \mathrm{MHz}, \mathrm{CDCl}_{3}$
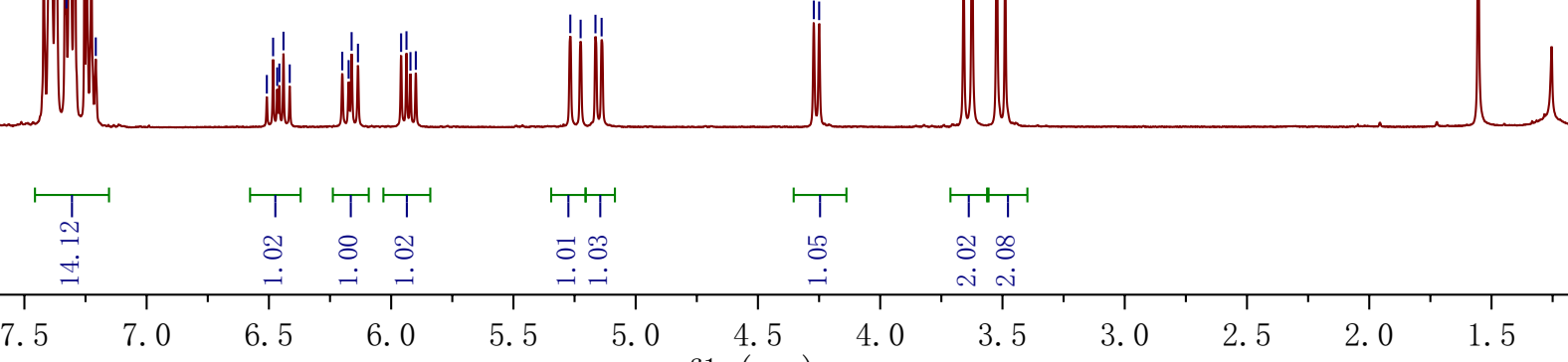


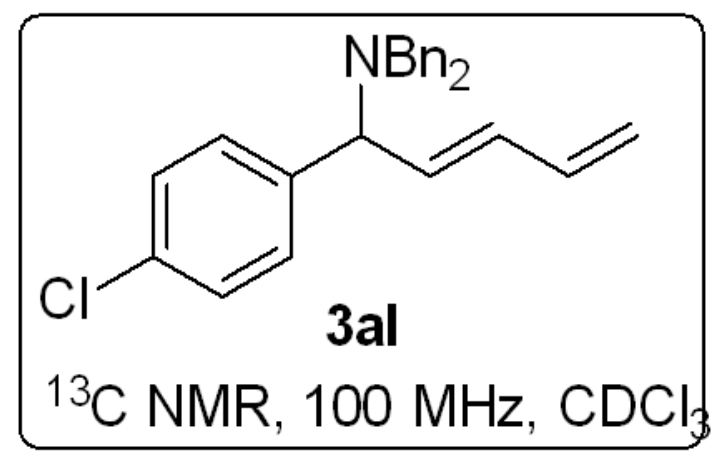



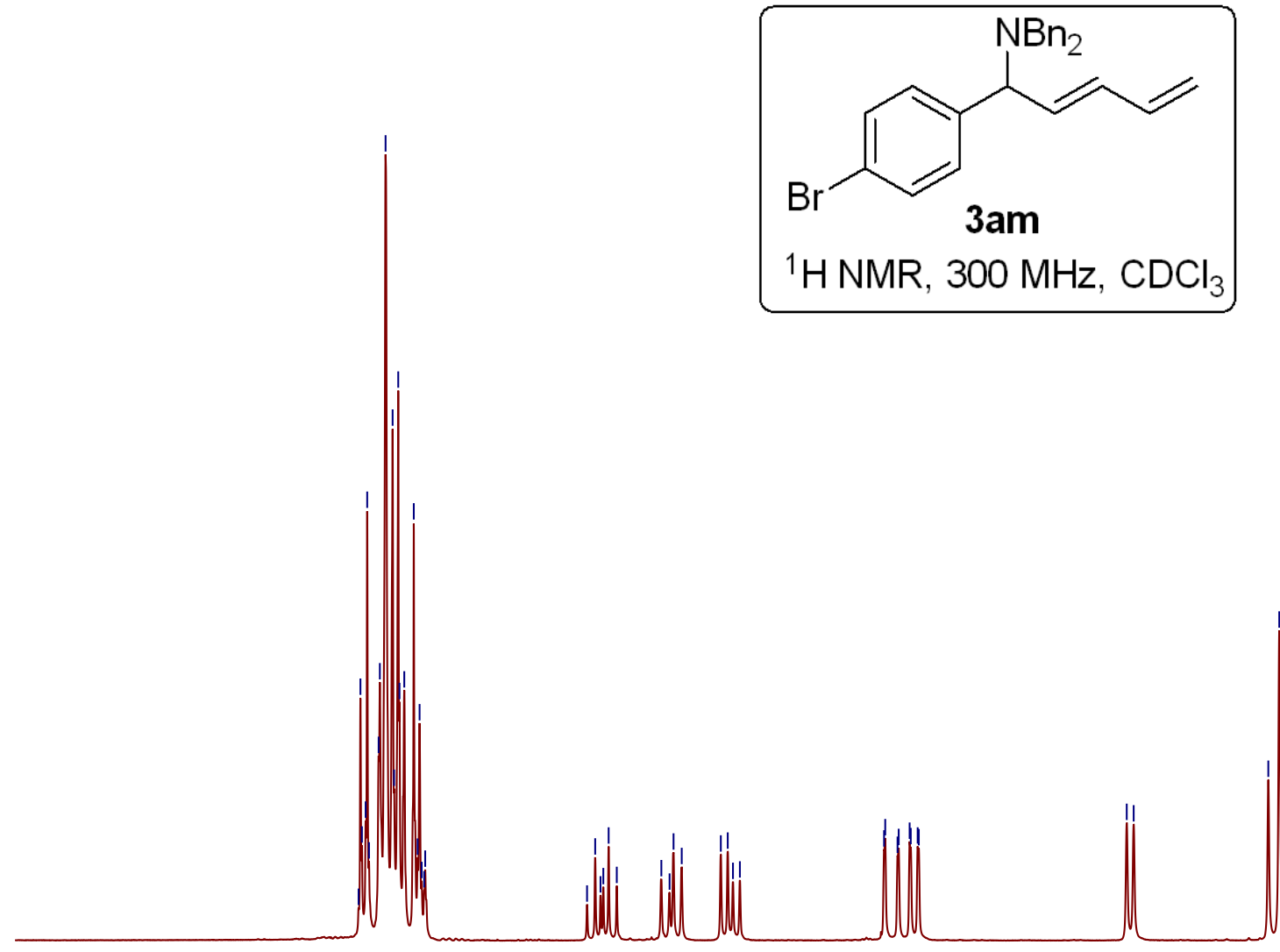

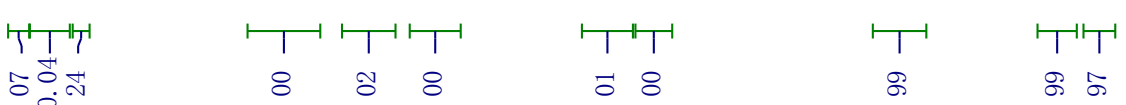

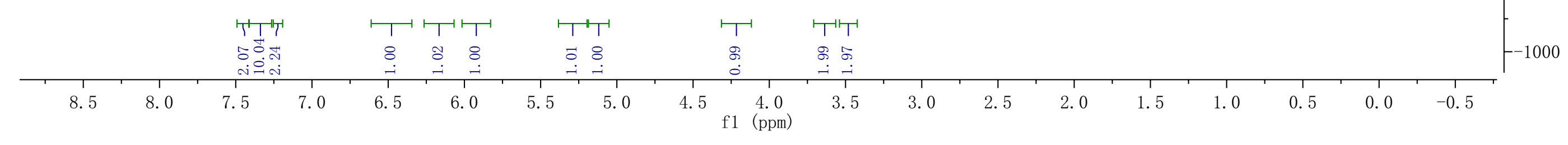




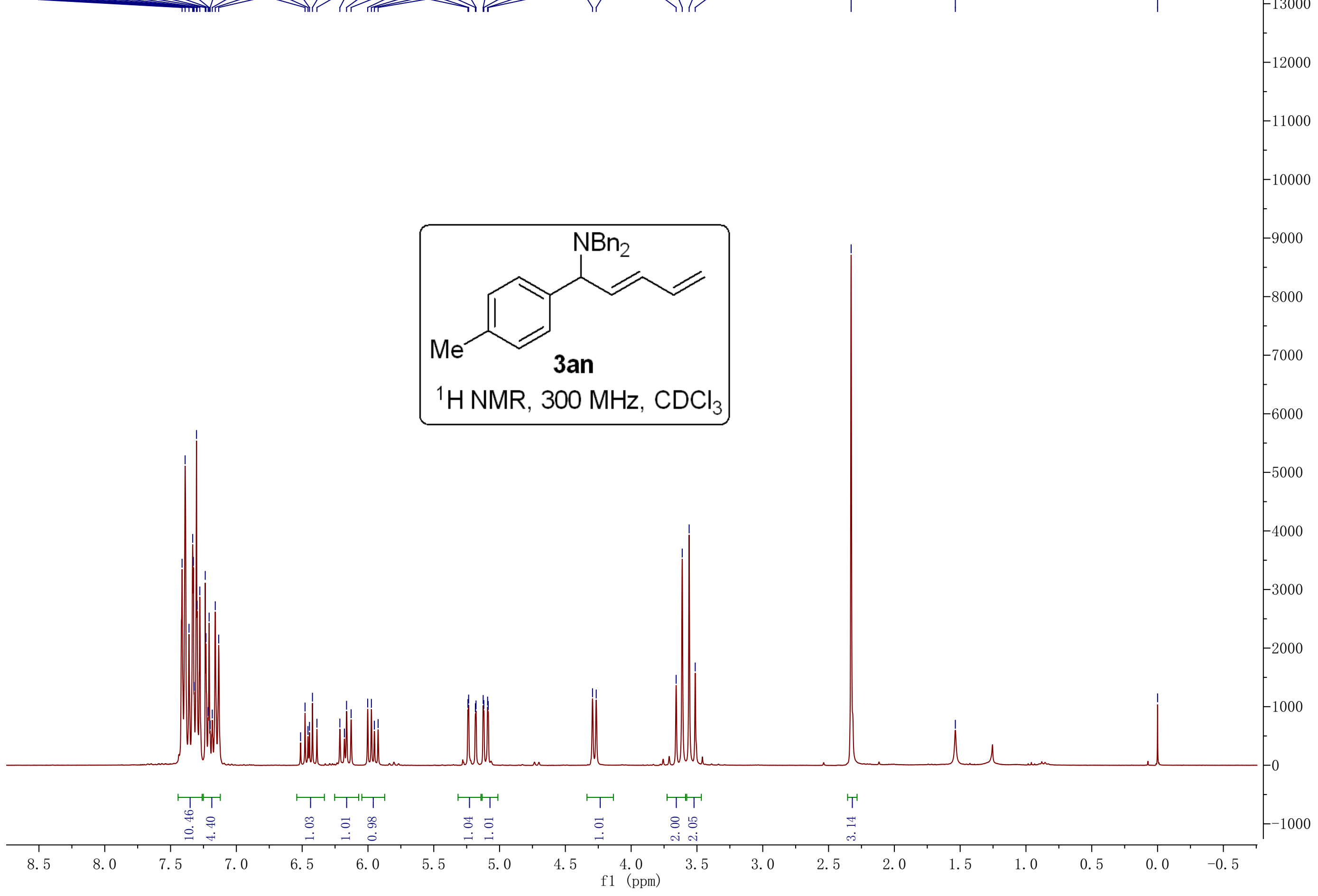




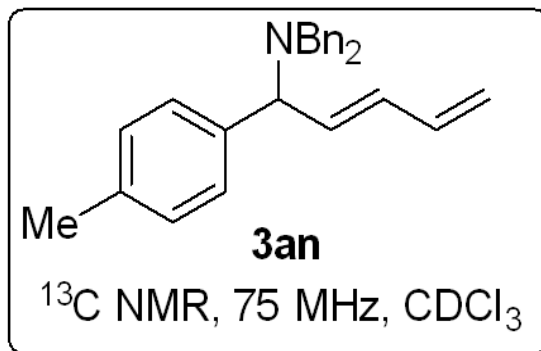




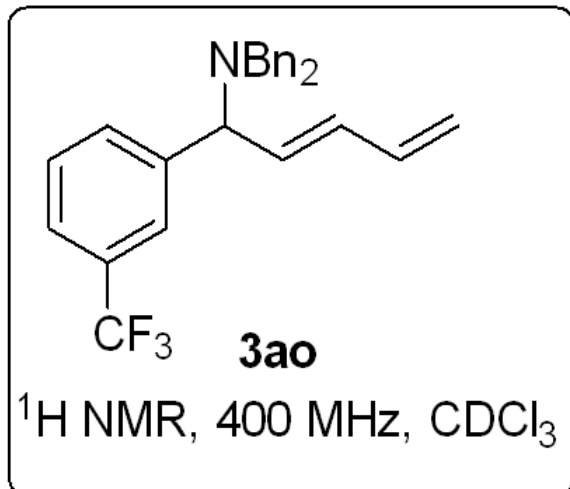




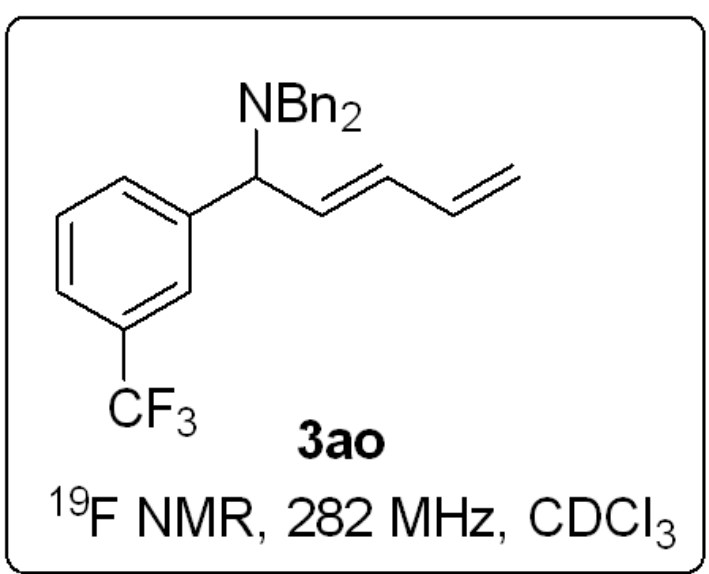




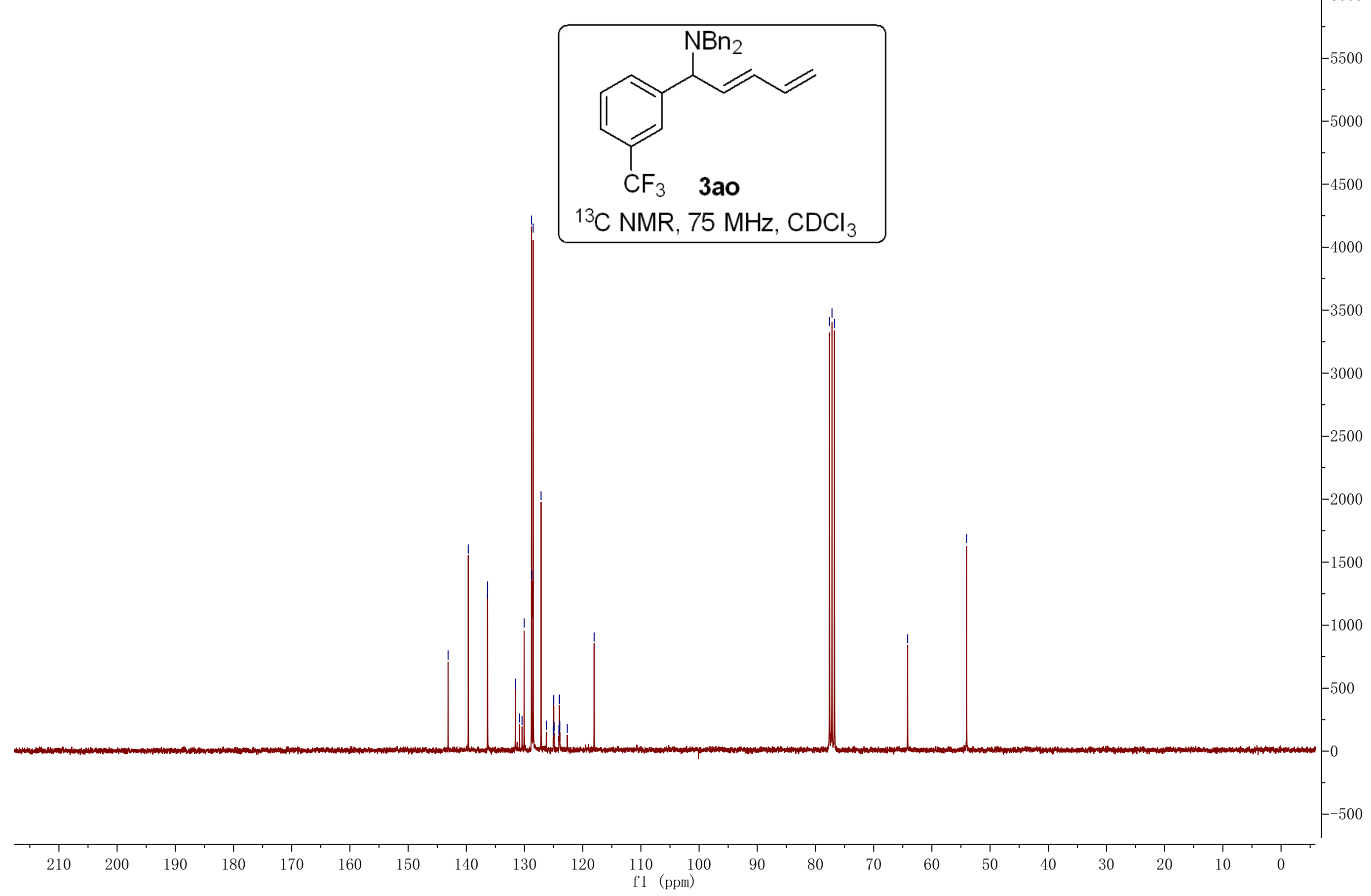




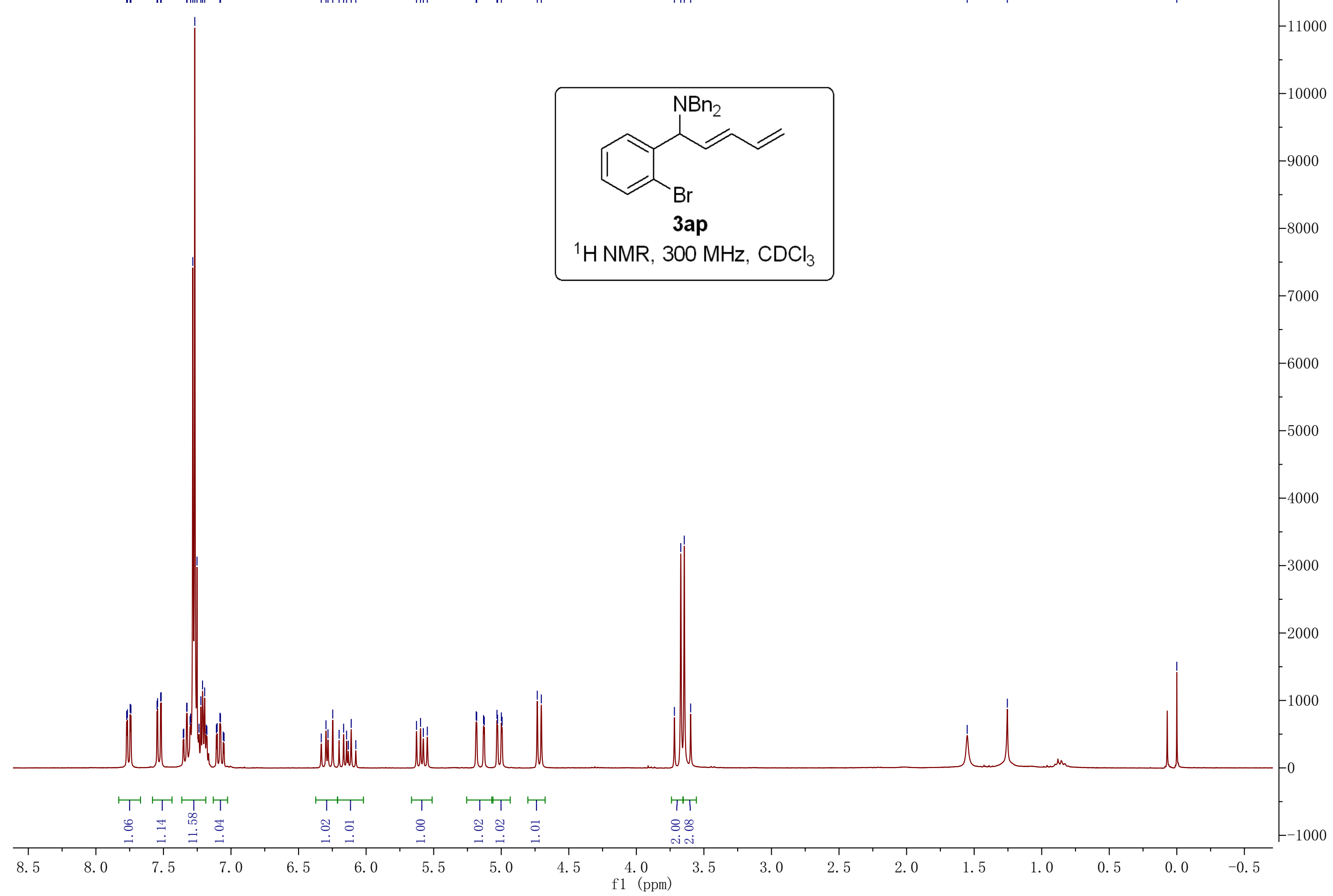




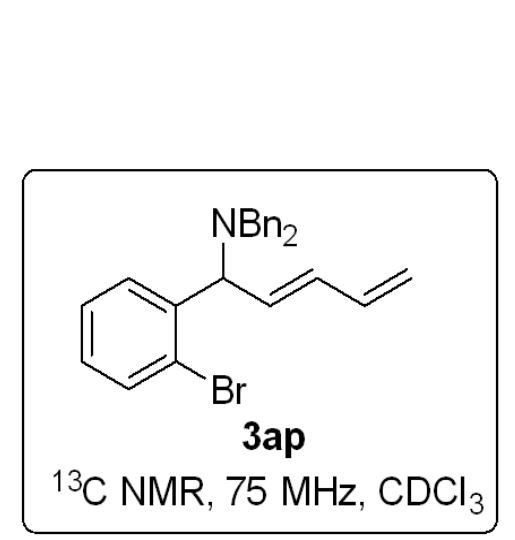

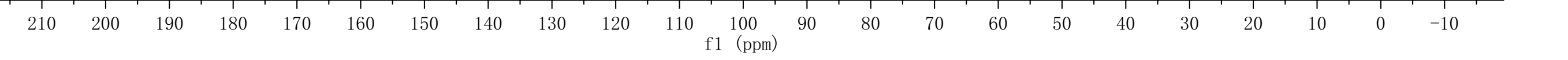




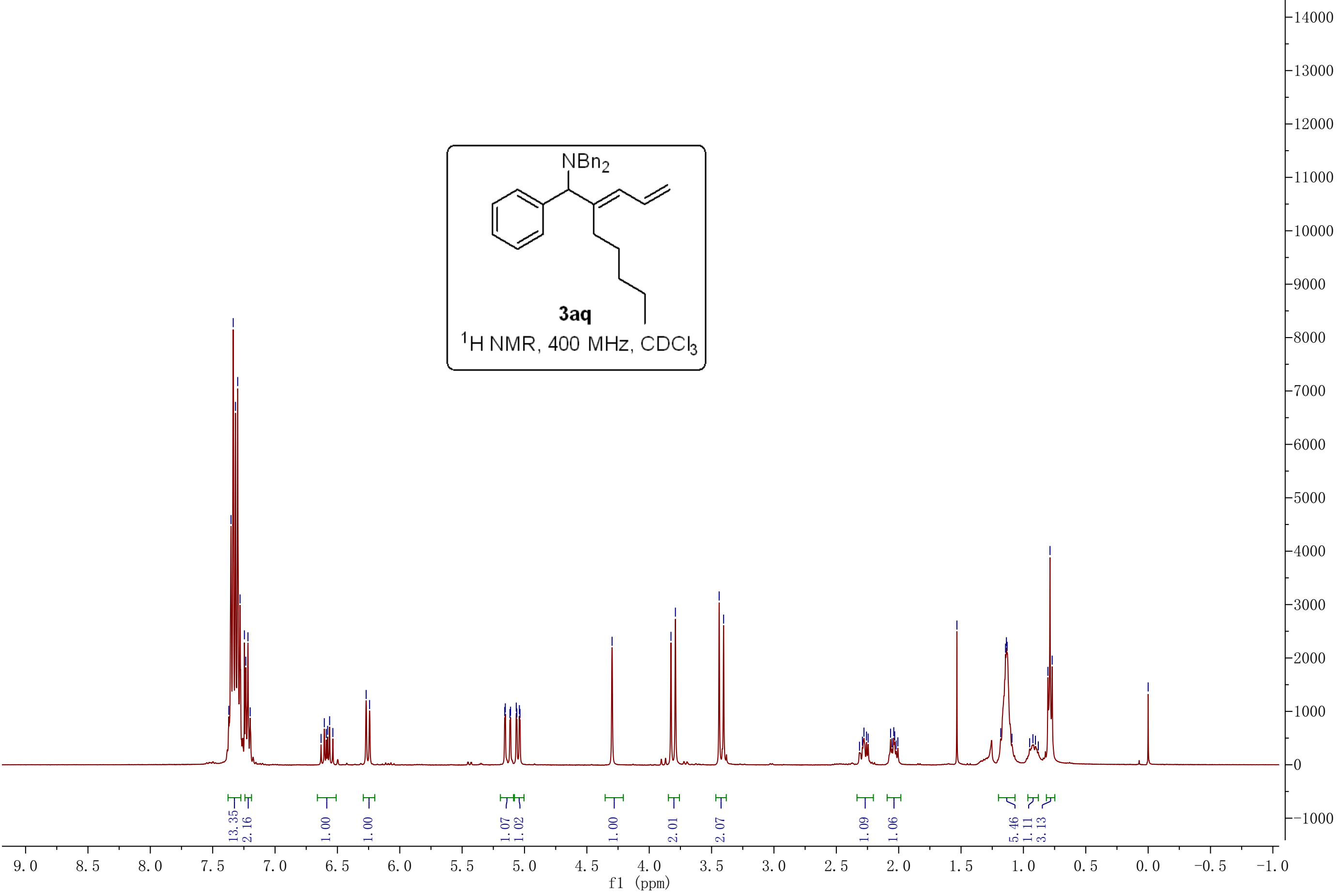



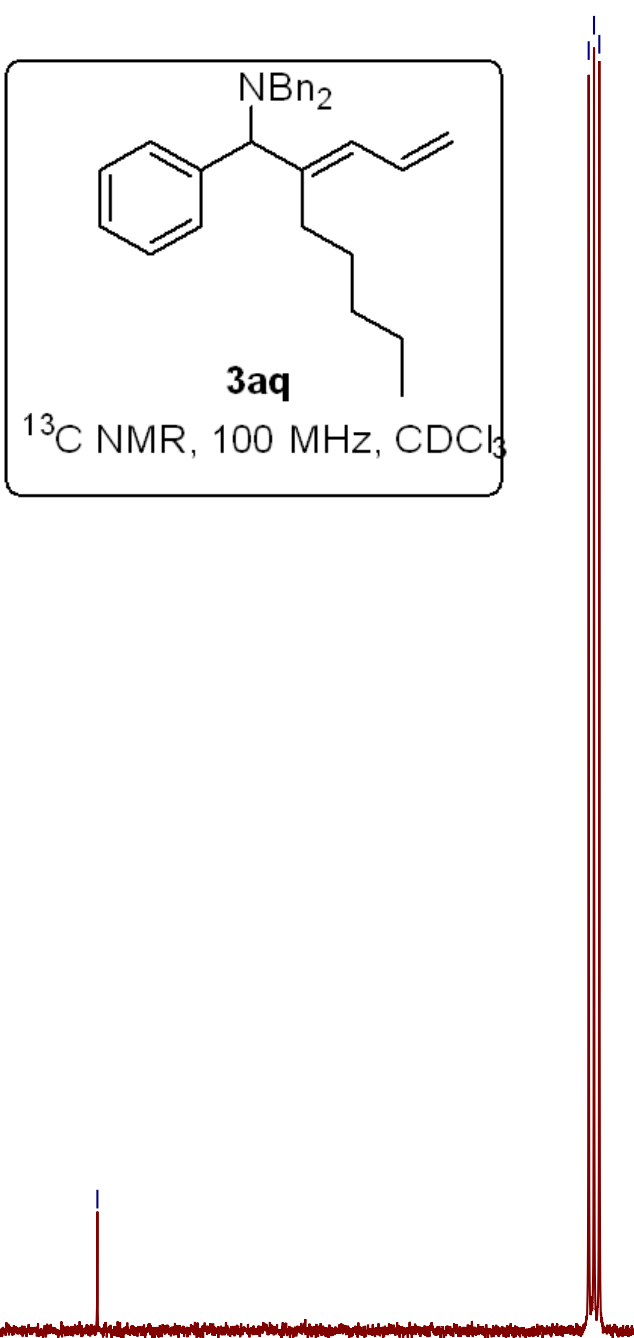

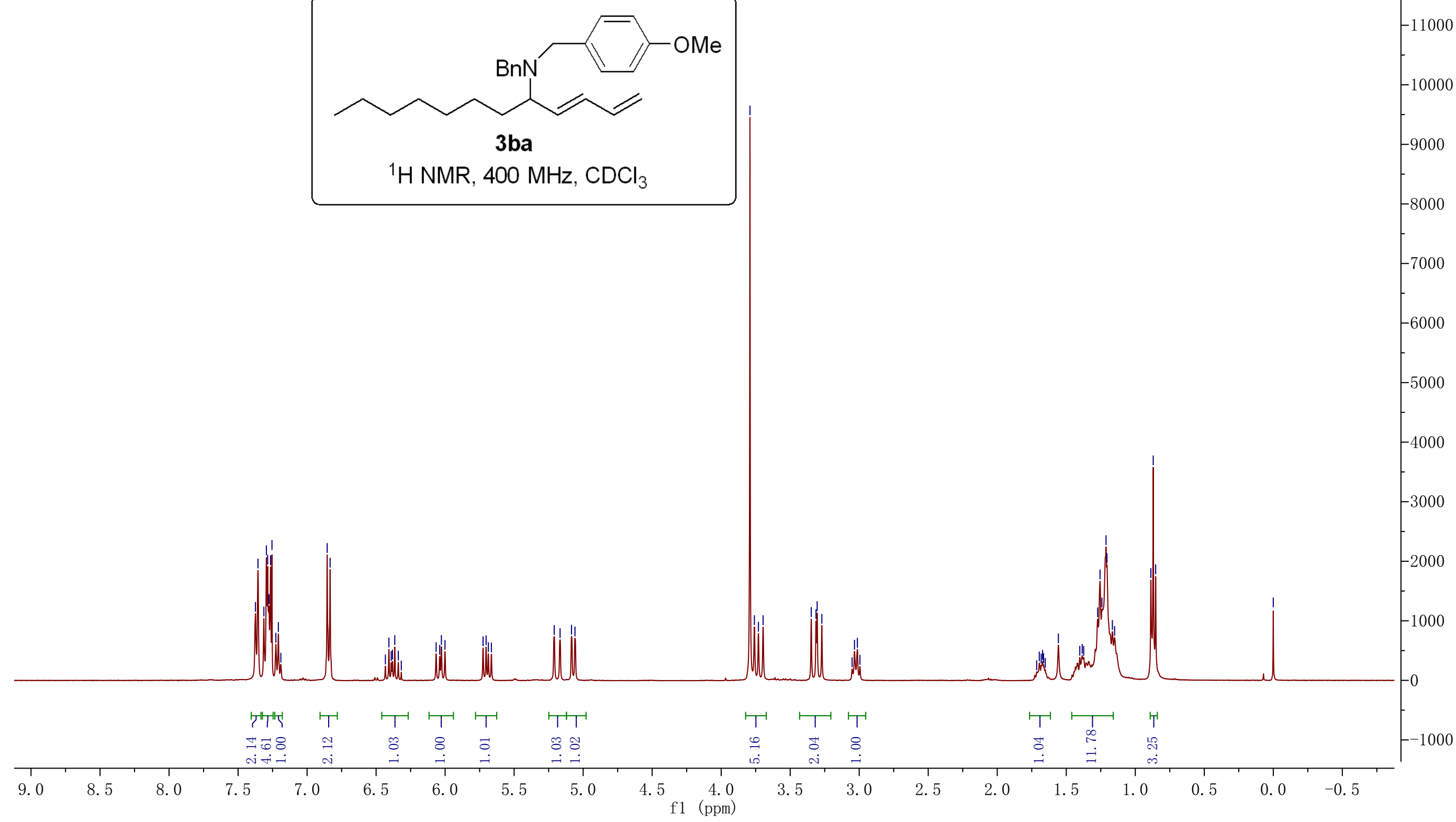

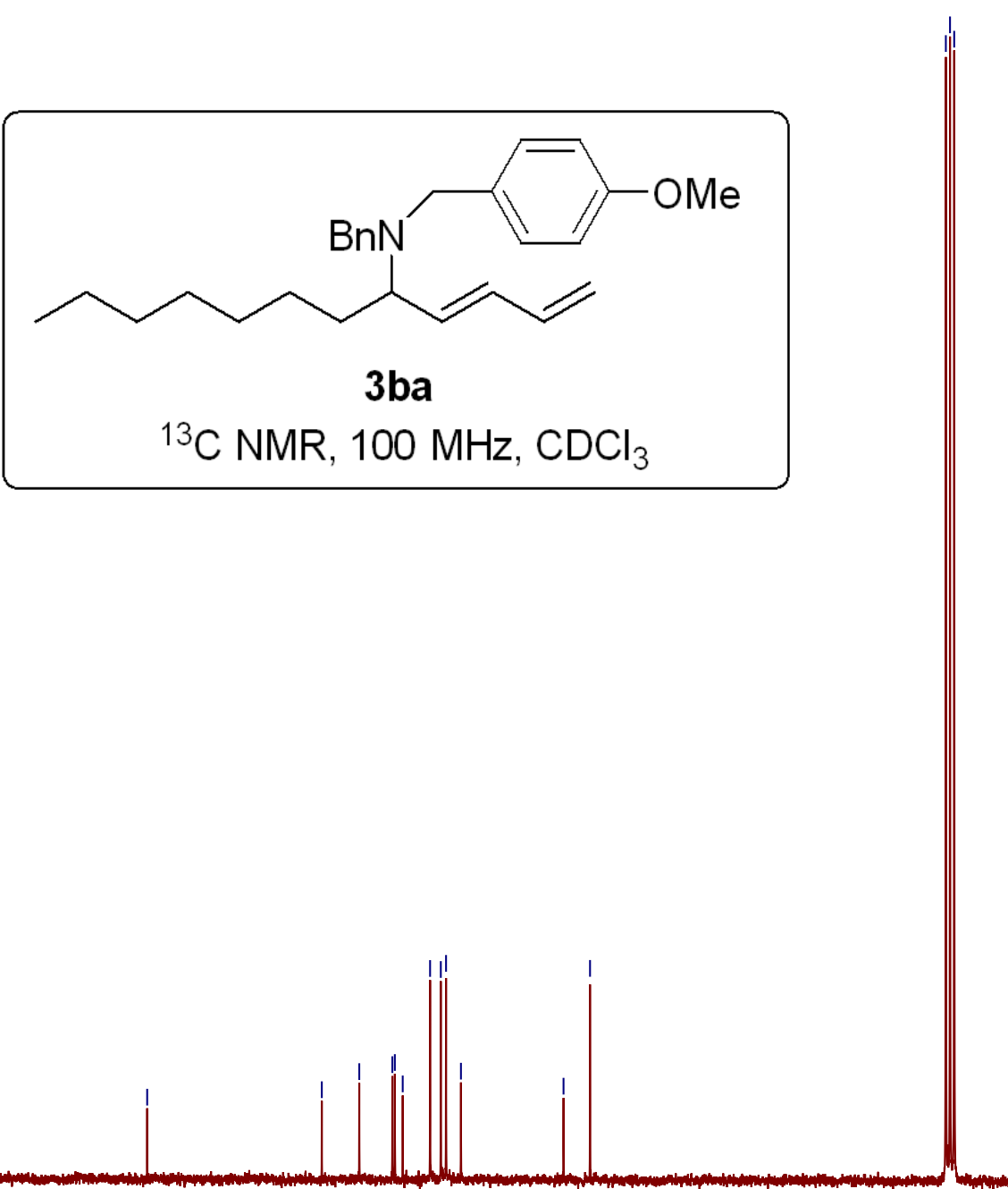


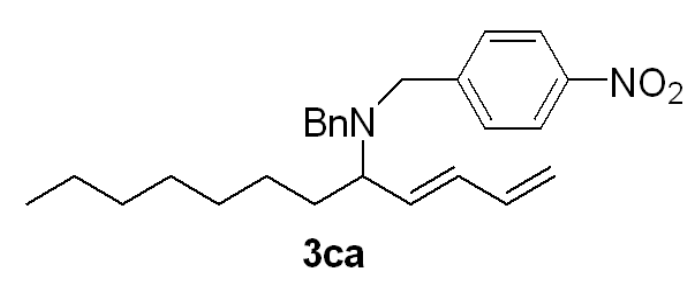

${ }^{1} \mathrm{H} \mathrm{NMR}, 400 \mathrm{MHz}, \mathrm{CDCl}_{3}$

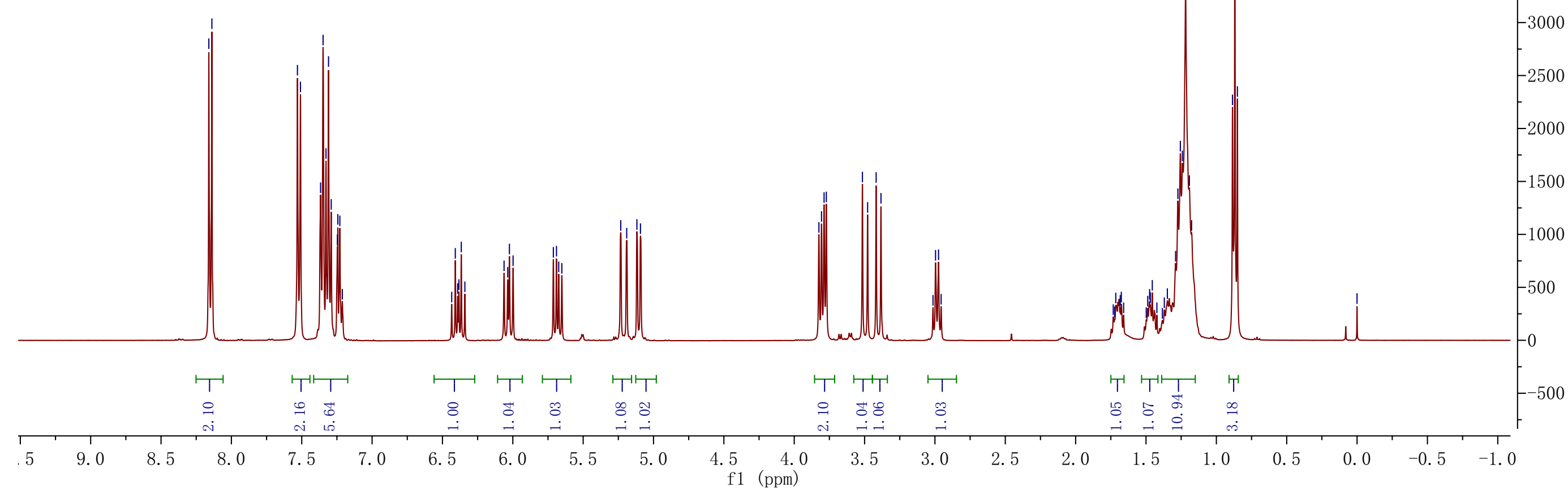



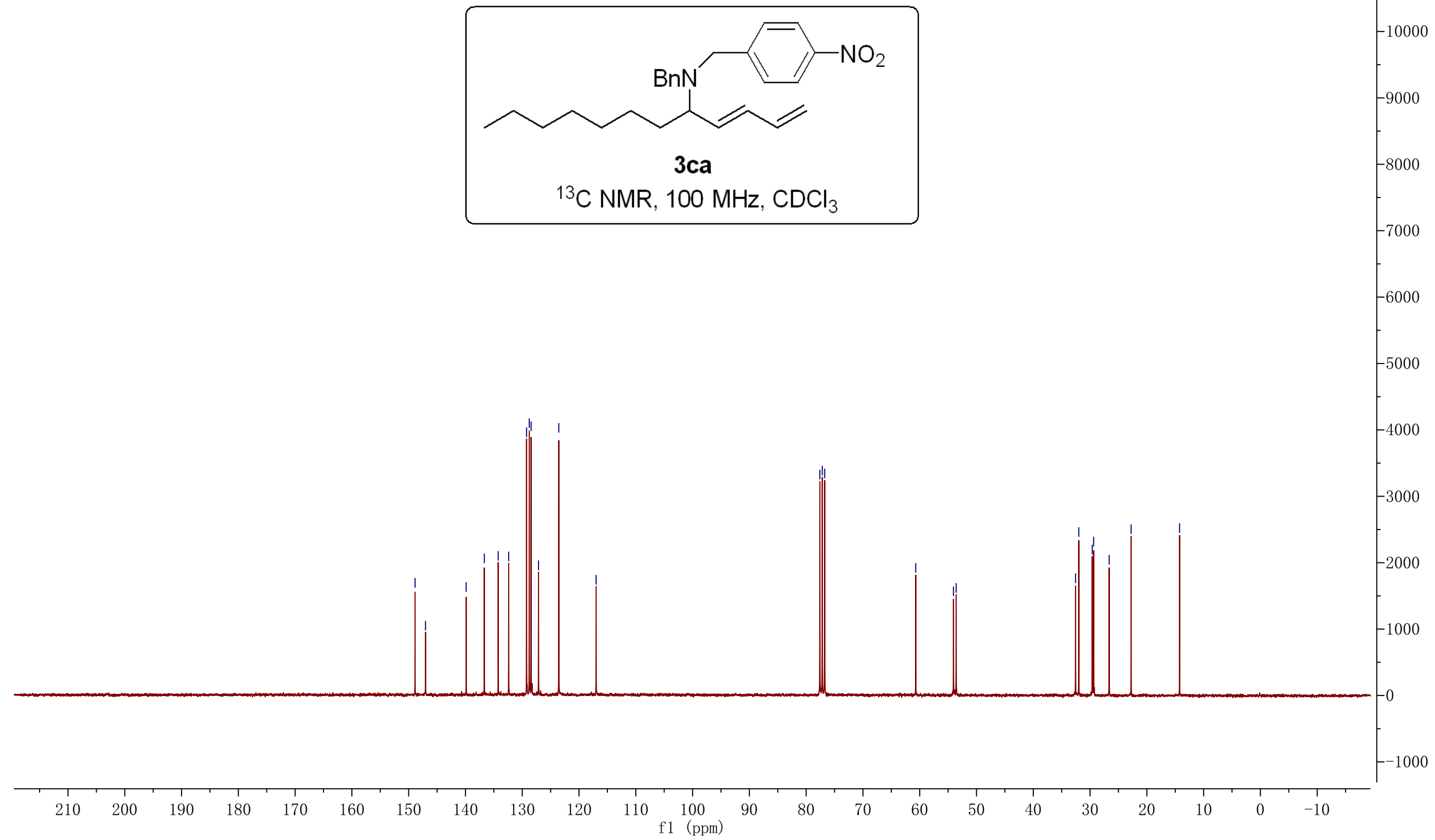


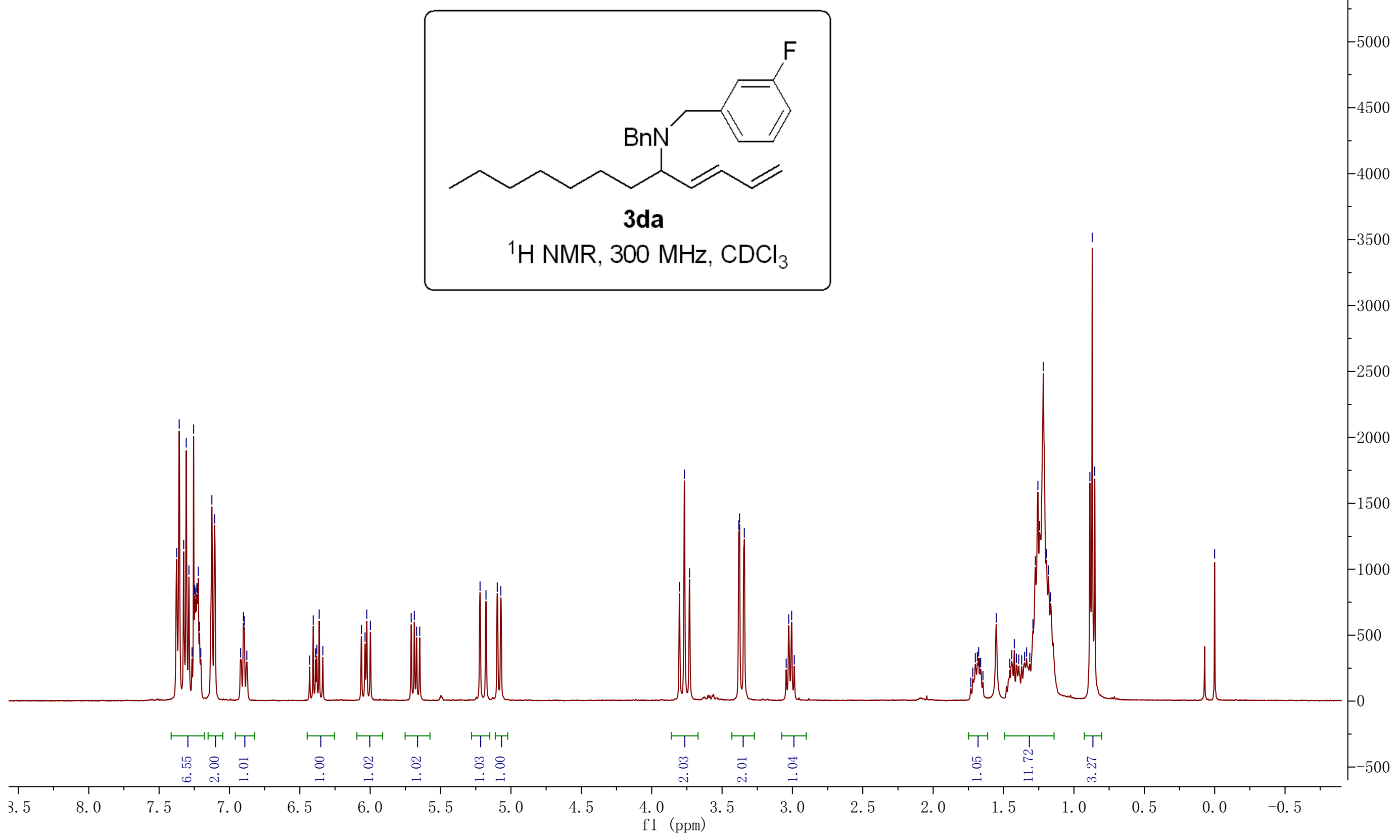




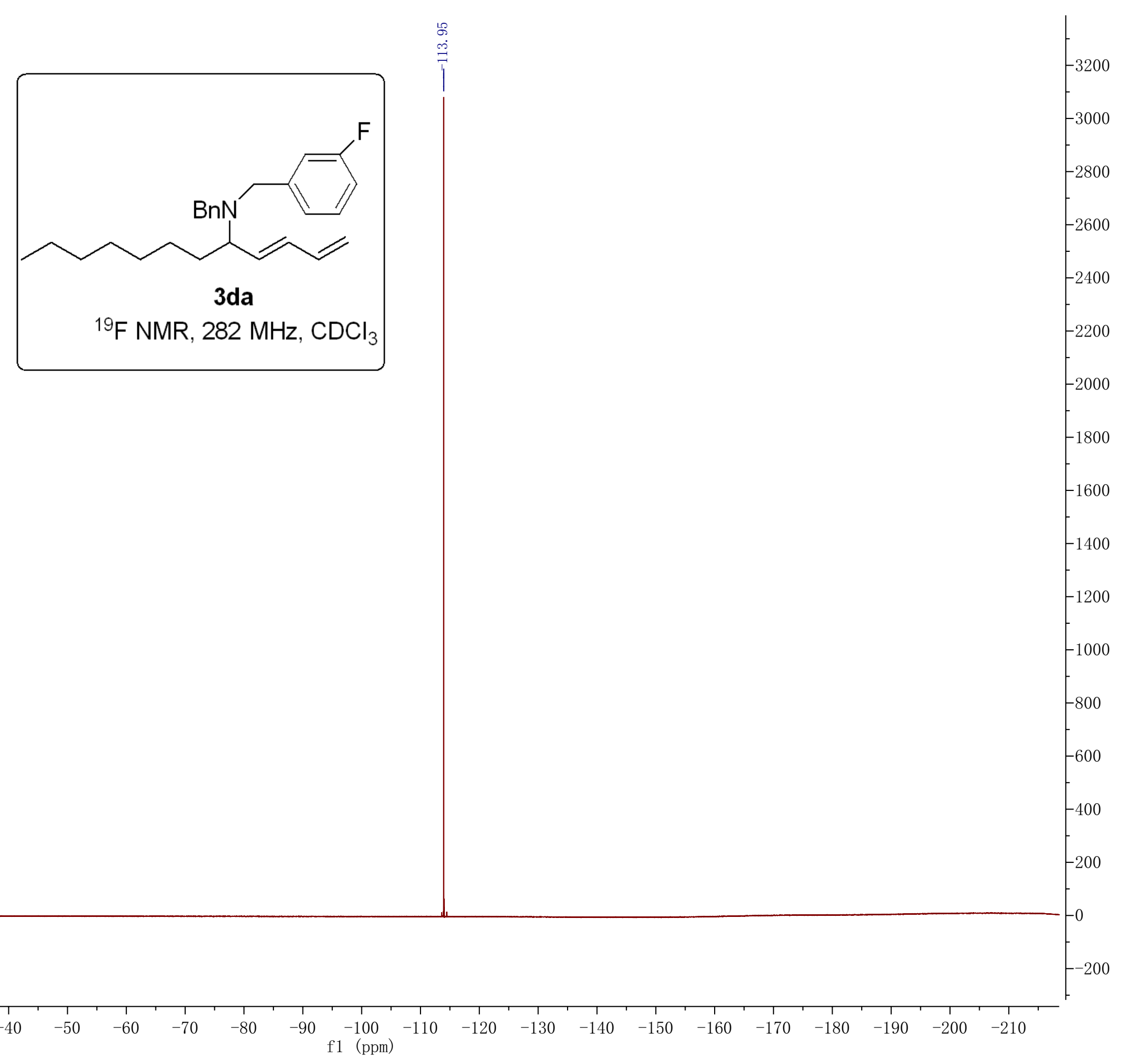



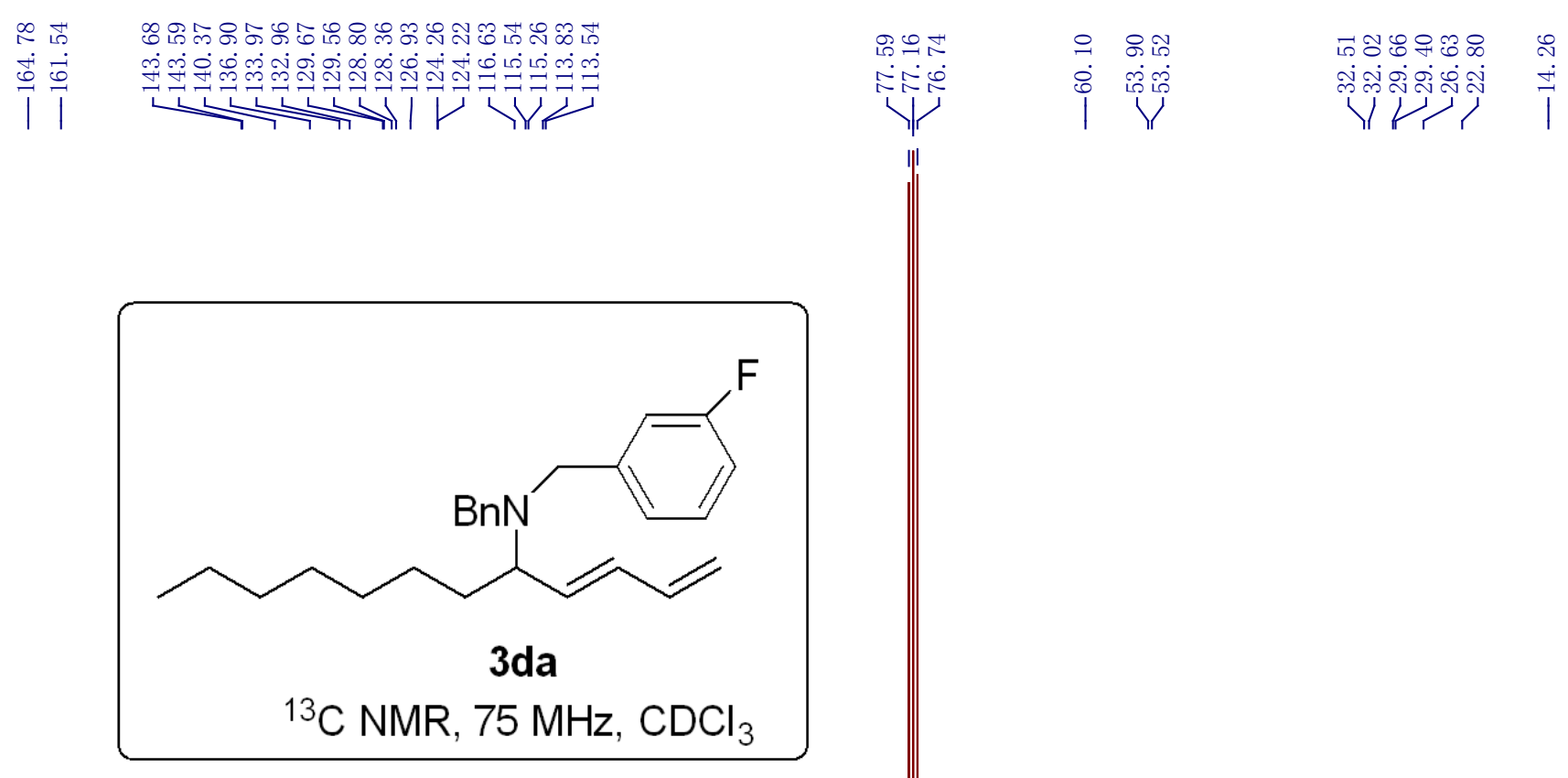


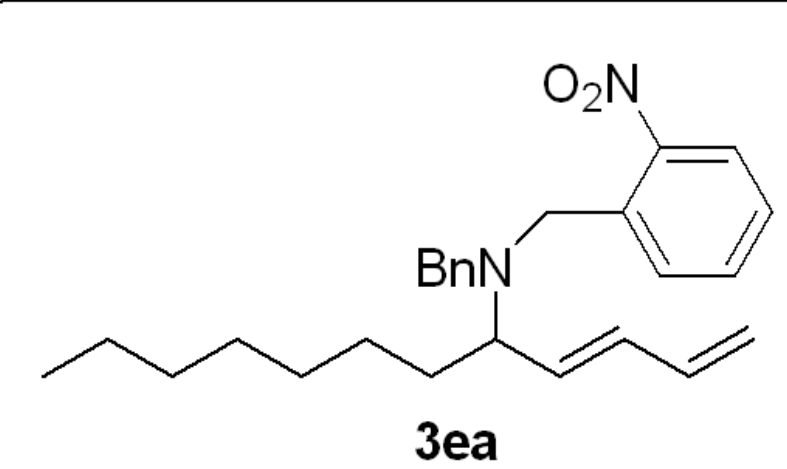

${ }^{1} \mathrm{H} \mathrm{NMR}, 400 \mathrm{MHz}, \mathrm{CDCl}_{3}$
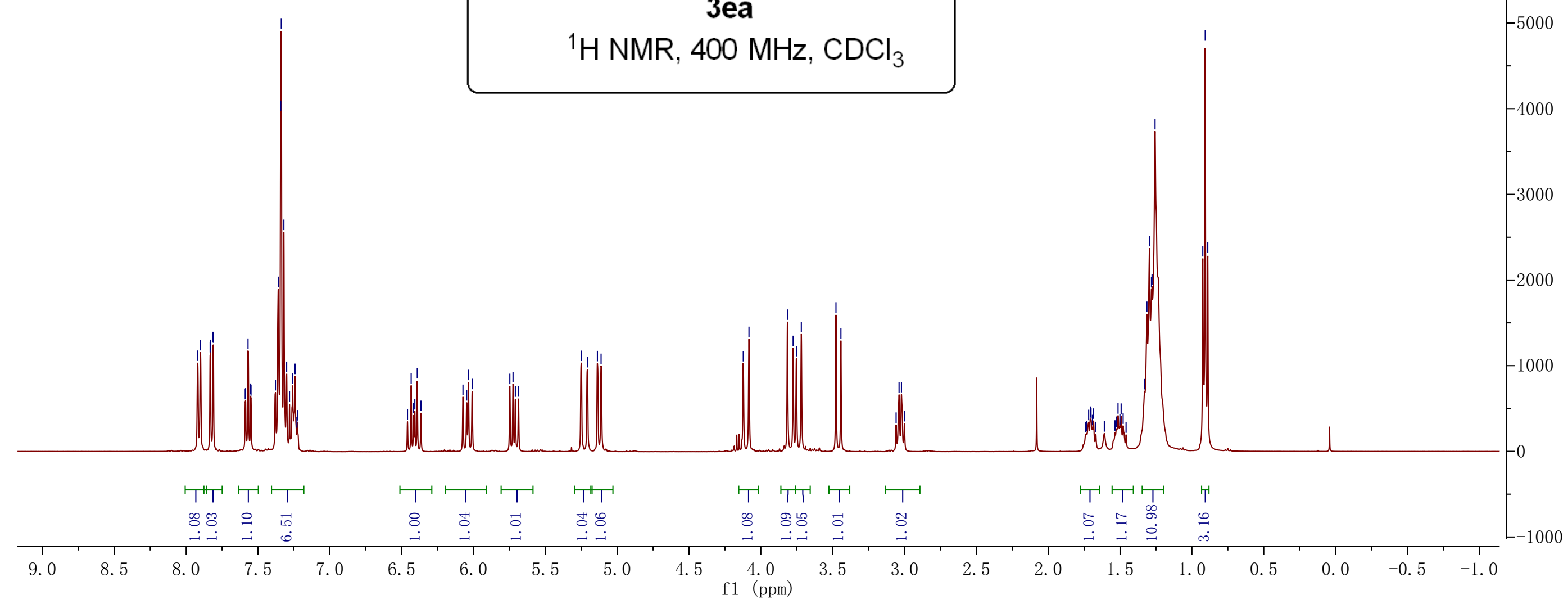

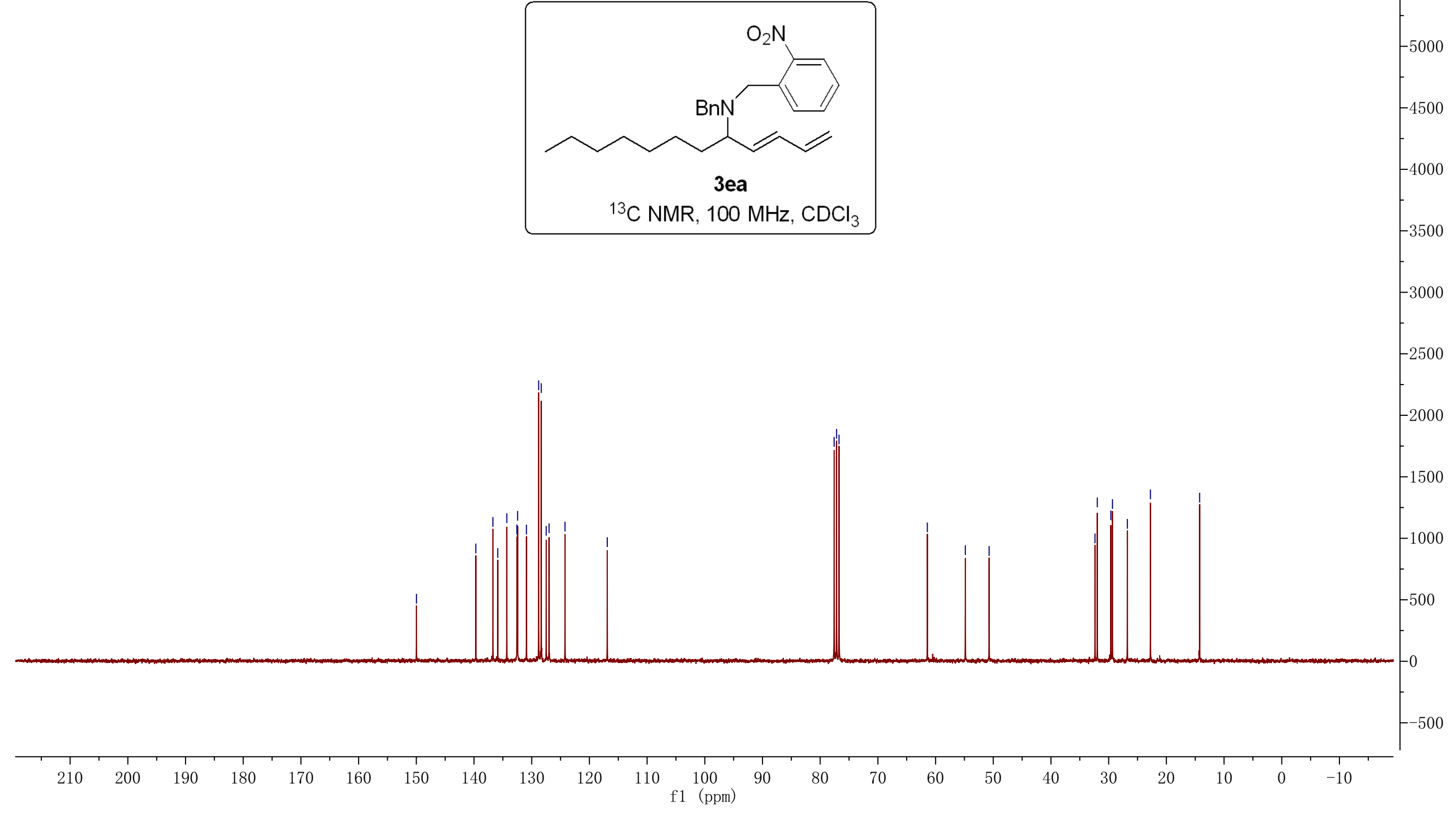


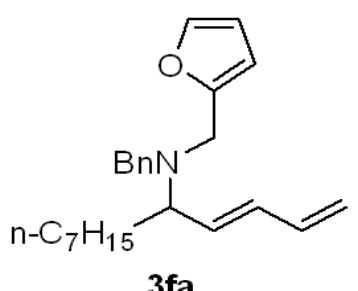

${ }^{1} \mathrm{HNMR}, 400 \mathrm{MHz}, \mathrm{CDCl}_{3}$

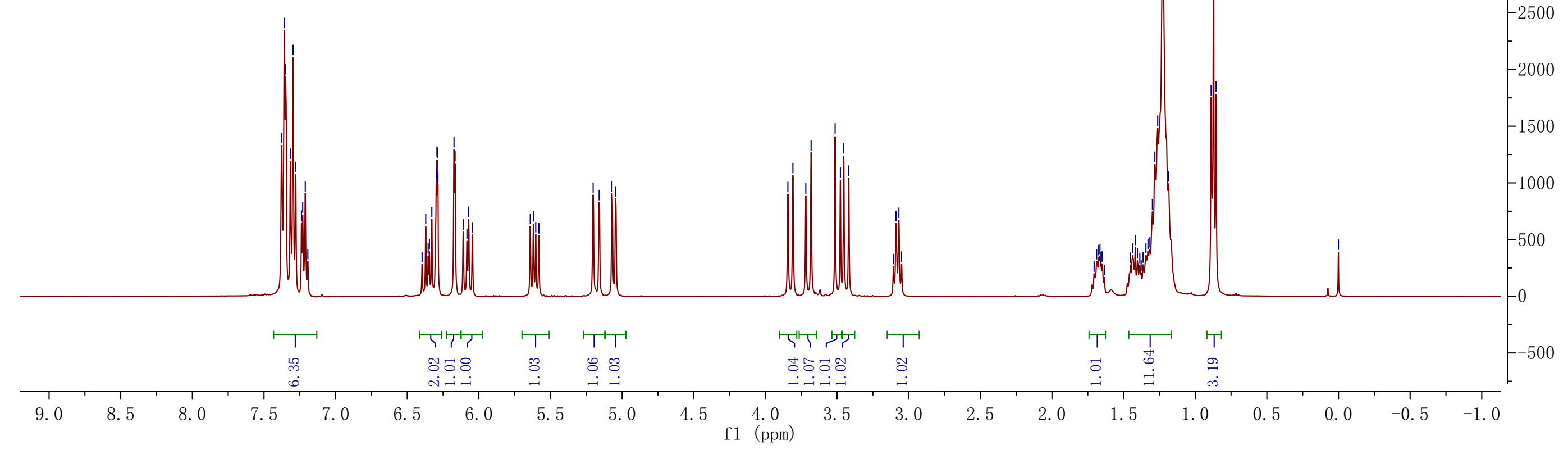




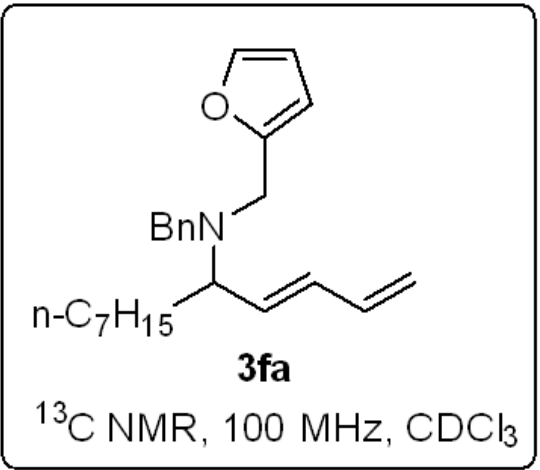



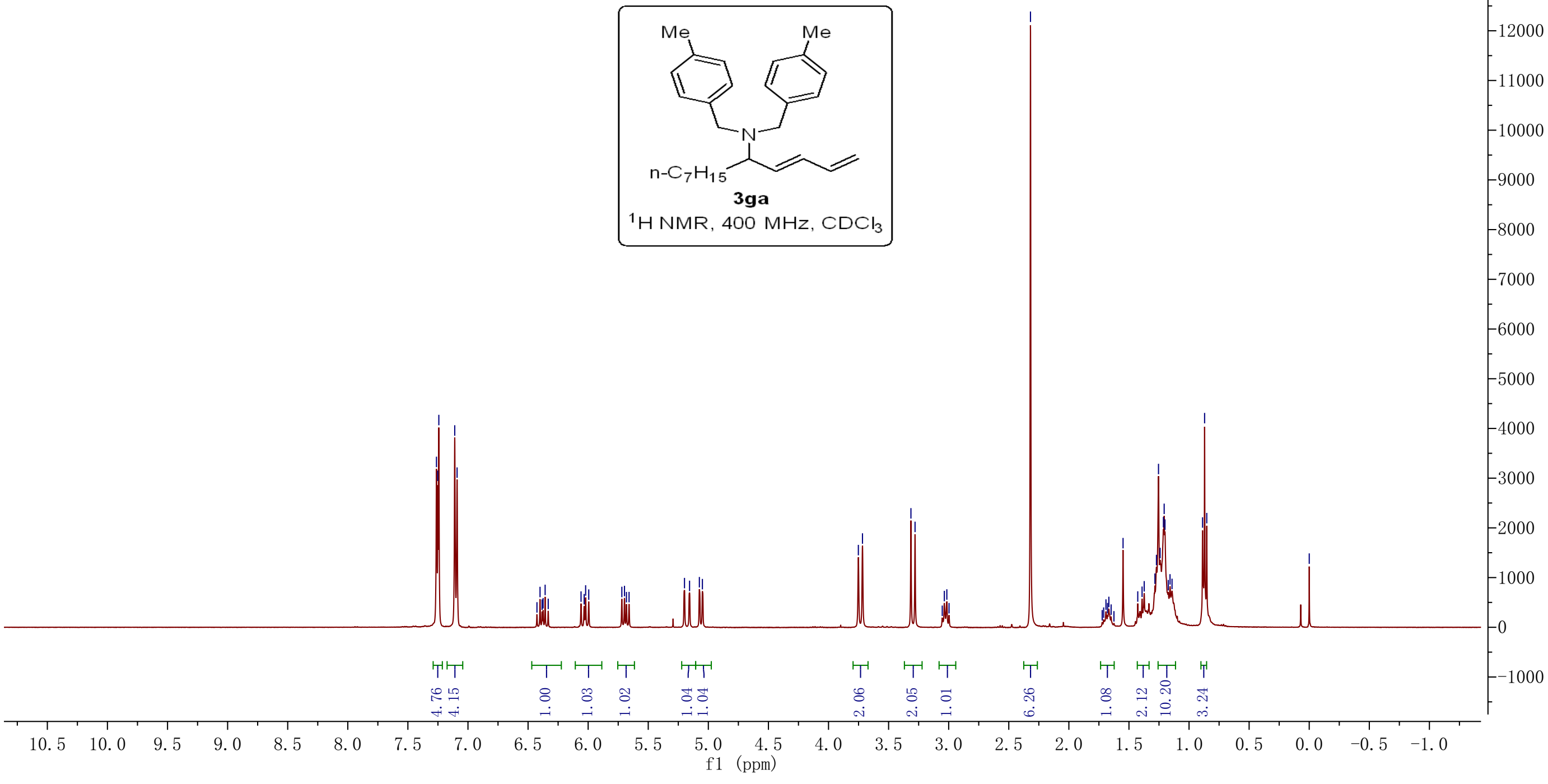


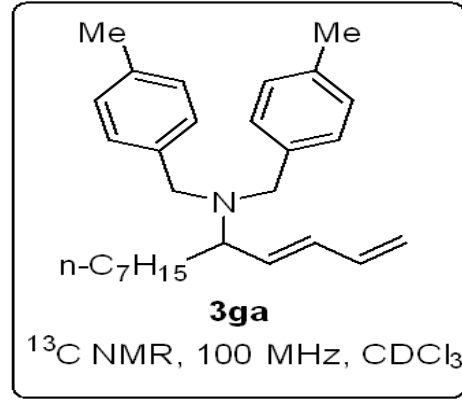




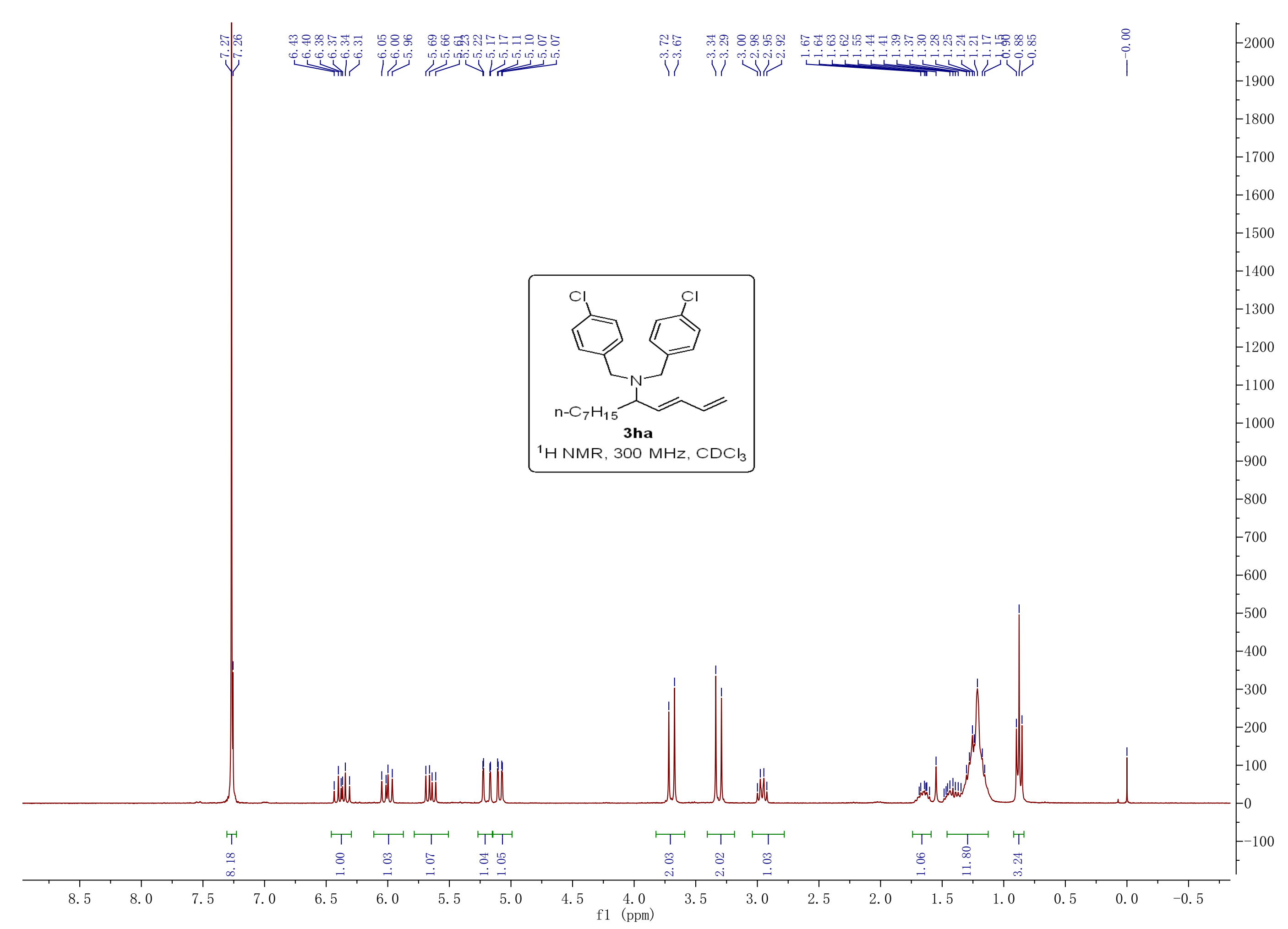



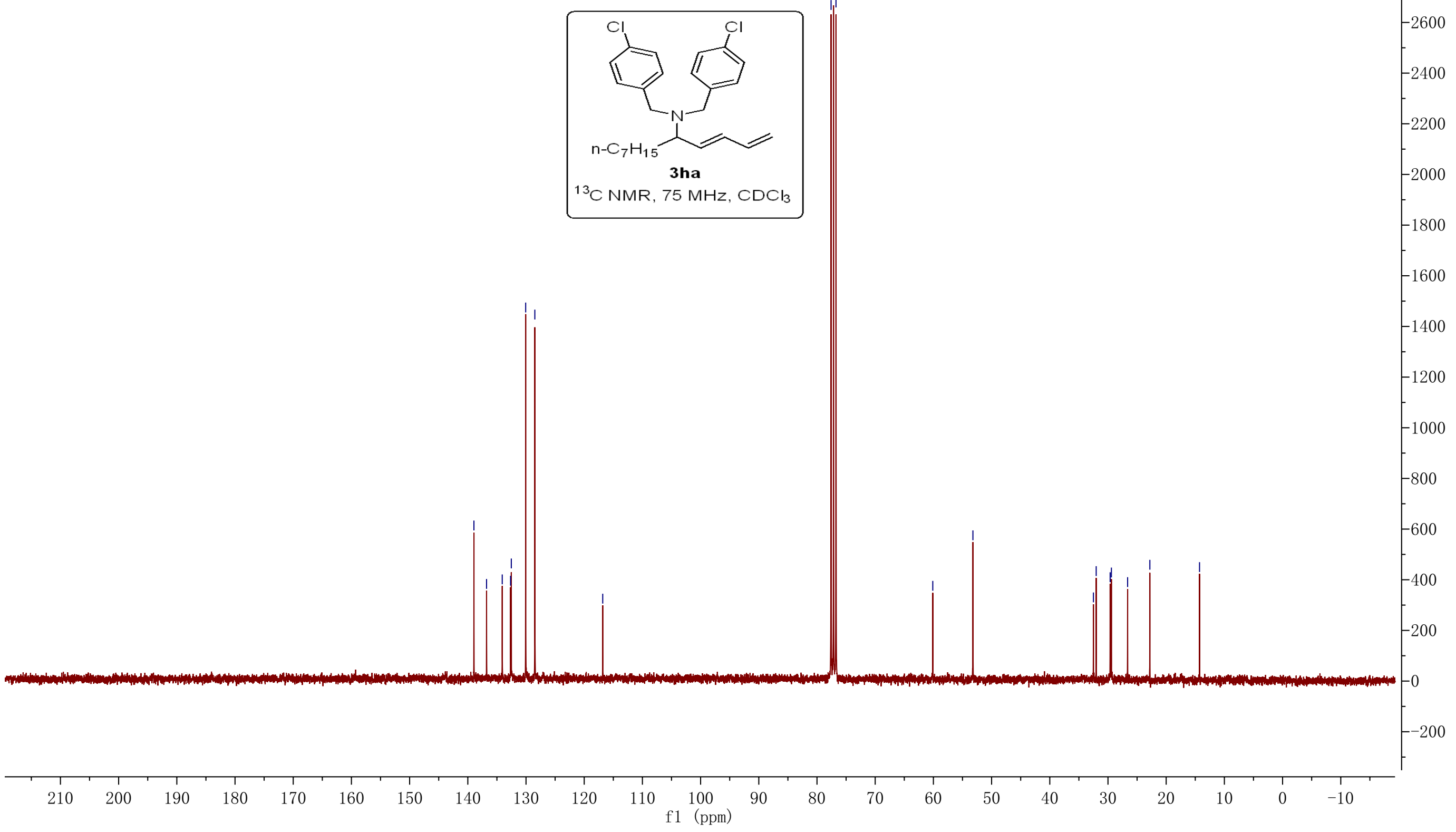

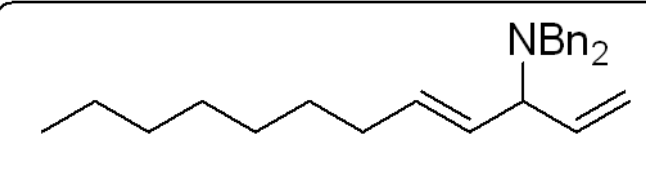

$4 a a$

${ }^{1} \mathrm{H} \mathrm{NMR}, 400 \mathrm{MHz}, \mathrm{CDCl}_{3}$

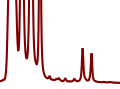

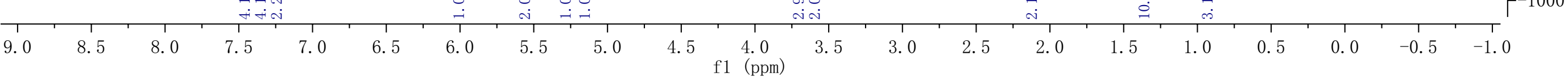



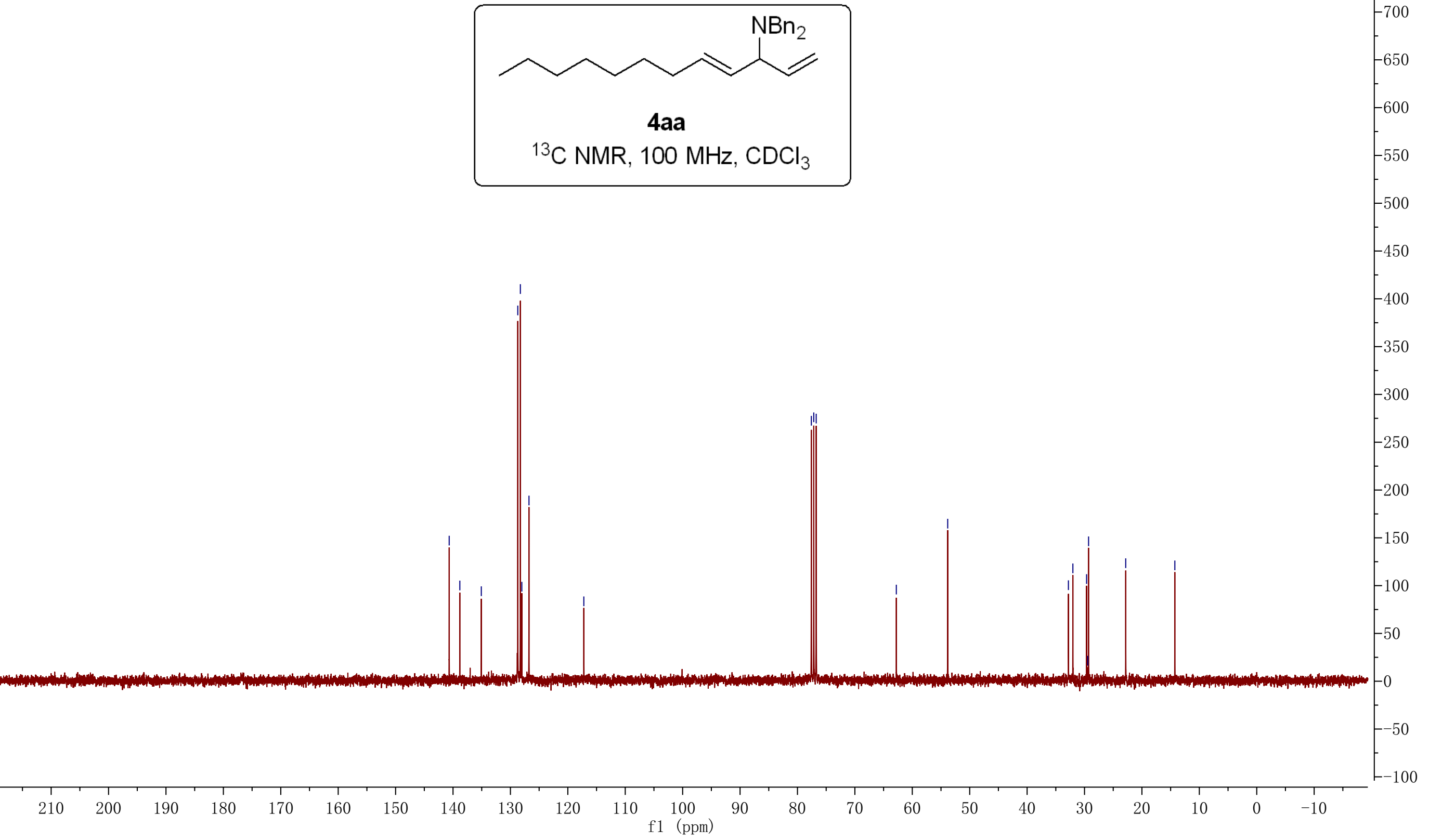

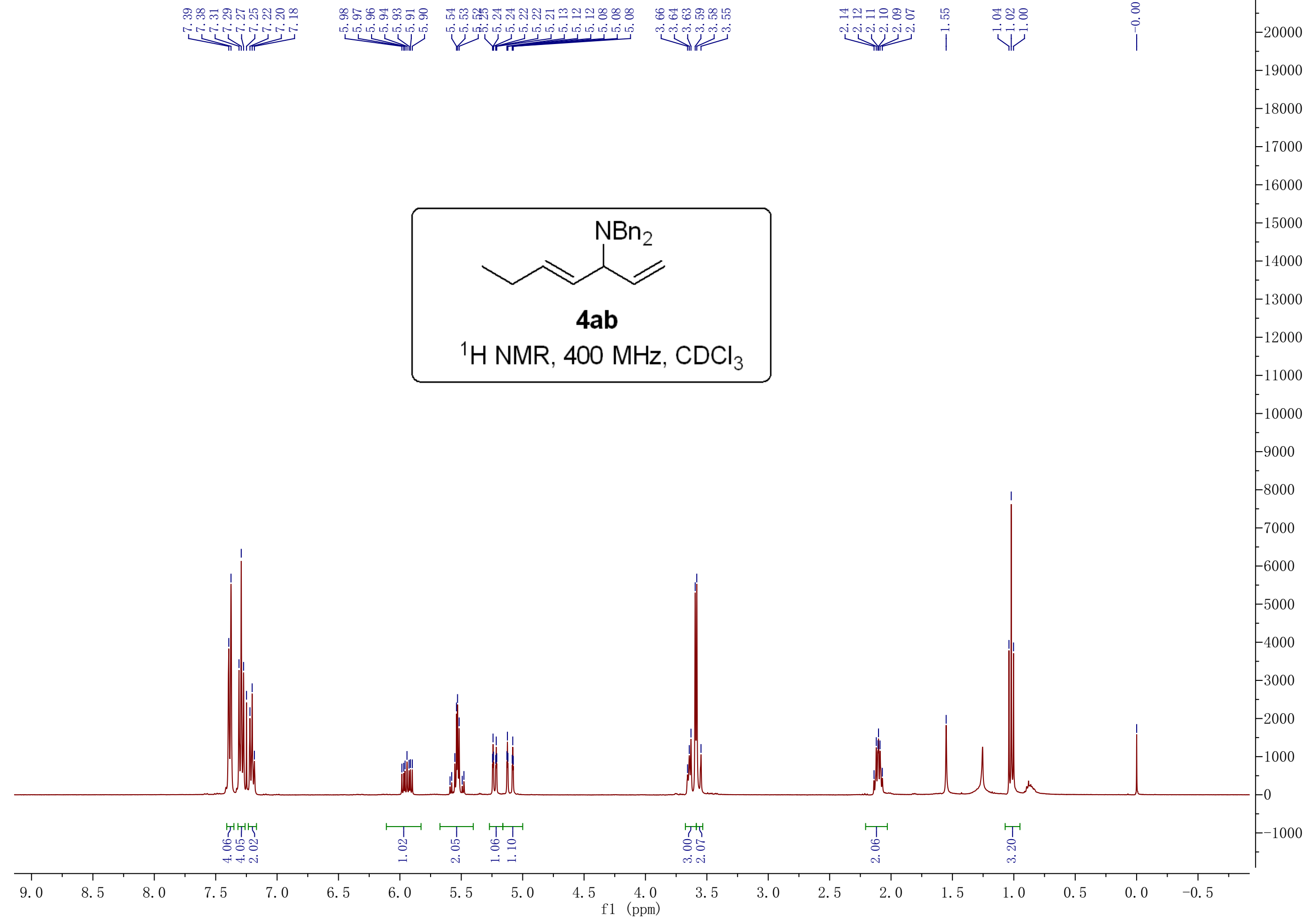

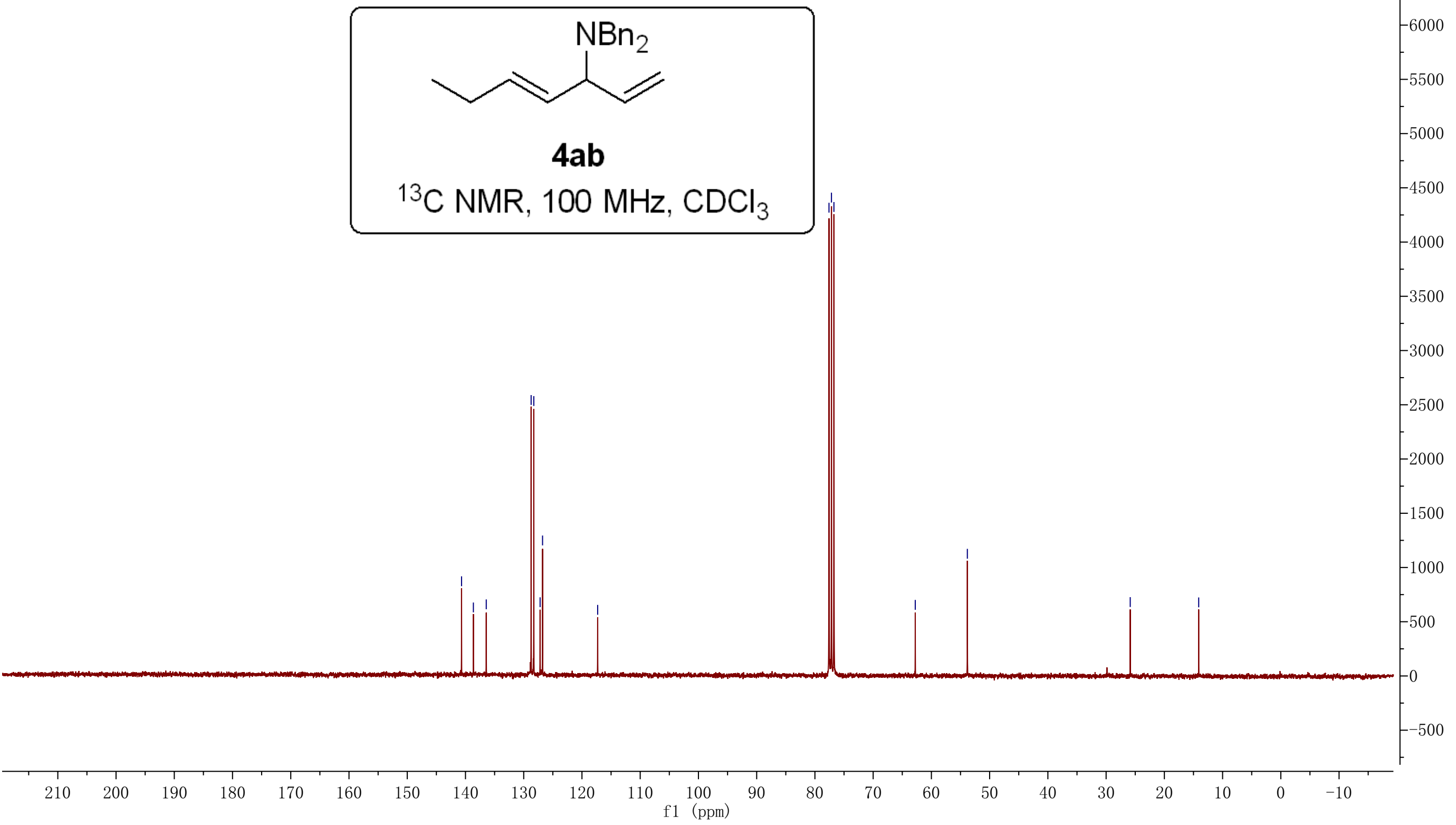

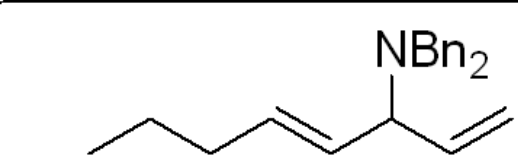

$4 a c$

${ }^{1} \mathrm{H}$ NMR, $400 \mathrm{MHz}, \mathrm{CDCl}_{3}$

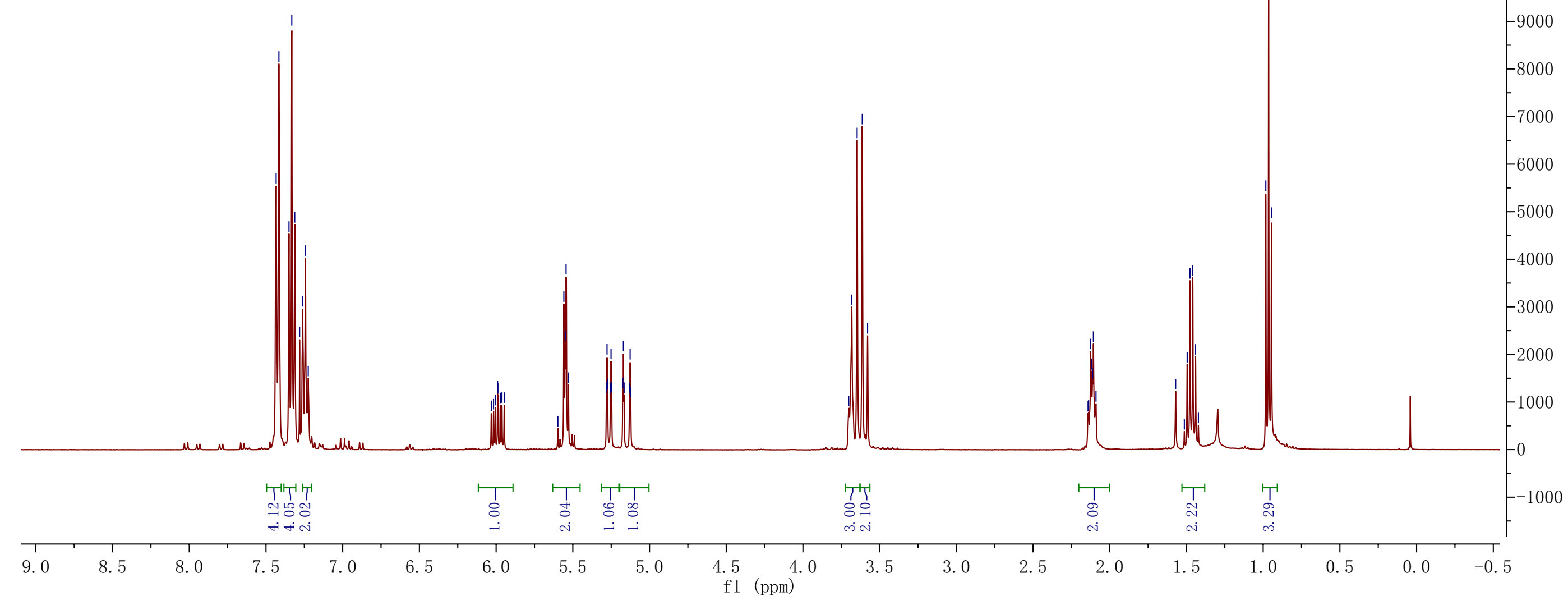



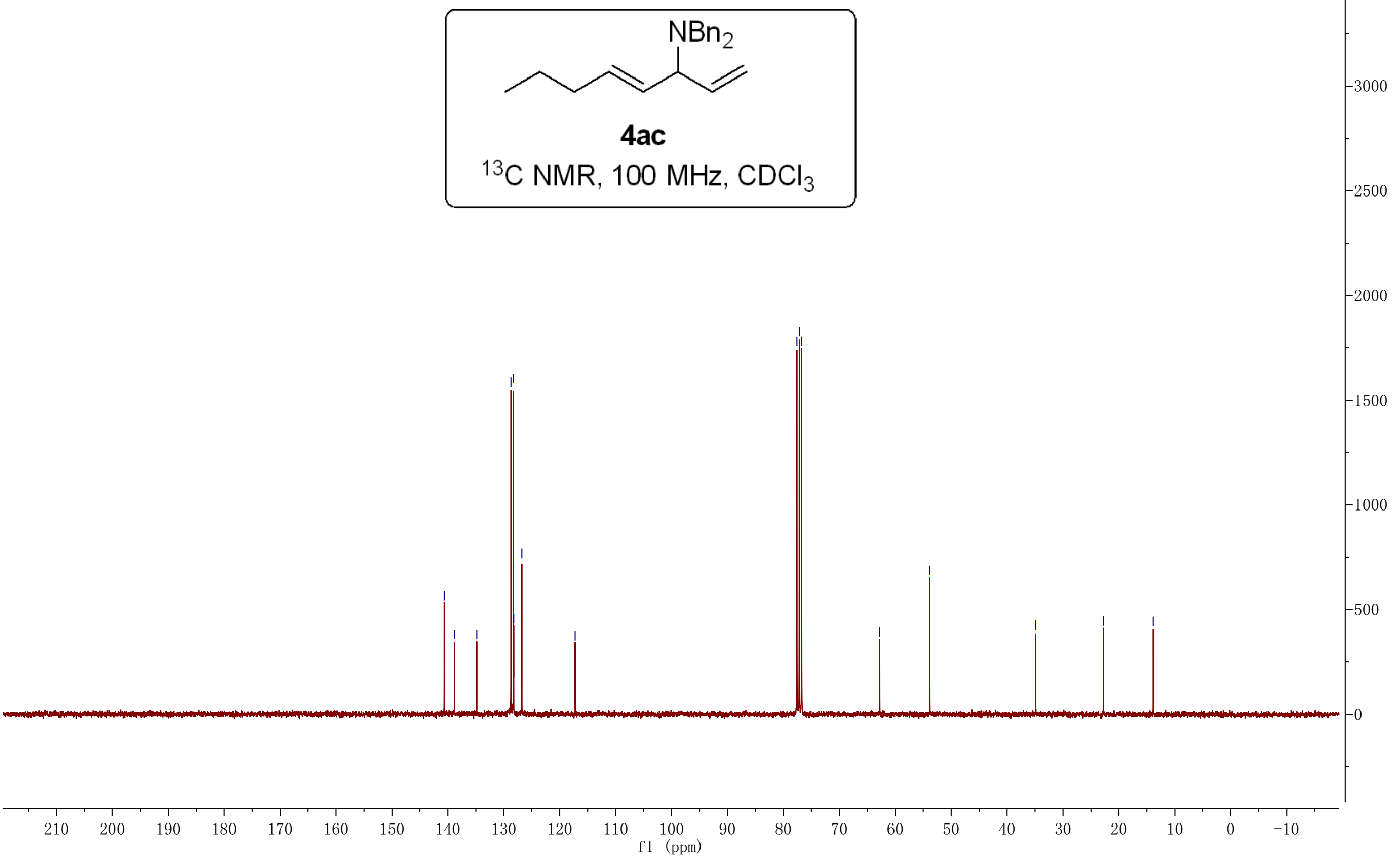

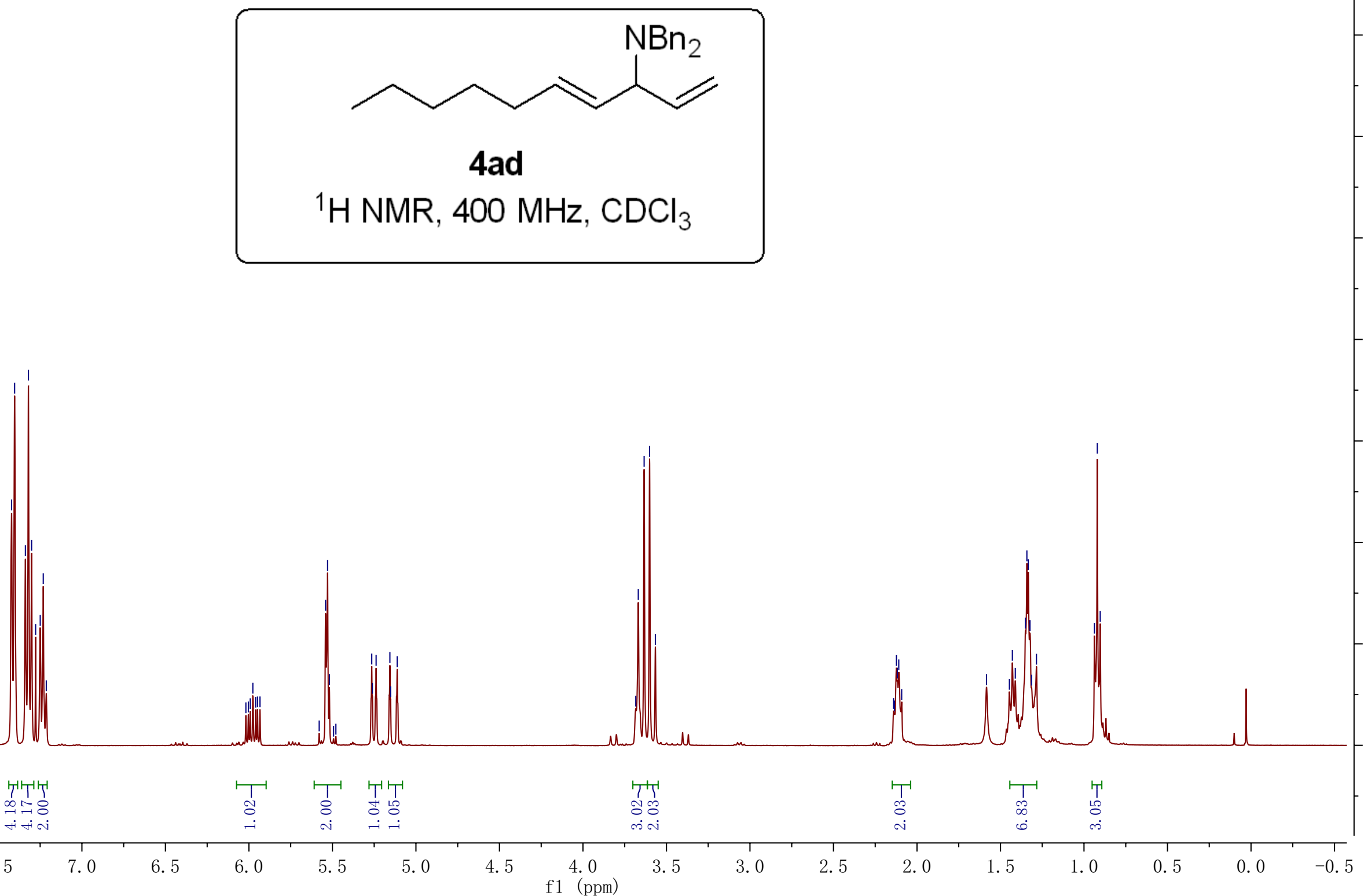


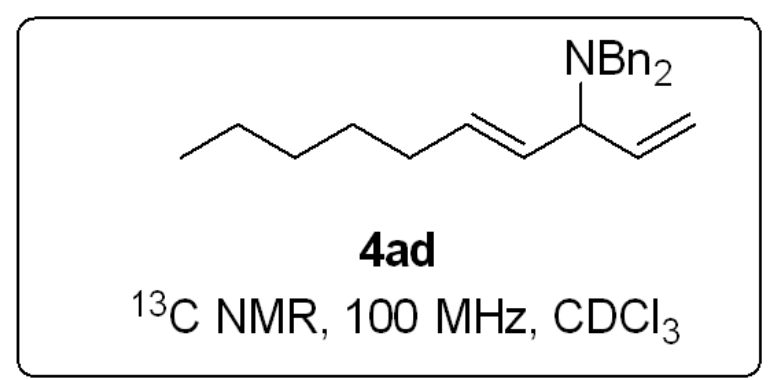



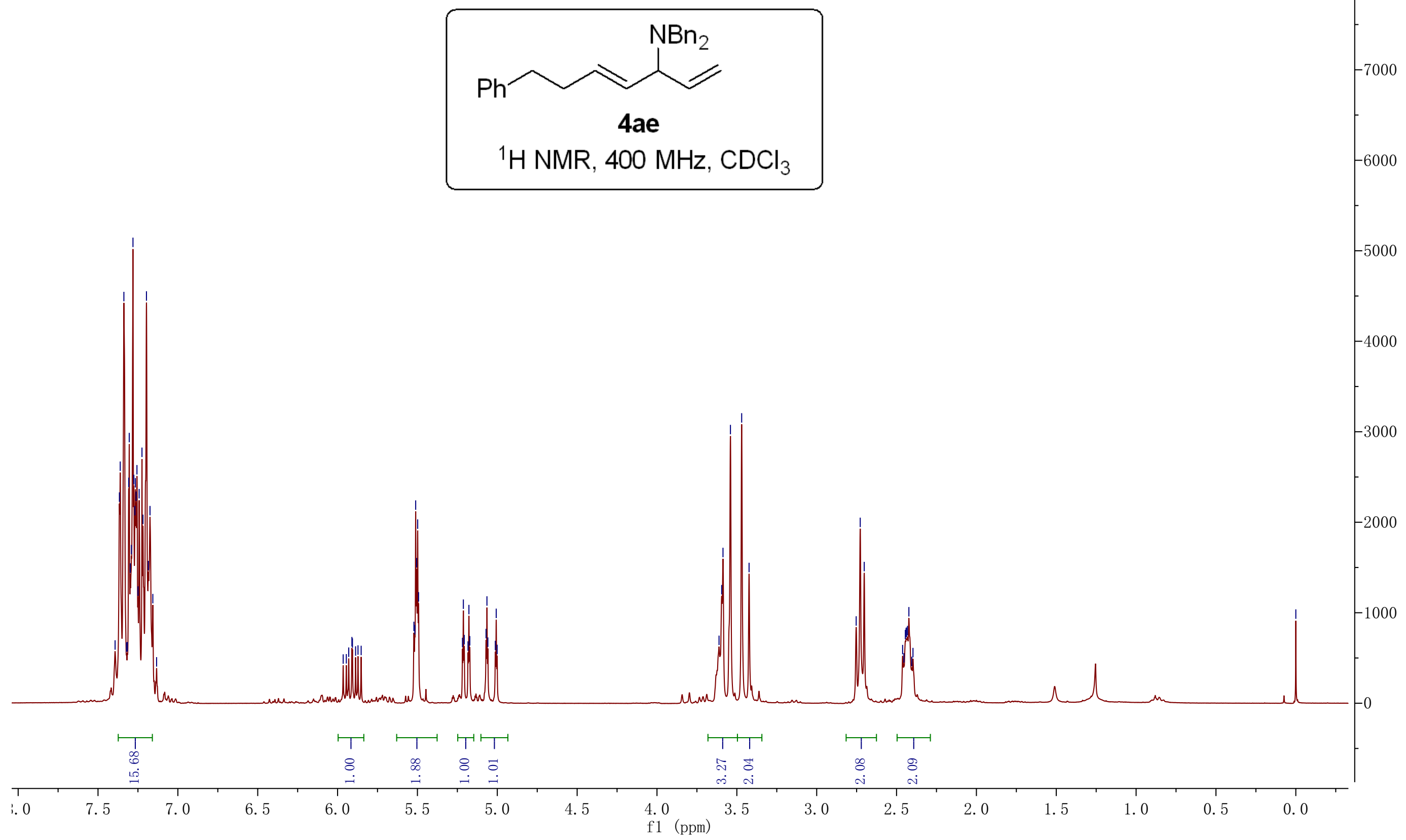


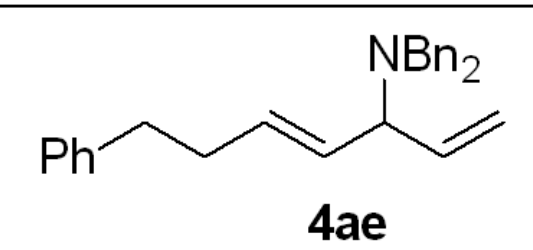

${ }^{1} \mathrm{H} \mathrm{NMR}, 400 \mathrm{MHz}, \mathrm{CDCl}_{3}$ 

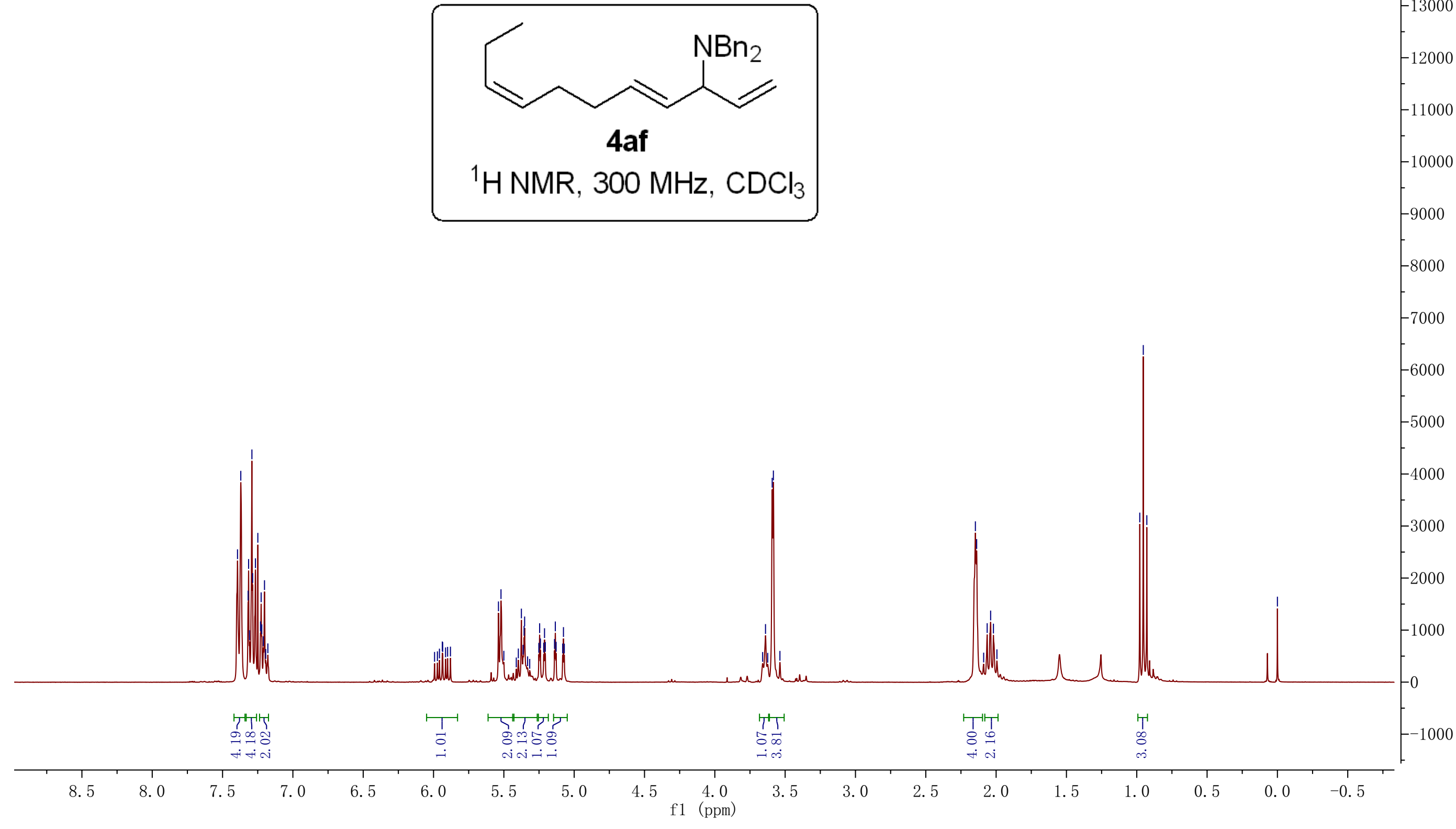


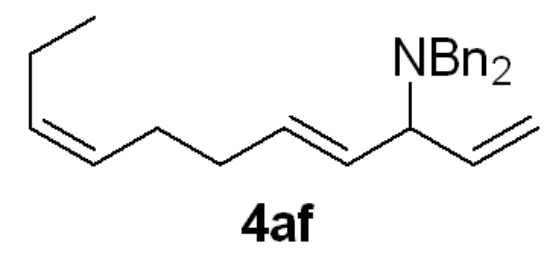

${ }^{13} \mathrm{C} \mathrm{NMR}, 75 \mathrm{MHz}, \mathrm{CDCl}_{3}$

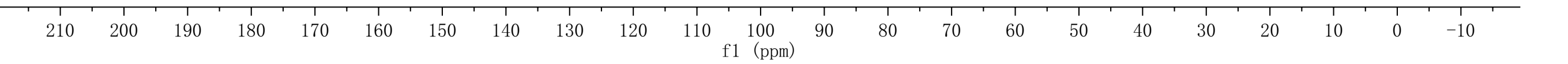




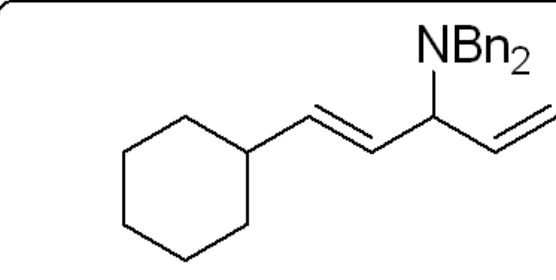

4ag

${ }^{1} \mathrm{H} \mathrm{NMR}, 400 \mathrm{MHz}, \mathrm{CDCl}_{3}$

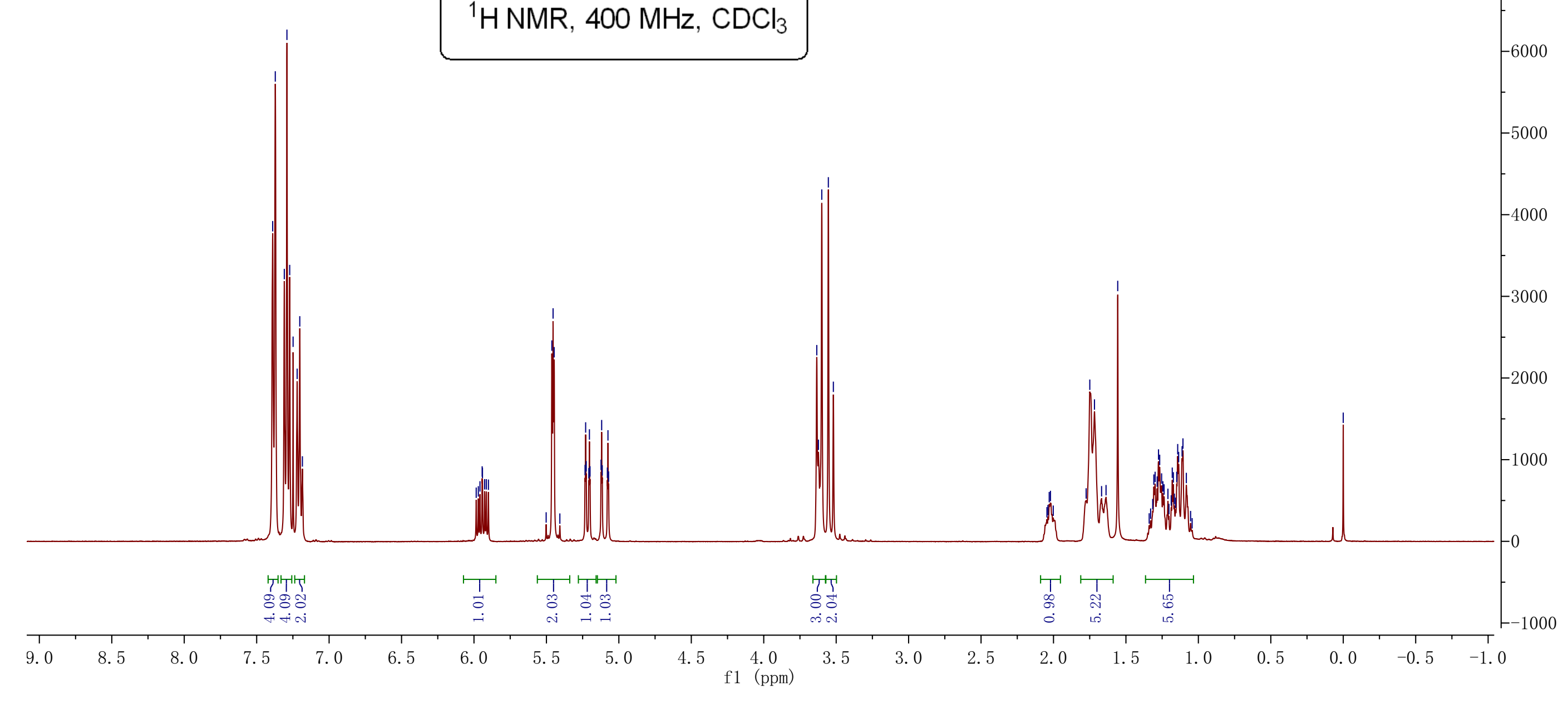




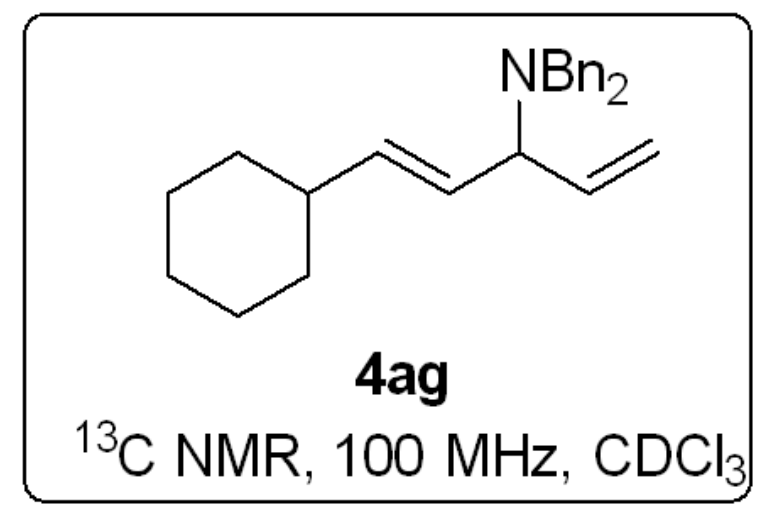



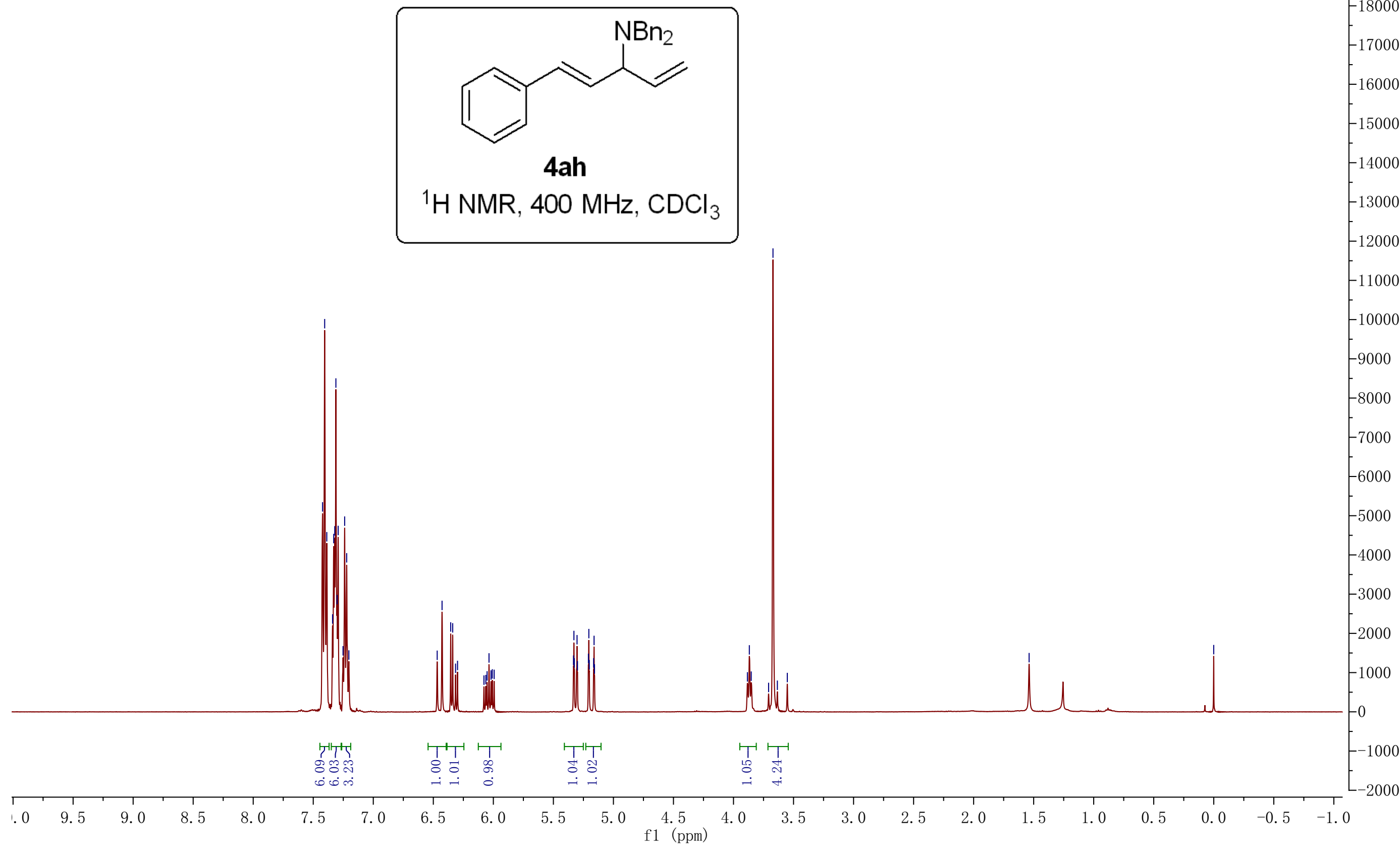


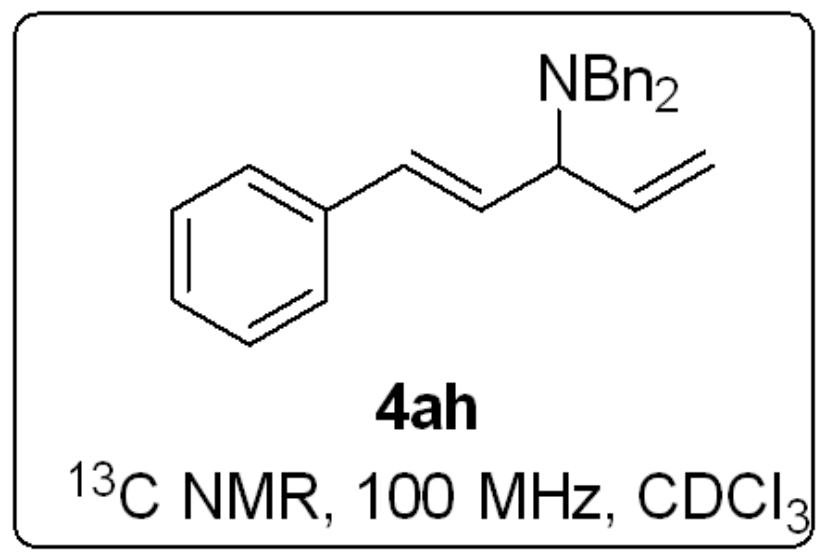



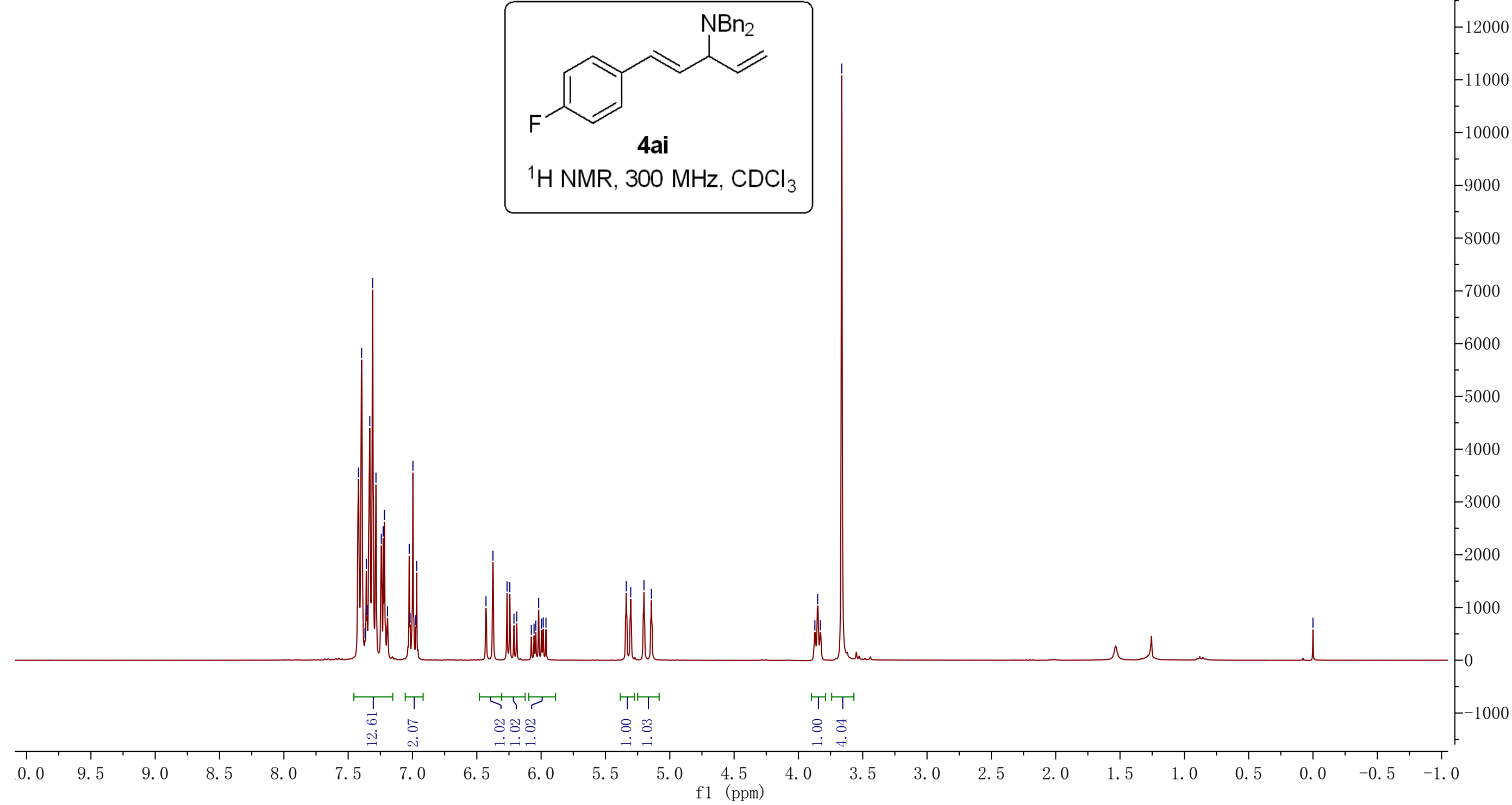


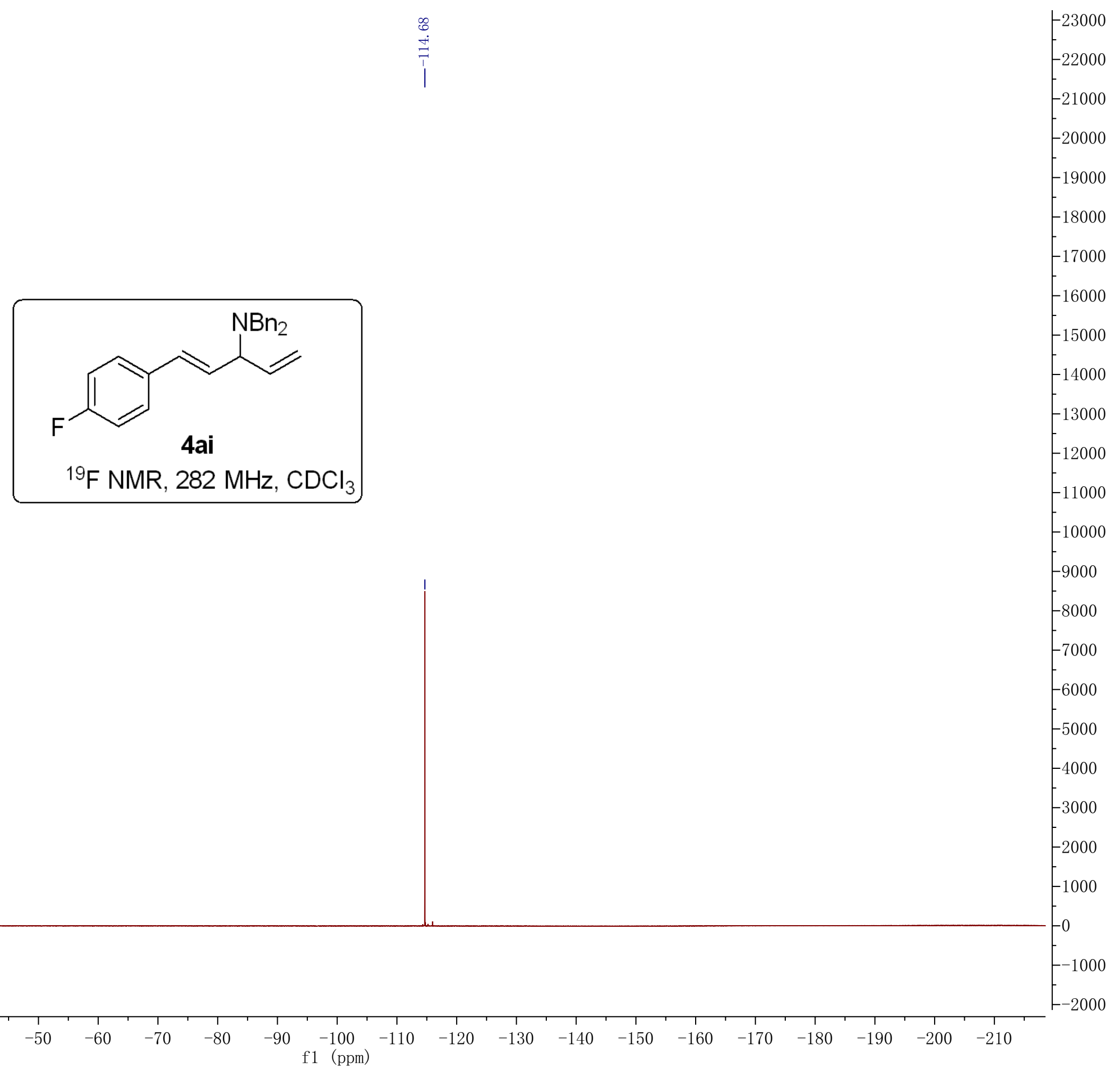




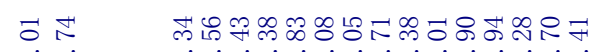

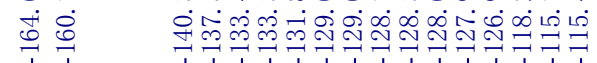 \\ $\vec{\imath}$ रे}

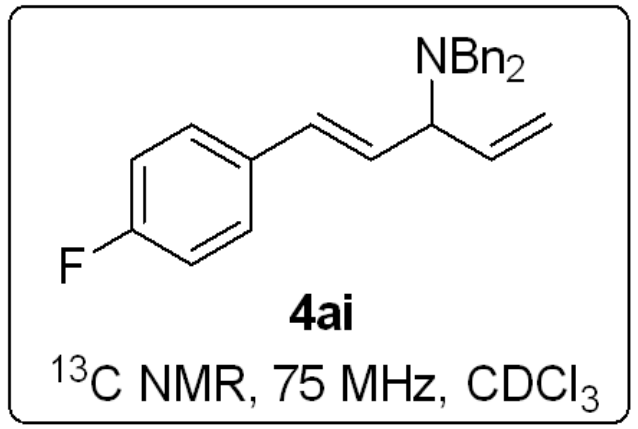



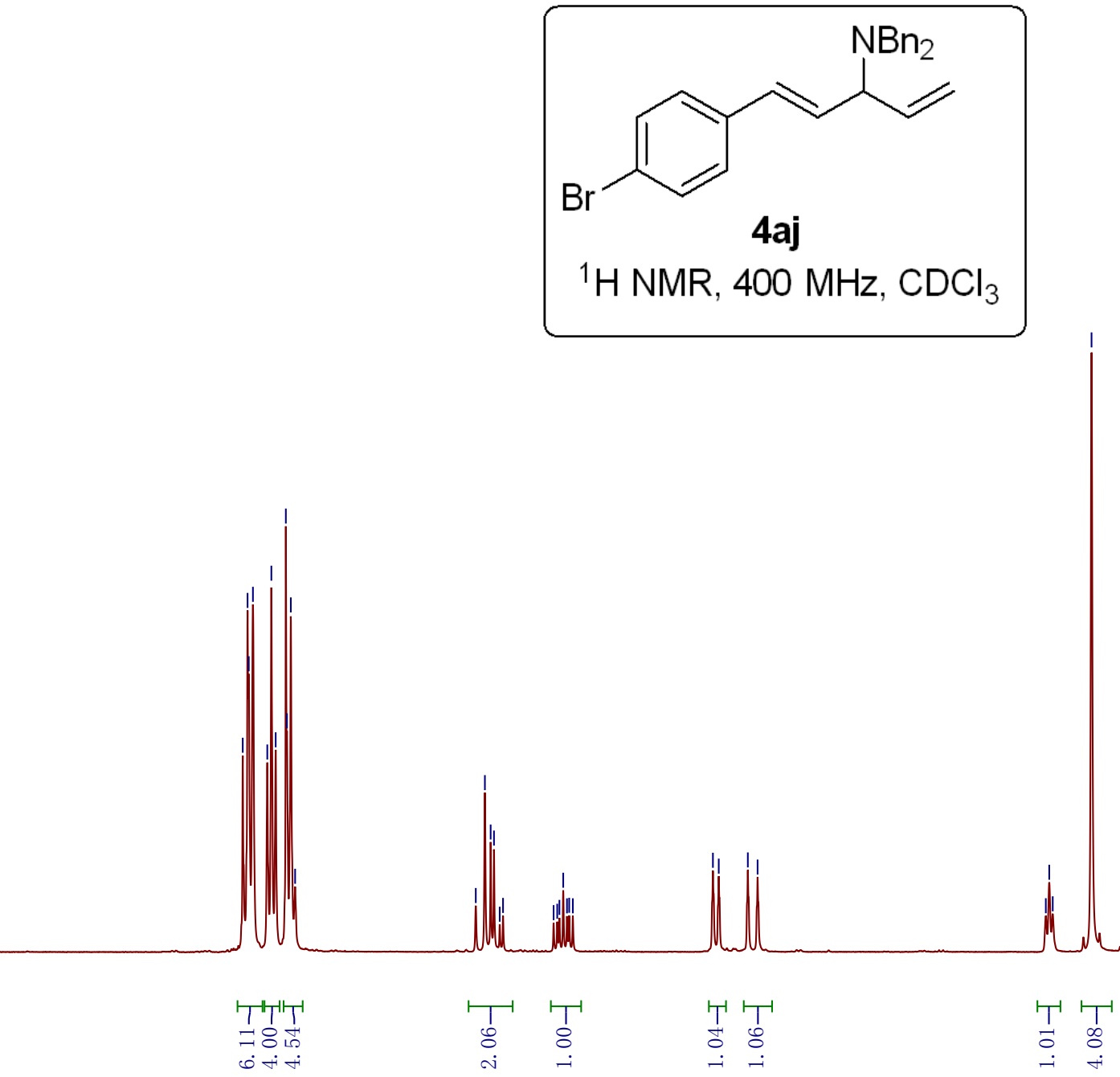

$\stackrel{\infty}{\circ}$ 


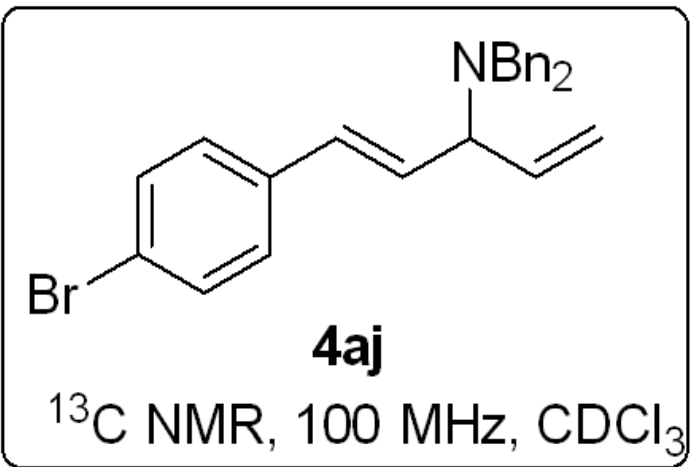



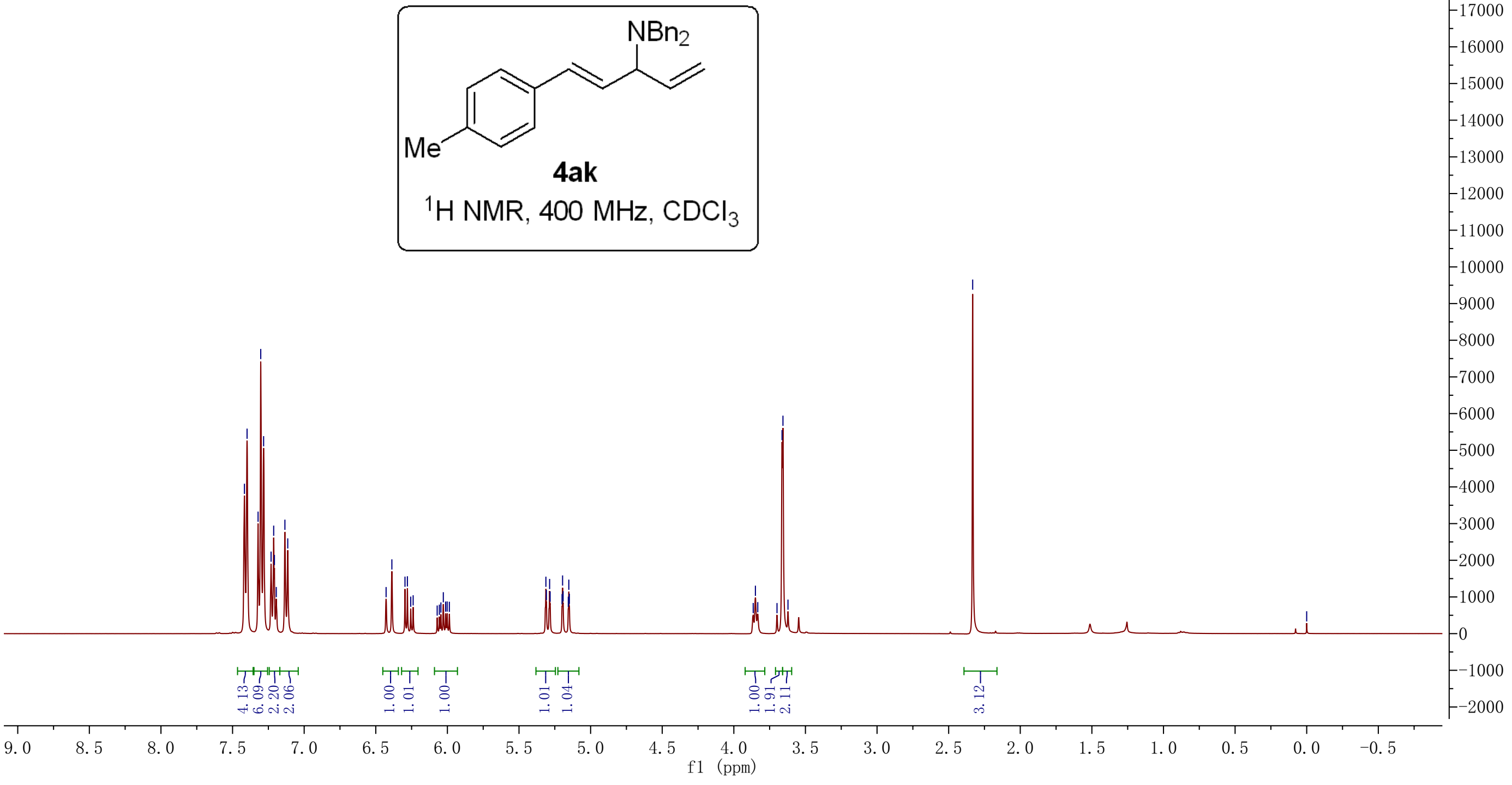


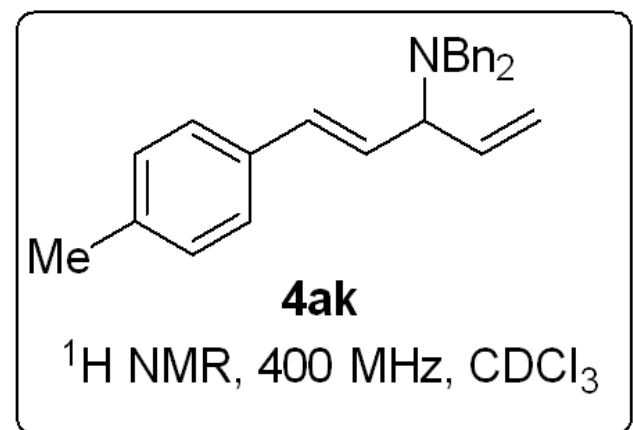




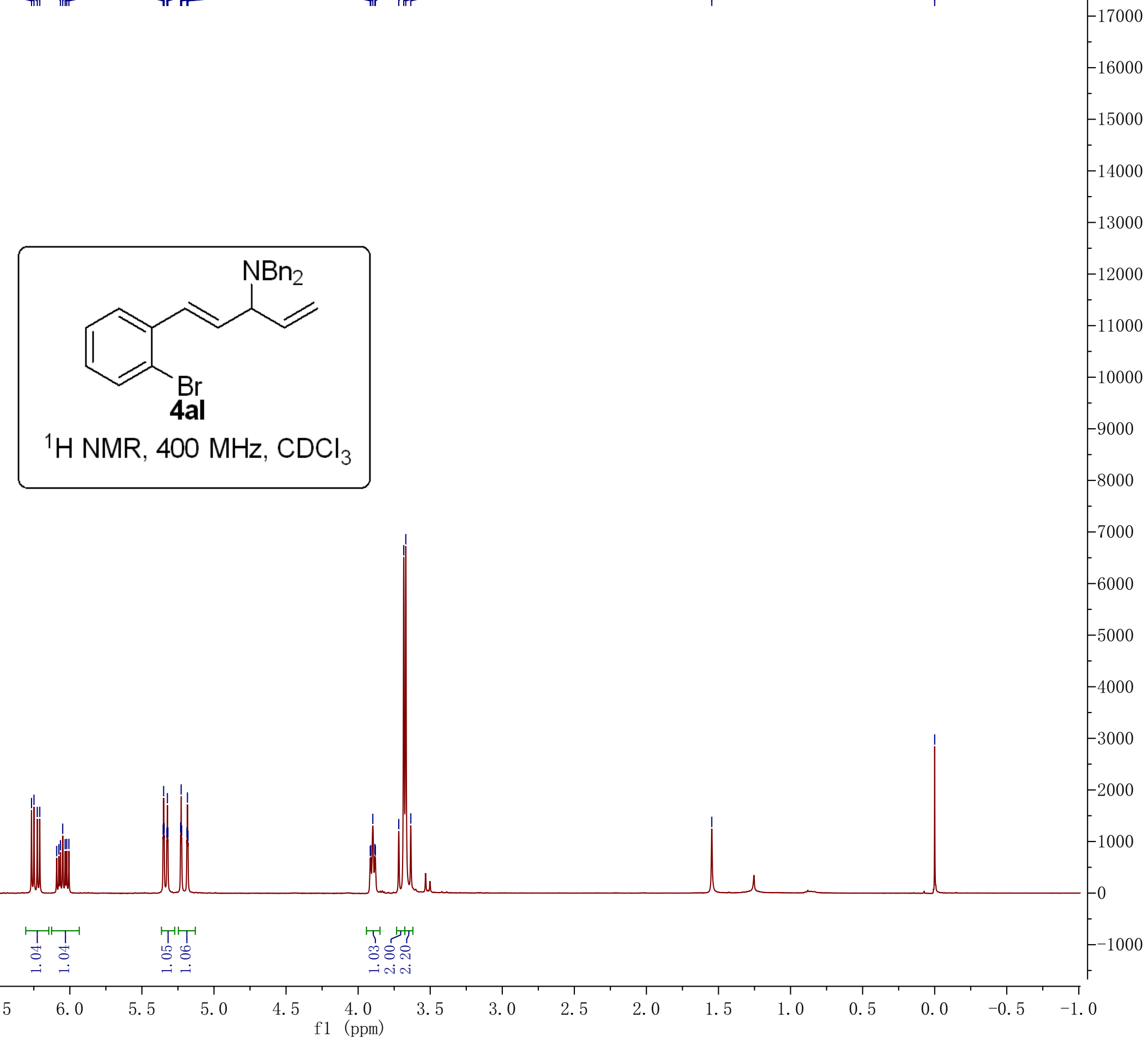


<smiles>C=CC(C)/C=C/c1ccccc1Br</smiles> 

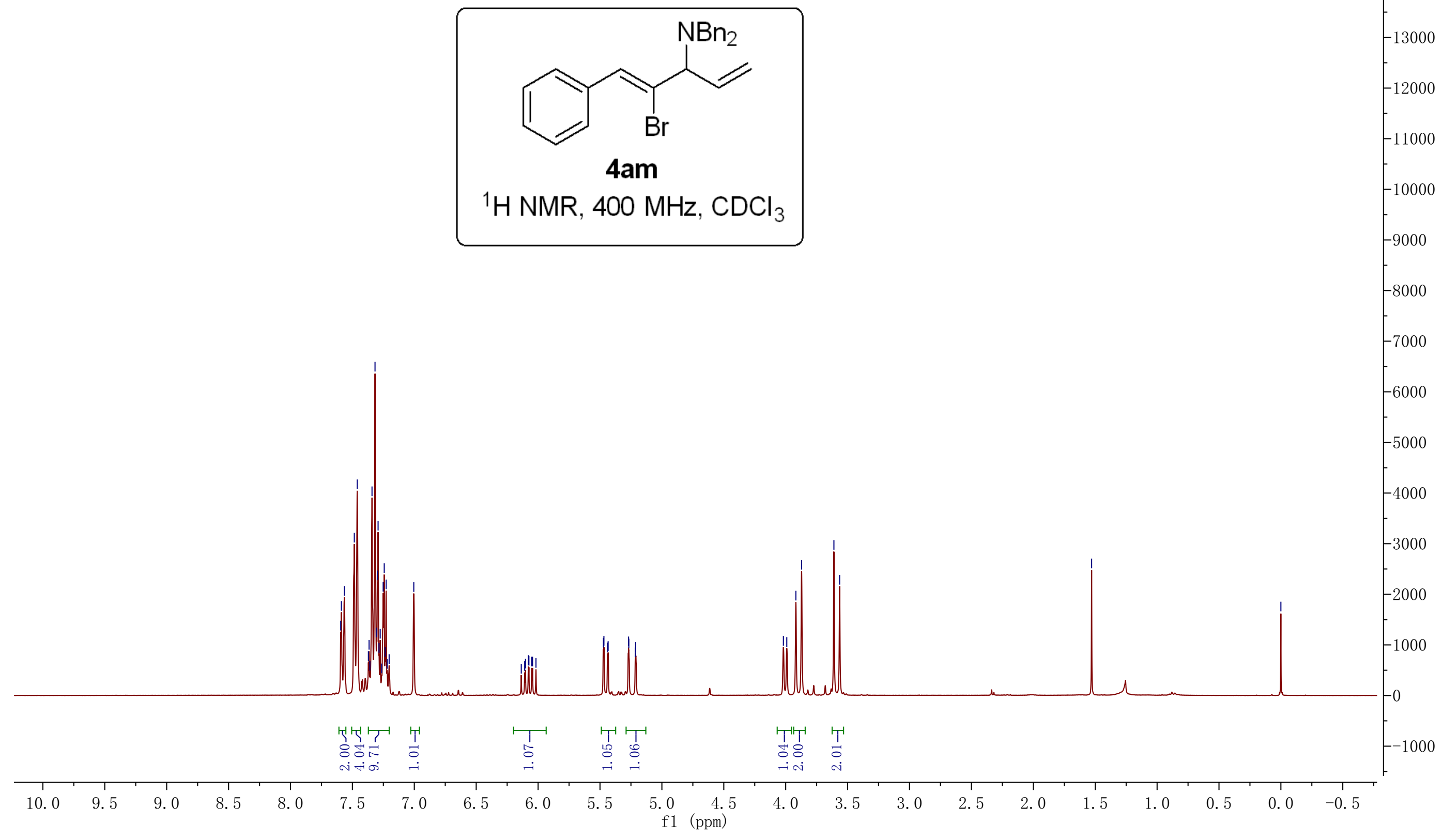


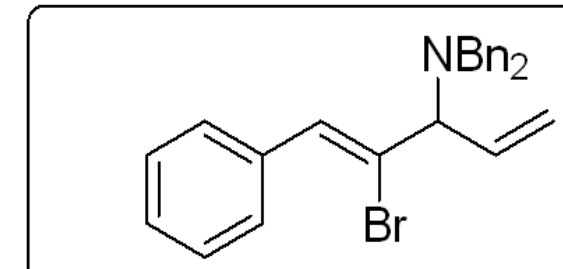

4am

${ }^{13} \mathrm{C}$ NMR, $100 \mathrm{MHz}, \mathrm{CDCl}_{3}$ 

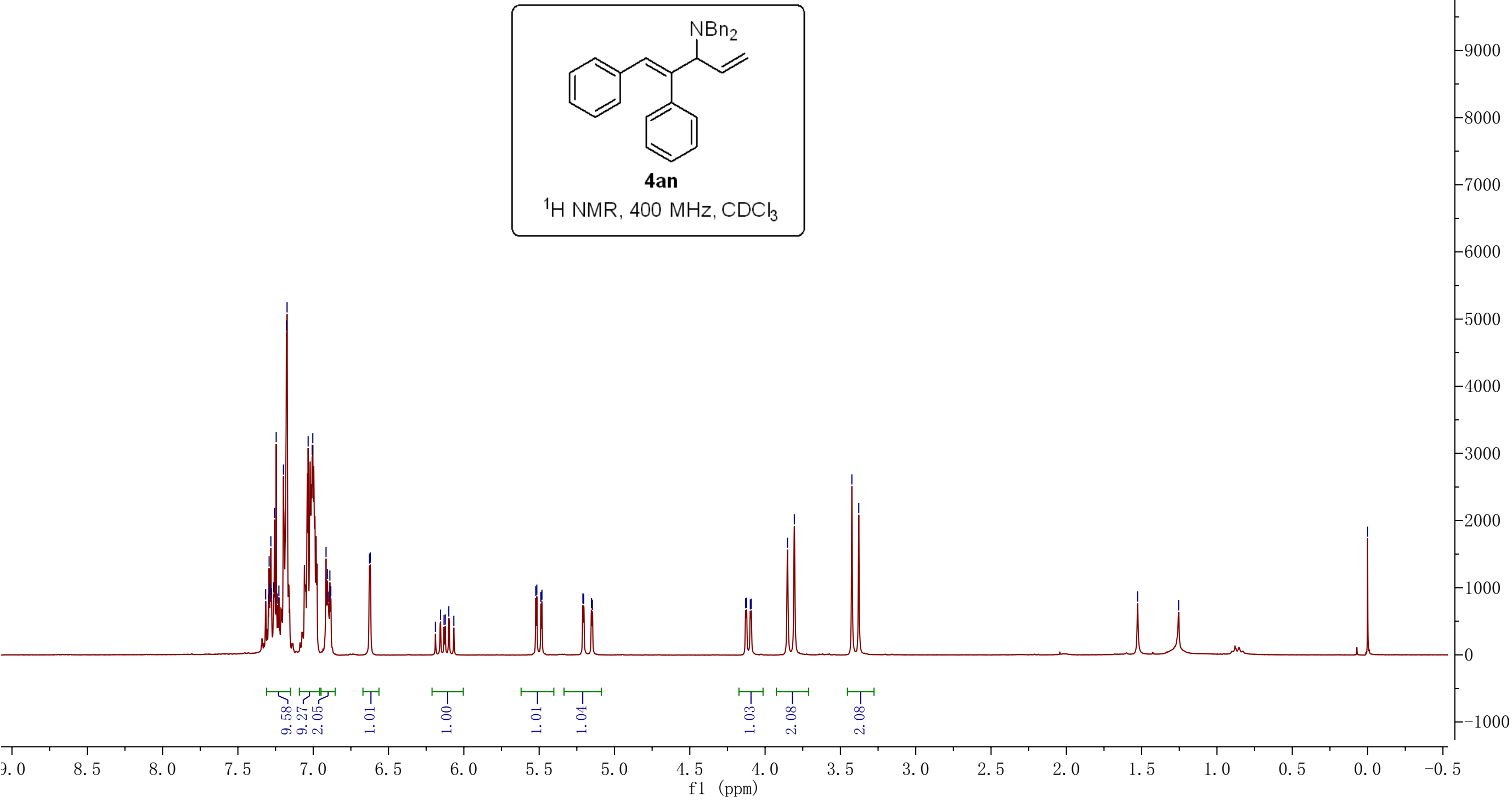


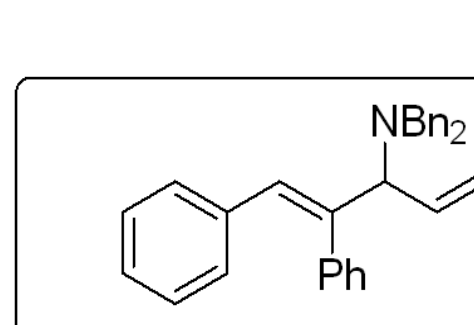

4an

${ }^{13} \mathrm{C} \mathrm{NMR}, 100 \mathrm{MHz}, \mathrm{CDCl}_{3}$ 

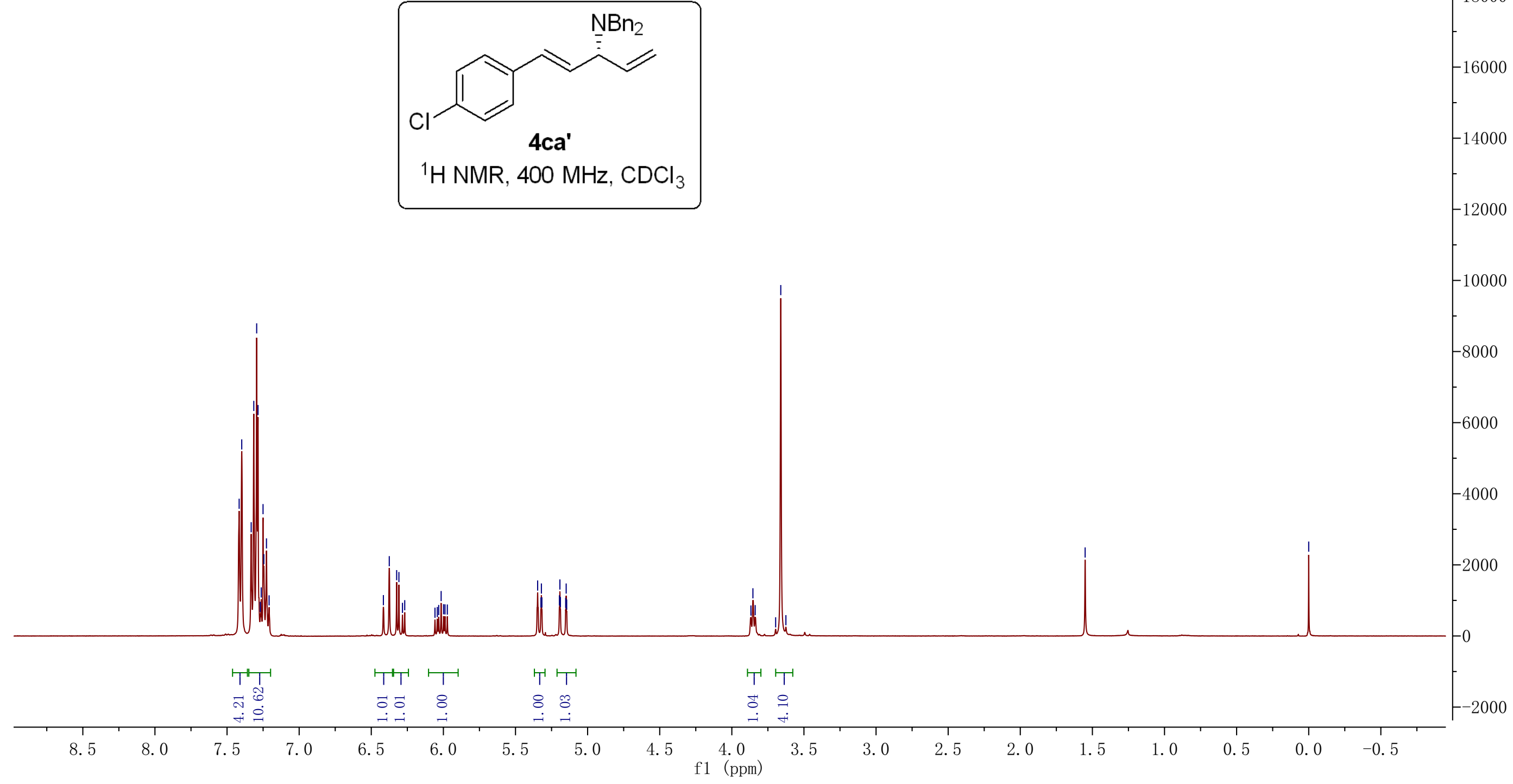


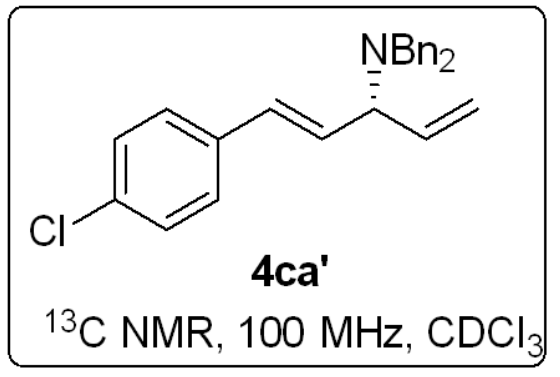



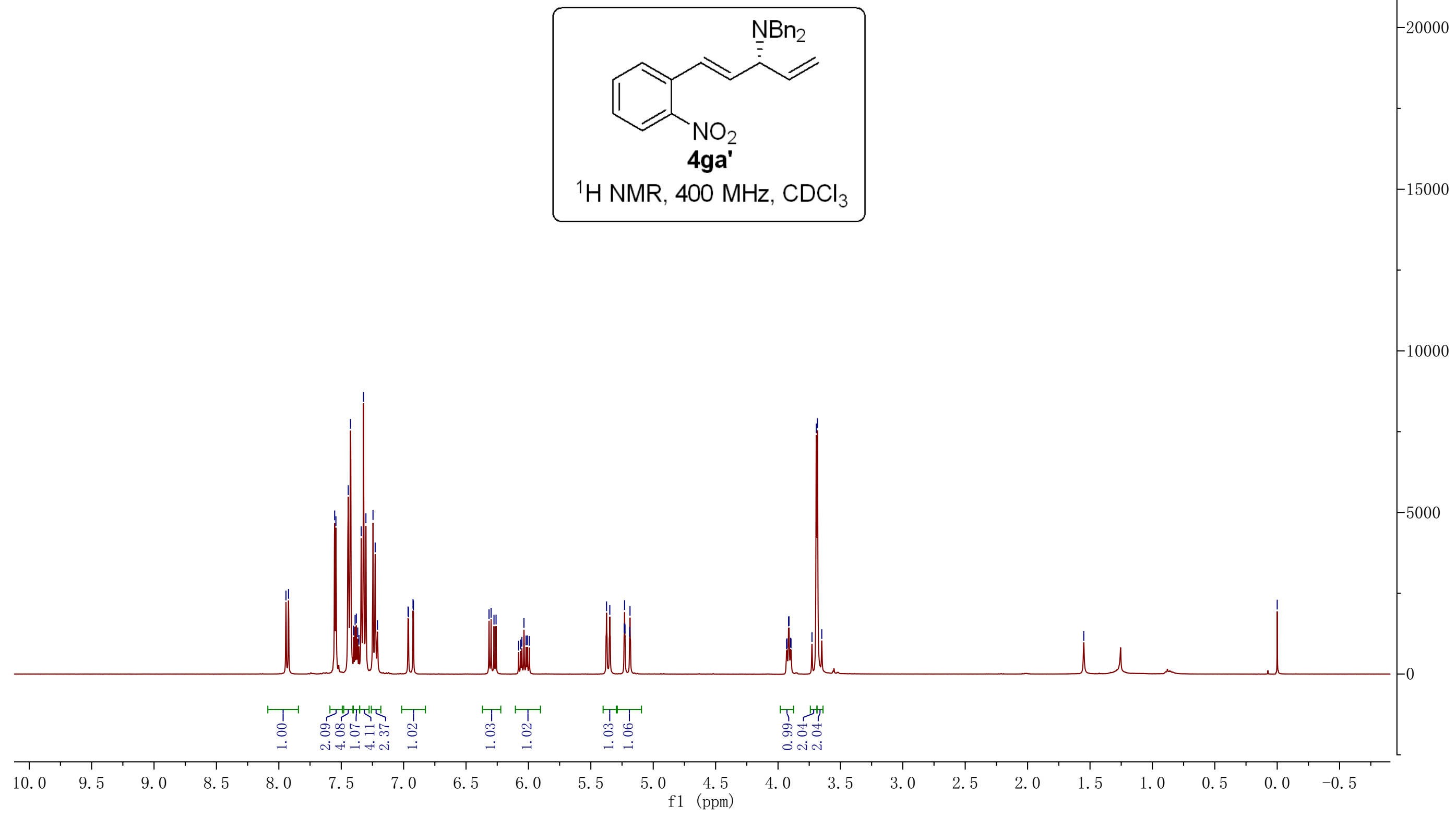

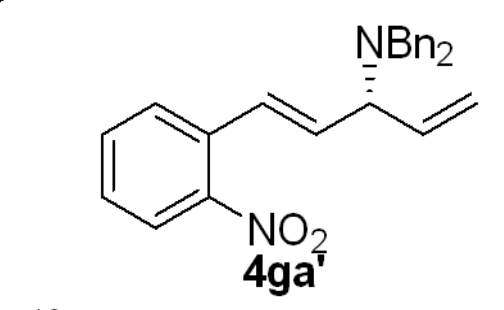

${ }^{13} \mathrm{C} \mathrm{NMR}, 100 \mathrm{MHz}, \mathrm{CDCl}_{3}$ 


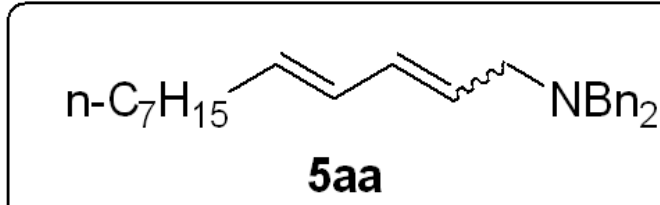

$E / Z=16.7 / 1$

${ }^{1} \mathrm{H} \mathrm{NMR}, 400 \mathrm{MHz}, \mathrm{CDCl}_{3}$
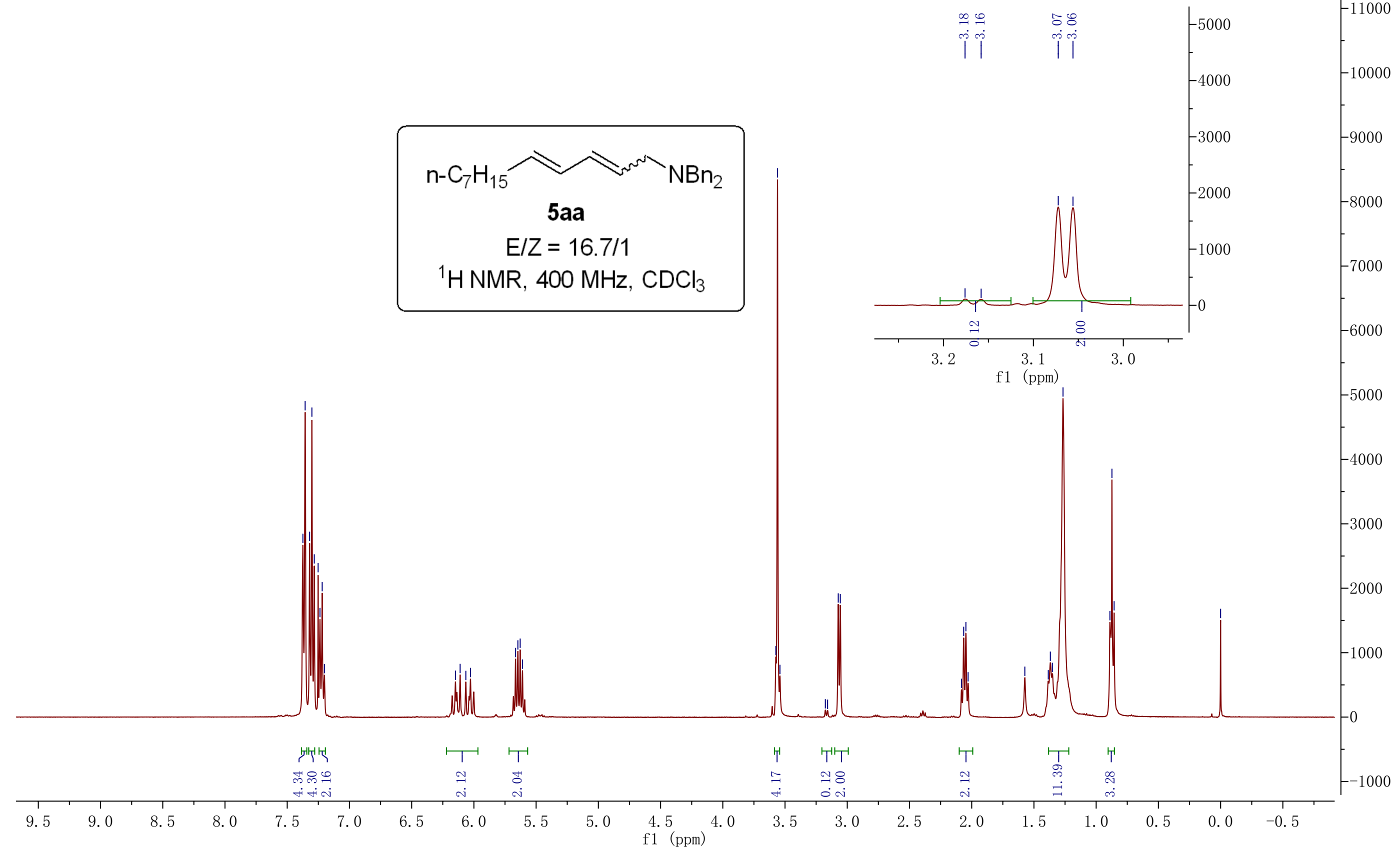


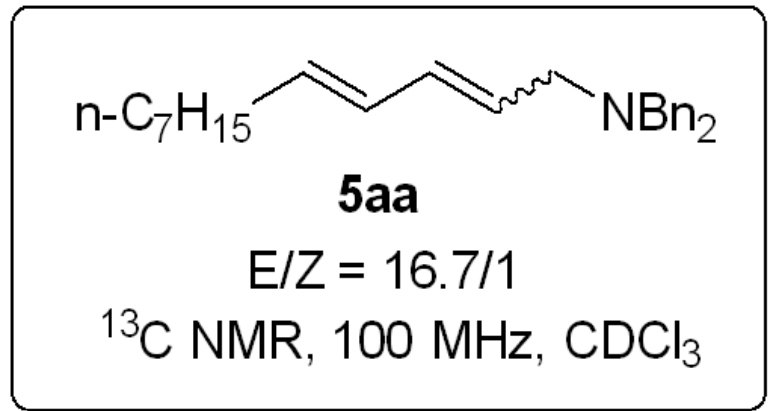




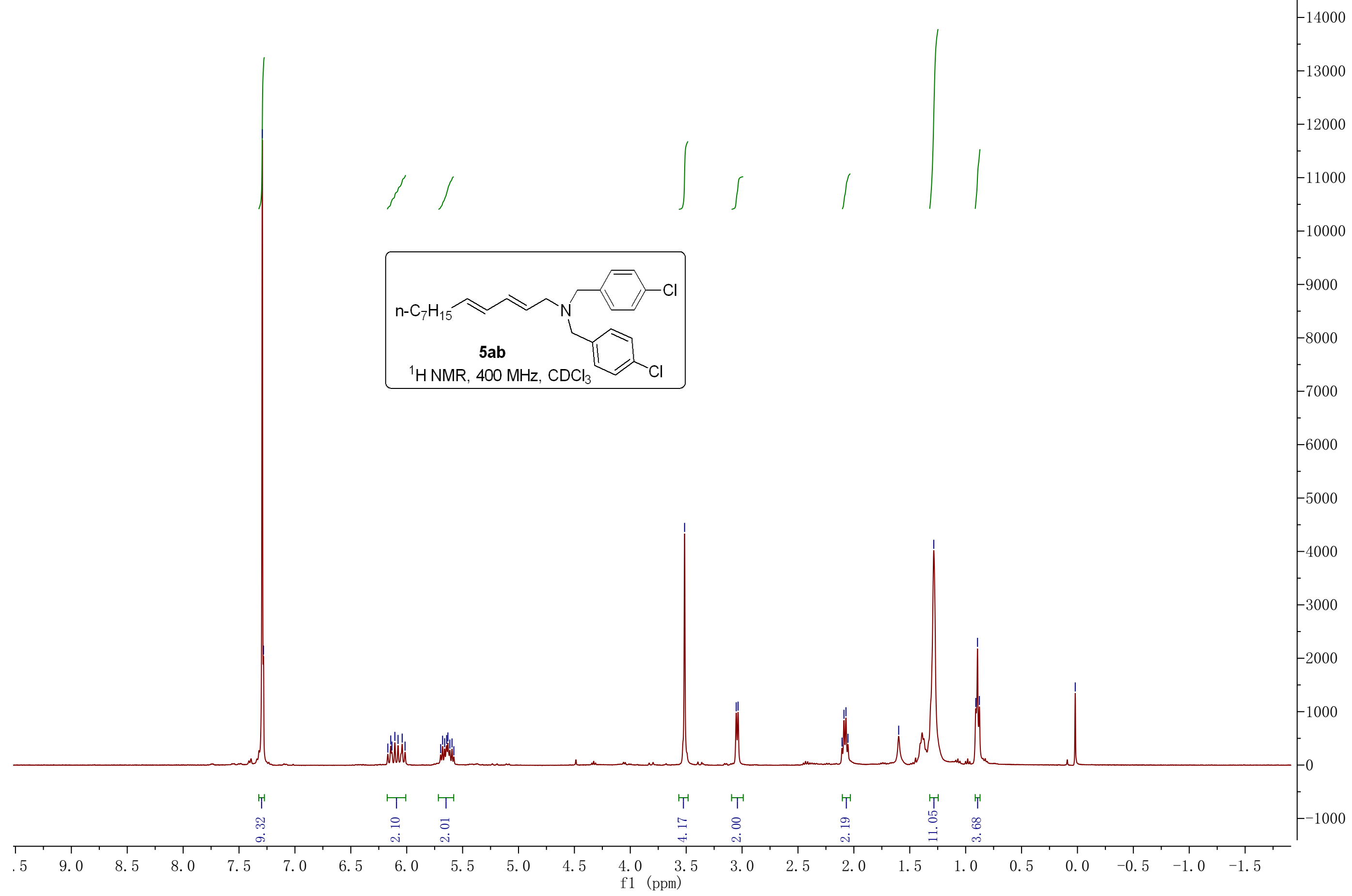




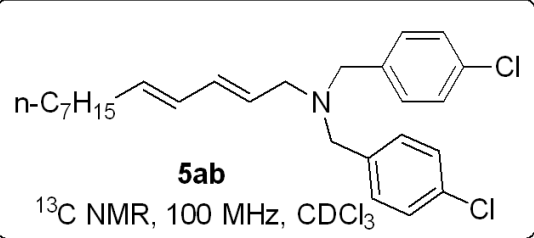




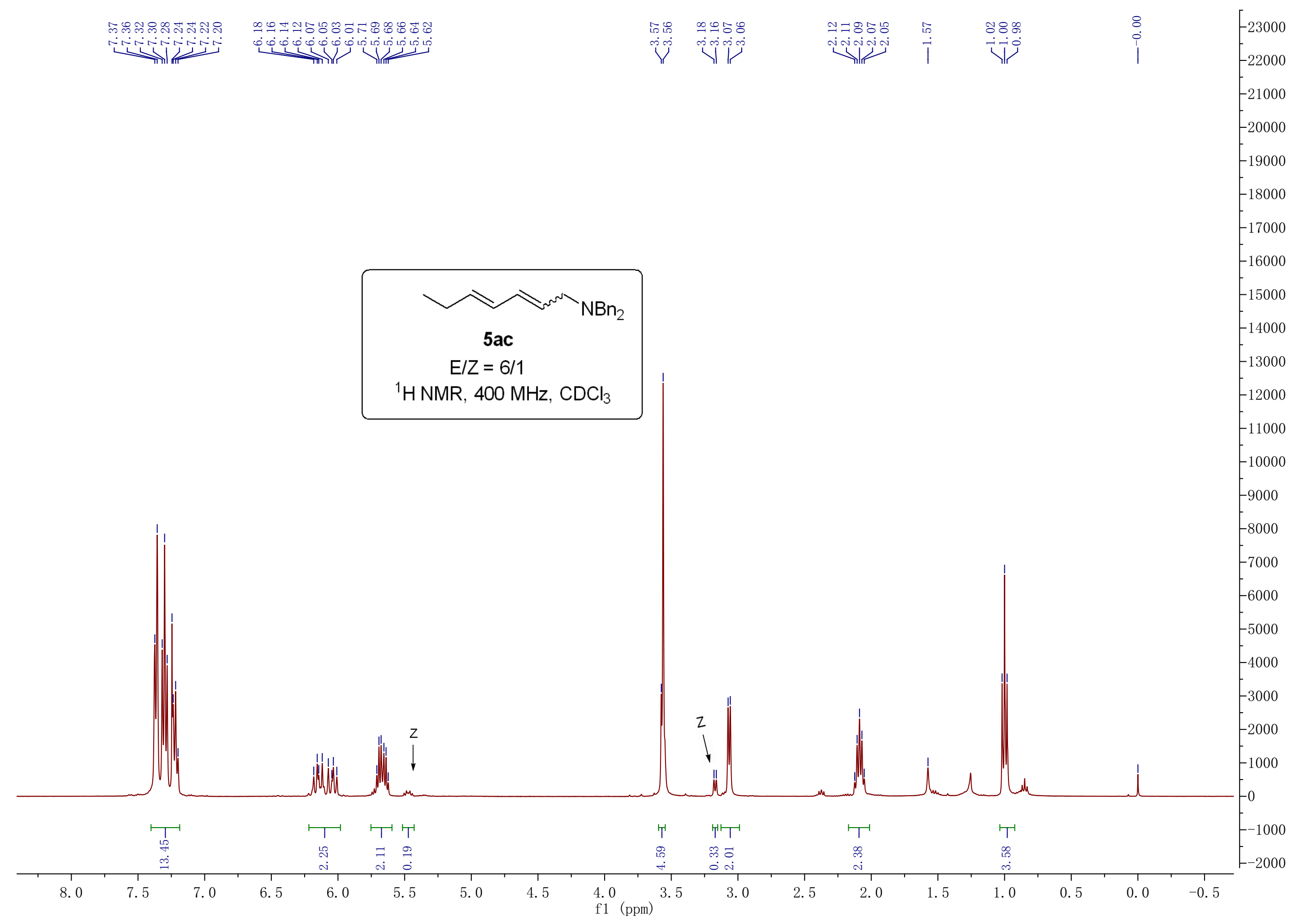




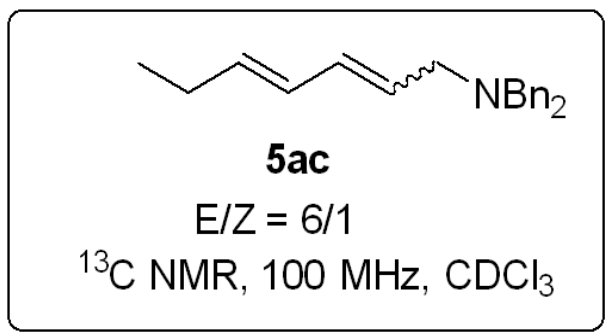




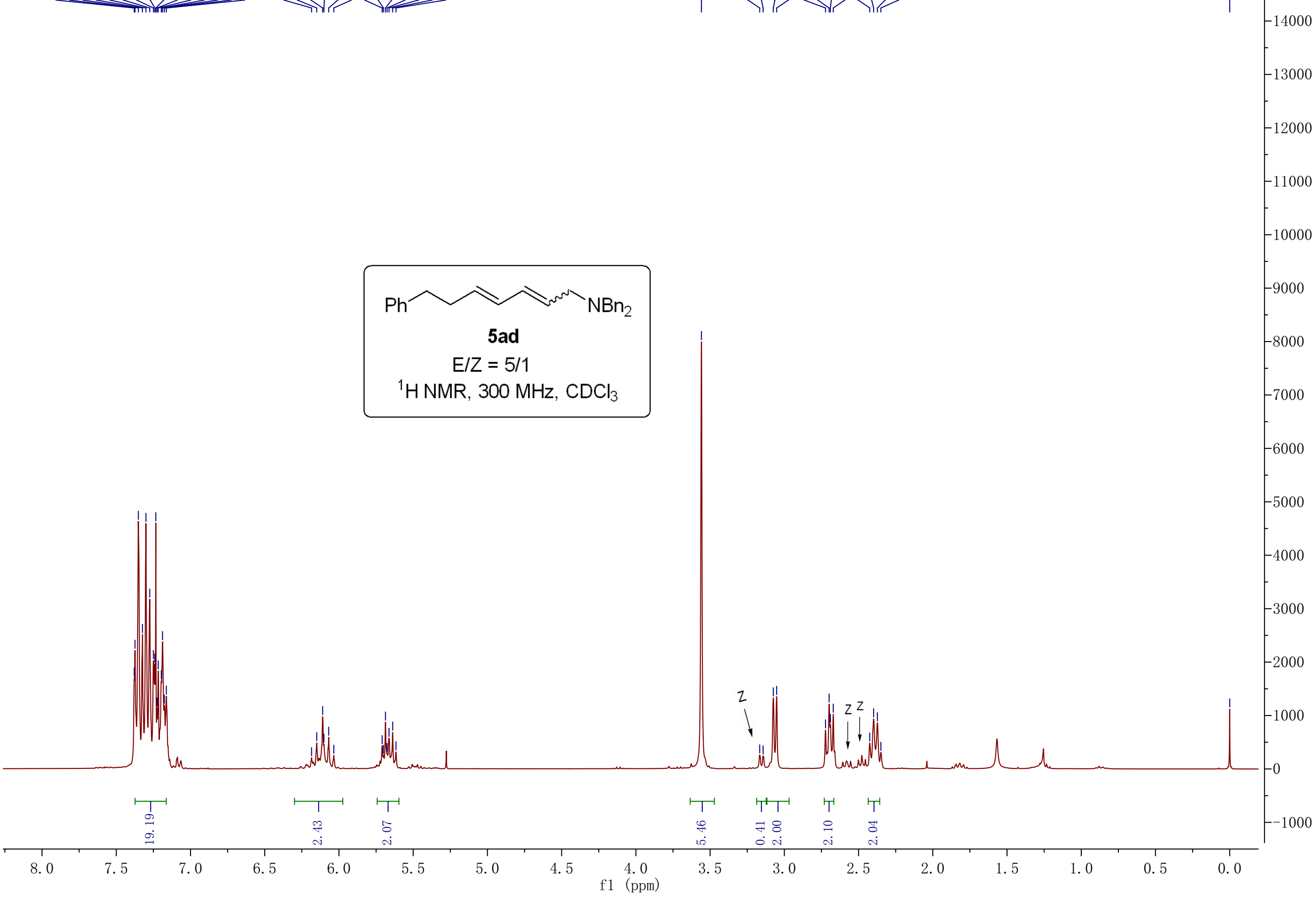




$$
\begin{gathered}
5 \text { ad } \\
E / Z=5 / 1 \\
{ }^{13} \mathrm{C} \mathrm{NMR}, 75 \mathrm{MHz}, \mathrm{CDCl}_{3}
\end{gathered}
$$

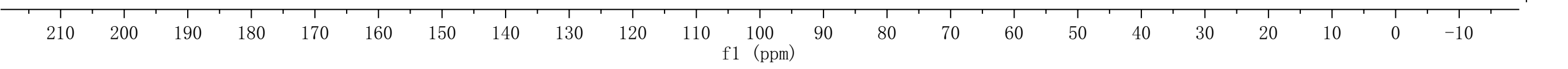




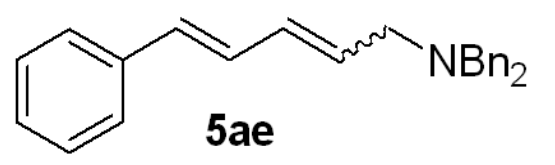

$E / Z=11.8 / 1$

${ }^{1} \mathrm{HNMR}, 400 \mathrm{MHz}, \mathrm{CDCl}_{3}$

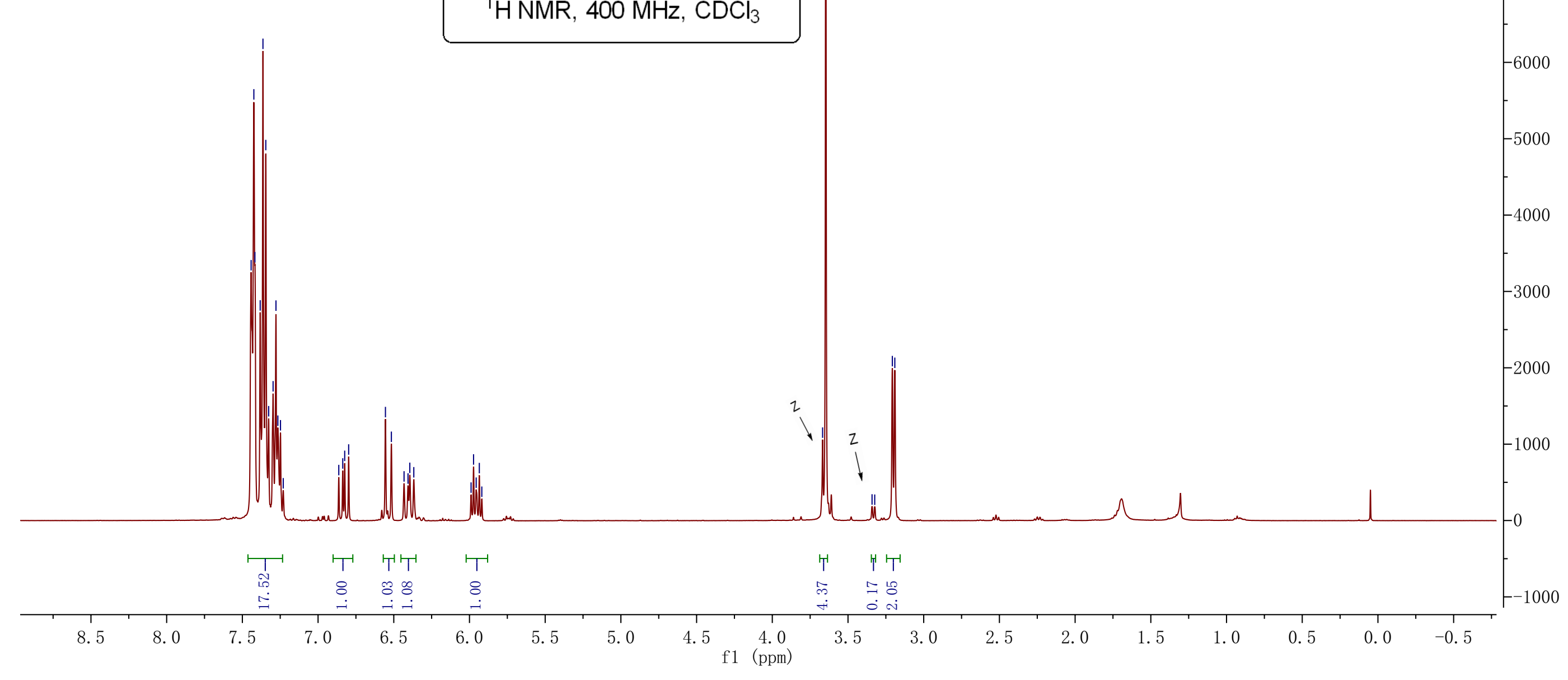




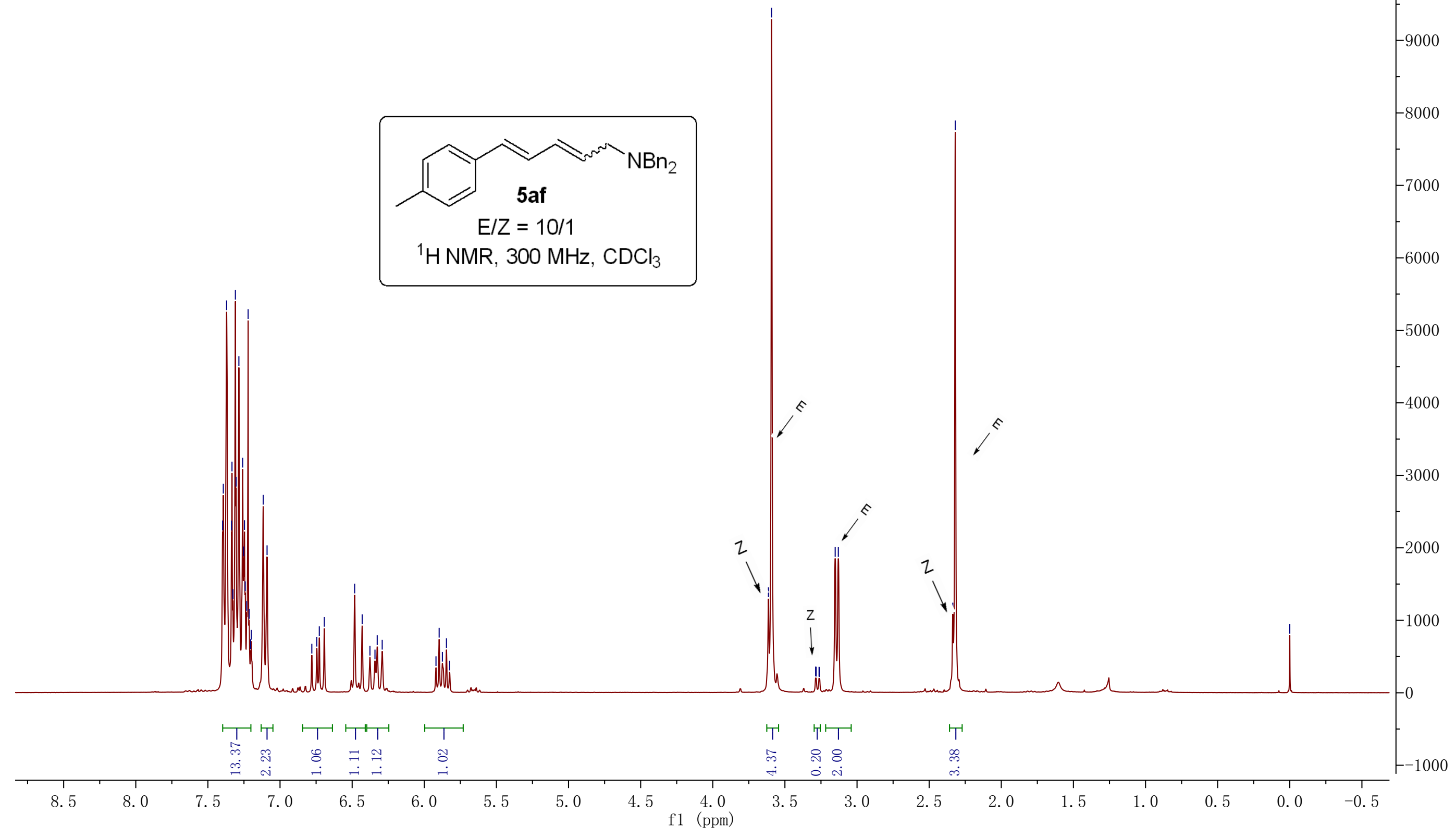




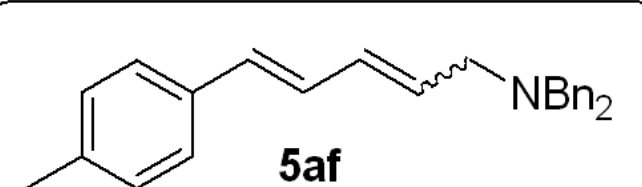

$E / Z=10 / 1$

${ }^{13} \mathrm{C} \mathrm{NMR}, 75 \mathrm{MHz}, \mathrm{CDCl}_{3}$ 

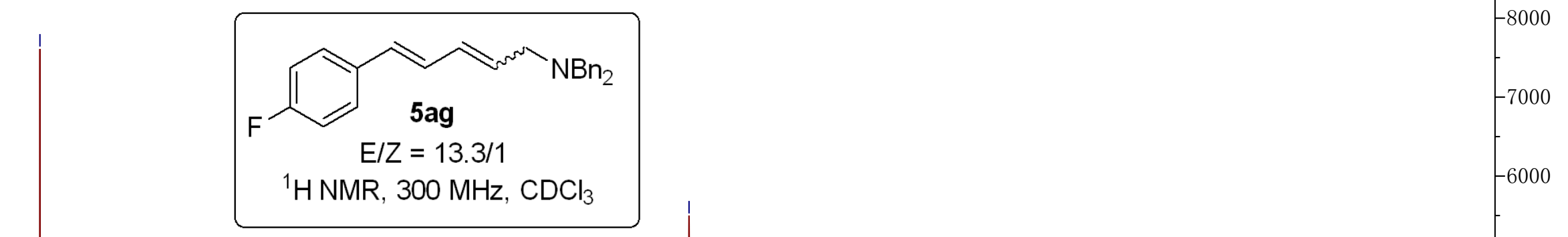

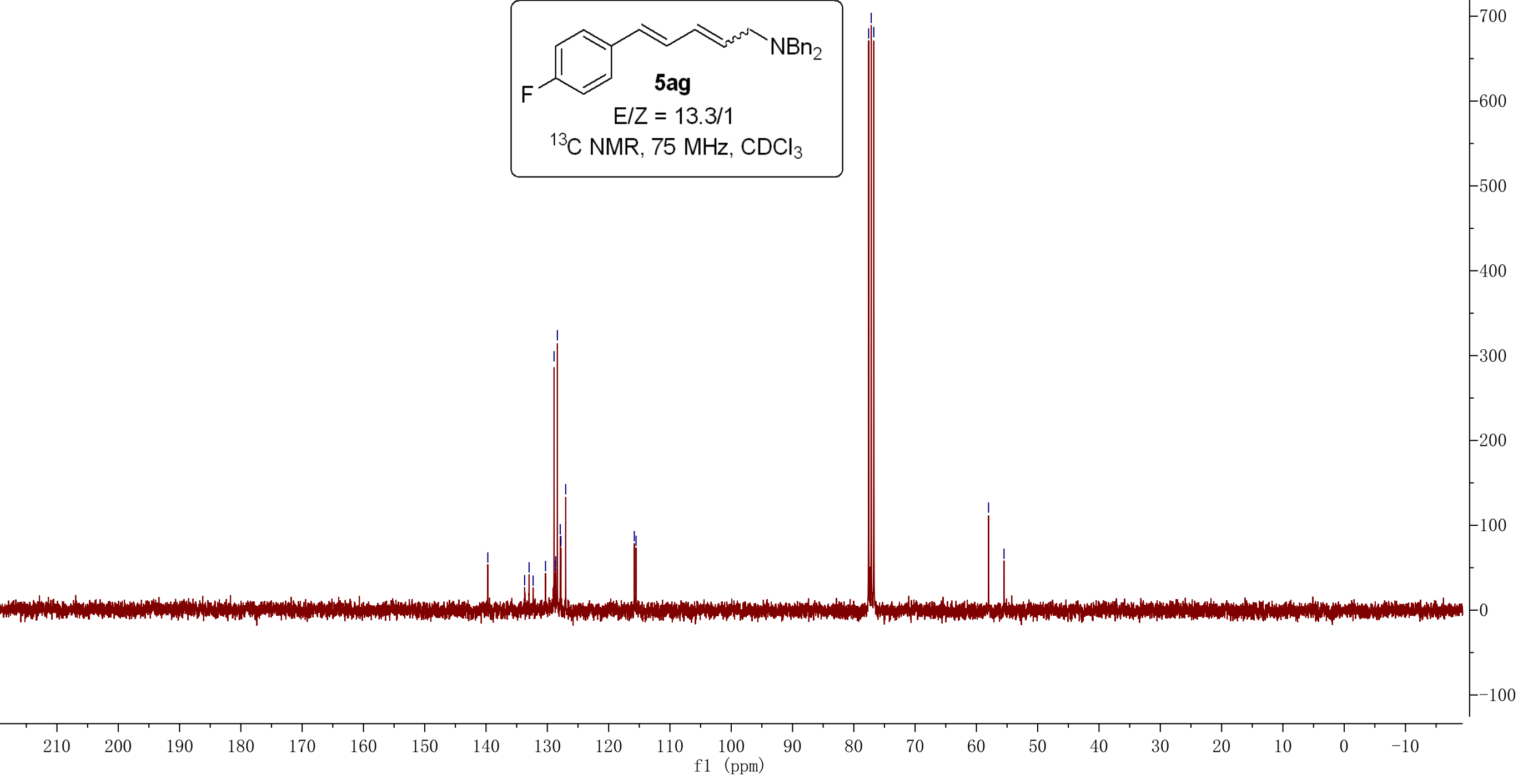

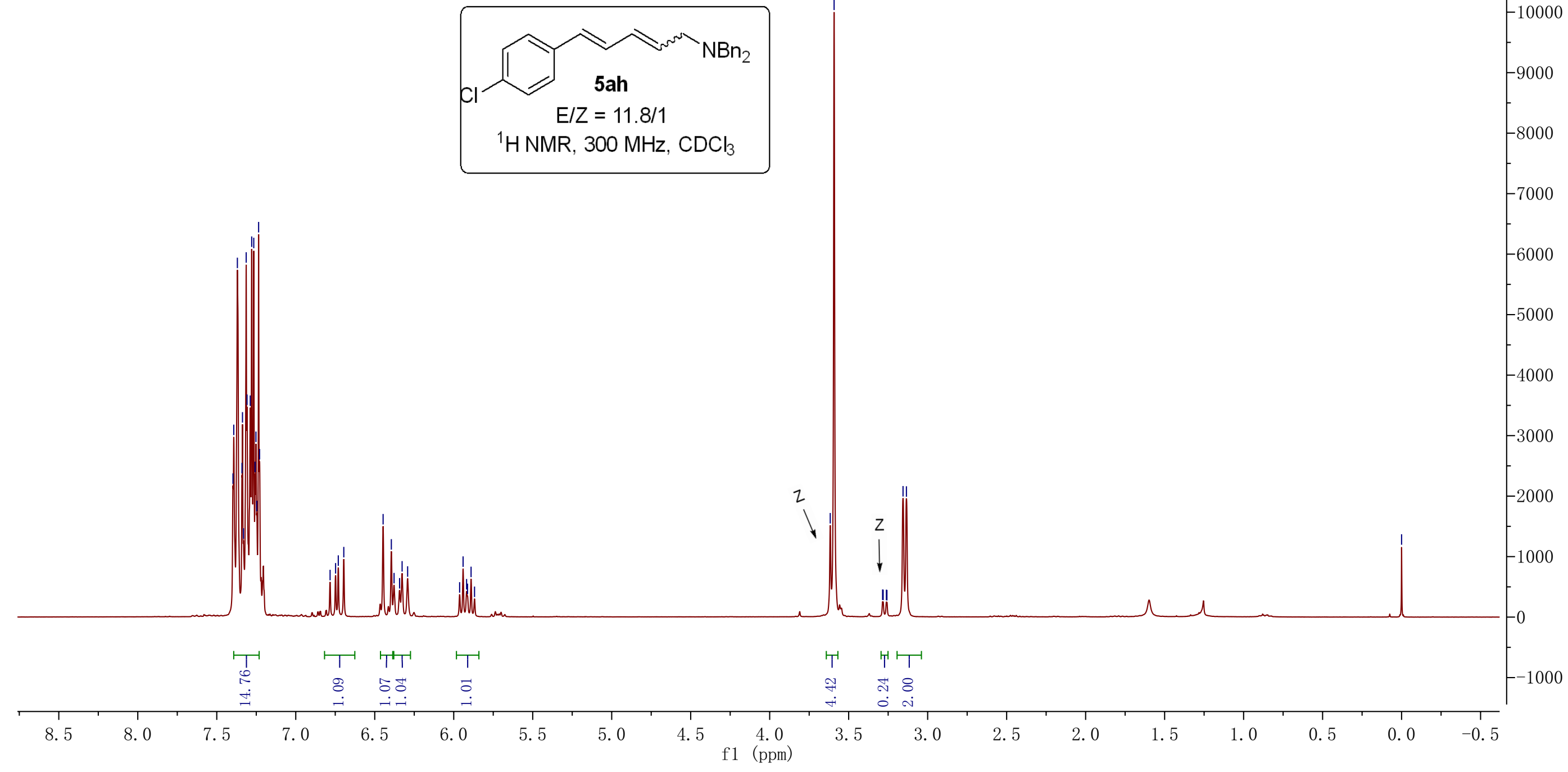


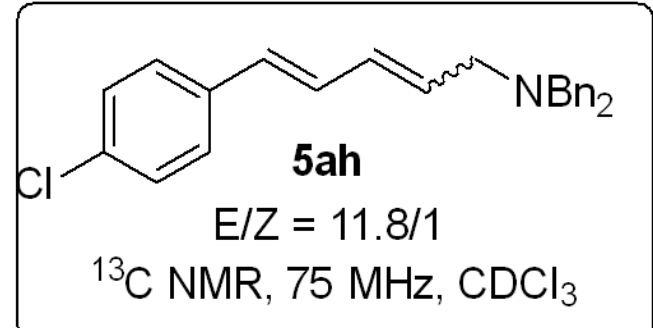



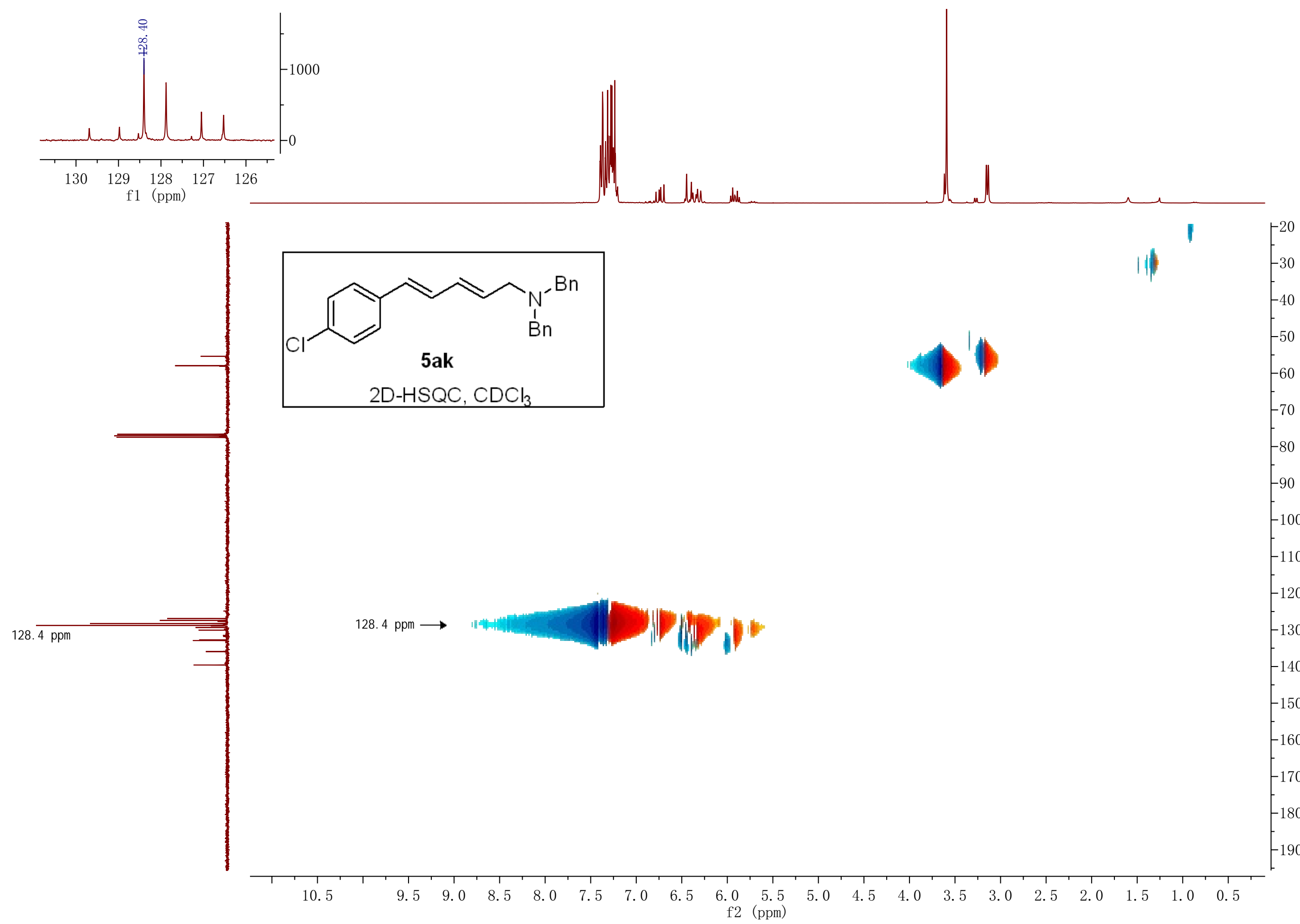

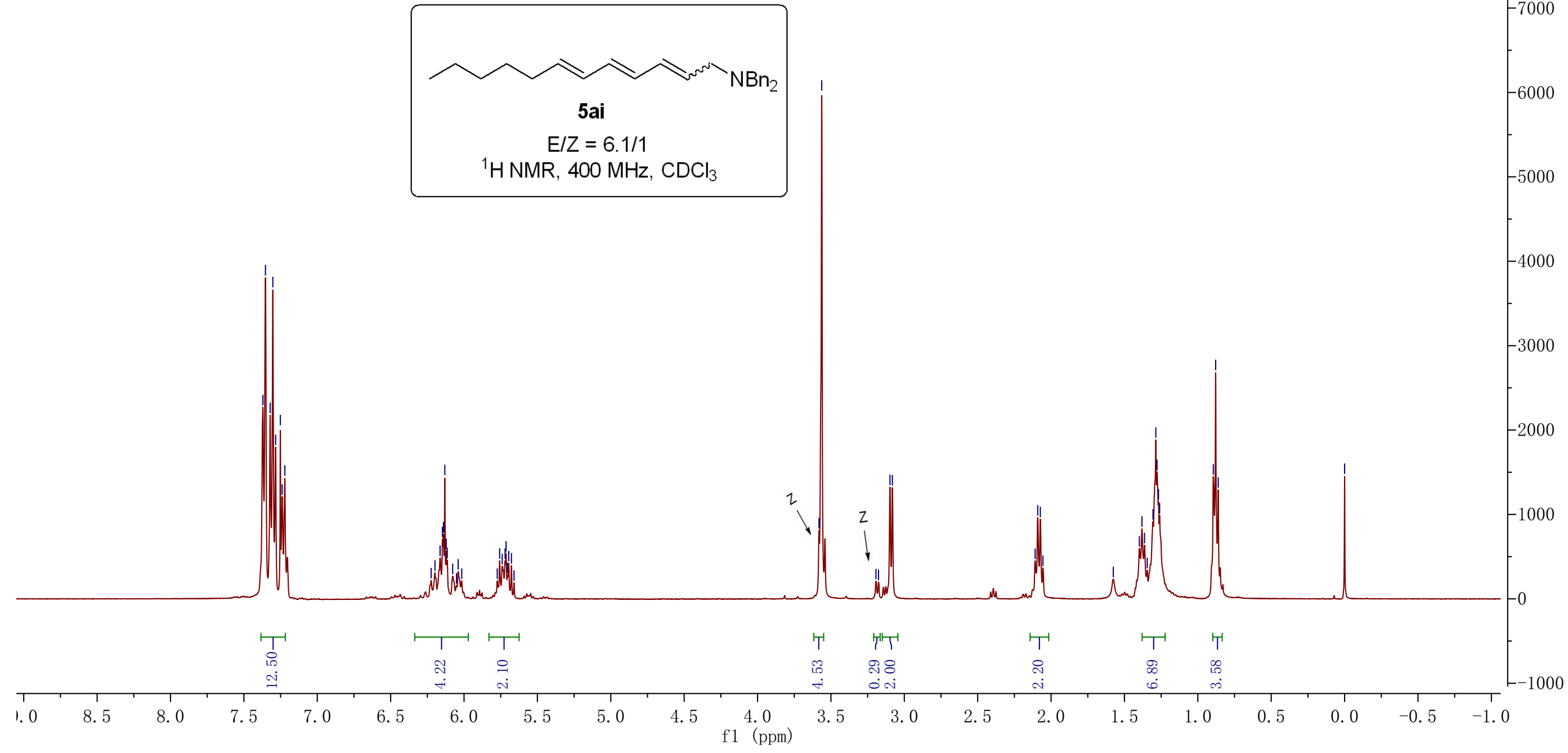


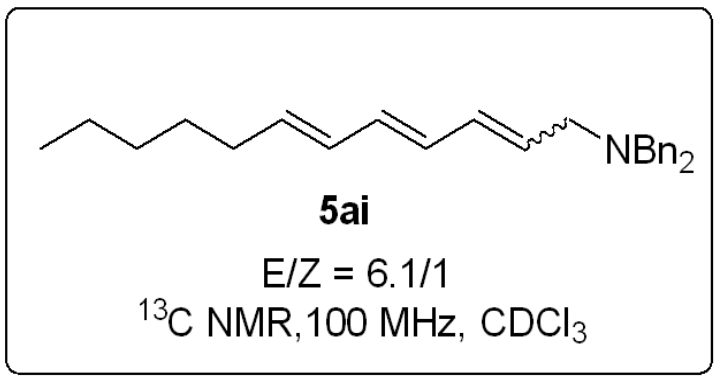

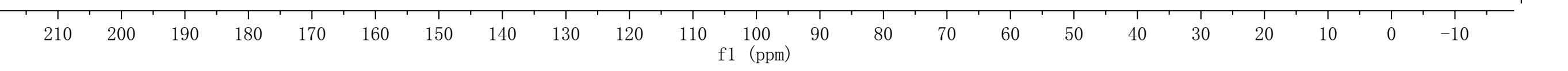



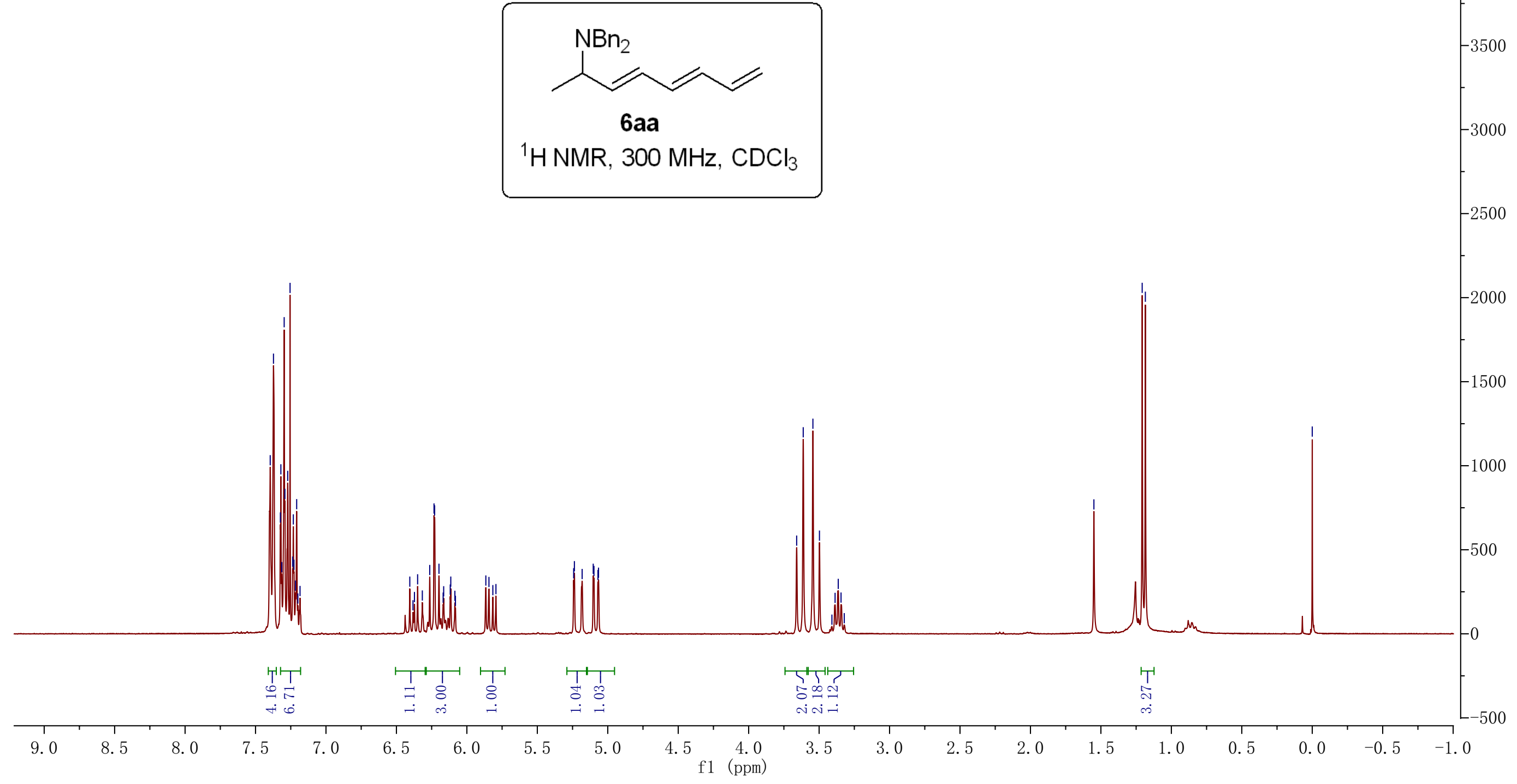


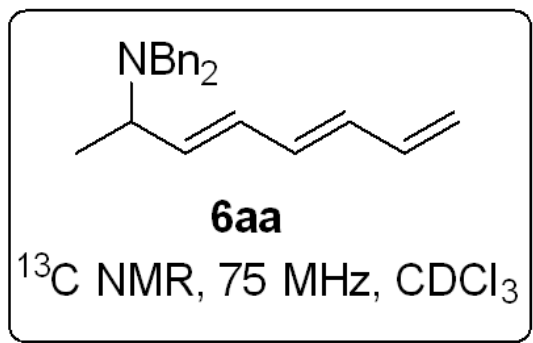




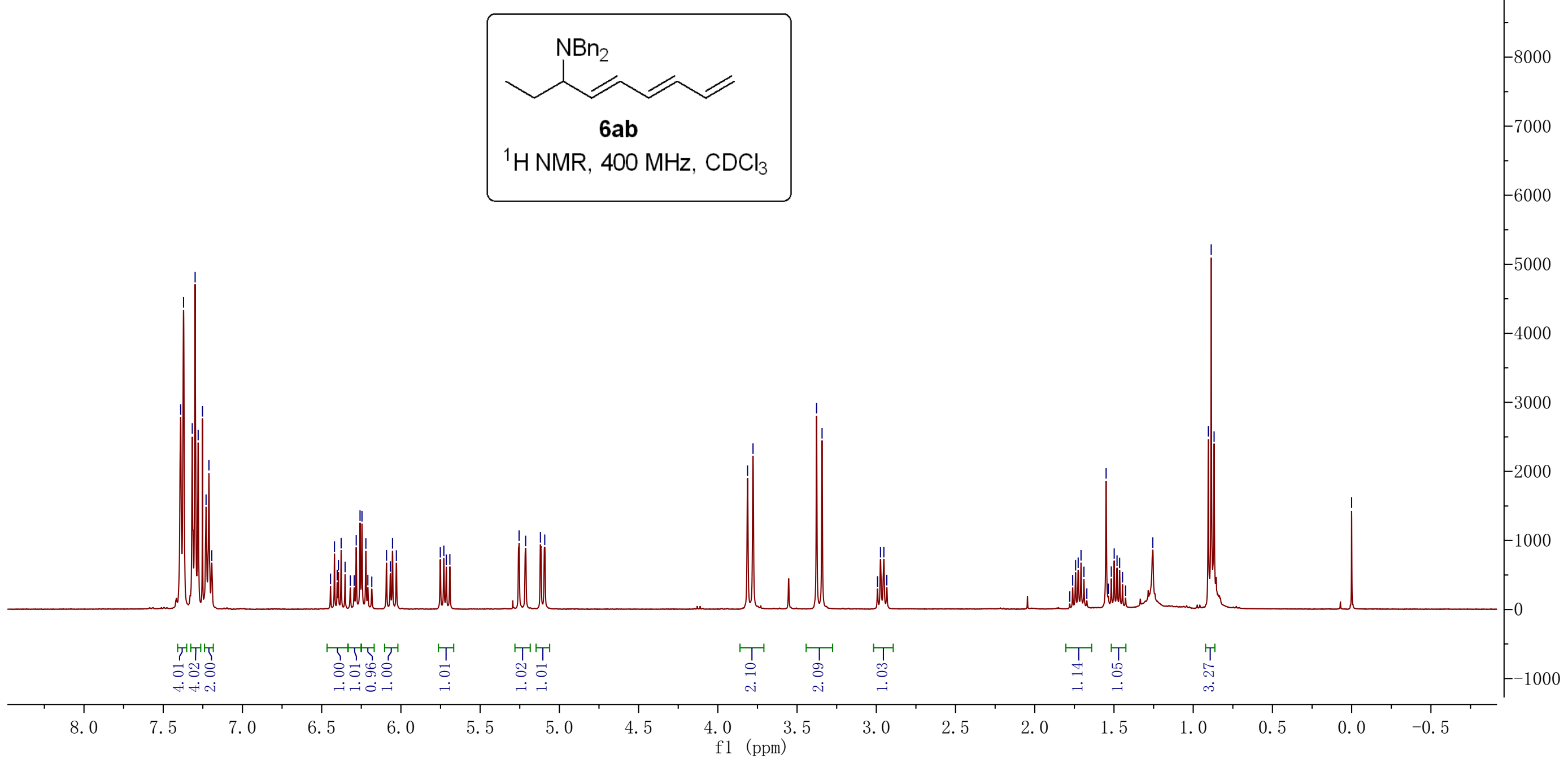




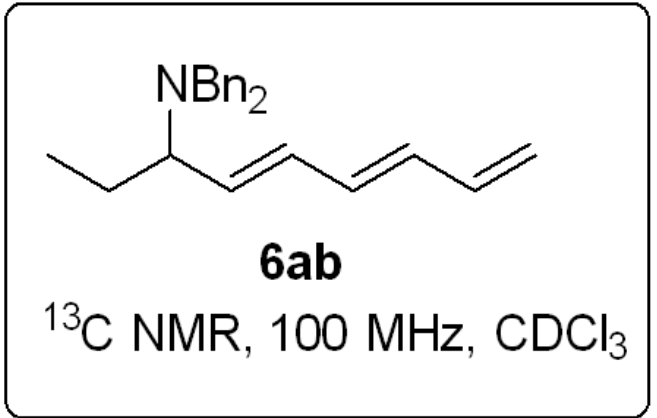




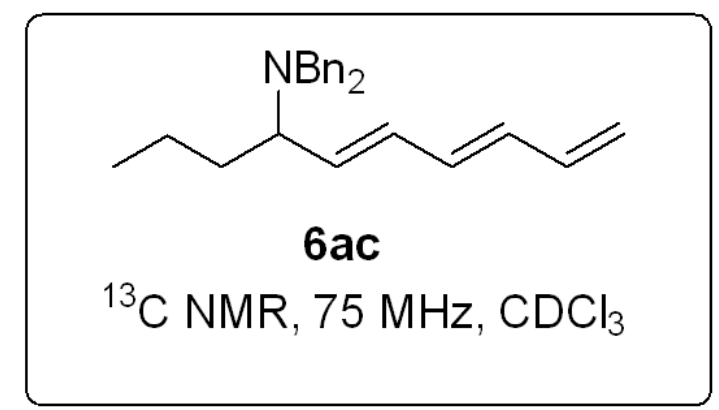

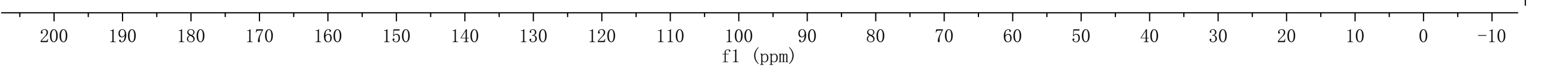



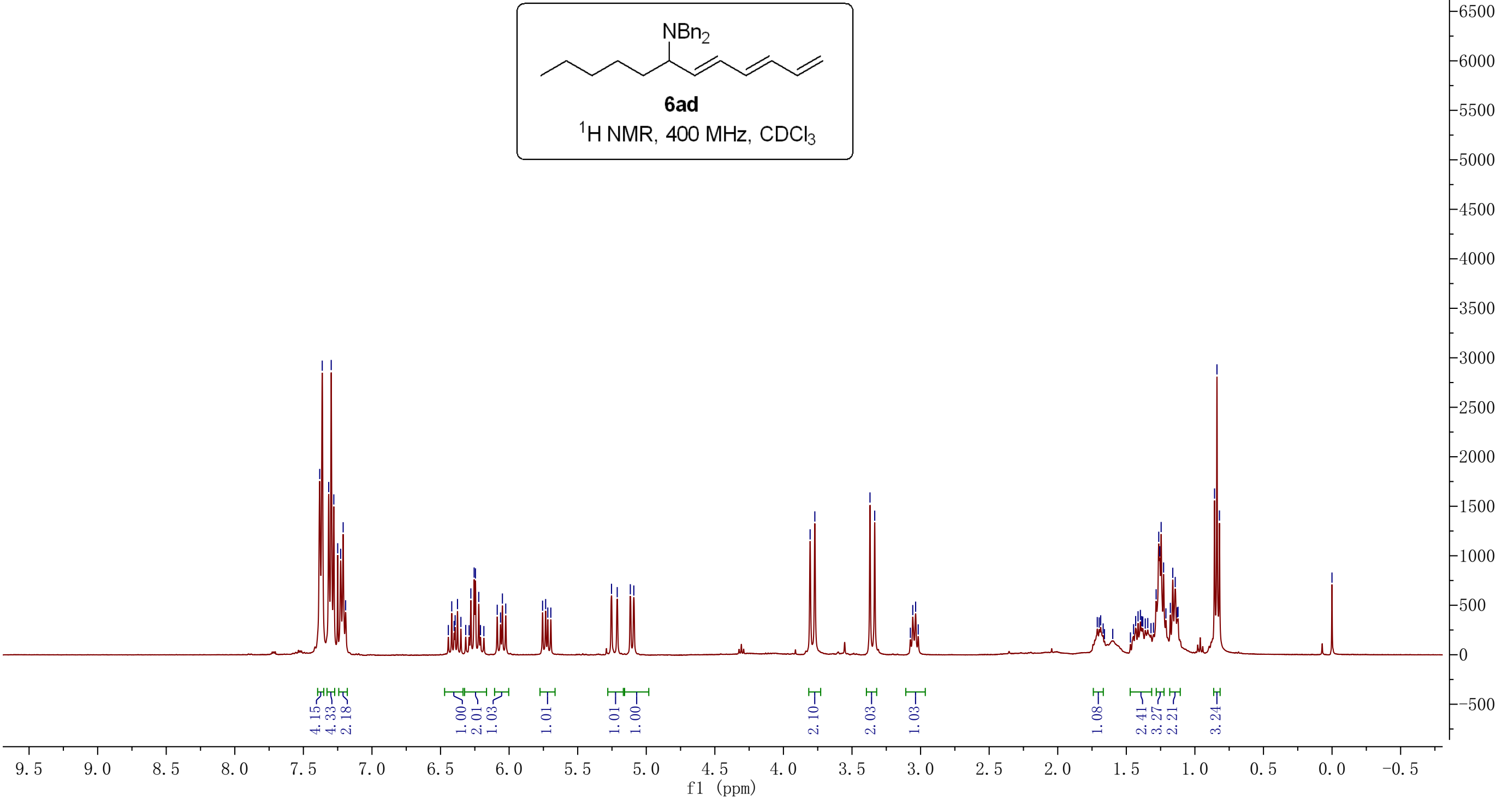
$\mathrm{NBn}_{2}$

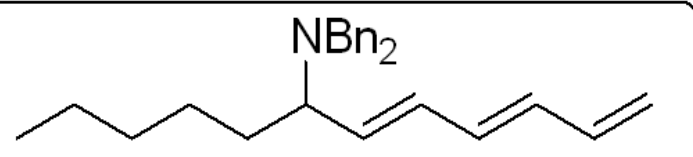

6ad

${ }^{13} \mathrm{C} \mathrm{NMR}, 100 \mathrm{MHz}, \mathrm{CDCl}_{3}$ 


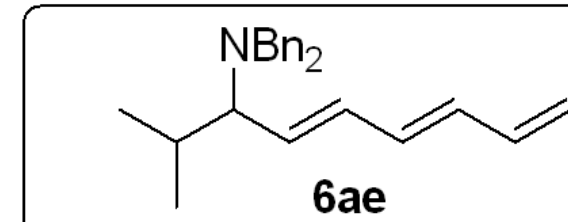

${ }^{1} \mathrm{H}$ NMR, $300 \mathrm{MHz}, \mathrm{CDCl}_{3}$

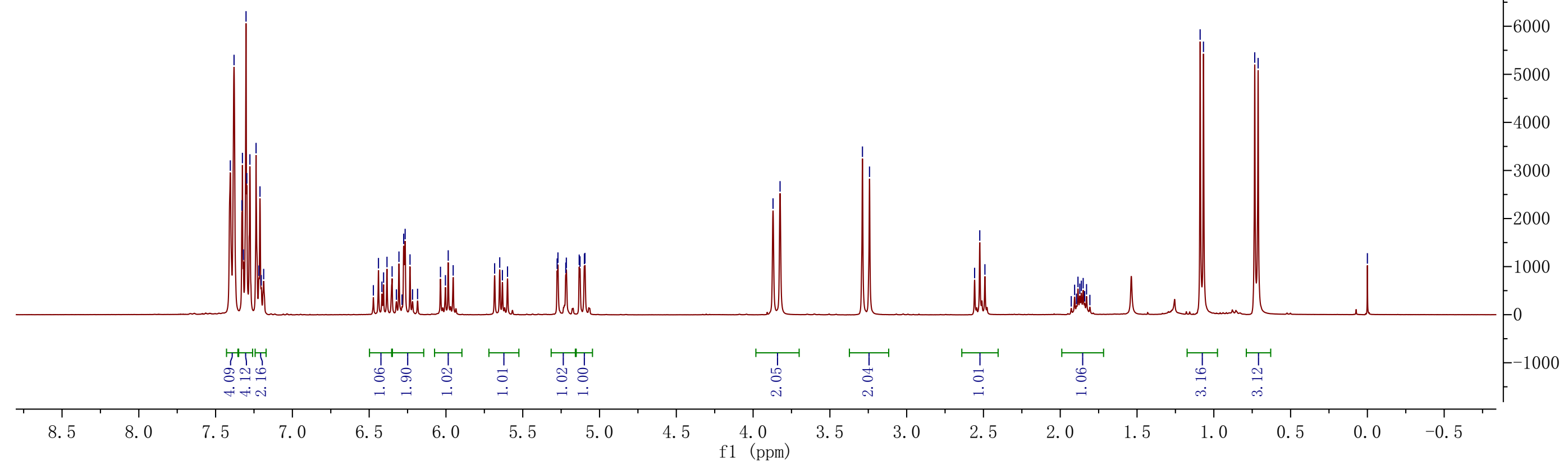



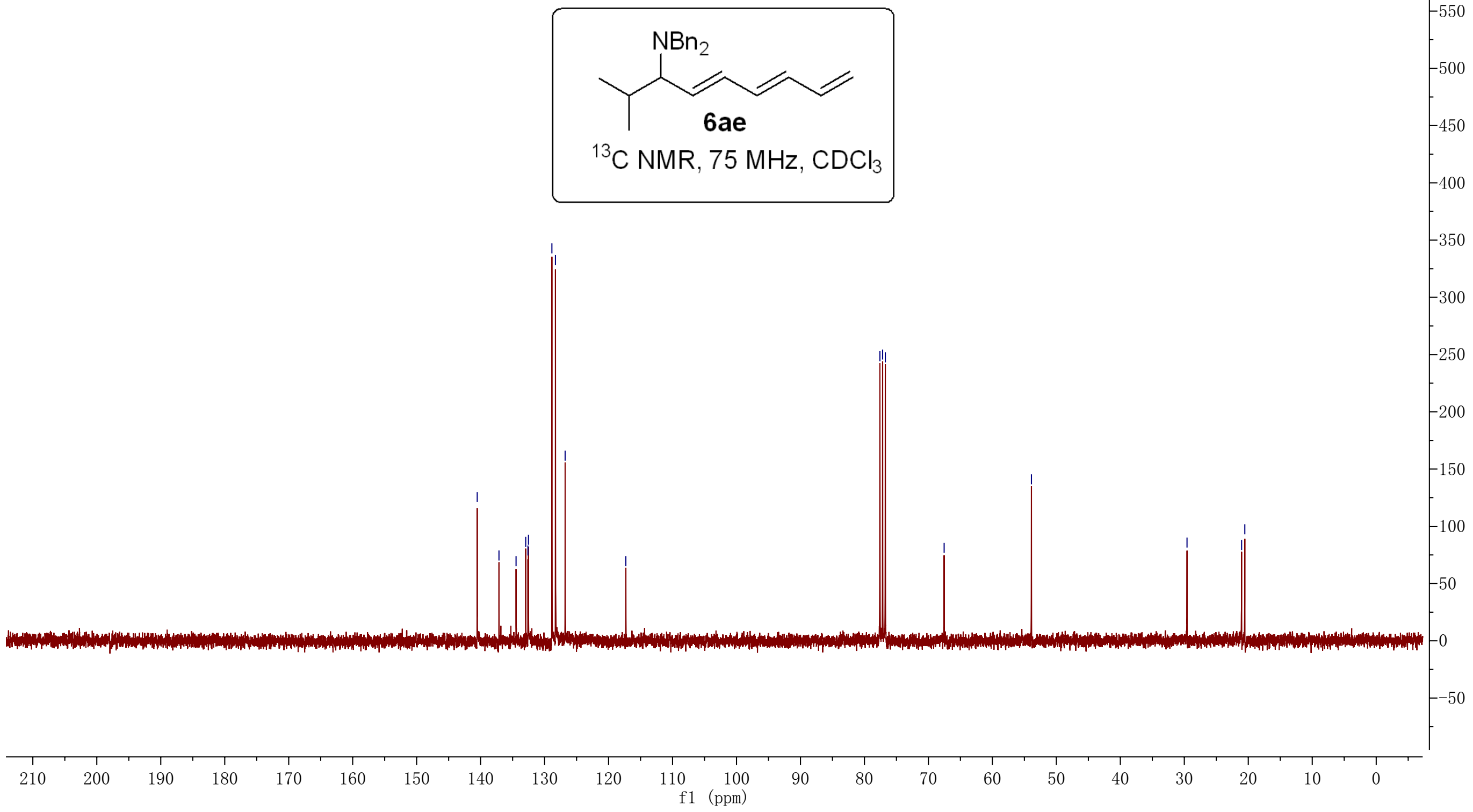


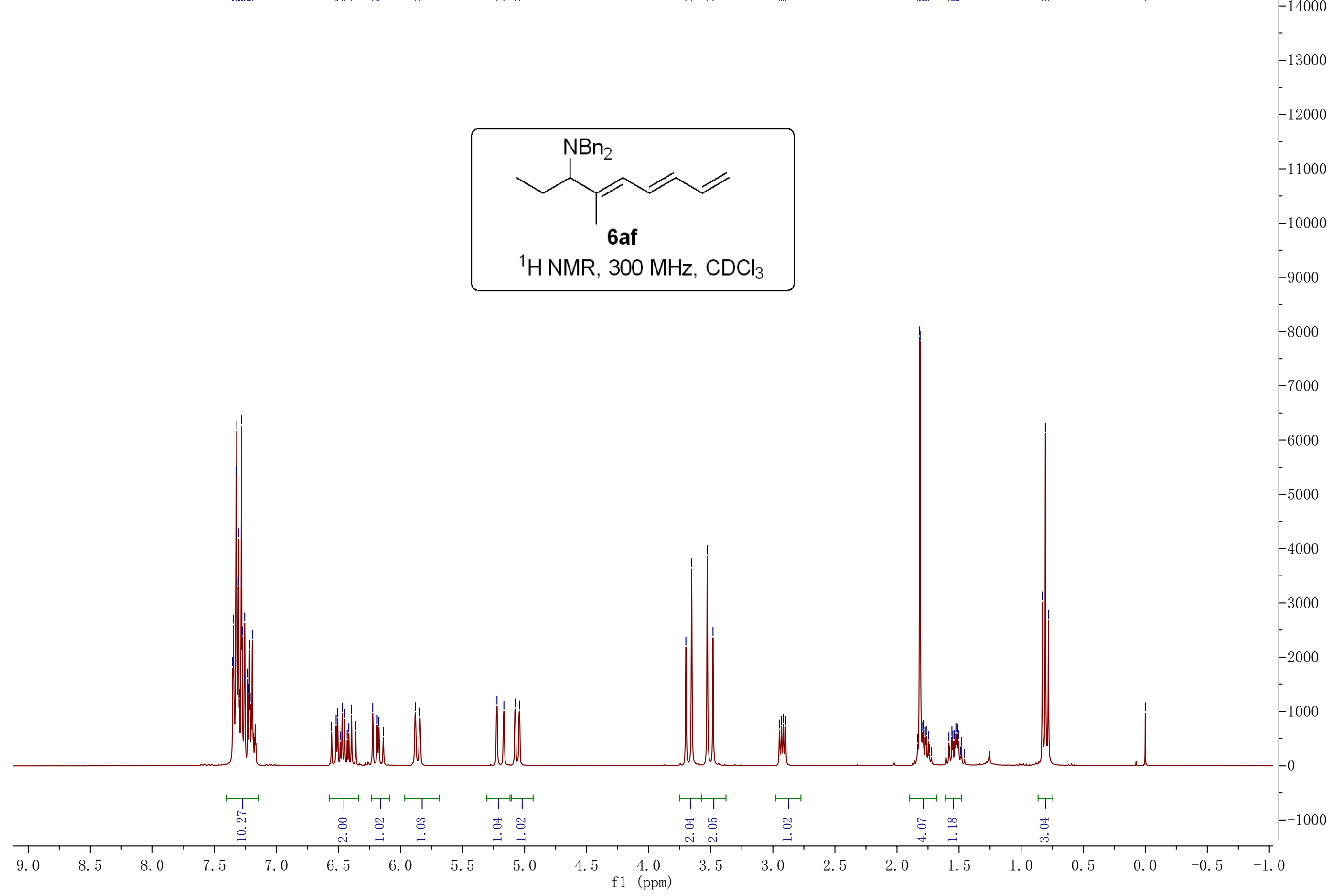



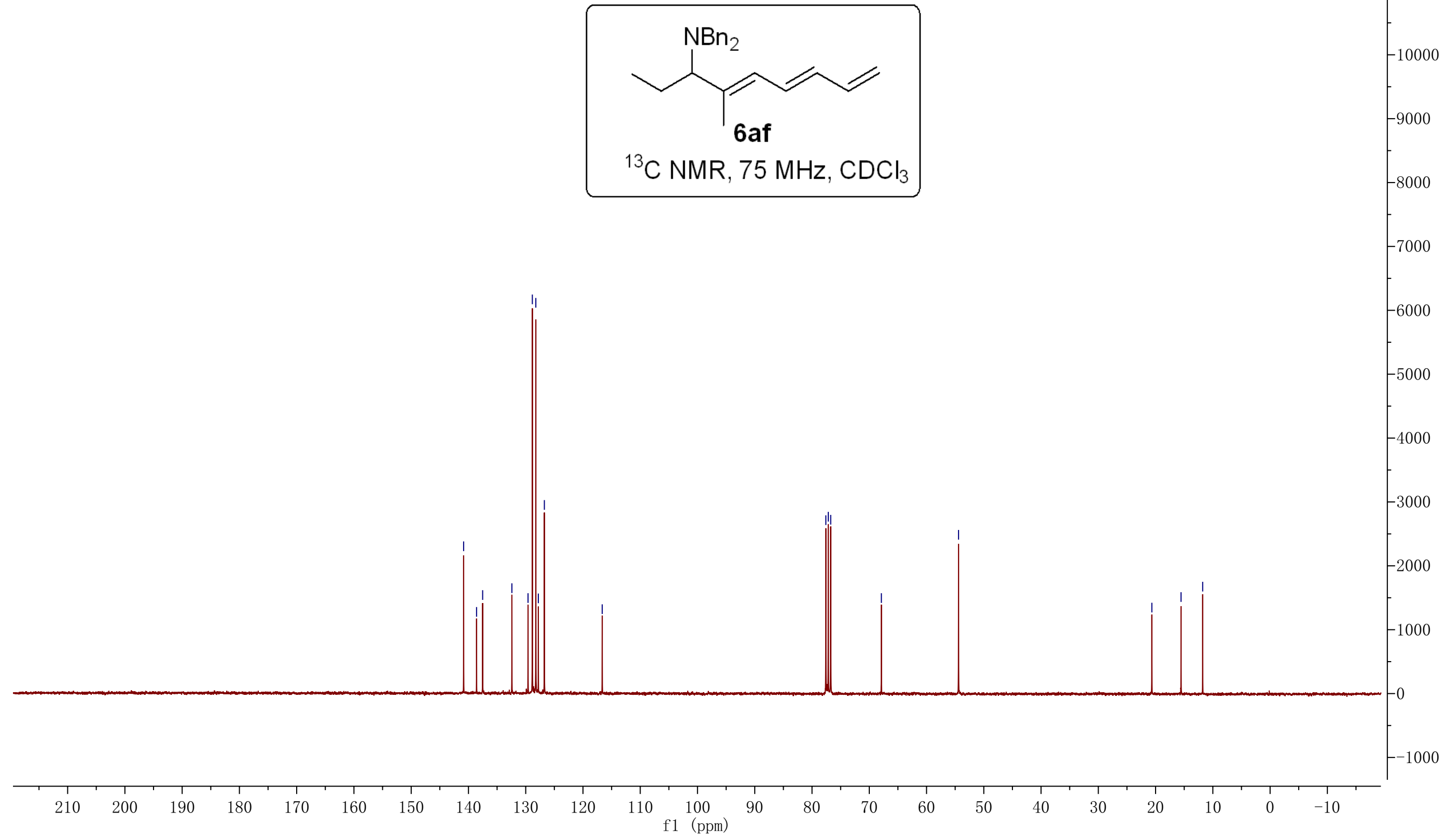

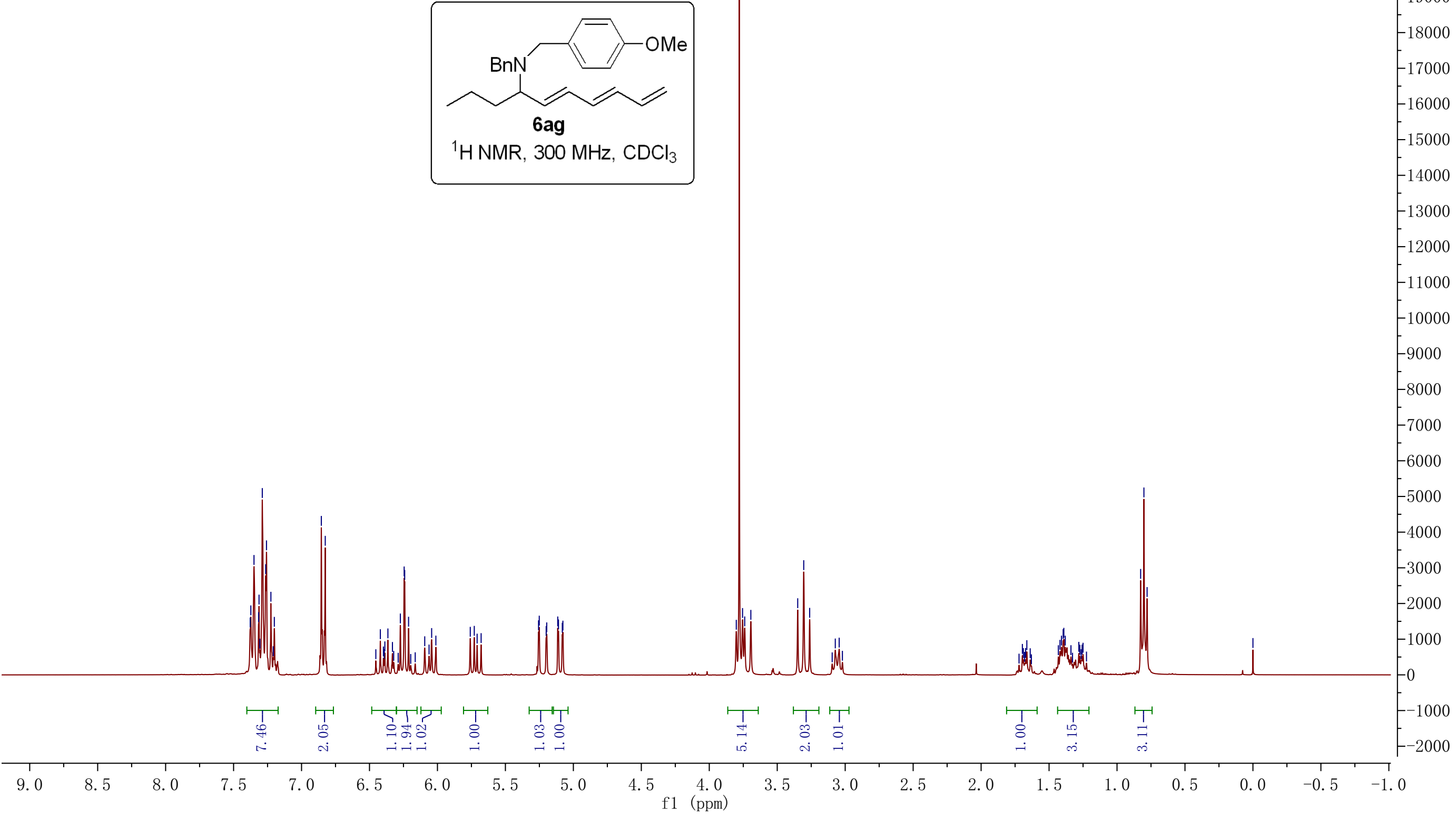

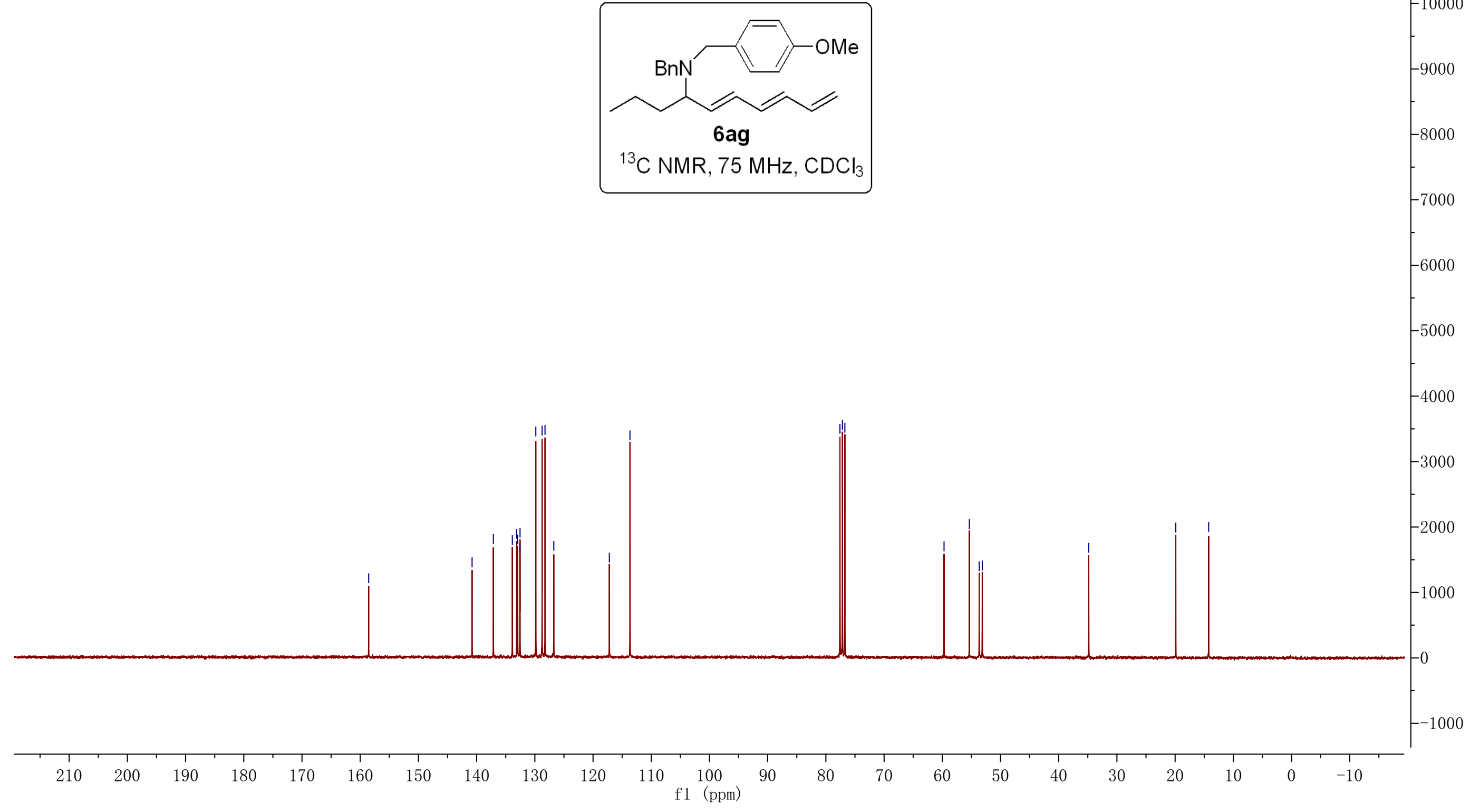

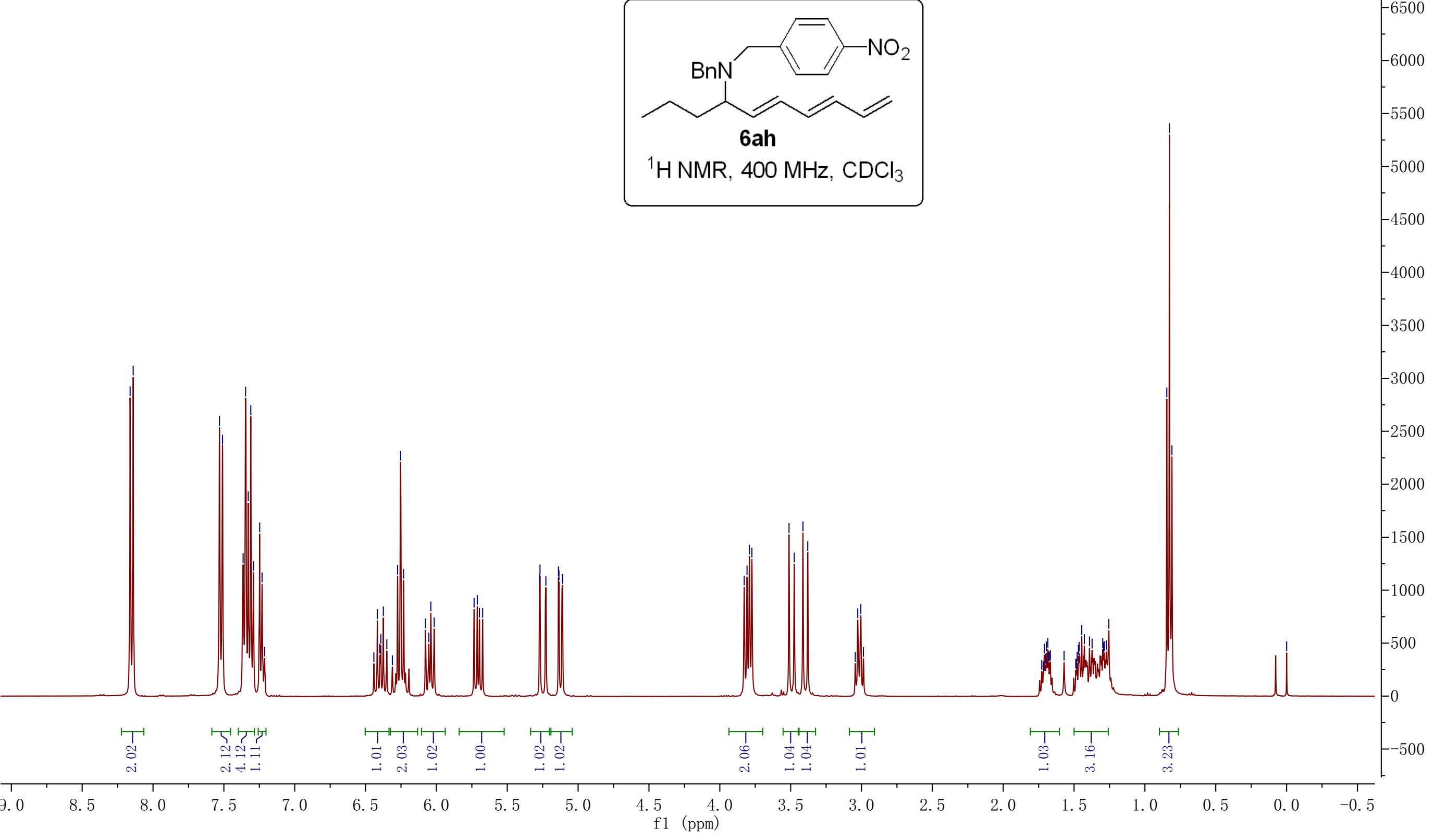


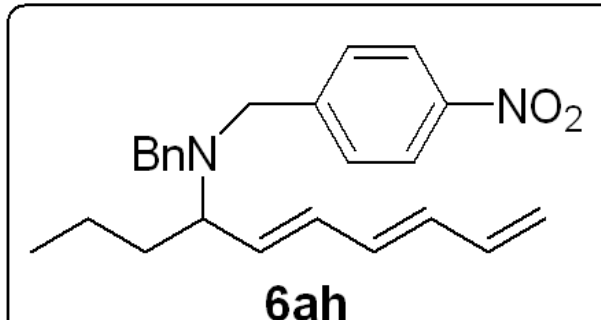

${ }^{13} \mathrm{C} \mathrm{NMR}, 100 \mathrm{MHz}, \mathrm{CDCl} /$

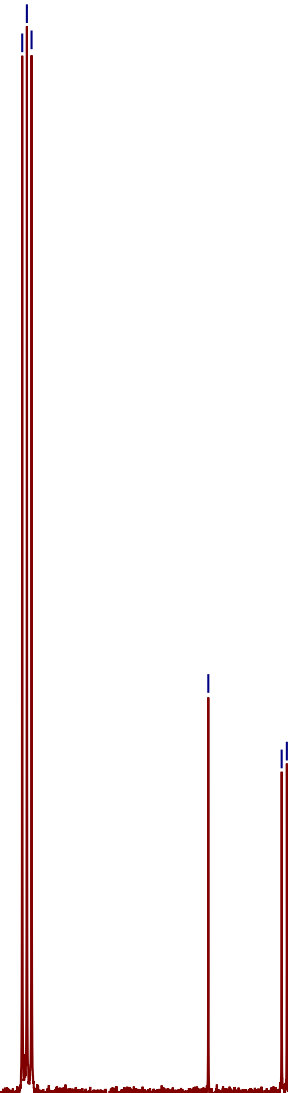



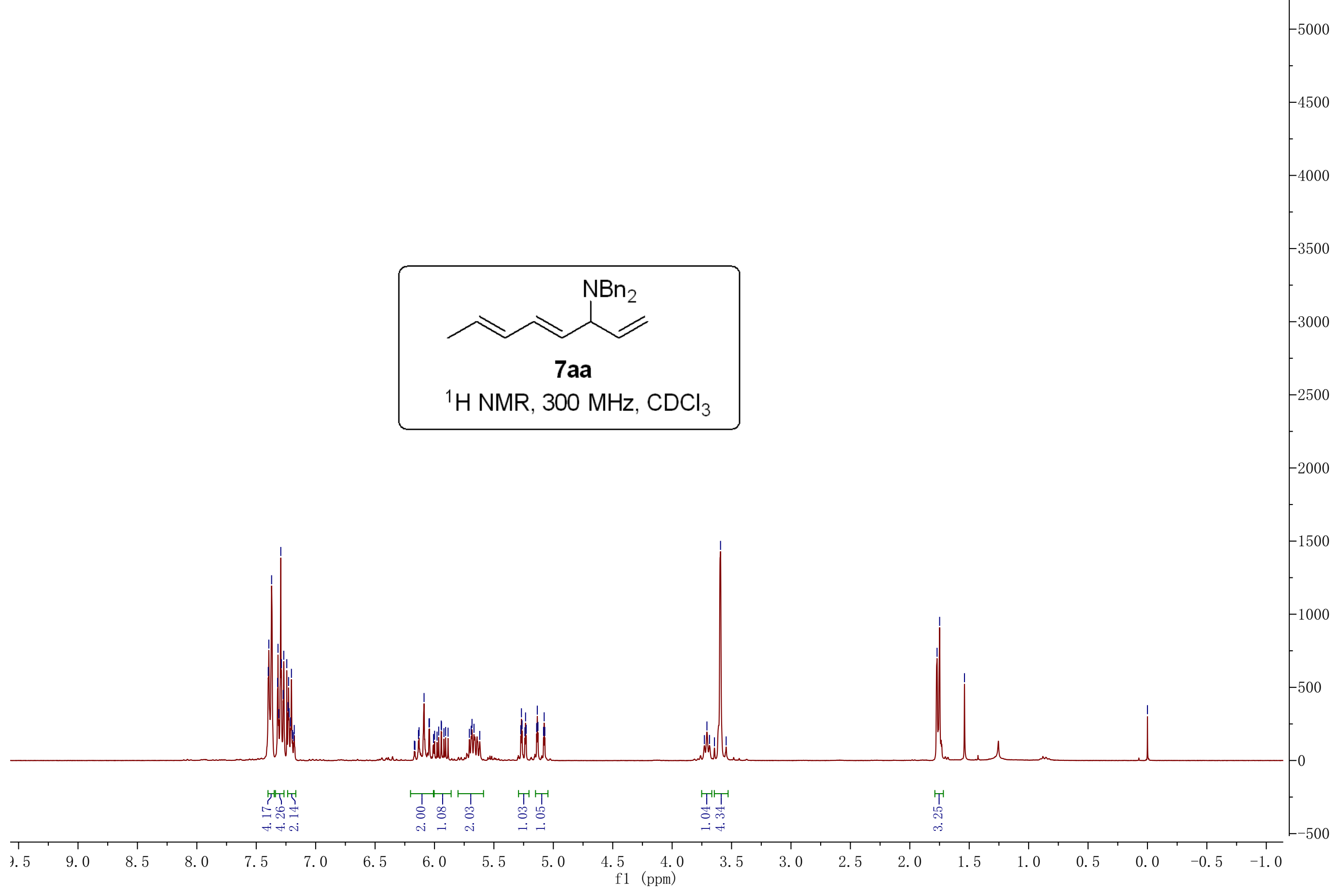


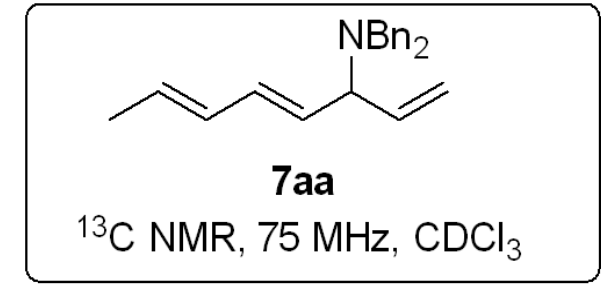



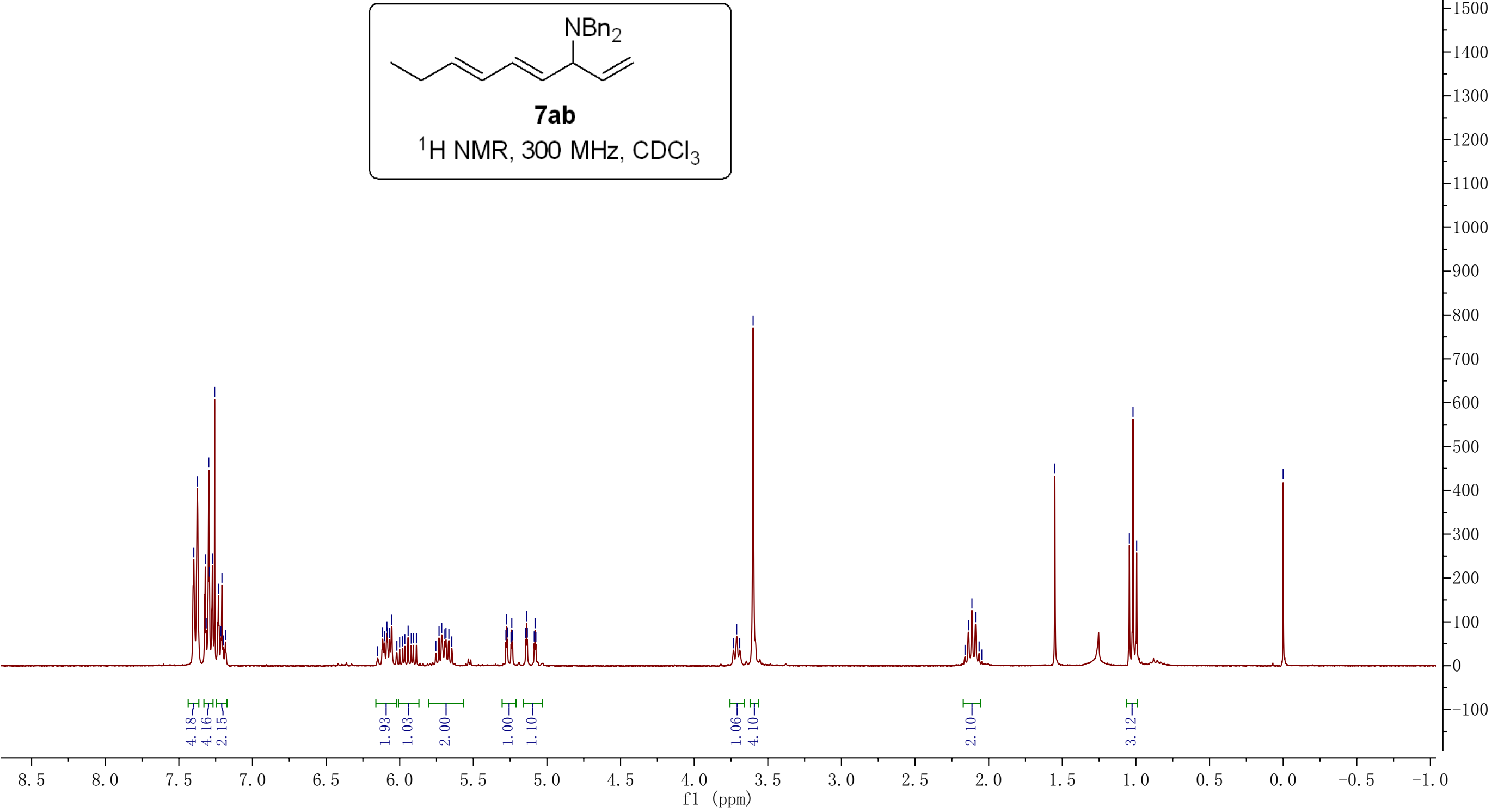


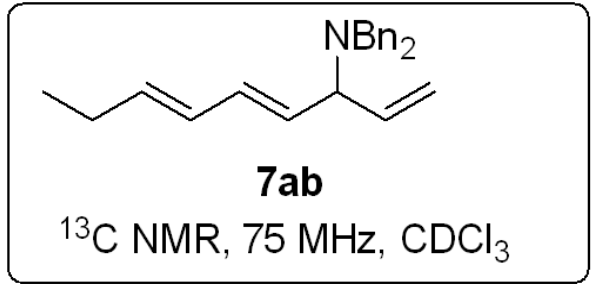

${ }^{13} \mathrm{C}$ NMR, $75 \mathrm{MHz}, \mathrm{CDCl}_{3}$ 

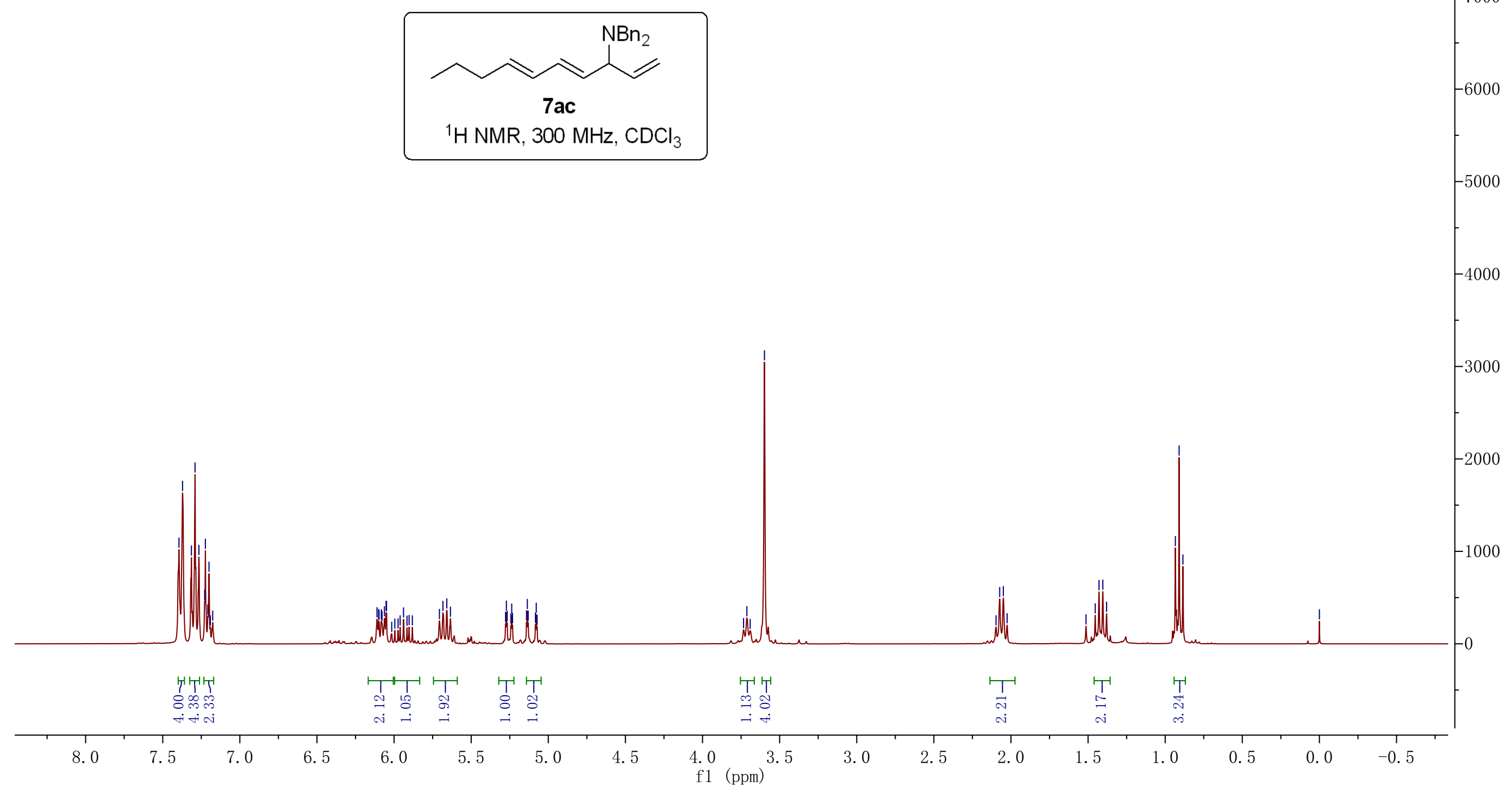


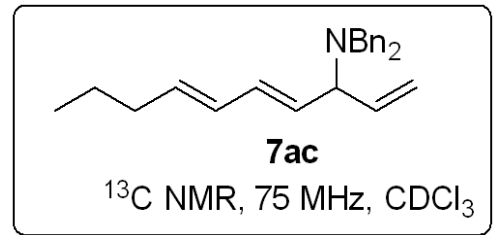



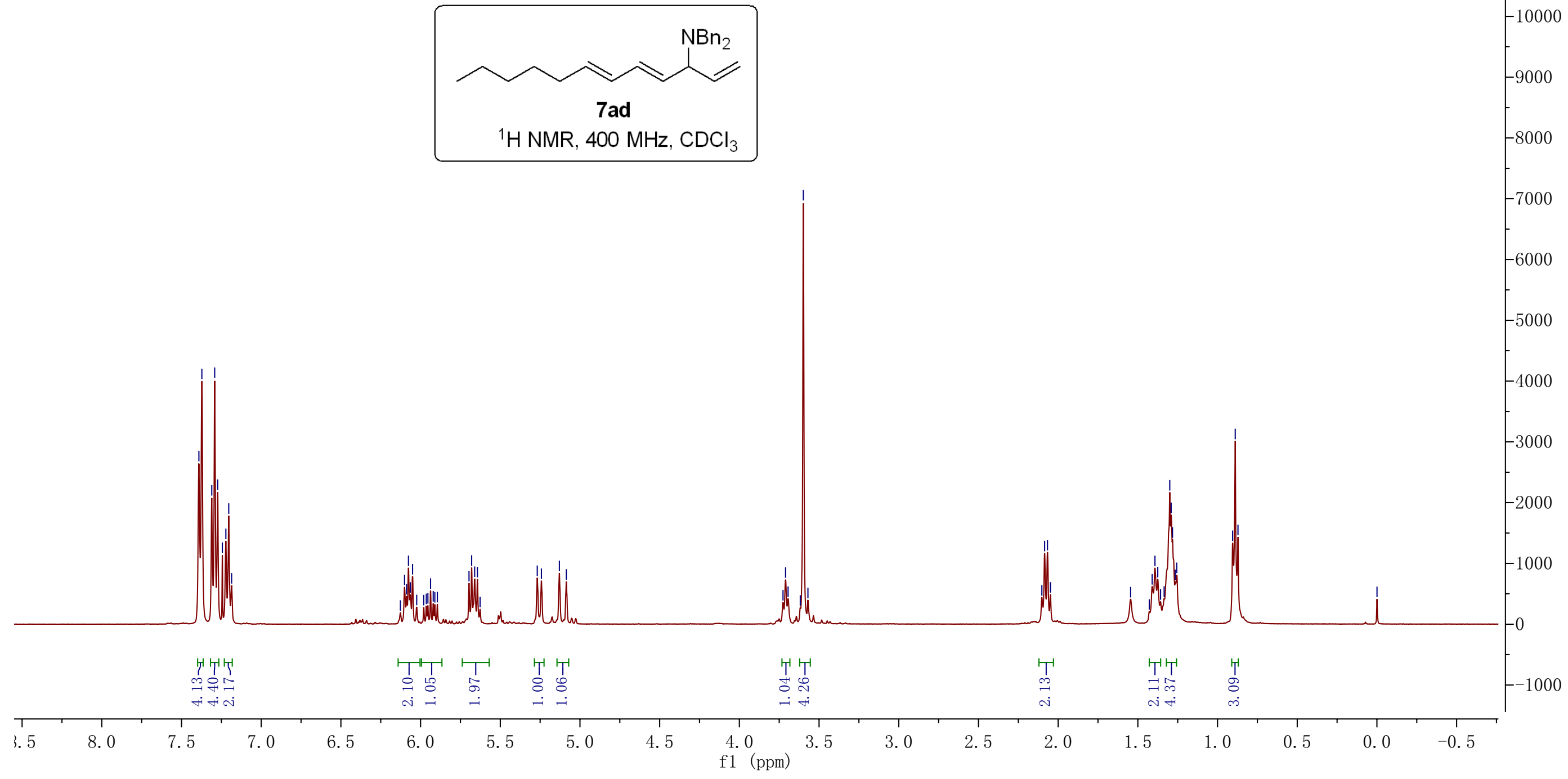

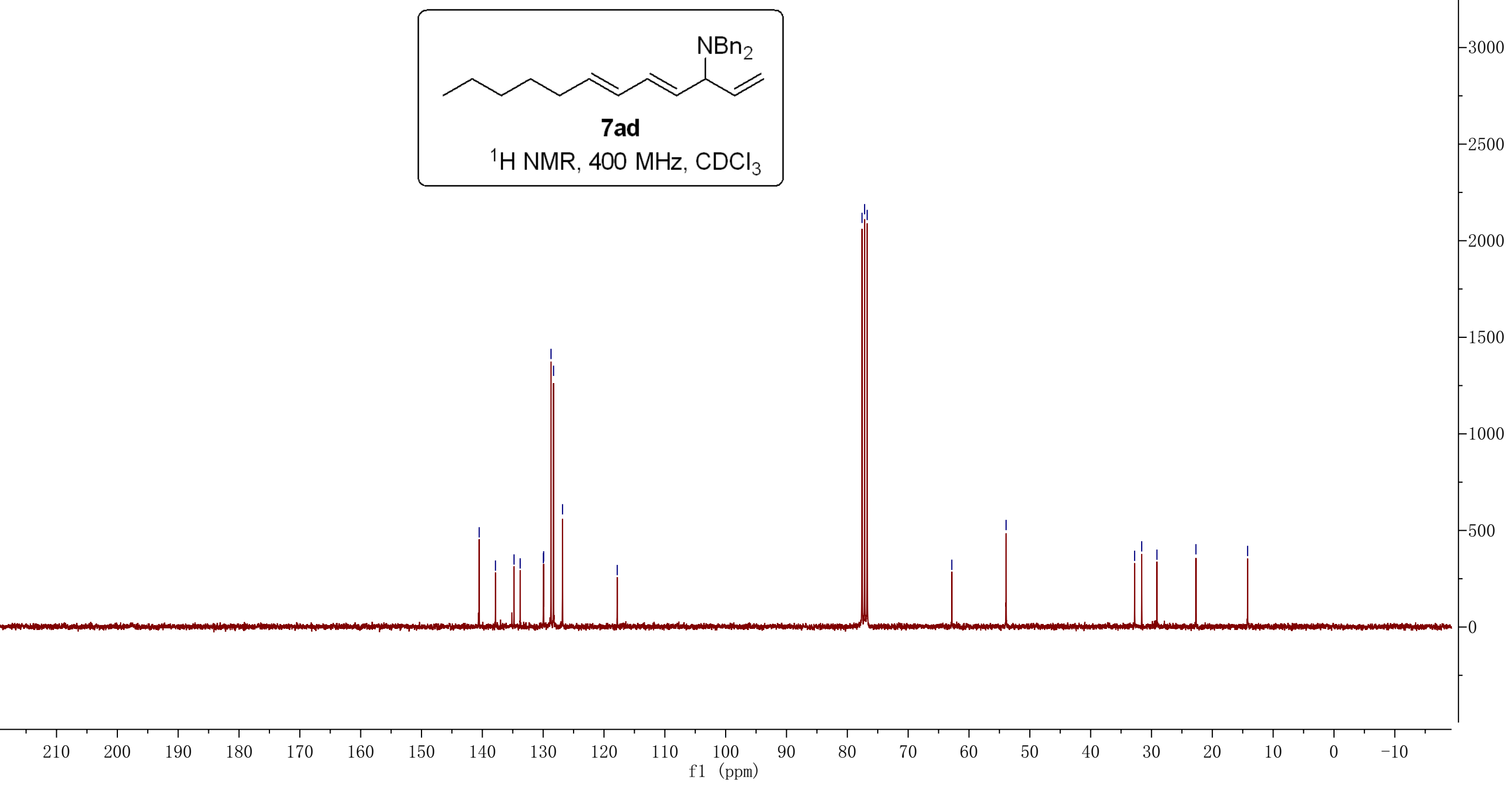

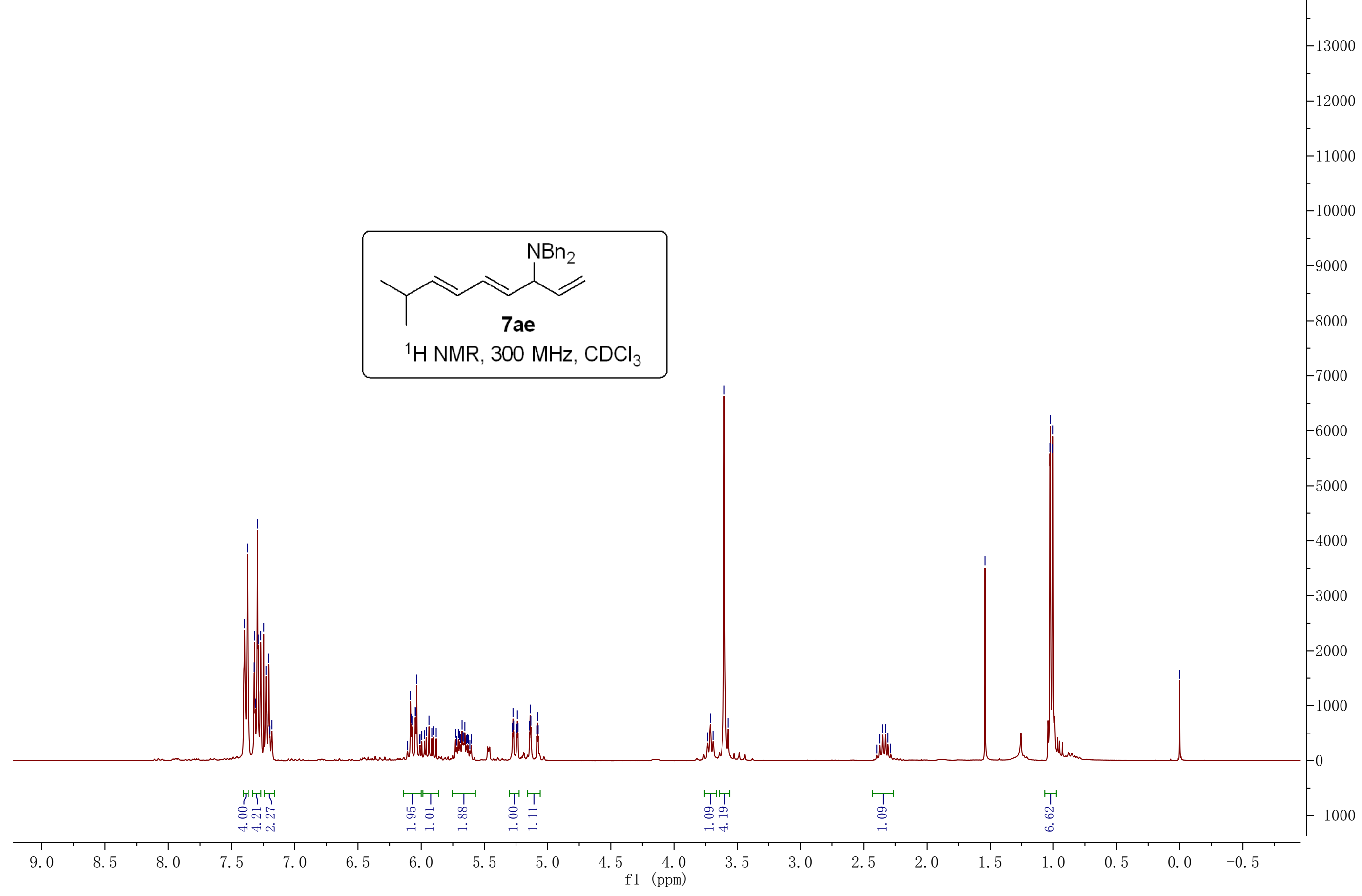


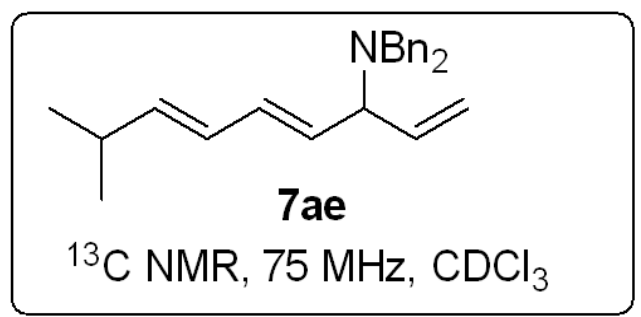




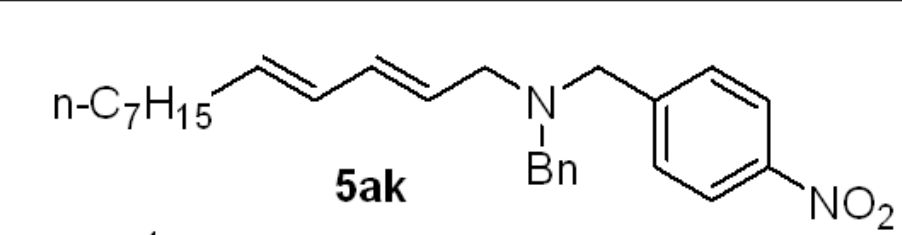

${ }^{1} \mathrm{HNMR}, 400 \mathrm{MHz}, \mathrm{CDCl}_{3}$

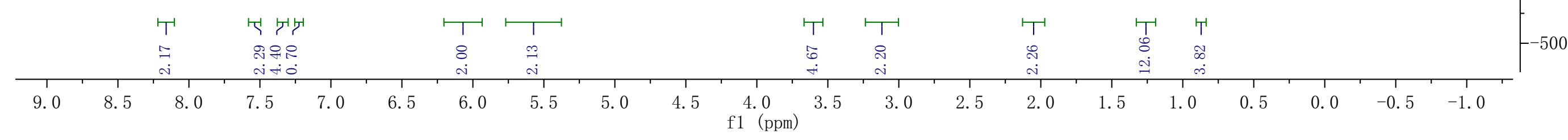




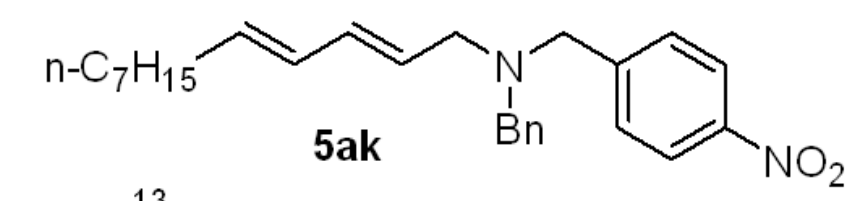

${ }^{13} \mathrm{H} \mathrm{NMR}, 100 \mathrm{MHz}, \mathrm{CDCl}_{3}$ 


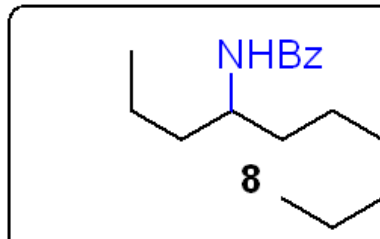

${ }^{1} \mathrm{HNMR}, 300 \mathrm{MHz}, \mathrm{CDCl}_{3}$

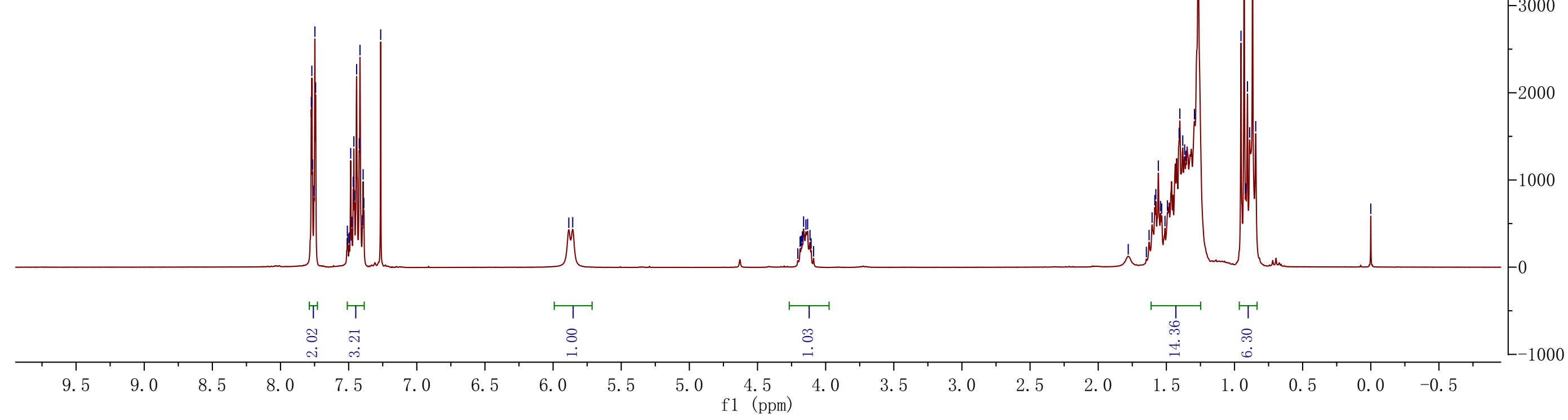


<smiles>CCCC1CCC1</smiles>

${ }^{13} \mathrm{CNMR}, 75 \mathrm{MHz}, \mathrm{CDCl}_{3}$

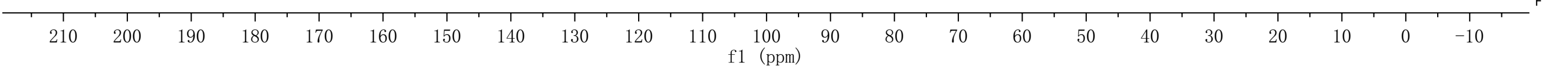




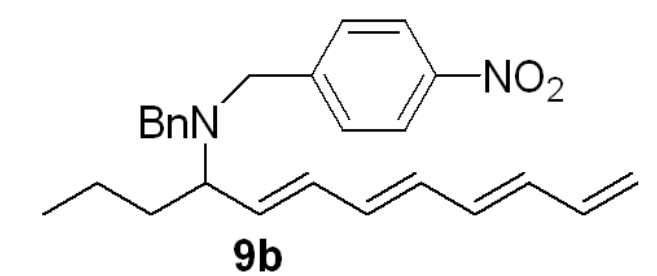

${ }^{1} \mathrm{HNMR}, 400 \mathrm{MHz}, \mathrm{CDCl}_{3}$

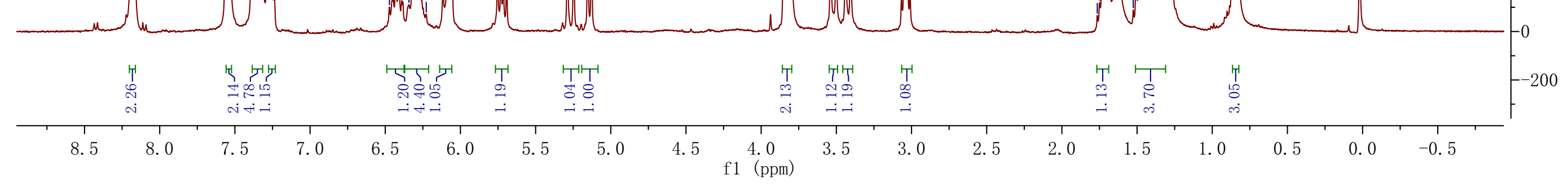



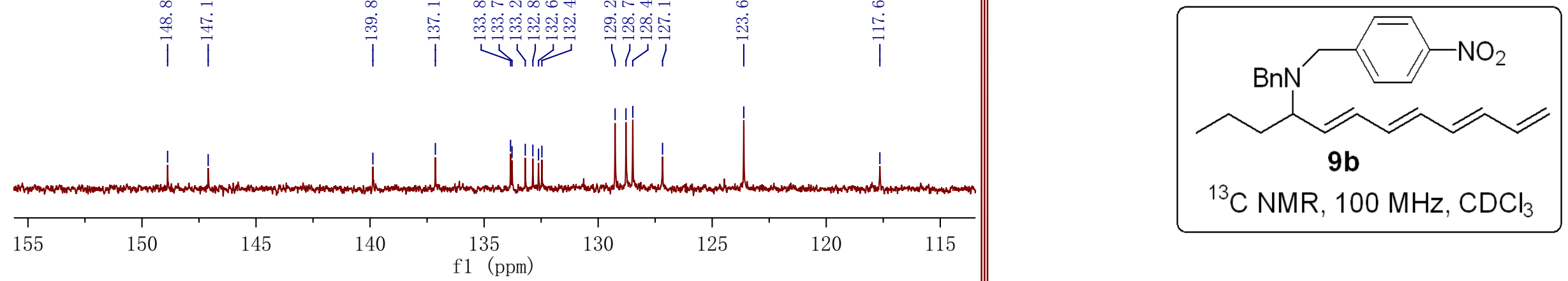

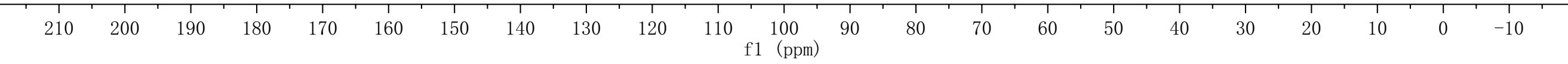


HPLC analysis of 4aa'
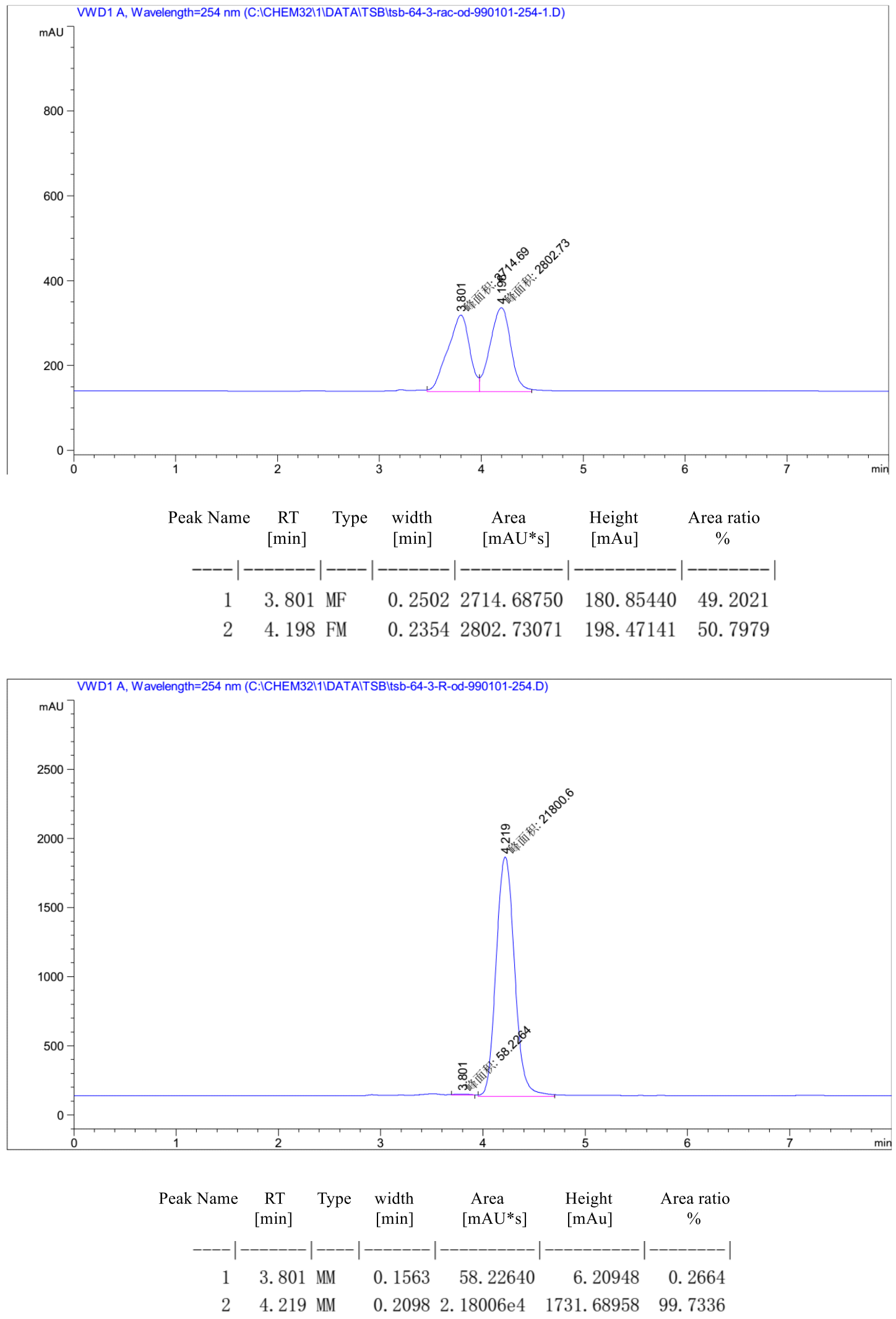


\section{HPLC analysis of $\mathbf{4} \mathbf{b a}$}
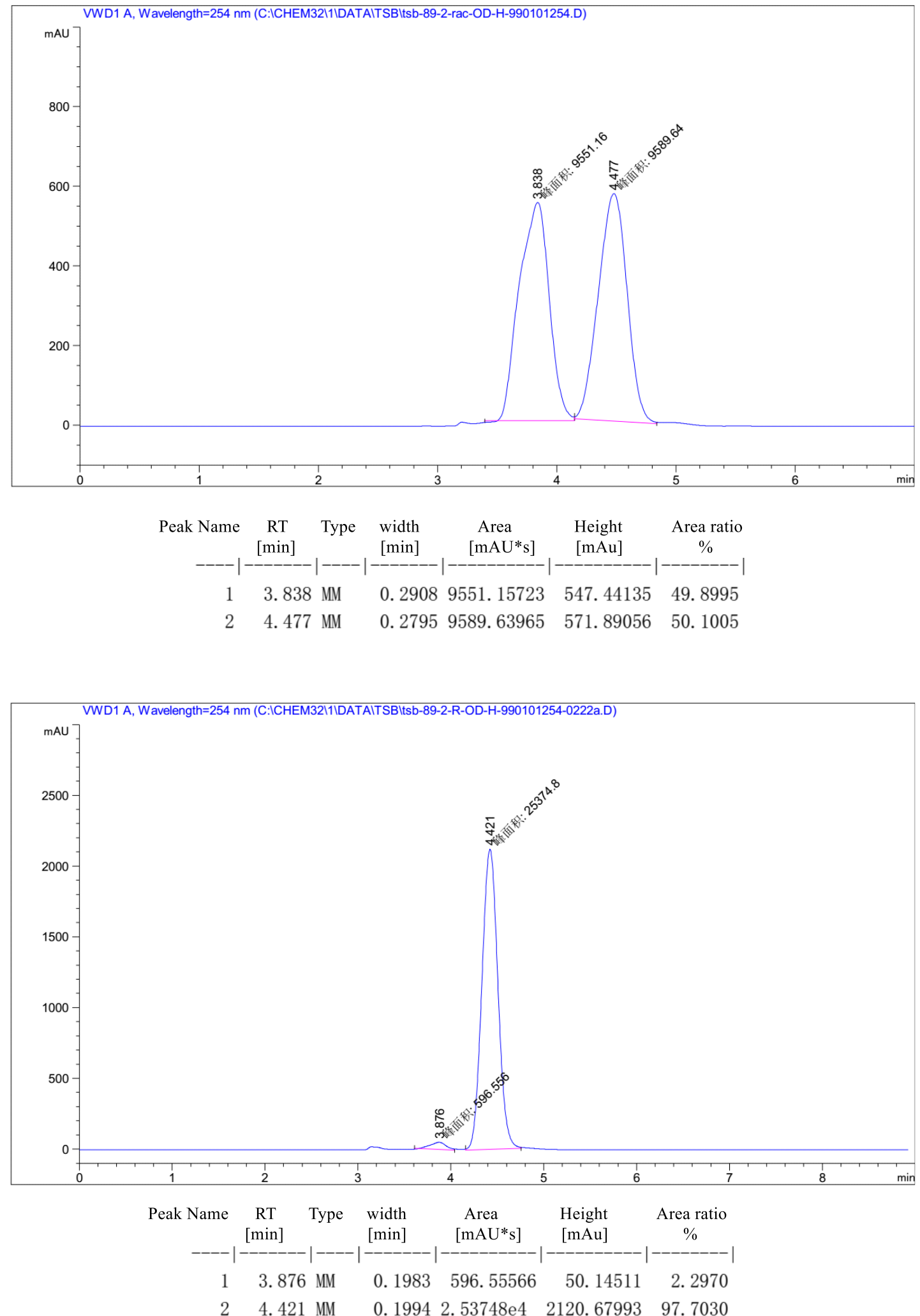


\section{HPLC analysis of 4ca'}
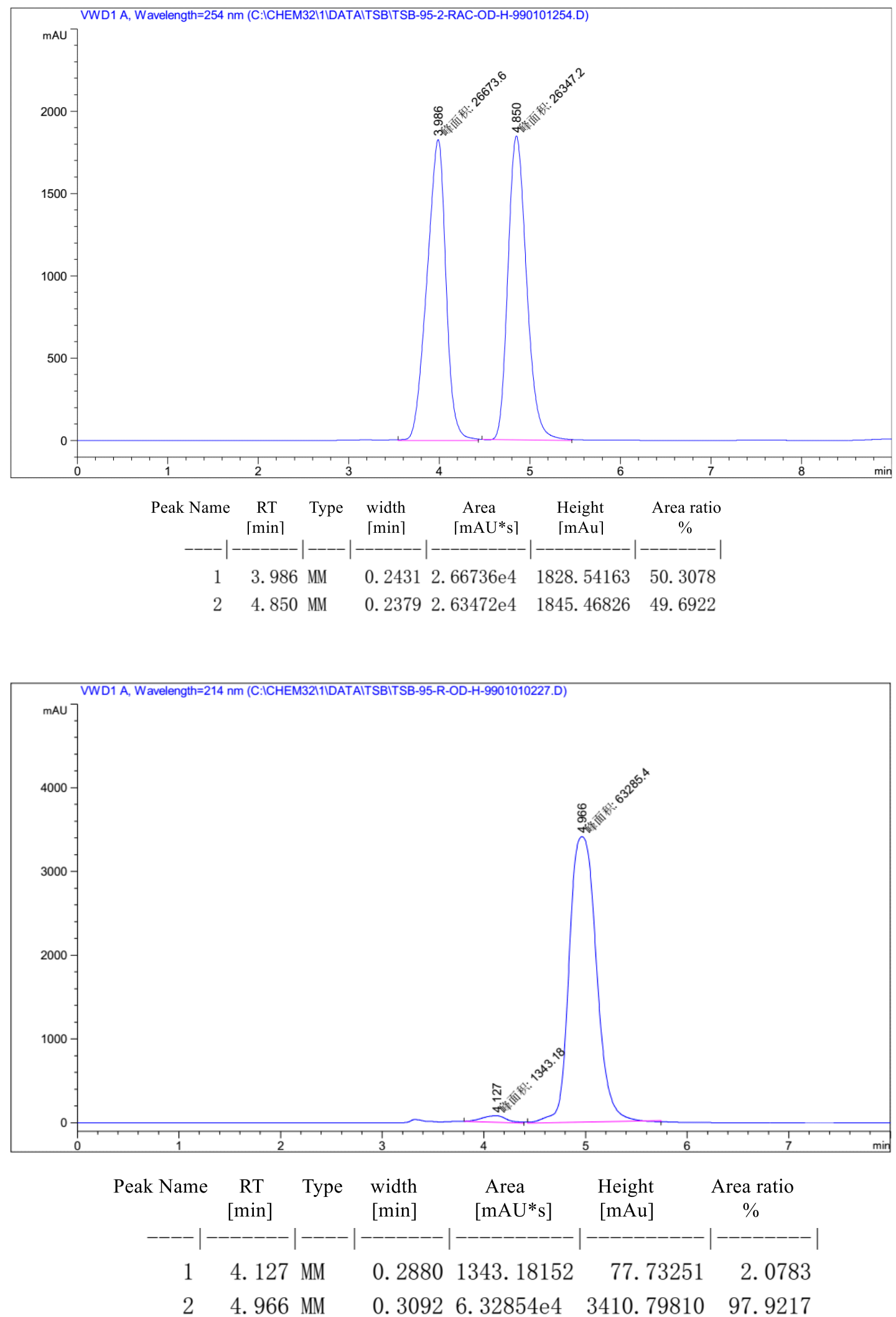


\section{HPLC analysis of $\mathbf{4 d a '}$}
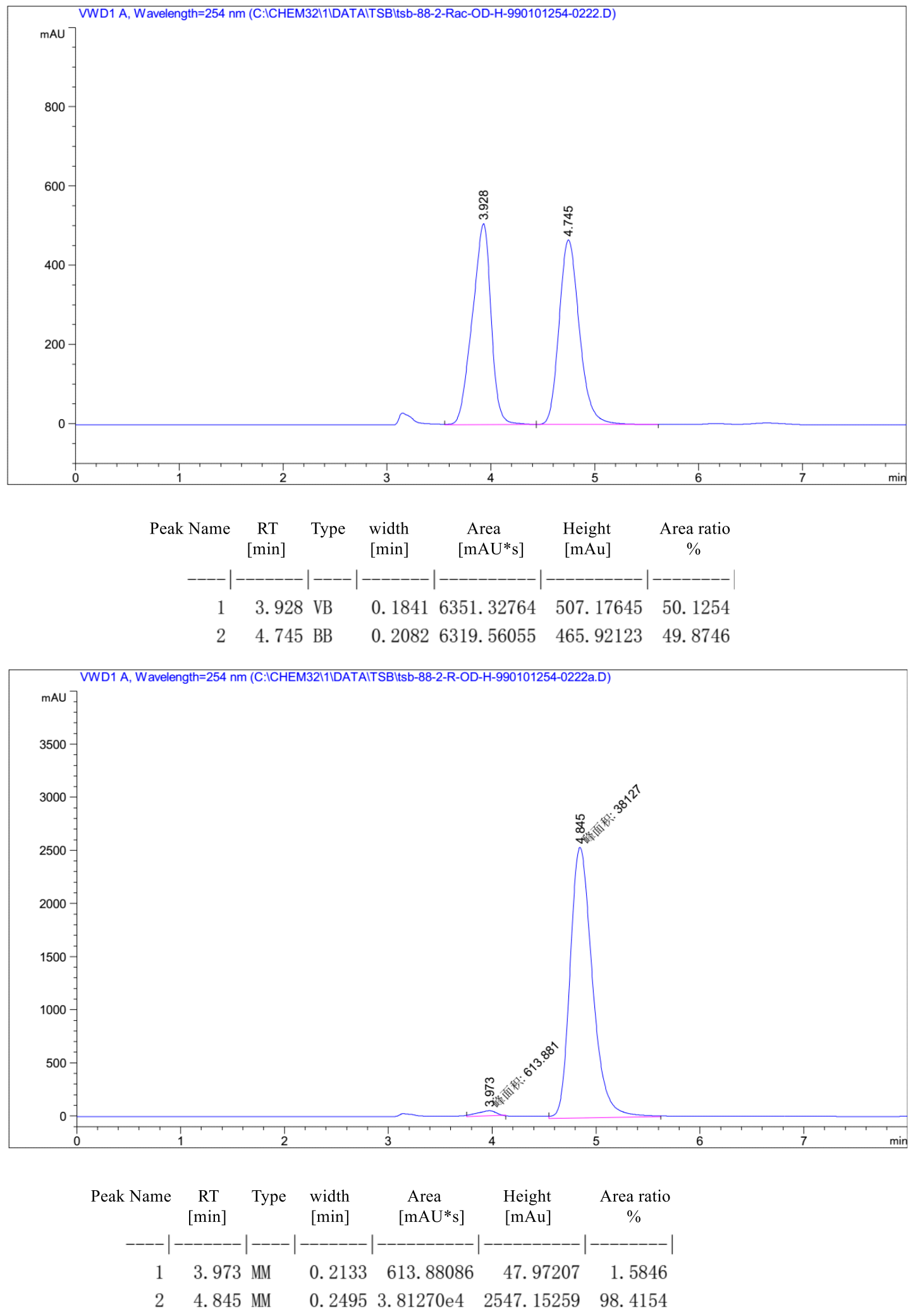


\section{HPLC analysis of 4ea'}
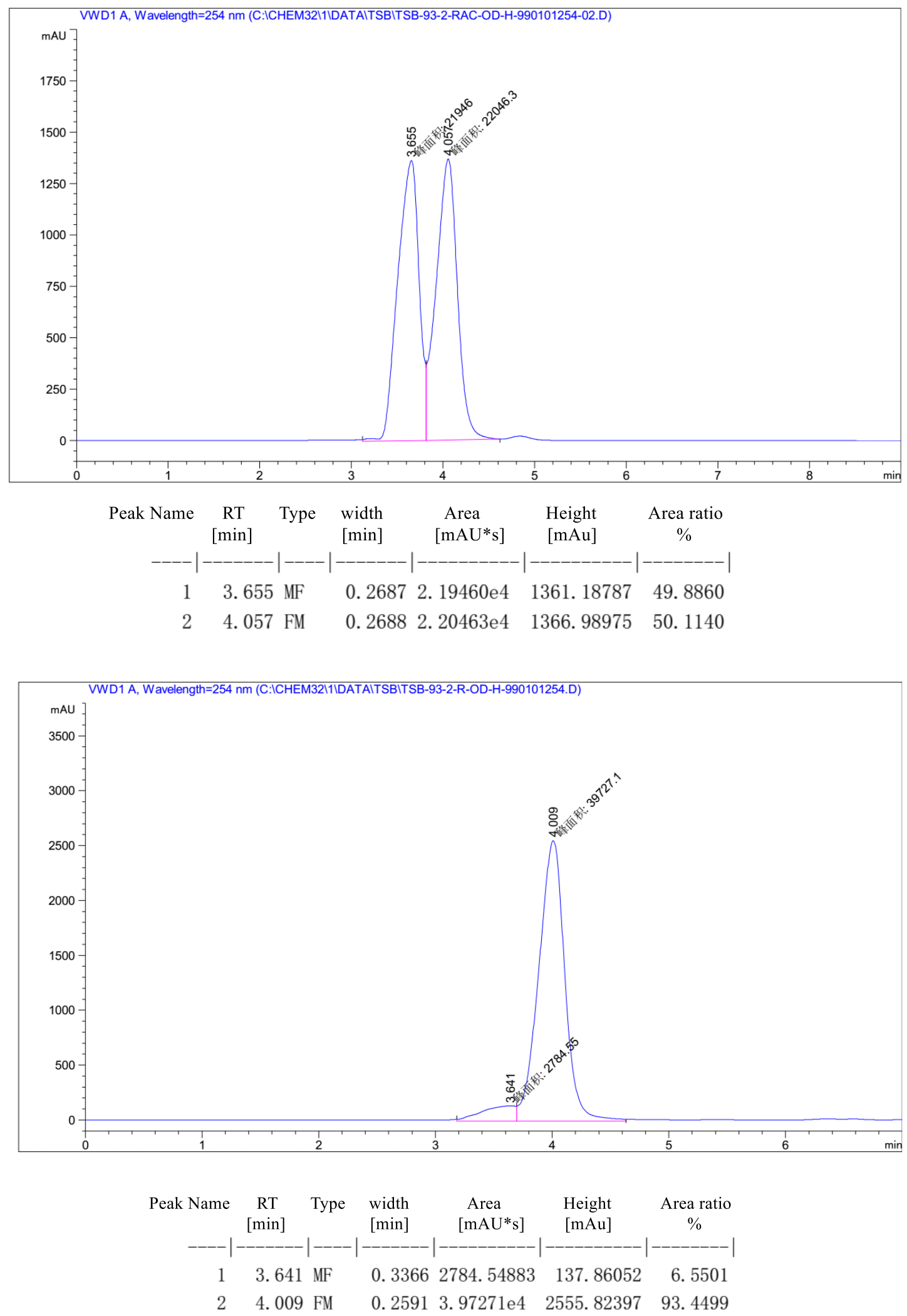


\section{HPLC analysis of $\mathbf{4 f a}$}
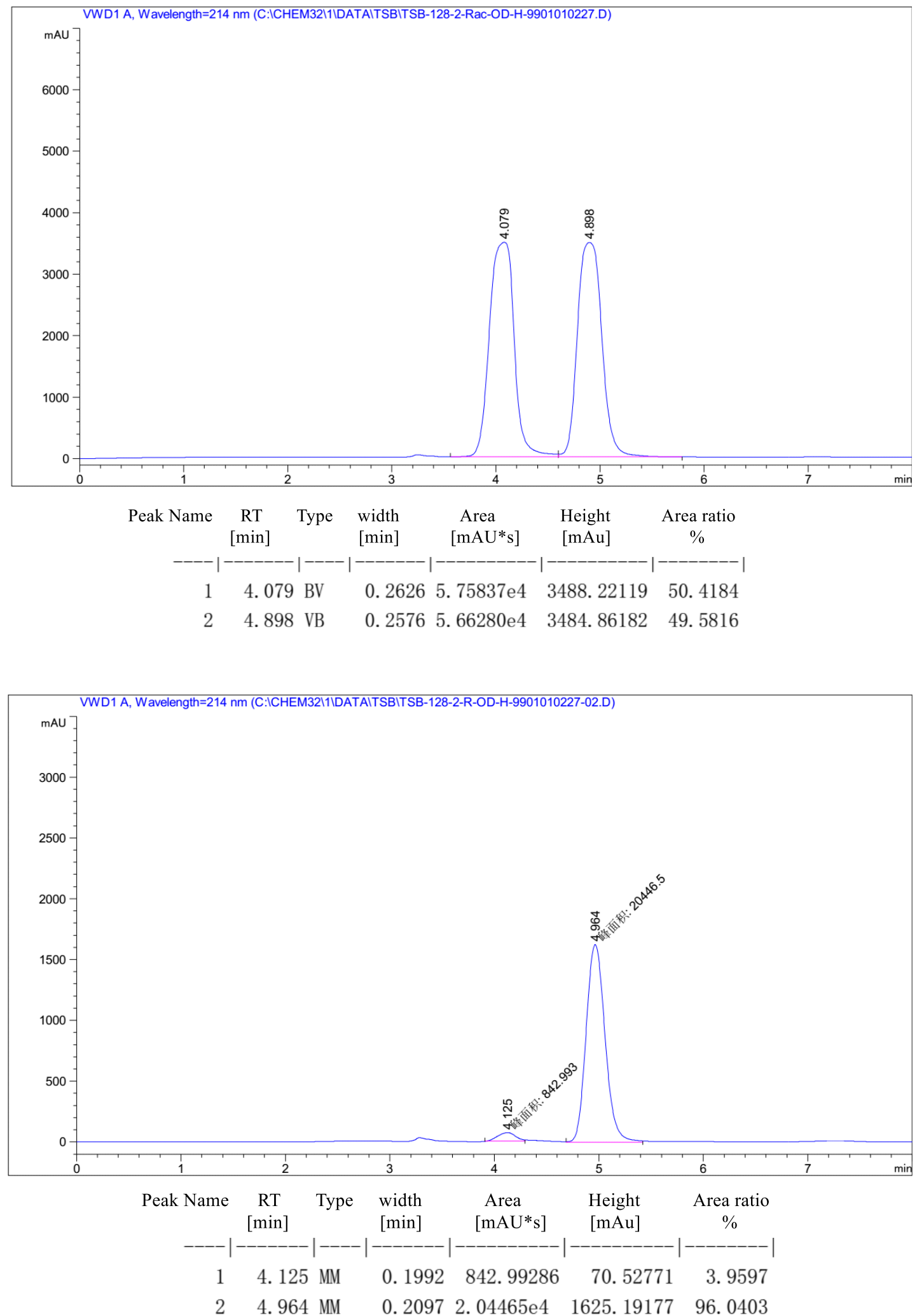


\section{HPLC analysis of 4ga'}
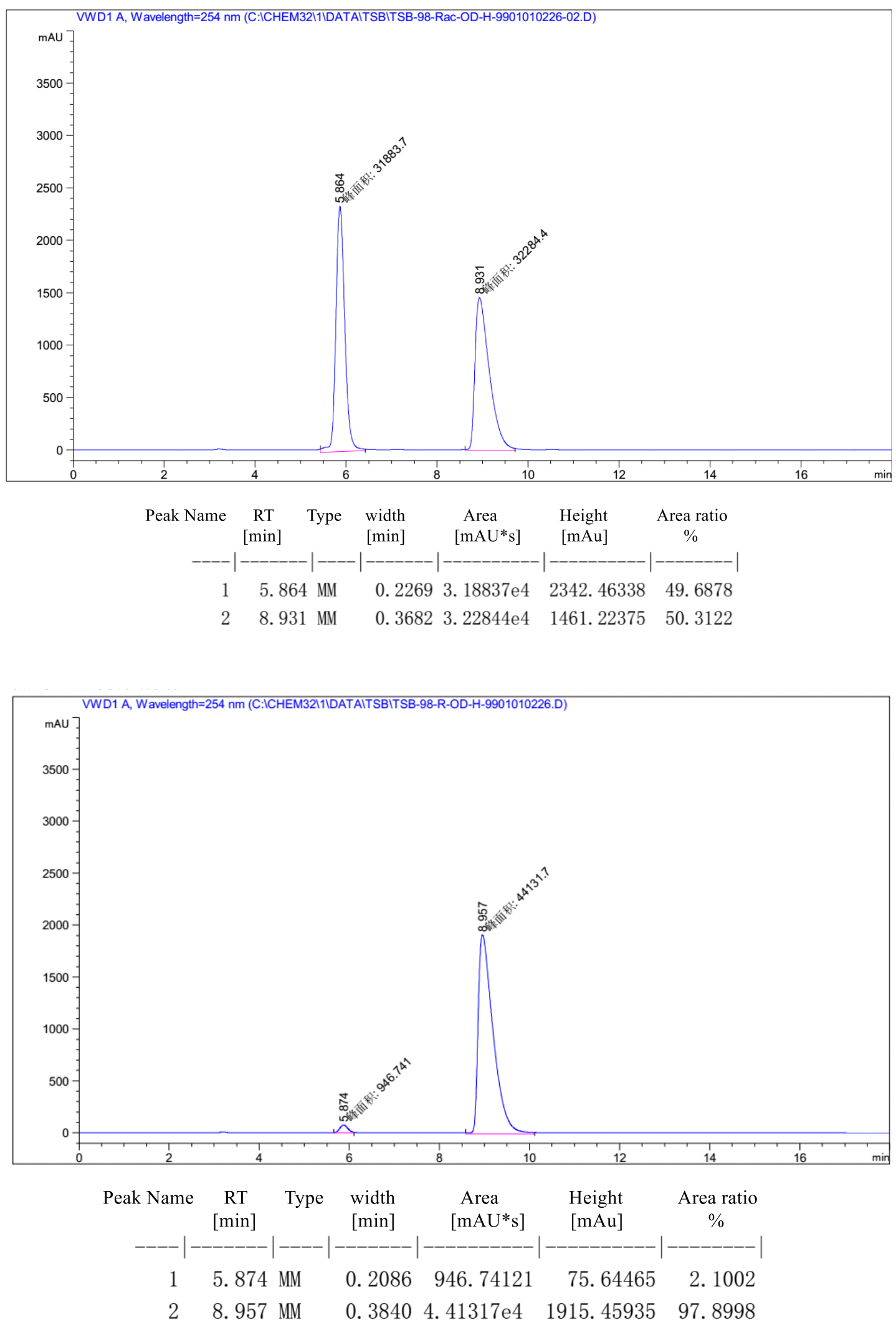